\title{
Electric-Field Induced Dissociation of Molecules in Rydberg-like Highly Vibrationally Excited Ion-Pair States
}

by

James D.D. Martin

\author{
A thesis \\ presented to the University of Waterloo \\ in fulfilment of the \\ thesis requirement for the degree of \\ Doctor of Philosophy \\ in \\ Physics
}

Waterloo, Ontario, Canada, 1998

C)James D.D. Martin 1998 
National Library

of Canada

Acquisitions and Bibliographic Services

395 Wellington Street Ottawa ON K1A ON4 Canada
Bibliothèque nationale du Canada

Acquisitions et services bibliographiques

395, rue Wellington Ottawa ON K1A ON4

Canada
The author has granted a nonexclusive licence allowing the National Library of Canada to reproduce, loan, distribute or sell copies of this thesis in microform, paper or electronic formats.

The author retains ownership of the copyright in this thesis. Neither the thesis nor substantial extracts from it may be printed or otherwise reproduced without the author's permission.
L'auteur a accordé une licence non exclusive permettant à la Bibliothèque nationale du Canada de reproduire, prêter, distribuer ou vendre des copies de cette thèse sous la forme de microfiche/film, de reproduction sur papier ou sur format électronique.

L'auteur conserve la propriété du droit d'auteur qui protège cette thèse. $\mathrm{Ni}$ la thèse ni des extraits substantiels de celle-ci ne doivent être imprimés ou autrement reproduits sans son autorisation. 
The University of Waterloo requires the signatures of all persons using or photocopying this thesis. Please sign below, and give address and date. 


\begin{abstract}
Highly vibrationally excited (high-v) ion-pair states of molecules share many of the same properties as high- $n$ Rydberg states. In high- $v$ ion-pair states, a weakly bound pair - an anion and cation - play the roles of electron and ion-core. High- $v$ ion-pair states have an infinite number of vibrational levels below their dissociation threshold, and these follow a Rydberg-like formula. It is demonstrated that high- $v$ ion-pair states can be dissociated by the application of weak pulsed electric fields, in a similar manner to the electric-field induced ionization of high-n Rydberg states. It has been possible to exploit this effect - using a technique similar to pulsed-field ionization zero-kinetic-energy photoelectron spectroscopy (PFI-ZEKE) and mass-analyzed threshold ionization spectroscopy (MATI) - to determine field-free energetic thresholds for ion-pair formation. The thresholds for three processes were studied using this technique: $\mathrm{HCl}+h \nu \rightarrow \mathrm{H}^{+}+\mathrm{Cl}^{-}, \mathrm{O}_{2}+h \nu \rightarrow$ $\mathrm{O}^{+}+\mathrm{O}^{-}$, and $\mathrm{HF}+h \nu \rightarrow \mathrm{H}^{+}+\mathrm{F}^{-}$. The accurately determined field-free ion-pair formation threshold of $\mathrm{HCl}$, together with the known values of the ionization potential of $\mathrm{H}$ and the electron affinity of $\mathrm{Cl}$, allowed the dissociation energy of $\mathrm{HCl}$ to be determined to within $\pm 1 \mathrm{~cm}^{-1}$ - a significant improvement over the literature value. This new technique - threshold ion-pair production spectroscopy (TIPPS) - should be applicable to polyatomics, for which many bond energies are only known thermochemically.
\end{abstract}


Acknowledgments: I simply could not of had a better supervisor for this work than John Hepburn. I can't explain why he was such a good supervisor, although he is certainly helpful and knowledgeable. I never felt restricted to express certain opinions, or that dissent would be punished; debate was encouraged, which is a great atmosphere for doing science. John has an optimism and belief in people and certain things that sometimes seems unfounded or irrational; but this is exactly where these sentiments can have the most effect. Us seeing the $N=3$ line of the $\mathrm{O}_{2}$ TIPPS spectrum is one of the happiest moments I can remember.

I was lucky to interact with some really good people who worked in the XUV lab. Joining the group was exciting, because of the thesis results of Wei Kong, whose superb apparatus I used extensively during my work. Andreas Mank provided much help and guidance when I was learning about lasers, vacuum, electronics etc., and was an early advocate of the ion-pair experiment. Christian Alcaraz rebuilt much of the VUV generation system and was a great person to share an office and do experiments with.

The apparatus for these experiments was constructed in the University Science Shops, under the excellent direction of $\mathrm{C}$. Gendron - who made many suggestions concerning the design of the spectrometer and other equipment built for this work. The quality of craftsmanship, and helpfulness of the machinists was very much appreciated.

I'm grateful to Zulf Morbi for reading the draft of the oxygen ion-pair paper, making several useful suggestions, and interesting conversations during the time in which the oxygen experiments were done.

The Beams group was a great place to work - but it didn't seem like work - because of the many friends, colleagues and professors (not mutually exclusive!) with whom to discuss and exchange ideas. I remember with fondness many of the good times and talks with A. Beatty, L. Beauchamp, A. Bertram, M. Dulick, J. Guo, D. Lacombe, K. T. Leung, E. Luttmann, C. MacPherson, G. McGuire and R. Shiell.

I especially wish to thank my parents, Jim and Anne, for their help; and my father for his advice and comments on this work.

$$
\text { - J.D.D.M., Sept. 27, 1998, Upton, NY }
$$


The author may be reached at:

Department of Chemistry, 555A

Brookhaven National Laboratory

Upton, NY

11973

phone voice: 516-344-4339, fax: 516-344-5815

jmartinealbacore.chm.bnl.gov 


\section{Contents}

1 Introduction 1

1.1 Rydberg and Ion-Pair States $\ldots \ldots \ldots \ldots \ldots$

1.2 Photoionization and Photoion-pair Formation in Electric-Fields . . . . 7

1.3 Pulsed Electric-Field Induced Ionization and Dissociation . . . . . . 11

1.4 Molecular Rydberg States and Pulsed-Field Ionization Zero-Kinetic-Energy Photoelectron Spectroscopy . . . . . . . . . . . . . 15

1.5 Threshold Ion-Pair Production Spectroscopy $\ldots \ldots \ldots \ldots \ldots$

1.5.1 Photoion-pair Formation and Photoexcitation to high-v Ion-Pair

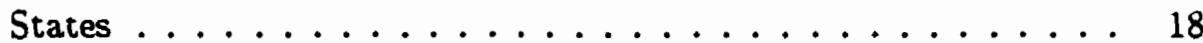

1.5.2 Threshold Photoion-pair Production Spectroscopy and the Determination of Dissociation Energies . . . . . . . . . . . . 24

2 Experimental 29

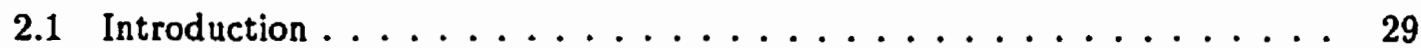

2.2 Generation of Vacuum Ultraviolet Radiation . . . . . . . . . . . 30

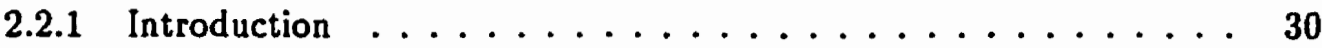

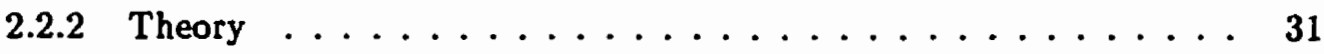




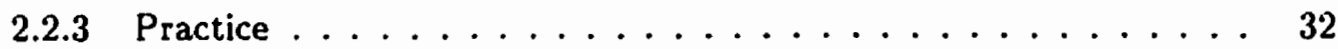

2.2 .4 Dye Lasers $\ldots \ldots \ldots \ldots \ldots \ldots \ldots$

2.3 Molecular Beam ...................... 36

2.4 Spectrometer ........................ 40

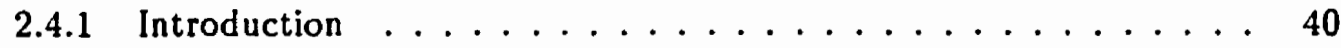

2.4 .2 The Kinematics of Discrimination . . . . . . . . . . 40

2.4.3 Field-Ionization and Extraction ..............443

2.4.4 Detection Electronics ................... 44

3 Mass Analyzed Threshold Ionization Spectroscopy of Argon - An Illustrative Example $\quad 46$

3.1 Introduction ....................... 46

3.2 Experimental ........................... 48

3.3 MATI Determination of the ${ }^{2} P_{1 / 2}$ Ionization Threshold of Ar ..... 49

3.4 Rydberg State Extrapolation to Determine the ${ }^{2} P_{1 / 2}$ Ionization Threshold of $\mathrm{Ar} \ldots \ldots \ldots \ldots \ldots \ldots \ldots \ldots \ldots$

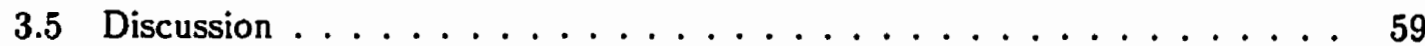

4 Threshold Ion-Pair Spectroscopy of $\mathrm{HCl}$

4.1 Introduction ........................ 61

4.2 Mechanism for the Formation of Ion-Pairs . . . . . . . . . . . 62

4.3 Experimental ......................... 64

4.4 Results and Discussion . . . . . . . . . . . . . . 65

4.5 Determination of $D_{0}(\mathrm{HCl}) \quad \ldots \ldots \ldots \ldots \ldots \ldots$ 
4.5.1 Extrapolation to Determine the Field-Free Ion-Pair Dissociation Threshold ........................ 74

4.5 .2 Isotopomer Correction . . . . . . . . . . . 76

4.5.3 Current Literature Value for $D_{0}(\mathrm{HCl}) \ldots \ldots . \ldots 1$

5 Threshold Ion-Pair Spectroscopy of $\mathrm{O}_{2} \quad 86$

5.1 Introduction ........................ 86

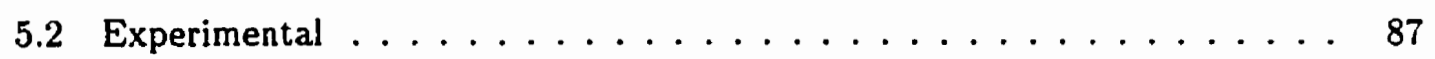

5.3 Results and Discussion . . . . . . . . . . . . . 88

6 Threshold Ion-Pair Spectroscopy of HF 96

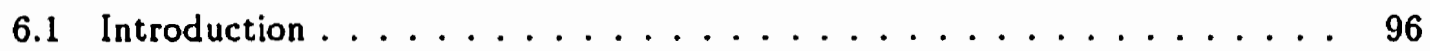

6.2 Experimental ............................. 98

6.3 Results and Discussion . . . . . . . . . . . . . . . . 98

6.4 Determination of $D_{0}(\mathrm{HF}) \ldots \ldots \ldots \ldots$

6.4.1 Extrapolation to Determine the Field-Free Ion-Pair Dissociation

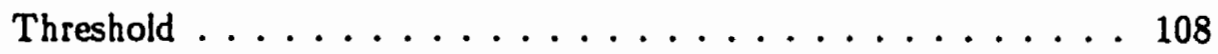

6.4.2 Current Literature Value for $D_{0}(\mathrm{HF}) \ldots \ldots \ldots \ldots$

7 Concluding Remarks $\quad 114$

A Wavelength Calibration Procedure $\quad 116$

A.1 Introduction ...................... 116

A.2 Observation of Uranium Optogalvanic Transitions . . . . . . . . 117

A.3 Effects of Air Temperature and Pressure on the Calibration . . . . . . 121

A.4 Scan Linearization ............................ 125 
B The Efficiency of High- $n$ Rydberg-State Stabilization in Pulsed-Field Ionization Zero-Kinetic-Energy Photoelectron Spectroscopy

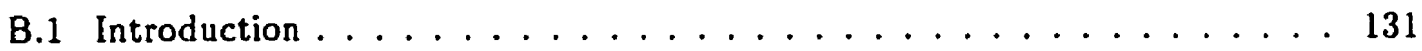

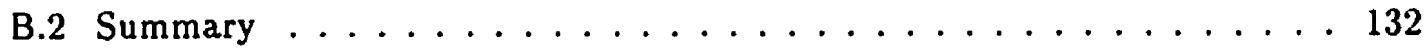

B.3 Historical Background . . . . . . . . . . . . . . 132

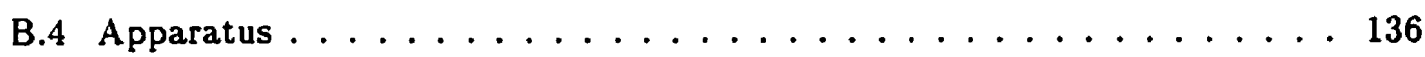

B.5 Results and Discussion . . . . . . . . . . . . 140

B.5.1 Cross-section Measurements . . . . . . . . . . . 140

B.5.2 Quantum Yield . . . . . . . . . . . . . . . 143

B.5.3 Lineshape Variations with Ion-Density . . . . . . . . . . . . 146

B.5.4 Lifetime Measurements and a Lineshape Model . . . . . . . . 151

B.6 Significance and Relationship to Other Work . . . . . . . . . . . 154

$\begin{array}{ll}\text { References } & 158\end{array}$ 


\section{List of Tables}

1.1 A brief survey of experimental photoion-pair formation studies. . . . . . 20

3.1 Line positions of the $3 p^{5} n s^{\prime}\left(\frac{1}{2}\right)_{1}^{0} \leftarrow 3 p^{6}{ }^{1} S_{0}$ Rydberg series of argon. . . . 58

5.1 Rotational energy levels of $\mathrm{O}_{2} \ldots \ldots \ldots \ldots \ldots$ 


\section{List of Figures}

1.1 Potential energy curves for HF. . . . . . . . . . . 2

1.2 Quantum defect as a function of vibrational quantum number for the $\mathrm{HF}$

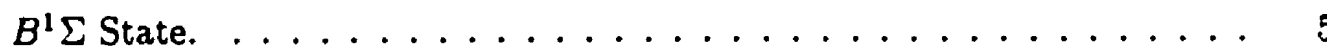

1.3 The effective long-range potential for a Rydberg electron with and without application of an electric field. . . . . . . . . . . . . 9

1.4 Experimental observations of the electric-field induced lowering of the energetic threshold for producing ion-pairs. . . . . . . . . . . . . 10

1.5 Threshold photoion-pair formation from $\mathrm{O}_{2} \ldots \ldots \ldots \ldots 22$

1.6 Energetics of ion-pair formation in $\mathrm{O}_{2} \ldots \ldots \ldots \ldots$

1.7 Formation of a centrifugal barrier . . . . . . . . . . . 25

2.1 Schematic illustration of the discrimination capability of the spectrometer for the $\mathrm{O}_{2}$ TIPPS experiment. . . . . . . . . . . . . . 41

2.2 Schematic diagram of spectrometer . . . . . . . . . . . . 45

3.1 MATI spectrum of Ar at the ${ }^{2} P_{1 / 2}$ ionization threshold. . . . . . . 50

3.2 MATI spectra of Ar at the ${ }^{2} P_{1 / 2}$ ionization threshold taken with different discrimination fields $\left(F_{d}\right) \ldots \ldots \ldots \ldots$ 
3.3 Extrapolation to determine the field-free ${ }^{2} P_{1 / 2}$ ionization threshold of Ar. $\quad 55$

3.4 $\mathrm{Ar}^{+}$total ion yield between the first and second ionization thresholds. $\quad$. $\quad 56$

3.5 Rydberg-state extrapolation to determine the ${ }^{2} P_{1 / 2}$ ionization threshold of Ar. ......................... 57

4.1 Potential energy curves of $\mathrm{HCl}$ and $\mathrm{HCl}^{+} \ldots \ldots \ldots 63$

4.2 Prompt photoion-pair yield from $\mathrm{HCl} \ldots \ldots \ldots \ldots$.......... 67

4.3 TIPPS spectra of $\mathrm{HCl}$ for two different discrimination fields. . . . . . . 69

4.4 $\mathrm{HCl}$ TIPPS spectra taken with a $15 \mathrm{~V} / \mathrm{cm}$ and $100 \mathrm{~V} / \mathrm{cm}$ Extraction Field. 70

$4.5 \mathrm{HCl}$ TIPPS spectra for different pulsed field extraction delays. . . . . . 73

4.6 $\mathrm{HCl}$ TIPPS spectra taken under different ion-density conditions. $\ldots . . .75$

4.7 Extrapolation to determine the field-free ion-pair formation threshold for HCl. ............................ 77

4.8 Model for the composite blue-edge line-shapes due to the two isotopomers of $\mathrm{HCl} . \ldots \ldots \ldots \ldots \ldots \ldots \ldots$

5.1 Prompt photoion-pair yield from $\mathrm{O}_{2} \ldots \ldots \ldots \ldots$

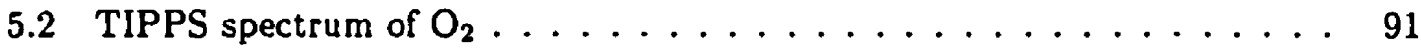

5.3 TIPPS spectrum of $\mathrm{O}_{2} \ldots \ldots \ldots \ldots \ldots \ldots$

6.1 Prompt photoion-pair yield from HF . . . . . . . . . . . . 99

6.2 TIPPS spectrum of $\mathrm{HF} \ldots \ldots \ldots \ldots \ldots$

6.3 $\mathrm{H}^{+}$prompt ion-yield and TIPPS spectrum of HF at the $J=0$ threshold. 103

6.4 $\mathrm{H}^{+}$prompt ion yield and TIPPS spectrum of HF at the $J=1$ threshold. 104

6.5 $\mathrm{H}^{+}$prompt ion yield and TIPPS spectrum of HF at the $J=2$ threshold. 105 xiii 
6.6 $\mathrm{H}^{+}$prompt ion yield and TIPPS spectrum of $\mathrm{HF}$ at the $J=3$ threshold. 106

6.7 $\mathrm{H}^{+}$prompt ion yield and TIPPS spectrum of HF at the $J=4$ threshold. 107

6.8 TIPPS spectra of HF for two different discrimination fields. . . . . . . 109

6.9 Extrapolation to determine the field-free ion-pair formation threshold for HF. ............................... 110

A.1 Circuit for observation of optogalvanic effect. . . . . . . . 118

A.2 Sample U optogalvanic spectrum ... . . . . . . . . . 120

A.3 Distribution of daily pressures in room C2-066 . . . . . . . . 123

A.4 Sample wavelength calibration using $U$ and Ne optogalvanic transitions . 127

A.5 Sample etalon pattern and $U$ optogalvanic spectrum . . . . . . 128

A.6 Sample calibration using etalon fringes. . . . . . . . . . 130

B.1 Photoionization cross-section of argon near the first ionization threshold. 142

B.2 Quantum yield measurements. . . . . . . . . . . . . . 144

B.3 Effects of ion-density on the PFI-ZEKE spectra of argon at the ${ }^{2} P_{1 / 2}$ limit: high ion densities. . . . . . . . . . . . . . . 148

B.4 Effects of ion-density on the PFI-ZEKE spectra of argon at the ${ }^{2} P_{1 / 2}$ limit: low ion densities. ....................... 149

B.5 Effects of ion-density on the PFI-ZEKE spectra of argon at the ${ }^{2} P_{3 / 2}$ limit. 150

B.6 PFI-ZEKE signal decay at $11 \mathrm{~cm}^{-1}$ below the ${ }^{2} P_{1 / 2}$ ionization threshold of argon. ........................ 153 


\section{Chapter 1}

\section{Introduction}

\subsection{Rydberg and Ion-Pair States}

In certain electronic states of diatomic molecules, the two constituent atoms behave like oppositely charged ions, since their mutual attraction is best described by the coulomb force law. One of these so-called "ion-pair" states is illustrated in Fig. 1.1, where the experimentally-derived potential energy curves of the ground and first electronic states of HF are plotted as a function of the internuclear separation. Also superimposed is a plot of the coulomb potential. As seen, the behavior of the $B$ ion-pair state is coulombic, but this behavior breaks down at low internuclear distance, when the atoms begin to touch.

In atomic and molecular physics we are more used to seeing the coulomb force in another context: the attraction between the electron and ion-core in a Rydberg atom. The ubiquitous example is the hydrogen atom; here the coulomb force law holds exactly until very small separation between the proton and electron, yielding the (non-relativistic) energy levels $(E)$ :

$$
E=-\frac{\mathcal{R}}{n^{2}}
$$




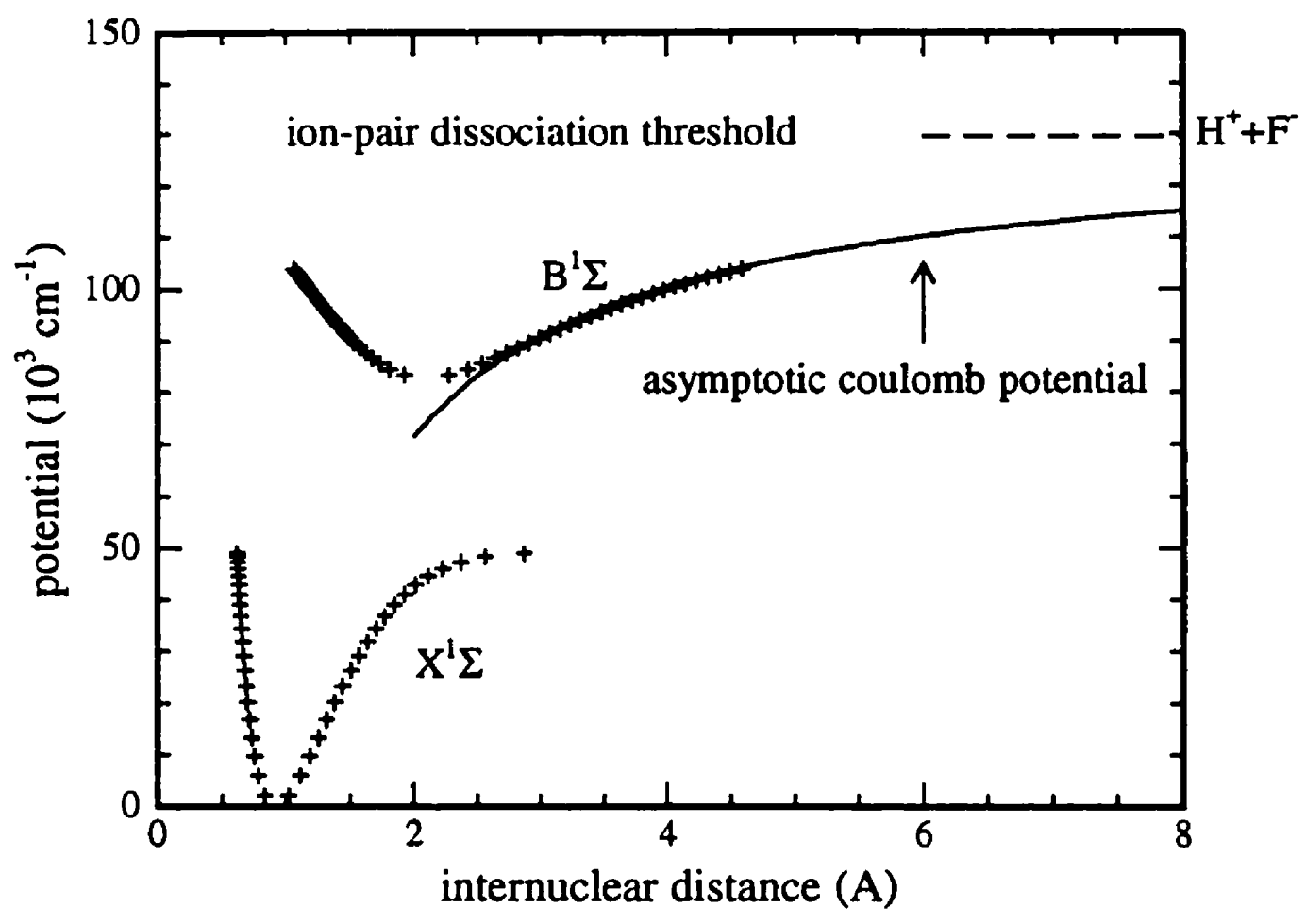

Figure 1.1: Potential energy curves for HF. The points shown are the Rydberg-Klein-Rees (RKR) turning points, computed by DiLonardo and Douglas [1] from their spectroscopic observations of the $B^{1} \Sigma-X^{1} \Sigma$ electronic system. (The RKR procedure is a method for "inverting" spectroscopic data to obtain potentials.) A coulomb potential has been superimposed (solid line). Its asymptotic energy has been chosen to correspond to the experimentally derived threshold for ion-pair formation, presented in Chapter 6 (129558 $\mathrm{cm}^{-1}$ ). 
where $n=1,2,3, \ldots$ and

$$
\mathcal{R}=\frac{m}{m_{e}} \mathcal{R}_{\infty}
$$

The quantity $m_{e}$ is the mass of the electron, $m$ is the reduced mass of the electron-proton two-body system, $m=m_{e} /\left(1+m_{e} / m_{p}\right)$, and $\mathcal{R}_{\infty}$ is the Rydberg constant $(\approx 13.6 \mathrm{eV}$, see Ref. [2] for a more precise value). Since the electron is much lighter than the proton, $\mathcal{R} \approx \mathcal{R}_{\infty}$. The reader will recall that states of different orbital angular momentum, $\ell$, have degenerate energies.

For atoms other than hydrogen, states in which one electron is highly excited and circles an ion-core, are similar to those of the hydrogen atom, and are known as Rydberg states. However, the influence of the electrons in the ion-core causes the purely coulombic behavior to break down at small separations between the Rydberg electron and the ioncore. This may be accommodated for, in the energy level formula, by the introduction of "quantum-defects" $\left(\delta_{l}\right): 1$

$$
E=-\frac{\mathcal{R}}{\left(n-\delta_{l}\right)^{2}}
$$

The quantum defects normally vary so smoothly with energy, (or $n$ ), that they may be assumed to be constant. The $\ell$-degeneracy is broken when the potential is not purely coulombic, so both quantum defects and energy levels are $\ell$-specific.

The ease with which non-hydrogenic Rydberg atom energy levels fit the modified Rydberg formula in Eqn. 1.3, suggests that a similar formula might be applicable to the vibrational energy levels of ion-pair states, which also show non-coulombic behavior at small separations. Specifically, one might expect: ${ }^{2}$

$$
E_{v}=-\frac{\mathcal{R}}{\left(v+1+J-\delta_{J}\right)^{2}}
$$

\footnotetext{
${ }^{1}$ The Rydberg constant must now be scaled by the reduced mass of the electron, ion-core two-body system.

${ }^{2}$ The denominator of Eqn. 1.4 is slightly more complicated, than that of Eqn. 1.3, since $v$ labeling starts at 0 , independent of $J$, whereas $n$ labeling for Rydberg states starts at $\ell+1$. Defining $\delta$, by Eqn. 1.4 retains an exact correspondence between quantum defects for Rydberg states and ion-pair states.
} 
where $\mathcal{R}$ is now $\mathcal{R}_{\infty}$, scaled by the reduced mass of the two circling ions. For the HF $B^{1} \Sigma$ state:

$$
\mathcal{R}=\left(\frac{1}{m_{e}}\right) \frac{m_{\mathrm{H}^{+}} m_{\mathrm{F}^{-}}}{m_{\mathrm{H}^{+}}+m_{\mathrm{F}^{-}}} \mathcal{R}_{\infty}
$$

where $m_{\mathrm{H}^{+}}$is the mass of the proton, and $m_{\mathrm{F}}$ is the mass of the fluorine anion. Figure 1.2 shows the quantum defect of the vibrational energy levels of the $B$ ion-pair state of $\mathrm{HF}$ as a function of vibrational quantum number, computed using Eqn. 1.4. As the vibrational quantum number increases, the quantum defect varies less, thus Eqn. 1.4 becomes a more accurate description of the vibrational energy level spacing. Most importantly, there are an infinite number of vibrational levels below the ion-pair dissociation threshold. This is unusual, as it may be shown that only potentials having an asymptotic form:

$$
V(R) \stackrel{R \rightarrow \infty}{=}-\frac{C}{R^{\alpha}}
$$

where $0<\alpha \leq 2$, can exhibit this property [3]. The ion-pair states satisfy this relationship. More typical long-range interactions are of the form $1 / R^{6}$ (van der Waals interaction) and $1 / R^{4}$ (ion-neutral interaction) [4]. These "weaker" long-range potentials only support a finite number of vibrational levels.

The correspondence between Rydberg-state energy level spacings and ion-pair state vibrational energy level spacings has been noted previously by Asaro and Dalgarno [6] and Pan and Mies [?].

In addition to the similarity of their energy level formulae, Rydberg states and ionpair states are expected to share other properties. One example is size, which for Rydberg atoms may be defined as the expectation value of the electron, ion-core separation $\langle r\rangle$. For hydrogen, in an $\ell=0$ state [8]:

$$
<r>=\frac{3}{2} n^{2}
$$

This is given in atomic units, for which the unit of length is the Bohr radius, $a_{0}=0.529$ $\AA$. Equation 1.7 is exactly true for hydrogen, and approximately true for all Rydberg 


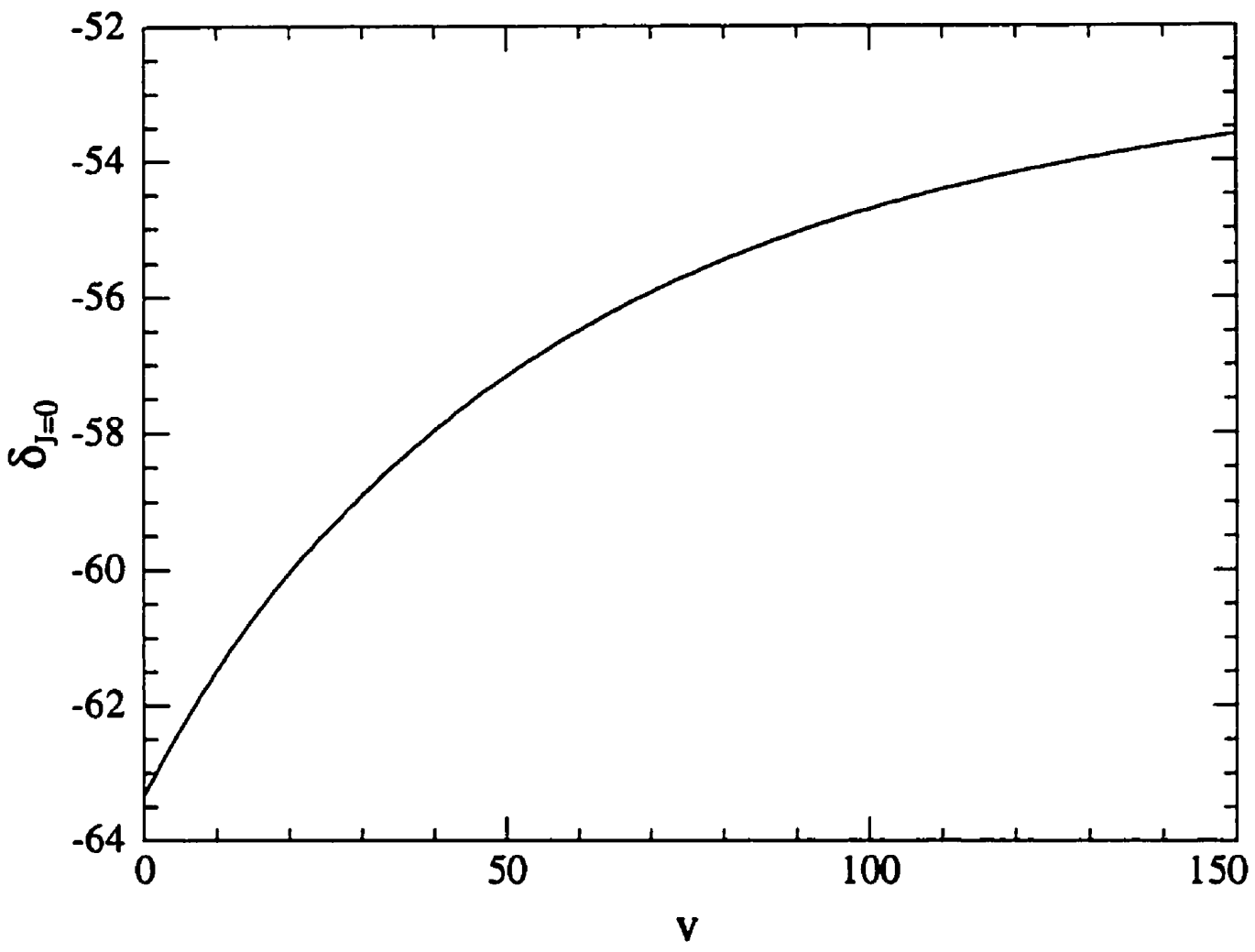

Figure 1.2: Quantum defect $(\delta)$ as a function of vibrational quantum number $(v)$ for the levels of the HF $B^{1} \Sigma$ state potential. The potential was approximated using cubic-spline interpolation between DiLonardo and Douglas's RKR points [1], with suitable extensions for $R<1.0648 \AA$ and $R>4.5890 \AA$. To obtain the vibrational energy levels, the NumerovCooley technique was used [5]. 
atoms. To scale these results to ion-pair systems, it is convenient to introduce a set of "generalized atomic units", denoted by the subscript $g$ :

$$
\begin{gathered}
a_{g}=\frac{1}{m} \frac{\hbar^{2} 4 \pi \epsilon_{0}}{q_{e}^{2}} \\
E_{g}=m \frac{q_{e}^{4}}{\hbar^{2}\left(4 \pi \epsilon_{0}\right)^{2}} \\
F_{g}=m^{2} \frac{q_{e}^{5}}{\hbar^{4}\left(4 \pi \epsilon_{0}\right)^{3}}
\end{gathered}
$$

where $a_{g}, E_{g}$, and $F_{g}$ are the units of length, energy and electric field, respectively. ${ }^{3}$ The rationalized MKSA system has been used for the electromagnetic quantities: $q_{e}$, the elementary charge, and $\epsilon_{0}$, the permittivity of vacuum. For normal atomic units $m=m_{e}$, the mass of the electron, and in this case, the normal atomic units are recovered:

$$
\begin{aligned}
a_{g} & \approx 5.29 \times 10^{-11} \mathrm{~m} \\
E_{g} & \approx 4.36 \times 10^{-18} \mathrm{~J} \\
F_{g} & \approx 5.14 \times 10^{11} \mathrm{~V} / \mathrm{m} .
\end{aligned}
$$

For ion-pair states, $m$ is the reduced mass of the two-body system, and the corresponding generalized units differ from the normal atomic ones. Formulae written in atomic units for Rydberg states may be considered applicable to ion-pair states, if they are converted to real units using the appropriate generalized units. To convert an equation written in atomic units to real units, appropriate for ion-pair states, replace all instances of energy $(E)$ by $E / E_{g}$, lengths $(l)$ by $l / a_{g}$, electric fields $(F)$ by $F / F_{g}$, etc.

For example, Eqn. 1.7 may be applied to ion-pair states:

$$
<r>=\frac{1}{m} \frac{\hbar^{2} 4 \pi \epsilon_{0}}{q_{e}^{2}} \frac{3}{2} v^{2} .
$$

The quantity $\langle r\rangle$ now represents the separation of the two ions. From this, it can be seen that ion-pair states will be smaller than Rydberg states, for $v \approx n$, since the reduced

\footnotetext{
${ }^{3}$ The generalized atomic units for time, and other quantities, can be determined by appropriate modification of the quantities listed by Bethe and Salpeter, on pg. 3 of Ref. [8].
} 
masses of ion-pair systems are always larger than the mass of an electron. However,

Rydberg and ion-pair states with the same binding energies, $v \approx n\left(\frac{m}{m_{e}}\right)^{1 / 2}$, will have approximately equal sizes.

Since one can easily produce Rydberg states of $n=100$, their size can approach laboratory scales. In particular, Fabre and co-workers [9] have monitored the depletion of a beam of Rydberg atoms sent through an electro-formed mesh, as a function of the size of the holes and the $n$ of the Rydberg states. They confirm that the scaling of size is given by Eqn. 1.7. Experiments with ion-pair states of equivalent binding energies would be expected to show similar results.

The large size of Rydberg states is only one of their extreme characteristics. Since the invention of the tunable dye laser in the 1970's, the study of Rydberg states has undergone a renaissance, and many other properties and behavior of these "exaggerated atoms" [10] have undergone extensive study. Two books $[10,11]$ discuss many of these experiments.

What other properties do the Rydberg and ion-pair states have in common? In this thesis it is demonstrated that highly vibrationally excited ion-pair states can be dissociated with weak electric fields, in a similar manner to the well-studied electric-field induced ionization of high-n Rydberg states.

\subsection{Photoionization and Photoion-pair Formation in Electric- Fields}

With an electric-field present, the potential for the motion of a Rydberg electron is no longer coulombic, nor spherically symmetric. In atomic units, the long-range potential is:

$$
V(R, z)=-\frac{1}{R}-F z
$$

where $-z$ is the direction of the applied electric-field, $F$. Figure 1.3 illustrates this potential along the electric-field axis. As can be seen, application of the electric field 
lowers the energy of the ionization threshold. The lowering, $\Delta E$, is given by [10]:

$$
\Delta E=2 \sqrt{F}
$$

or in real units,

$$
\Delta E=6.1 \frac{\mathrm{cm}^{-1}}{(\mathrm{~V} / \mathrm{cm})^{1 / 2}} \sqrt{F} .
$$

This lowering of the ionization threshold with electric-field may be confirmed by observing the shift in energy required to photoionize the atom. Varying the size of a DC electricfield in the photoexcitation volume lowers the photon energy required for ionization, in a manner determined by Eqn. 1.14 (see, for example, Ref. [12]).

This simple viewpoint - known as the classical saddle point model - is very useful. There are sometimes complications; states with non-zero $m_{\ell}$ can be kept away from the classical saddle point by centrifugal forces [13], and it may be necessary to consider the effects of tunneling through the barrier. However, these are often not significant.

Since the lowering of the ionization threshold described by Eqn. 1.14 is a purely electrostatic effect, and in no way depends on electron mass, one would expect that similar considerations apply to ion-pairs. For example, the threshold for: $\mathrm{H}_{2}+h \nu \rightarrow \mathrm{H}^{+}+\mathrm{H}^{-}$ can be reduced by the application of a DC field. This was first confirmed experimentally by Pratt and co-workers in 1992 [14]. They excited molecular hydrogen from its ground electronic state to $v=6$ of the $E F^{1} \Sigma_{g}^{+}$state using two photons. A third tunable photon was then used to excite to the ion-pair threshold. Scanning this final photon energy, and monitoring free ion-pair production, the energetic threshold for ion-pair formation could be observed. This is shown in Fig. 1.4a). There is a clearly observable threshold. If the DC field in the photoexcitation region is changed, this threshold moves. Figure 1.4b) shows the threshold energy plotted as a function of the square root of the electric-field. As expected from Eqn. 1.14, the experimental points fall on a straight line, with a slope very close to the expected value of $6.1 \mathrm{~cm}^{-1} /(\mathrm{V} / \mathrm{cm})^{1 / 2}$. 


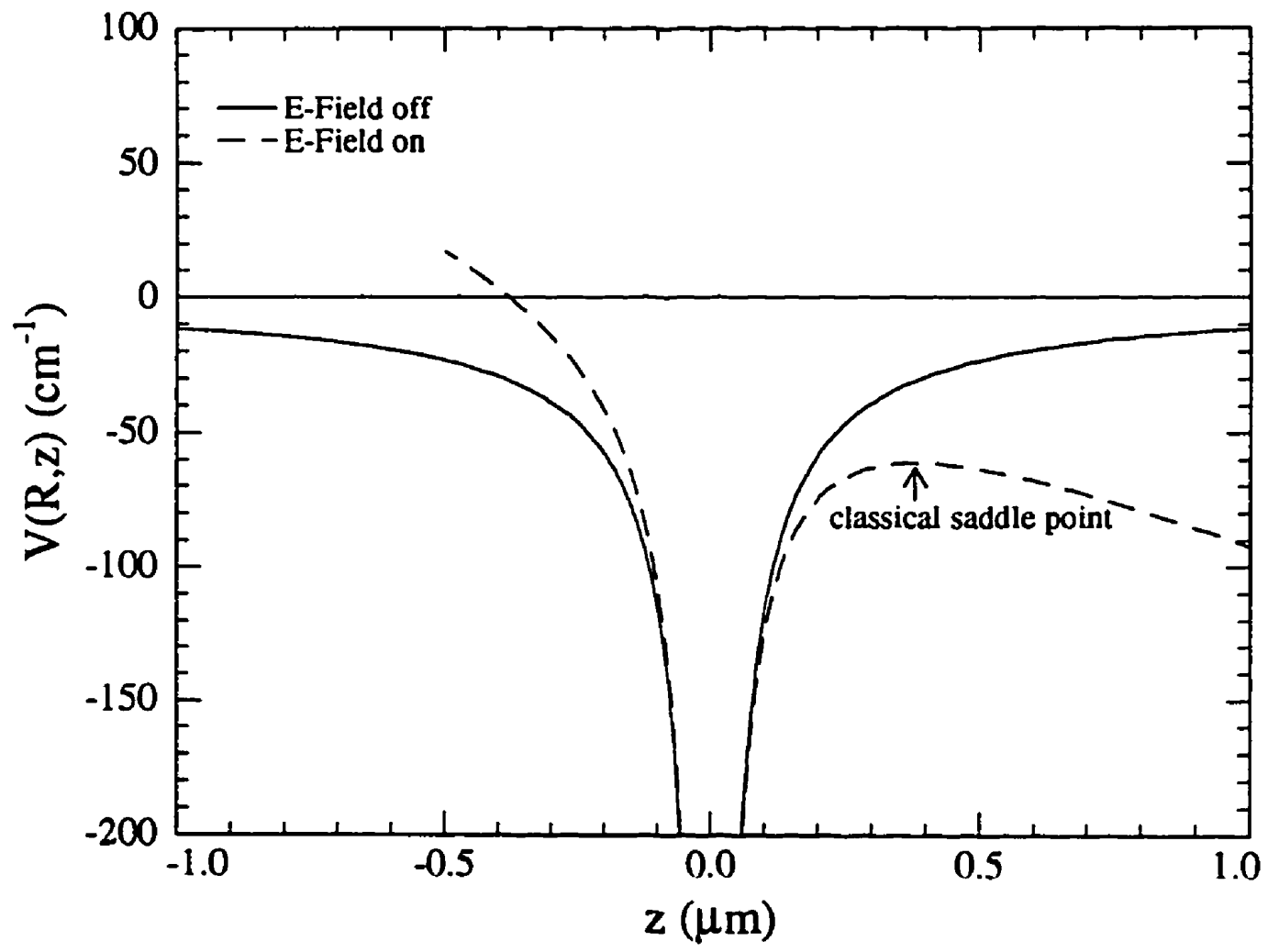

Figure 1.3: The effective potential for a Rydberg electron with and without application of an electric field. Field strength is $100 \mathrm{~V} / \mathrm{cm}$. 

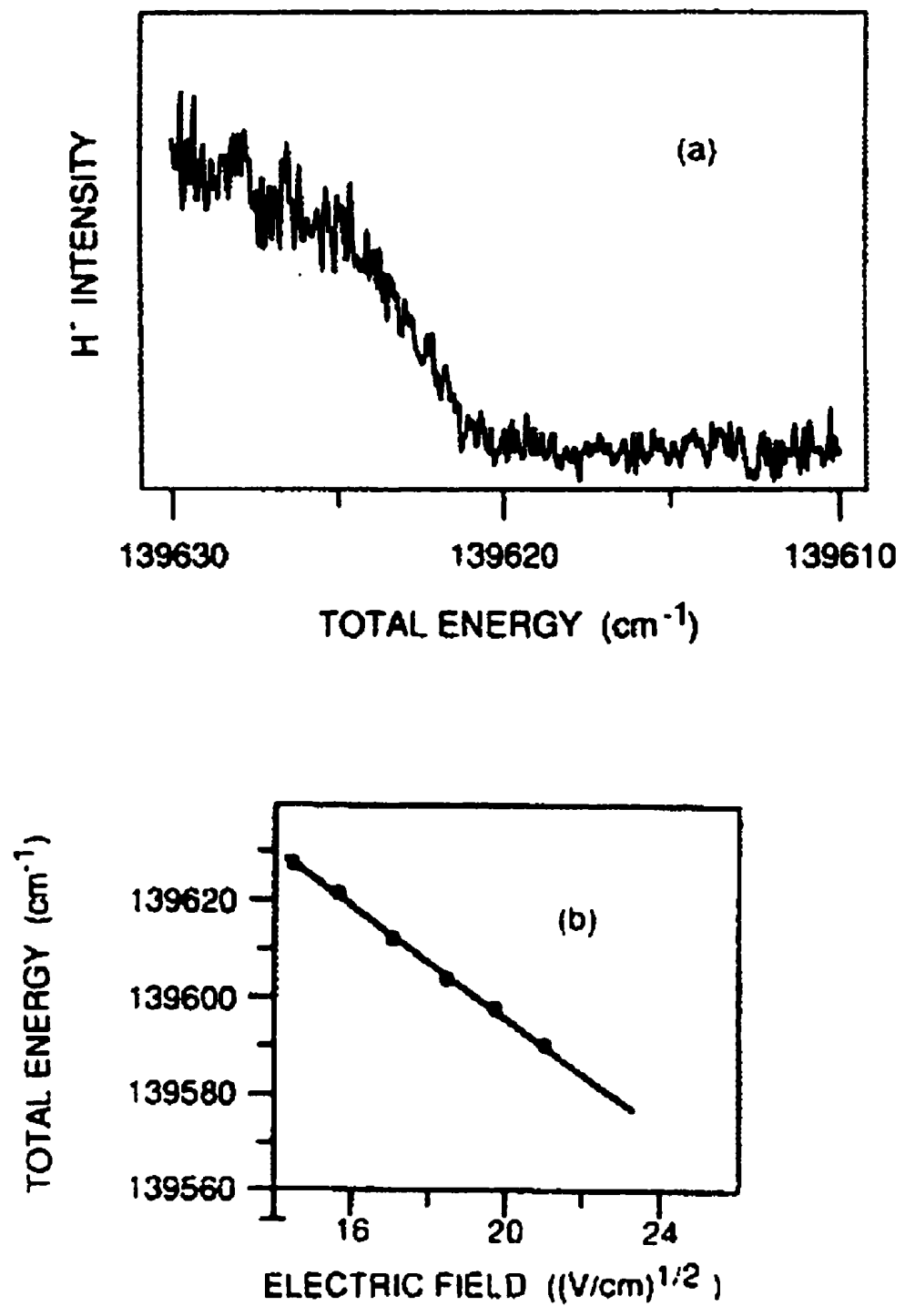

Figure 1.4: Experimental observations of the electric-field induced lowering of the energetic threshold for producing ion-pairs. From Ref. [14]. a) The $\mathrm{H}^{-}$ion-signal produced from $\mathrm{H}_{2}$ as a function of energy above the $X^{1} \Sigma_{g}^{+}, v=0, J=0$ ground state, with a DC field of $245.7 \mathrm{~V} / \mathrm{cm}$. The spectrum was obtained by pumping the $Q(1)$ $E F^{1} \Sigma\left(v^{\prime}=6, J^{\prime}=1\right) \leftarrow X^{1} \Sigma_{g}^{+}\left(v^{\prime \prime}=0, J^{\prime \prime}=1\right)$ two-photon transition. b) The energetic thresholds for ion-pair formation as a function of the square root of the electric field. The energy is with respect to the $X^{1} \Sigma_{g}^{+}, v=0, J=0$ ground state. The fitted line has a slope of $5.7 \pm 0.2 \mathrm{~cm}^{-1} /(\mathrm{V} / \mathrm{cm})^{1 / 2}$. 


\subsection{Pulsed Electric-Field Induced Ionization and Dissocia- tion}

Thus far, photoexcitation in electric-fields has been discussed. What happens when an electric-field is applied to an already existing Rydberg state? Not surprisingly, it may "field-ionize", producing a free-electron and remaining ion-core. The magnitudes of the fields required to ionize Rydberg states will be discussed in this section, with a view towards studying analogous behavior for high-v ion-pair states.

As soon as an electric-field is applied, Rydberg state energies begin to shift; this is the Stark effect. Spherical symmetry is lost $-\ell$ ceases to be a good quantum number - as the electric field mixes different $\ell$ states. For non-relativistic hydrogen, the highly degenerate zero-field states fan out into a "manifold" for each $m_{\ell}$ - which remains a good quantum number. At low fields $(F)[8,10]$ :

$$
E=-\frac{1}{2 n^{2}}+\frac{3}{2} F\left(n_{1}-n_{2}\right) n
$$

where $n_{1}$ and $n_{2}$ are non-negative integers labeling different members of the manifold, constrained by the relationship $n_{1}+n_{2}+\left|m_{\ell}\right|+1=n$. For $m_{\ell}=0$, the extreme "blueshifted" state of the manifold has $n_{1}-n_{2}=n-1$, while the extreme "red-shifted" state has $n_{1}-n_{2}=-n+1$. The energy separation between these extreme states of the manifold increases with $n$. Similarly, the ground states of non-hydrogen atoms show small shifts with increasing fields (compared to their high- $n$ Rydberg states). High $m_{\ell}$ states of nonhydrogenic atoms are also described by Eqn. 1.15, as they have little penetrating, low- $\ell$ character.

As electric-field is increased from zero, blue-shifted members originating from one value of $n$ will approach the energy of red-shifted states of high- $n$. This gives rise to "avoided-crossings"; as the field strength is increased, two quantum states approach one another in energy, then recede. An atom in one state may "jump" to another, if this avoided crossing is approached rapidly (diabatic). If the crossing is approached slowly 
(adiabatic) the atom is more likely to stay in the same state. Intermediate behavior is also possible. This is a consequence of time-dependent quantum mechanics, and is frequently modeled with the Landau-Zener theory $[15,16]$.

The practical consequence is that a field-ionizing pulse may shift a Rydberg state up or down in energy with respect to the ground state of the atom. Consequently, it is no longer possible to simply use the classical saddle point model, for predicting the field at which a Rydberg state will ionize. There is an exception; if the field is applied slowly enough, such that all avoided crossings are traversed adiabatically, field ionization occurs when the saddle-point energy equals the initial Rydberg state binding energy [10]. For completely adiabatic ionization, the critical ionization field strength, $F_{c}$, for a Rydberg state of initial $n$ is

$$
F_{c}=\frac{1}{16 n^{4}}
$$

or, rewriting this in terms of the binding energy of the initial field-free Rydberg state, $E=1 /\left(2 n^{2}\right)$,

$$
F_{c}=\frac{E^{2}}{4}
$$

When the electric-field is applied quickly, the avoided crossings are traversed diabatically. Using the hydrogen system as a model, the most extreme red-shifted states of a $m_{\ell}=0$ manifold will be at the classical saddle point energy when $F_{c}=1 /\left(9 n^{4}\right)$, where $n$ is the initial principal quantum number (see Ref.'s $[17,10]$ ). Or rewriting in terms of binding energy:

$$
F_{c}=\frac{4 E^{2}}{9}
$$

This predicts that a stronger field is required for ionization than in the adiabatic case (Eqn. 1.17).

Since the blue-shifted members of the Stark manifold will increase in energy, one might expect that they would be ionized at lower field strengths than the red-shifted Stark states; their energies will be above the classical saddle-point for ionization at much 
lower fields. However, this is not the case, as in these blue shifted states, the Rydberg electron wavefunction is localized on the side of the atom away from the lowered barrier (-ve $z$ in Fig. 1.3). Since the electron does not approach the barrier, it cannot ionize, even though this is energetically allowed. This is nicely illustrated in Fig. 3 of Merkt's review article [18]. Empirically, it is observed that the extreme blue shifted states take roughly twice the field strength to ionize, compared to the red-shifted states (see for example, Fig. 4 of Ref. [18]). The relationship: $F_{c}=1 /\left(4 n^{4}\right)$, is sometimes quoted for $m_{\ell}=0$ hydrogen [19]. Rewriting in terms of binding energy, the critical field for ionization is:

$$
F_{c}=E^{2}
$$

Again, the fields required for ionization are higher than for the adiabatic case.

If the diabatic ionization mode is operative, a mixture of states excited at a specific binding energy will be $100 \%$ ionized for a field strength above the blue-shifted limit (Eqn. 1.19), will be partially ionized for fields between the blue and red-shifted limits (Eqn. 1.19 and Eqn. 1.18), and remain completely un-ionized for field strengths below the red-shifted limit (Eqn. 1.18). For purely adiabatic ionization, field strengths above the classical limit (Eqn. 1.17) will be $100 \%$ efficient in ionization, and those below, completely inefficient. However, adiabatic and diabatic are two extremes. In practice it is possible to a mixture of both behaviors. This is illustrated in the work of Jeys and co-workers [20], who show Rydberg states ionized by both modes, but that the diabatic route is favored with increasing $n$.

This discussion of pulsed-field ionization has been necessarily brief and imprecise. However, the salient point is that the critical fields for ionization scale in proportion to the square of the binding energy:

$$
F_{c}=k E^{2} \text {. }
$$

for adiabatic $(k=1 / 4)$ and red $(k=4 / 9)$ and blue-shifted $(k=1)$ diabatic behavior. Converting this equation from generalized atomic to real units, as discussed in Section 
1.1, gives: ${ }^{4}$

$$
F_{c}=\frac{4 \pi \epsilon_{0}}{q_{e}^{3}} k E^{2} .
$$

This result is independent of the reduced mass of the system. Thus, the critical fields for the ionization of ion-pair states are predicted to be identical to those for Rydberg states of the same binding energy. 5

To the best of the author's knowledge, no previous investigations of the pulsed-field dissociation of high- $v$ ion-pair states have ever been made. However, Pratt and coworkers observations [14], together with what is known about Rydberg state ionization, suggested that the electric-field induced dissociation of high- $v$ ion-pair states was feasible. Observation rested upon choice of a suitable molecule and detection technique.

To investigate the electric-field induced dissociation of high-v ion-pair states one could repeat Pratt and co-workers experiment, using a pulsed field after photoexcitation. By varying the time-delay between excitation and the field-dissociation pulse, the lifetimes of high-v ion-pair states could be studied. Varying the size of the pulsed electric-field would test how well the various formulae for Rydberg-state ionization apply to the ion-pair case. This was our original plan, and a series of experiments were done to gain experience with double resonance experiments in $\mathrm{H}_{2}$ [21]. However, eventually it was decided to take another approach: to excite from the ground state of a molecule to energies just below the ion-pair dissociation threshold - hopefully populating high-v ion-pair states - and then use two electric-field pulses to selectively detect excitation into a range of high-v ion-pair states. This technique is both conceptually and practically similar to both pulsedfield ionization zero-kinetic-energy electron spectroscopy (PFI-ZEKE), and mass-analyzed

\footnotetext{
${ }^{1}$ Or in real units, $E=6.12 \frac{\mathrm{cm}^{-1}}{(\mathrm{~V} / \mathrm{cm})^{1 / 2}} \sqrt{F_{c}}, E=4.59 \frac{\mathrm{cm}-1}{(\mathrm{~V} / \mathrm{cm})^{1 / 2}} \sqrt{F_{c}}$, and $E=3.06 \frac{\mathrm{cm}^{-1}}{(\mathrm{~V} / \mathrm{cm})^{1 / 2}} \sqrt{F_{c}}$, for the adiabatic, red and blue-shifted diabatic cases respectively.

${ }^{5}$ However, which formulae apply, adiabatic or diabatic, should depend on binding-energy since high-v spacings are much more dense than high-n Rydberg state spacings for the same binding energy. Thus, avoided crossings are more likely to be traversed diabatically for high-v ion-pair states than for high-n Rydberg states of the same binding energy.
} 
threshold ionization spectroscopy (MATI) - two well-established techniques which involve the electric-field induced ionization of high- $n$ Rydberg states - so these are discussed in the next section.

\subsection{Molecular Rydberg States and Pulsed-Field Ionization Zero-Kinetic-Energy Photoelectron Spectroscopy}

The historical origins of PFI-ZEKE spectroscopy are a series of threshold photoelectron spectroscopy experiments on the NO molecule, by Müller-Dethlefs, Schlag and coworkers at the Technical University of Munich $[22,23]$. In conventional photoelectron spectroscopy, a fixed frequency light source irradiates molecules at a photon energy well above their ionization threshold (by several eV). The resulting photoelectrons are then energy analyzed. Electrons are emitted at different energies, as the resulting ion can be left in different rovibrational states (an ion left with a high amount of vibrational energy, say, will emit a slower photoelectron, simply due to conservation of energy). Thus the ionic energy levels can be determined by the energy spacings of the peaks in the photoelectron spectrum.

In threshold photoelectron spectroscopy, the light source is now scanned, and a filter is set up to detect only very slow photoelectrons. By monitoring this slow-electron signal as the photon energy is scanned over the different ionization thresholds, the ion energies may be determined. Conventional photoelectron spectroscopy also determines the ionic energy levels. However, with the threshold technique, the energy scale is obtained from the photon energy calibration, rather than from the generally more difficult electron kinetic energy analysis. There are other advantages to the threshold technique. It is easier to optimize a high-throughput, high-resolution detection of threshold electrons, compared with conventional photoelectron spectroscopy.

As discussed in Guyon and Baer's historical review [24], the PFI-ZEKE technique 
was discovered, as part of a program to improve the resolution of threshold photoelectron spectroscopy. In particular, in 1984, Müller-Dethlefs and co-workers [22] demonstrated a threshold photoelectron spectrum of NO with remarkable resolution, by using a pulsed excitation combined with a delayed extraction field, with the goal of enhancing the selectivity towards near-threshold electrons. The greatly improved resolution of this scheme allowed them to distinguish threshold electrons from ions left in different rotational states. The technique was named zero-kinetic energy photoelectron spectroscopy (ZEKE), to distinguish it from the other threshold techniques. Since the intensities of the ZEKE peaks were expected to indicate the relative probabilities for photoionizing the molecule and leaving the ion in different rotational states, obtaining rotational resolution was particularly significant from the point of view of understanding the physics of molecular photoionization. Previously, rotationally resolved photoelectron spectra had only been achieved for $\mathrm{H}_{2}[25,26]$, an exceptional case due to its widely separated rotational levels.

In the original ZEKE paper, a slight discrepancy was noted; the energetic ionization thresholds of the NO molecule may be accurately determined from an extrapolation of its observed Rydberg series, ${ }^{6}$ but it was found that the ZEKE signals were at energies slightly below $\left(4 \mathrm{~cm}^{-1}\right)$ these thresholds. Upon further analysis, it appeared that the waiting period was actually $100 \%$ efficient in discriminating against all above threshold photoelectrons, and the observed signal corresponded to the electric-field ionization of high- $n$ Rydberg states which had been excited by the laser pulse. This seemed reasonable, since series of high- $n$ Rydberg states approach each molecular ionization threshold.

This was confirmed by varying the strength of the electric field pulse $F$ and observing that larger fields broadened the spectral peaks to lower energies. Specifically, the low-energy sides of the peaks shifted down from the field-free ionization threshold, in proportion to $\sqrt{F}$. By taking several spectra at different fields, and extrapolating the results to zero-field, it was possible to determine the field-free ionization threshold. Agreement

\footnotetext{
'For molecules, unlike atoms, this extrapolation is normally difficult because of the extensive perturbations, or interseries couplings between Rydberg states approaching the different ionization thresholds.
} 
with the value determined by Rydberg series extrapolation was excellent.

Based on this new understanding of the process responsible for the very high resolution, Müller-Dethlefs and Schlag suggested a more appropriate designation for the technique [27]: pulsed-field ionization zero-kinetic-energy photoelectron spectroscopy, or PFI-ZEKE for short. However, workers often shorten this to "ZEKE", even if they are using the pulsed-field ionization technique. ${ }^{?}$

The use of the PFI-ZEKE technique has become widespread. It is especially useful for the accurate determination of molecular ionization energies ${ }^{8}$ and for certain ion spectroscopic work, not easily approachable by other means [28].

An important variant of the PFI-ZEKE technique was introduced by Zhu and Johnson in 1991 [29]. They demonstrated that it was possible to selectively detect the ions instead of the electrons - arising from field ionization. This is more difficult, since discrimination against the prompt ions is more difficult than for prompt electrons because of their mass difference. However, this may be accomplished by the introduction of a small electric field in the waiting period between excitation and field-ionization (by a larger field). If the photon energy is scanned and the ions originating from field-ionization are detected, the resulting spectra are equivalent to the PFI-ZEKE spectrum, with one important difference; the resulting ion may be mass analyzed (normally by time-of-flight), prompting the description: mass-analyzed threshold ionization spectroscopy (MATI). This technique is advantageous when working with cluster or radical sources, where the molecular beam may consist of different species.

In addition to the new spectroscopic information, attention has also been focused on using PFI-ZEKE to study the dynamics of the photoionization process. Earlier, it was

\footnotetext{
${ }^{7}$ Müller-Dethlefs and co-workers later showed that it was in fact possible to collect a "true" ZEKE spectrum, avoiding the signal due to pulsed-field ionization, as discussed in their review article [27]. Thus, dropping the PFI designation seems problematic, although it is common.

-A list of molecular ionization energies determined by PFI-ZEKE is available from the web-page: http://eos.phys.chemie.tu-auenchen.de/zeke/, and currently numbers 146.
} 
mentioned that ZEKE's access to rotationally resolved photoionization cross-sections was particularly exciting. Although the PFI-ZEKE signals arise from field-ionization of Rydberg states rather than from true threshold photoionization, it may still be legitimate to relate them to threshold photoionization cross-sections. The basis of this comparison is "continuity of oscillator strength". The reader is referred to the literature for a complete discussion (see, for example, Ref. [3]). In summary, the practical consequence is that the probability of exciting Rydberg states with a broadband source just below an ionization threshold, is identical to the probability of ionization just above the energetic threshold. 9 Thus PFI-ZEKE spectra which record the Rydberg state excitation probabilities just below each ionization threshold, have determined the relative photoionization cross-sections at threshold. However, loss of Rydberg states in the waiting period, due to autoionization or predissociation, can sometimes spoil this correspondence. This is discussed more extensively in Appendix B, where PFI-ZEKE signals at the ${ }^{2} P_{3 / 2}$ (first) and ${ }^{2} P_{1 / 2}$ (second) ionization thresholds of argon are compared to the well-established partial photoionization cross-sections.

\subsection{Threshold Ion-Pair Production Spectroscopy}

\subsubsection{Photoion-pair Formation and Photoexcitation to high-v Ion-Pair States}

The previous section mentioned the strong connection between excitation to the high-n Rydberg states below the ionization threshold, and the adjoining photoionization continuum. For excitation of highly vibrationally excited ion-pair states, there is also an associated continuum:

$$
\mathrm{AB}+h \nu \rightarrow \mathrm{A}^{+}+\mathrm{B}^{-} .
$$

\footnotetext{
${ }^{\circ}$ More precisely, this is the probability of ionization leaving an ion-core in the same state that the Rydberg series was converging on.
} 
This is sometimes referred to as "polar-photodissociation" [30], but is more commonly called "photoion-pair formation".

Typical experimental studies of photoion-pair formation characterize the yield, or cross-section, for the process as a function of photon energy. These yield spectra are typically rich in structure, since the process for ion-pair formation normally involves excitation to a Rydberg state, which then predissociates into ion-pairs:

$$
\mathrm{AB}^{+h} \nu \rightarrow \mathrm{AB}^{*} \rightarrow \mathrm{A}^{+}+\mathrm{B}^{-}
$$

The structure in the yield spectra is due to excitation to quasi-bound states in the first step. Direct excitation from the ground state to the ion-pair continuum seems to be rare.

The photoion-pair production process has been studied in many molecules, ${ }^{10}$ and some of these are summarized in Table 1.1. It is important to point out that processes such as $\mathrm{NaCl}+h \nu \rightarrow \mathrm{Na}^{+}+\mathrm{Cl}^{-}$, which one might expect to be quite strong, have never been observed. This is discussed more extensively in the review by Berkowitz [34].

Frequently, it is possible to observe strong photoion-pair formation directly at the energetic threshold. ${ }^{11}$ Dehmer and Chupka [37] have recorded a high resolution yield

\footnotetext{
${ }^{10}$ In addition to characterization of the total cross-section as a function of photon energy, there have been other types of experiments associated with photoion-pair formation. Many of these are the analogs of photoelectron spectroscopy. Photolysis is performed at a fixed photon energy, and the kinetic energies of the ions produced are analyzed (see, for example, Refs. [31] and [32]). For example, an interesting study has been performed by Munakata and Kasuya [33]. They observed the processes: $\mathrm{CH}_{3} \mathrm{Br}+h \nu \rightarrow$ $\mathrm{CH}_{3}^{+}+\mathrm{Br}^{-}$and $\mathrm{CH}_{3} \mathrm{Cl}+h \nu \rightarrow \mathrm{CH}_{3}^{+}+\mathrm{Cl}^{-}$, using $118 \mathrm{~nm}$ light, and analyzing the kinetic energies of the produced ions. They found that only a small fraction of the total available energy was released into kinetic energy ( $17 \%$ of $0.98 \mathrm{eV}$ for $\mathrm{CH}_{3} \mathrm{Br}$ and $25 \%$ of $0.69 \mathrm{eV}$ for $\mathrm{CH}_{3} \mathrm{Cl}$ ). The rest must go into $\mathrm{CH}_{3}^{+}$ vibrations. This is understandable, since the ground state of $\mathrm{CH}_{3}^{+}$is planar, in contrast to the strongly pyramidal shape of $\mathrm{CH}_{3}$ in the parent molecule. The dramatic geometry change deposits vibration in the $\mathrm{CH}_{3}^{+}$fragment, reducing the available energy.

${ }^{11}$ For photolysis into neutrals, Wigner's threshold laws [50] dictate that the cross-section must be zero at threshold (see, for example, Ref. [51]). These laws do not necessitate that the crose-section for ion-pair formation be zero at threshold - since the two departing bodies interact with the strong coulomb force.
} 


\begin{tabular}{|c|c|c|c|}
\hline Process & $\begin{array}{c}\text { Energetic Threshold } \\
(\mathrm{eV})\end{array}$ & $\begin{array}{c}\text { Ionization Potential } \\
(\mathrm{eV})\end{array}$ & References \\
\hline $\mathrm{HCl}+h \nu \rightarrow \mathrm{H}^{+}+\mathrm{Cl}^{-}$ & 14.42 & 12.75 & {$[35,36]$} \\
$\mathrm{O}_{2}+h \nu \rightarrow \mathrm{O}^{+}+\mathrm{O}^{-}$ & 17.27 & 12.07 & {$[37,38,39,40,41]$} \\
$\mathrm{HF}+h \nu \rightarrow \mathrm{H}^{+}+\mathrm{F}^{-}$ & 16.06 & 16.04 & {$[42,31,43]$} \\
$\mathrm{H}_{2}+h \nu \rightarrow \mathrm{H}^{+}+\mathrm{H}^{-}$ & 17.32 & 15.42 & {$[44,38,45,14,40]$} \\
$\mathrm{NO}+h \nu \rightarrow \mathrm{O}^{-}+\mathrm{N}^{+}$ & 19.54 & 14.01 & {$[38]$} \\
$\mathrm{CO}+h \nu \rightarrow \mathrm{C}^{+}+\mathrm{O}^{-}$ & 20.90 & 14.01 & {$[38]$} \\
$\mathrm{CO}+h \nu \rightarrow \mathrm{C}^{-}+\mathrm{O}^{+}$ & 23.44 & 12.89 & {$[46]$} \\
$\mathrm{N}_{2} \mathrm{O}+h \nu \rightarrow \mathrm{O}^{-}+\mathrm{N}_{2}^{+}$ & 15.79 & 11.40 & {$[47]$} \\
$\mathrm{HCCH}+h \nu \rightarrow \mathrm{H}^{+}+\mathrm{C}_{2} \mathrm{H}^{-}$ & 16.32 & 11.28 & {$[48,33,49]$} \\
$\mathrm{CH}_{3} \mathrm{Cl}+h \nu \rightarrow \mathrm{CH}_{3}^{+}+\mathrm{Cl}^{-}$ & 9.78 & & {$[38]$} \\
\hline \hline
\end{tabular}

Table 1.1: A Brief Survey of Experimental Photoion-pair Formation Studies. This table includes the three molecules studied in this thesis: $\mathrm{HCl}, \mathrm{O}_{2}$ and $\mathrm{HF}$. The molecular ionization potentials are also listed. A more comprehensive list of observed photoion-pair formation processes is available in Table I of Berkowitz's review [34]. 
spectrum of $\mathrm{O}_{2}$ photoion-pair formation in the vicinity of the energetic threshold, reproduced in Fig. 1.5. This spectrum consists of a series of "steps", separated in energy by the ground state rotational energy levels. These energy spacings - which are well-known from other techniques - have been superimposed on Fig. 1.5. Each step corresponds to an additional initial rotational state being allowed to dissociate into ion-pairs. The more initial rotation a molecule has, the less energy it takes to reach the ion-pair dissociation threshold; see Fig. 1.6.

The energetics for photolysis into neutrals should be similar, since dissociation asymptotes are independent of the relative rotation of the two departing fragments. However, for neutrals it is common for the centrifugal barrier to prevent observation of the true energetic threshold [4]. This barrier is due to the effect of the addition of the centrifugal contribution $V_{\text {rot }}(R)$, on the normal rotationless potential:

$$
V_{\text {rot }}(R)=\frac{J(J+1) \hbar^{2}}{2 \mu R^{2}}
$$

where $J$ is the rotational state, $R$ is the internuclear separation, and $\mu$ is the reduced mass of the two body system. Figure 1.7 illustrates the formation of a barrier due to this term. Because the long range potential of ion-pair states is much stronger than the long-range potentials for states which dissociate into neutrals, the barrier does not appear to form for ion-pair states. Although it may be proven that a barrier must form for potentials with weak $-1 / R^{6}$ tails, it is not possible to prove generally that longrange coulombic potentials cannot exhibit a barrier. For example, for the analogous photoionization problem, an electron moving in the effective potential of a nucleus and the surrounding electrons comprising an ion-core, can encounter a small barrier due to the centrifugal effect (see Fig. 8, on Pg. 49 of Berkowitz's book [53]). However, it is relatively straightforward to demonstrate that under reasonable assumptions, the longrange coulomb potential in ion-pair states, prevents formation of a barrier [4].

This situation is analogous to the relationship between photoionization and photodetachment, where photoionization cross-sections can be non-zero at threshold, whereas photodetachment cross-sections must always be zero at threshold [52]. 


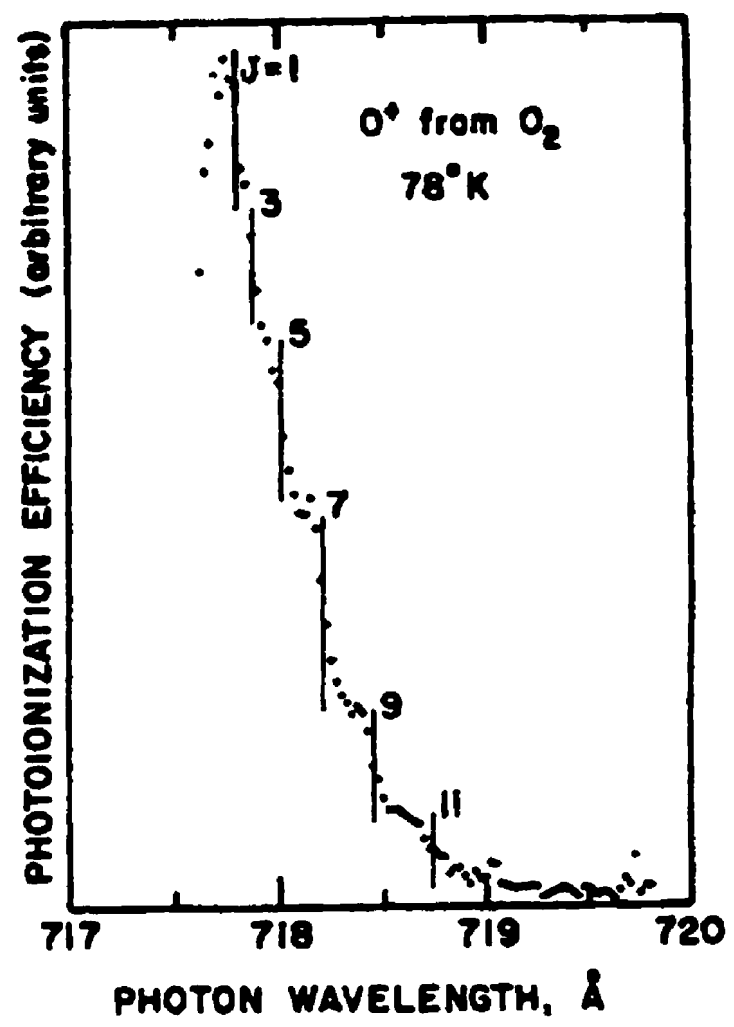

Figure 1.5: Threshold photoion-pair formation from $\mathrm{O}_{2}$. From Ref. [37]. The rotational energy level spacings of the ground vibronic state have been superimposed. 


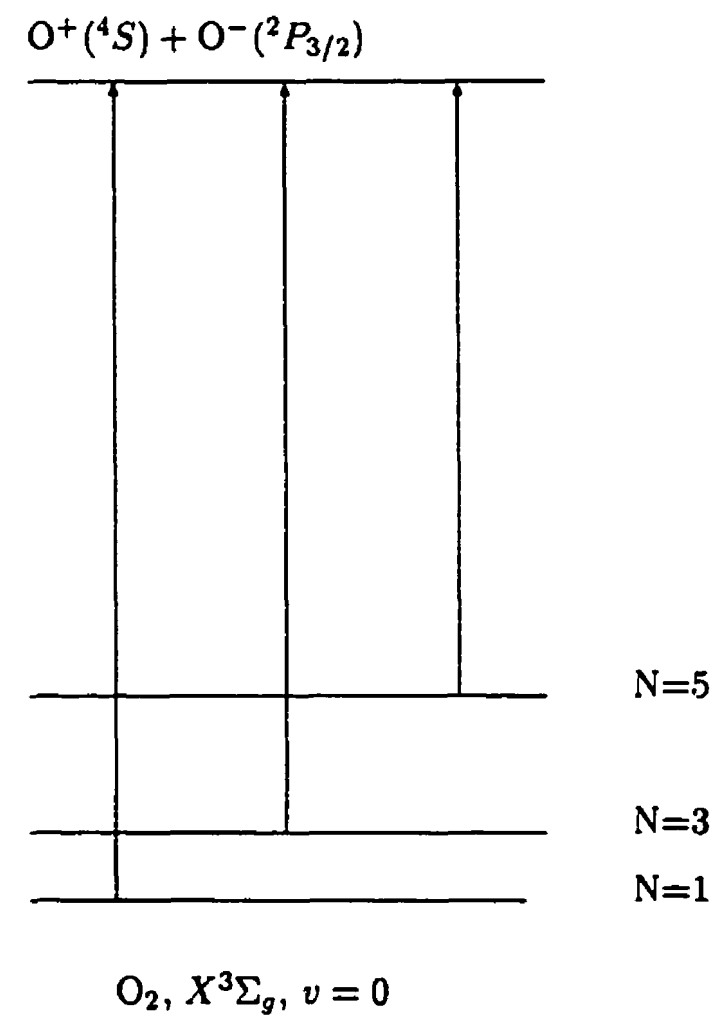

Figure 1.6: Energetics of Ion-Pair Formation in $\mathrm{O}_{2}$. This figure illustrates how the photon energy required to reach the ion-pair dissociation threshold in $\mathrm{O}_{2}$ decreases with additional initial rotational excitation. This rationalizes the spacing of the steps observed in Fig. 1.5. 
So, as discussed, neither Wigner's threshold laws, nor centrifugal barriers prevent observation of a photoion-pair signal directly at threshold. This is useful, as the crosssection for producing ion-pair states just above threshold is intimately related to the ease by which high- $v$ ion-pair states may be excited just below threshold - in analogy with the Rydberg-state case. It is reasonable to expect, and can be justified rigorously, that the concept of continuity of oscillator strength - previously discussed in the context of Rydberg states and photoionization - also applies to photodissociation [54]. Specifically, the probability of exciting unresolved high-v ion-pair states below an ion-pair formation threshold is exactly the same as producing ion-pairs just above this threshold. Since numerous photoion-pair yield spectra cover threshold regions, this connection allows one to establish how feasible excitation of high-v ion-pair states will be. Although useful as a rough guide, actual identification of the ion-pair threshold region can be problematic. Photoion-pair yield spectra do not typically look like Fig. 1.5 at threshold. Identification of a step-like structure can be difficult, because as soon as an ion-pair channel opens, its cross-section can vary dramatically with energy, showing strong resonances, obscuring identification of the higher energy steps. This is a consequence of the indirect mechanism for producing ion-pairs discussed earlier.

\subsubsection{Threshold Photoion-pair Production Spectroscopy and the De- termination of Dissociation Energies}

The previous section discussed the excitation of high-v ion-pair states, and the relationship with photoion-pair formation at threshold. Given that excitation to high- $v$ ion-pair states is practical, could this be detected? Based on the discussion in Section 1.3, it is expected that electric fields may field-dissociate high-v ion-pair states. One possibility is to apply a weak field-dissociating pulse immediately following photoexcitation. However, this would not allow discrimination between the field-dissociation signal and the prompt photoion-pair signal. A spectrum taken by scanning photon-energy and collecting ion- 


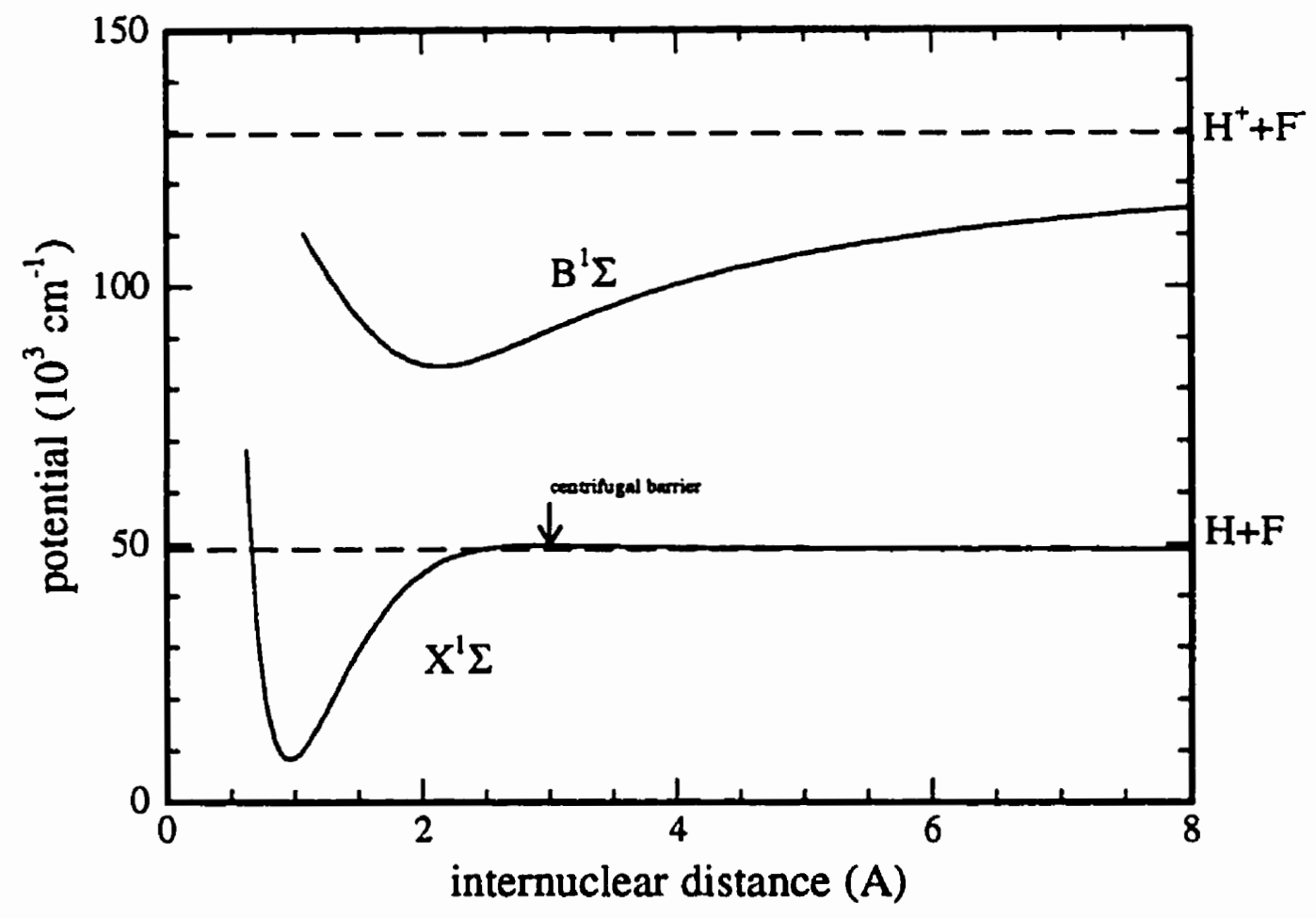

Figure 1.7: Formation of a centrifugal barrier. This diagram illustrates the same RKR derived potential energy curves for HF as in Fig. 1.1, but with the centrifugal contribution, added on for $J=20$ (see Eqn. 1.24). The $X^{1} \Sigma$ ground state exhibits formation of a barrier to dissociation, while the ion-pair state $B^{1} \Sigma$ does not. (A large $J$ has been chosen to make the barrier observable on the scale of the figure. For lower $J$, smaller barriers form.) 
signal immediately following an electric field pulse would appear similar to that of Fig. 1.5 , only with the step-like structure shifted to lower energy due to the field dissociation contribution.

Although a single pulse is not sufficient to selectively detect excitation to high-v ion-pair states, it can be envisioned that a combination of two electric field pulses may work. The first would discriminate against the prompt ions, pushing them out of the field of view, and the second would field-dissociate the high-v ion-pair states - if they had been excited. By scanning photon energy and monitoring the ion-signal due to this second pulse, it should be possible to collect spectra which are sensitive to excitation of a particular range of high-v ion-pair states below each threshold. Because of practical limitations regarding the sizes of electric-fields, and excitation bandwidth, it would not be expected that individual ion-pair states could be resolved. A single peak would be observed for each ion-pair threshold, corresponding to many unresolved high- $v$ ion-pair states. For example, if the threshold region for photoion-pair formation of $\mathrm{O}_{2}$ were scanned (see Fig. 1.5), one would expect to see peaks for each of the different initial $J$ states, at energies just below the field-free thresholds.

By varying the magnitudes of both the discrimination field pulse and field-dissociating pulse, it should be possible to change the shape of the peaks. The discrimination field, besides ridding the area of prompt ions, also has the effect of depleting the highest- $v$ ionpair states. Thus if its magnitude is increased, one would expect that the peaks would have their higher energy - or blue edges - depleted. By collecting spectra with different discrimination fields, and observing the shifts in the blue edges of these peaks, it should be possible to extrapolate to zero-field strength. At zero-field strength, the blue-edges should coincide with the field-free ion-pair formation thresholds - which are difficult to determine from the photoion-yield spectra alone.

It has been possible to apply this technique - Threshold Ion-Pair Production Spectroscopy (TIPPS) - to the $\mathrm{HF}, \mathrm{HCl}$ and $\mathrm{O}_{2}$ molecules $[41,36]$. The scheme described here, 
for discriminating against prompt ions, and detecting those produced by a subsequent pulse is both practically and conceptually similar to Mass-Analyzed Threshold Ionization spectroscopy (MATI) $[29,55]$. In fact, in Chapter 3 it is illustrated how the spectrometer used to study the electric field induced dissociation of high- $v$ ion-pair states may also perform MATI spectroscopy of argon, and determine its field-free ionization threshold.

Besides demonstrating that high-v ion-pair states may be excited and field-dissociated, are there any other possible applications of the TIPPS technique? It has been mentioned how it was expected that a systematic variation of the discrimination field magnitude could allow one to determine the field-free ion-pair formation thresholds. Measurement of an energetic threshold for splitting a diatomic molecule $\mathrm{AB}$ into ion-pairs $E_{\mathrm{A}^{+}, \mathrm{B}^{-}}$, allows determination of the dissociation energy of the molecule $D_{0}(A B)$, if the electron affinity $(E A(B))$ and ionization potential $I P(A)$ of the fragments are known: ${ }^{12}$

$$
D_{0}(\mathrm{AB})=E_{\mathrm{A}^{+}, \mathrm{B}^{-}}-I P(\mathrm{~A})+E A(\mathrm{~B}) .
$$

Almost all atomic ionization potentials are known to accuracies better than $1 \mathrm{~cm}^{-1}$, as are many electron affinities [52].

There have been a variety of methods applied to the determination of molecular dissociation energies. One method is to monitor photofragment yield of the molecule while scanning incident photon energy - determining the energetic threshold for dissociation from the minimum photon energy at which the fragments appear. Unfortunately, thermal excitation of the sample, resonances above threshold, and poor Franck-Condon factors can make this difficult. An alternative approach is to use the spectroscopically determined bound state energy levels of a molecule near a dissociation threshold. For diatomic molecules this involves the Birge-Sponer extrapolation $[4,57]$, or suggested extensions [58]. However, these rely on the existence of extensive unperturbed spectroscopic data near the dissociation limit, and their application is not always straightforward [51], nor

\footnotetext{
${ }^{12}$ For examples of usage of Eqn. 1.25 in determining bond energies, see the review by Berkowitz et al. [56].
} 
easily extendible to triatomic and larger molecules.

On the other hand, there is nothing to restrict application of the TIPPS technique to the determination of diatomic dissociation energies. For example, the process:

$$
\mathrm{CH}_{3} \mathrm{Cl}+h \nu \rightarrow \mathrm{CH}_{3}^{+}+\mathrm{Cl}^{-} \text {, }
$$

has been observed $[48,33,49]$. Since $I P\left(\mathrm{CH}_{3}\right)$ is known to $\pm 3 \mathrm{~cm}^{-1}[59]$, and $E A(\mathrm{Cl})$ is known to $\pm 0.5 \mathrm{~cm}^{-1}[60]$, determination of the threshold for ion-pair formation would establish the $\mathrm{C}-\mathrm{Cl}$ bond strength in $\mathrm{CH}_{3} \mathrm{Cl}$ with unprecedented accuracy. Current estimates of this bond energy are accurate to no better than $100 \mathrm{~cm}^{-1}$. Of course, not all molecules have suitable photoion-pair processes. However, based on the previous photoion-pair studies, reviewed by Berkowitz [34], the number of potential targets is large.

Unfortunately, time did not permit the study of a polyatomic. However, each of the three diatomics studied, illustrates interesting aspects of the TIPPS technique. The chapters devoted to $\mathrm{HCl}$ and $\mathrm{HF}$ illustrate the determination of dissociation energies as outlined above. In the case of $\mathrm{HCl}$, the new dissociation energy obtained has a substantially improved accuracy compared to the previously known thermochemical value. For HF, the individual TIPPS lines exhibit complex structure, reflecting the predissociation mechanism responsible for production of the high- $v$ ion-pair states. TIPPS studies of the $\mathrm{O}_{2}$ molecule show a second set of ion-pair formation thresholds, corresponding to the production high- $v$ ion-pair states, where the $\mathrm{O}^{-}$partner is an excited spin-orbit state. Before discussing these results, it is necessary to describe the experimental techniques used. This is the subject of the following chapter. 


\section{Chapter 2}

\section{Experimental}

\subsection{Introduction}

The experiments described in this thesis were carried out using a modified version of the apparatus described by Kong et al.[61, 62]. This apparatus consists of three major subsystems:

1. Vacuum ultraviolet (VUV) generation system - to excite the molecules from their ground states to high- $n$ Rydberg states, or to high- $v$ ion-pair states,

2. Pulsed Molecular beam - to introduce the sample gas into a collision-free environment, and

3. Spectrometer and detection electronics - to detect excitation into the high- $v$ ion-pair states by electric-field induced dissociation.

This chapter will discuss each of these components of the experimental apparatus. 


\subsection{Generation of Vacuum Ultraviolet Radiation}

\subsubsection{Introduction}

Examination of Table 1.1, shows that the energetic thresholds for single-photon ion-pair formation for many molecules are high. For example, the molecules studied in this thesis - $\mathrm{HCl}, \mathrm{O}_{2}$, and $\mathrm{HF}$ - have energetic thresholds at $14.4 \mathrm{eV}(85.6 \mathrm{~nm}), 17.3 \mathrm{eV}(71.8$ $\mathrm{nm})$ and $16.1 \mathrm{eV}(77.2 \mathrm{~nm})$, respectively. These energies are in the range known as the vacuum ultraviolet (VUV), which covers from where $\mathrm{O}_{2}$ atmospheric absorption becomes a problem, to the soft $x$-ray regime $(190 \mathrm{~nm}-10 \mathrm{~nm}, 6.5 \mathrm{eV}-120 \mathrm{eV})$. Generation of light in this spectral range requires specialized techniques. In particular, at wavelengths below $105 \mathrm{~nm}(11.8 \mathrm{eV})$, it becomes necessary to work "windowless", since no materials of structural thickness are transparent [63]. ${ }^{1}$

Photoion-pair formation has been studied with three different sources of VUV radiation:

1. Monochromatized radiation from a lamp (e.g. Dehmer and Chupka [37]),

2. Monochromatized synchrotron radiation (e.g. Yencha and co-workers $[35,64]$ ), and

3. Nonlinear frequency mixing of laser radiation (e.g. Munakata and Kasuya [33]).

Each of these sources has advantages and disadvantages. Both monochromatized sources (lamp and synchrotron) are broadly tunable - large energy ranges may be covered quickly for survey work. Generally speaking synchrotron radiation is the brighter of the two, but requires use of an elaborate facility, whereas lamps and lasers can be operated in small laboratories.

For these experiments, nonlinear frequency mixing of dye laser radiation was used to generate the required VUV. This source has the advantage of high spectral brightness

\footnotetext{
'Some workers use the term "extreme-ultraviolet" (XUV or EUV), to refer to this "windowless" region below $105 \mathrm{~nm}$.
} 
(photons per unit bandwidth) ${ }^{2}$ and a time structure suitable for the TIPPS experiments. In particular, light arrives every $100 \mathrm{~ms}$ in short pulses ( $<5 \mathrm{~ns})$. This delay between pulses allows sufficient time for discrimination against prompt ions, which is a problem with cw-sources (lamps), and pseudo cw-sources (synchrotrons).

For further information on VUV sources using nonlinear mixing, especially for their application in Chemical Physics, the reader is referred to the series of review articles by Hepburn $[66,67,68]$. The book by Hanna et al. [69] is useful but limited to work prior to 1979. The review of Hilbig et al. [70], and the thesis of Lago [71] are recommended for the specific techniques used in this work. Because of the excellent background material already available, the theory of nonlinear mixing will only briefly be touched upon.

\subsubsection{Theory}

The term "nonlinear" refers to the response of the bulk polarization $P$ of a material to an applied electric field $E$ (considering components in one direction only, for simplicity):

$$
P=\chi^{(1)} E+\chi^{(2)} E^{2}+\ldots
$$

The higher-order nonlinear terms $\chi^{(2)} E^{2}, \chi^{(3)} E^{3}$ are responsible for the generation of harmonics. For instance, if a field of $E=E_{0} \sin (\omega t)$ is applied to the material, $P$ will contain an oscillatory component at $\omega$, due to the $\chi^{(1)} E$ term, but also at $2 \omega$, due to $\chi^{(2)} E^{2}$, since $\sin ^{2}(\omega t)=\frac{1-\cos (2 \omega t)}{2}$. This acts as a source for new radiation at $2 \omega-$ the "second harmonic". Second harmonic generation was first observed shortly following the invention of the laser [72]. Since then both second and higher order effects have become a powerful tool for extending the wavelength coverage of lasers.

With harmonic generation it is necessary to use a medium for which absorption of the generated beam will be minimal. This rules out the use of solid or liquid materials for generation of VUV below $105 \mathrm{~nm}$. Gases must be used. But there is no second harmonic

\footnotetext{
${ }^{2}$ A comparison of the spectral brightness of laser and synchrotron sources is given by Hinnen [65].
} 
generation in gases, since the $\chi^{(2)}$ term must be zero for isotropic media (along with all other $\chi^{(n)}, n$ even terms). Thus the first non-linear frequency conversion process allowed in a gas is third-harmonic generation (due to $\chi^{(3)}$ ).

Although third harmonic generation is commonly used to generate tunable VUV, it is useful to introduce a slight modification to enhance conversion efficiency. Thus far, the frequency dependencies of the $\chi$ coefficients have been neglected. The $\chi$ 's show resonances. In particular, by introducing two laser beams $\left(\nu_{1}\right.$ and $\left.\nu_{2}\right)$ into the media, with one tuned to a two-photon resonance $\left(\nu_{1}\right)$, it is possible to greatly enhance the third-order mixing process for the generation of $2 \nu_{1}+\nu_{2}$. The $\nu_{1}$ frequency remains fixed to the resonance, while $\nu_{2}$ may be tuned to vary the frequency of the generated VUV. A monochromator may then be used to pick out the desired harmonic from the residual fundamentals and other harmonics generated $\left(3 \nu_{1}, 2 \nu_{1}-\nu_{2}\right)$. This technique is known as "resonantly-enhanced four-wave sum-frequency mixing".

Resonantly-enhanced four-wave sum-frequency mixing was demonstrated in atomic vapours as early as $1974[73,74,75]$. However, this process became much more practical with the use of the noble gases. These may easily be introduced to the mixing region using a pulsed valve, reducing average gas load, while allowing high densities for good conversion efficiency $[76,77]$. Development of the BBO material in the late eighties [78], enabled visible dye laser radiation to be converted to wavelengths as low as $190 \mathrm{~nm}$, allowing many $\mathrm{Xe}$ and $\mathrm{Kr}$ two-photon resonances to be covered.

\subsubsection{Practice}

The source of VUV radiation for these experiments was resonantly enhanced four-wave sum-frequency mixing in a pulsed jet of $\mathrm{Xe}$ or $\mathrm{Kr}$. The two dye laser beams required for the mixing process are overlapped and focused with a lens $(f=20 \mathrm{~cm})$ into a vacuum chamber. Before focusing, the beam waists are on the order of 1-2 $\mathrm{mm}$. The focal spots of the beams are as close as possible to the nozzle of a pulsed valve, which is emitting pulses of 
$\mathrm{Xe}$ or $\mathrm{Kr}$ at the laser repetition rate. The generated harmonics and fundamentals emerge from the jet and are separated and refocused by a $1 \mathrm{~m}$ vacuum monochromator. After exiting the monochromator, the VUV beam enters the main chamber, passes through the molecular beam and spectrometer, and then is detected by a dual microchannel plate detector. This detector is normally operated with very low electron gain $(\approx 10-100)$, but is very useful for initially finding and optimizing the VUV signal, when it is necessary to go to much higher gain to see weak signals.

The VUV generation system was built and used for the thesis work of Kong [61, 62]. Since then, the pumping system has been modified slightly. The monochromator chamber was originally pumped by a $1500 \mathrm{~L} / \mathrm{s}$ cryopump. This was replaced with a $260 \mathrm{~L} / \mathrm{s}$ turbopump. A small chamber separates the mixing chamber from the monochromator and this is pumped with a small $\left(6^{\prime \prime} \emptyset\right)$ oil diffusion pump. By introducing this extra stage of differential pumping, the gas load on the monochromator remains light, allowing low pressures to be acheived ( $10^{-6}$ torr), thereby preventing reabsorption of the generated VUV. $^{3}$ The main experimental chamber is also differentially pumped, using a $1000 \mathrm{~L} / \mathrm{s}$ turbopump.

It is useful to be able to determine how much VUV is made in this setup. Since it is difficult to absolutely calibrate the microchannel plate detector - which is normally used to monitor the VUV - a simple photodiode was used (see for example Ref.'s [79] and [65]). Photoelectrons emitted from a copper surface were collected, and the total charge was measured by integrating the voltage pulse generated over a $50 \Omega$ resistor (see, for example, Fig. 2.4 of Ref. [65]). The quantum efficiency of photoelectron production by copper is available [63], allowing one to convert the measured photoelectron yield into a VUV photon fluence. A typical value obtained was $5 \times 10^{7}$ photons/pulse.

The spot size of the generated VUV is important for considering the possible perturbations due to neighbouring ions. Lago and co-workers $[80,71]$ have considered how

\footnotetext{
${ }^{3}$ The generated VUV photon energy is normally above the ionization potential of the mixing gas, leading to broadband absorption by ionization.
} 
the focusing of the input beams determines the properties of the generated harmonics (especially the confocal parameter). But it is difficult to test these predictions, since the generated harmonics normally remain "buried" in the fundamentals, until separation by a monochromator. Naturally, the monochromator has its own effect on the beam properties. In particular, one expects astigmatic refocusing, in addition to the normal diffractive effects. While it is possible to model this, many assumptions have to be made; thus the only really satisfactory solution is to actually measure the VUV spot size. Unfortunately, because of time restrictions this was not done. One possible approach would be to use a microchannel plate followed by a phosphor read-out (similar to image intensifier technology). To obtain rough estimates of certain properties, the spot-size has been estimated to be a rectangular patch of $1000 \mu \mathrm{m}$ (V) by $150 \mu \mathrm{m}(\mathrm{H})$.

\subsubsection{Dye Lasers}

The harmonics of a Nd:YAG laser were used to pump the two tunable dye lasers used for four-wave mixing. The Nd:YAG laser (Spectra Physics [81], GCR-4) was Q-switched [82] to produce short $(\approx 8 \mathrm{~ns})$, intense $\left(\approx 1000 \mathrm{~mJ} /\right.$ pulse) pulses in the near-infrared $\left(\lambda_{Y A G}\right.$ $=1.064 \mu \mathrm{m}$ ) with a repetition rate of $10 \mathrm{~Hz}$. This radiation was frequency doubled in a non-linear crystal to produce green light $\left(\lambda_{Y A G} / 2=532 \mathrm{~nm}\right)$. The green light could be used to pump a dye laser and/or sum-mixed with the residual infra-red to generate ultraviolet light $\left(\lambda_{Y A G} / 3=355 \mathrm{~nm}\right)$. The ultraviolet light could then be used to pump either one or both of the dye lasers. Laser dyes suitable for operation below $545 \mathrm{~nm}$ must be pumped with the $355 \mathrm{~nm}$ light ("blue-pumping"). However, at wavelengths greater than $545 \mathrm{~nm}$, pumping with $532 \mathrm{~nm}$ is normally used ("green-pumping"), since it is usually more efficient than blue-pumping. With minor changes to the setup it was possible to switch between all possible pumping configurations (blue-blue, blue-green, and green-green pumping), and obtain the maximum recommended pump energy for each laser $(\approx 100 \mathrm{~mJ} /$ pulse $)$. 
The two pulsed dye lasers (both Lambda Physik [83], FL3002), are of the Hänsch type [84]. The oscillator cavity consists of an end mirror, a cuvette with a flowing dye solution, and a 600 lines/mm grating in the Littrow configuration to provide wavelength selective feedback in the cavity. Within the gain-band of a given dye-solvent combination, tunability is obtained by rotating the angle between the grating normal and cavity axis $(\theta)$. The limited gain bandwidth of the dye restricts oscillation to one particular order $(n)$. Thus, the lasing wavelength $(\lambda)$ is given by the "grating equation" for the Littrow configuration:

$$
n \lambda=2 d \sin (\theta)
$$

where $d$ is the groove spacing (in this case, $1 / 600 \mathrm{~mm}$ ). A microprocessor controlled stepper motor controls $\sin \theta$, and a counter number "labels" these discrete positions. A wavelength scan consists of stepping sequentially through these positions. Since the dye laser frequencies are related to the final VUV frequency in simple manner, ${ }^{4}$ determination of the final VUV frequency is a matter of relating the counter position to dye laser frequency. Dye laser calibrations were based on the observation of known transitions of $U$ and $\mathrm{Ne}$, and etalon frequency markers were used to interpolate between these transitions. These matters are discussed in Appendix A.

After being coupled out of the oscillator cavity, the laser beam passes through two consecutive single-pass dye amplifiers (these are part of the FL3002 units). After the final amplification stage, the visible frequency may be doubled in a nonlinear crystal to extend the visible dye laser output into the ultraviolet. Either Potassium Dihydrogen Phosphate (KDP) or Beta-Barium Borate (BBO) crystals are used, depending on the wavelength. A comprehensive survey of the properties of these and other nonlinear crystals is available [87]. It was also necessary to generate ultraviolet radiation below the frequency-doubling limit of BBO (<205 nm). In this case, frequency doubled light $(\approx 300 \mathrm{~nm})$ was sum-

\footnotetext{
'It is assumed that the relationship $v_{v} v=2 \nu_{1}+\nu_{2}$ holds exactly, and also that the second harmonics of the dye laser radiation are exactly twice the frequency of the fundamentals. For higher resolution work the breakdown of these relationships must be considered (see Ref.'s [85] and [86]).
} 
mixed with the residual fundamental $(\approx 600 \mathrm{~nm})$ to produce very short wavelength UV $(\approx 200 \mathrm{~nm}$ ). The particular dyes and crystals used for each individual experiment are summarized throughout the thesis.

It is necessary to overlap the two beams both spatially and temporally for the fourwave mixing process. The beams from both dye lasers were combined on an optical table using a single dichroic mirror. These mirrors are coated to allow reflection of a small range of wavelengths, and low-loss transmission of other wavelengths, enabling the overlap of two beams of different frequency. Since $\nu_{1}$ is normally fixed (to a two-photon resonance), it is useful to make this the reflected wavelength. Dichroics were available for the different two-photon resonances in $\mathrm{Xe}$ and $\mathrm{Kr}$.

Care is taken to synchronize the arrival of the $\nu_{1}$ and $\nu_{2}$ beams at the gas jet to within $1 \mathrm{~ns}$, as the beams must interact with each other for frequency mixing.

\subsection{Molecular Beam}

In the experiments described in this thesis, the sample gas was introduced to the vacuum chamber using a pulsed supersonic molecular beam. There are several advantages to this technique, which is discussed thoroughly in a book edited by Scoles [88], and a recent review article by Morse [89].

Since both highly vibrationally excited ion-pair and high- $n$ Rydberg states are fragile, it is desirable to perform experiments on them at low densities and temperatures, so that the influence of surrounding neutrals and ions can be minimized. In a low-density environment, the number of molecules excited is few, so the ions resulting from field ionization/dissociation must be detected with high gain. Due to the problems of ionfeedback, high gain detectors such as channeltrons and microchannel plates must be used at low pressures. Also, it is desirable to avoid ion-molecule reactions when transporting the ions from their point of origin to the detector. This explains the usefulness of the 
pulsed beam technique: the sample may be introduced in a cold "beam" which crosses the light, presenting a higher density in the interaction region, while preserving the lowdensities required for good detection. The $100 \mathrm{~ms}$ between light pulses can then be used to pump away the sample gas before the next shot.

In these experiments, the source of the beam was a solenoid valve (General Valve, Series 9), which lifts a small Teflon poppet off a $1 \mathrm{~mm}$ orifice, allowing the sample gas to flow from the high pressure (1-2 atm) reservoir into the chamber. When the gas molecules rush out into the low-pressure environment, they undergo an isentropic expansion (no heat flow). Their thermal energy is converted into a directed flow, to speeds which exceed the local speed of sound, prompting the designation "supersonic". After a certain point in the expansion, the molecules effectively stop colliding, as their density and temperature are greatly reduced. Experiments are usually performed on the sample in this region.

The average speed that the molecules finally reach, $v_{\infty}$, may be calculated from Eqn. 2.2 of Miller [90]:

$$
v_{\infty}=\sqrt{\frac{2 k}{m}\left(\frac{\gamma}{\gamma-1}\right) T_{0}}
$$

where $\gamma=c_{p} / c_{v}$ (=5/3 for monatomic gases), $k$ is Boltzmann's constant $\left(1.38 \times 10^{-23}\right.$ $\mathrm{J} / \mathrm{K}), m$ is the weight of the species, and $T_{0}$ is the temperature of the reservoir. Using this equation, it is found that room temperature argon ${ }^{5}$ will reach a terminal speed of $560 \mathrm{~m} / \mathrm{s}$. An important parameter is the velocity distribution about this mean speed, as it is needed to estimate collision rates in the beam. This is difficult to compute. ${ }^{6}$ The

\footnotetext{
${ }^{3}$ To make things specific, all calculations in this section consider Ar. The results are not too much different for other species.

'Experimental and theoretical predictions of the velocity distribution are available for continuous sources. Unfortunately, these can be difficult to apply to pulsed sources. In particular, in this case, the "effective" nozzle diameter is typically much smaller than the real diameter, due to incomplete opening of the valve. For example, if Eqn. 2.19b in Ref. [90] is used to compute the continuous nozzle flow rate, the resulting value is too high, by about 2 orders of magnitude (compared to the estimate given in the next paragraph).
} 
distribution may be roughly characterized by an effective temperature. For the conditions of our experiment, this should be less than $1 \mathrm{~K}$.

Perhaps the most important property of the molecular beam is its density in the region of interaction with the light beam. Miller [90], has summarized the results of Beijerinck and Verster [91]. They find that the centerline particle flux (for a monatomic gas, such as $\mathrm{Ar}$ ) is given by:

$$
F=(\text { molecules } /(\text { unit time and area }))=2 \frac{\dot{N}}{\pi r^{2}}
$$

where $\dot{N}$ is the nozzle flow rate (total number of molecules emerging per unit time), and $r$ is the distance from the nozzle opening. The centerline particle flux may then be divided by $v_{\infty}$, to obtain an estimate for the number density, $n$, in the interaction region:

$$
n=\frac{2 \dot{N}}{v_{\infty} \pi r^{2}} .
$$

The nozzle flow rate, $\dot{N}$, may be estimated in the following manner. While the beam is on, the average pressure in the main chamber is $10^{-5}$ torr. This chamber is being pumped by a $1000 \mathrm{~L} / \mathrm{s}$ pump. So the average gas load on the chamber is $10^{-2}$ torr $\mathrm{L} / \mathrm{s}$. Since gas is emitted 10 times a second, the gas in a single pulse is $10^{-3}$ torr $\mathrm{L}$. Assuming that the gas is emitted in $500 \mu$ sulses, the average nozzle flow rate during a pulse is: $\dot{N}=6.4 \times 10^{19} \mathrm{~s}^{-1}$.

The nozzle was located $5.2 \mathrm{~cm}$ away from the VUV beam axis in all of the experiments. So, substituting $r=5.2 \mathrm{~cm}, v_{\infty}=560 \mathrm{~m} / \mathrm{s}$, and $\dot{N}=6.4 \times 10^{19} \mathrm{~s}^{-1}$ into Eqn. 2.4 , an estimate of $n=3 \times 10^{13} \mathrm{~cm}^{-3}$ is obtained.

With the estimates of temperature and density in the beam, it is possible to estimate the effect of collisions, during the waiting period between excitation and fieldionization/extraction. Recall the discussion of the sizes of Rydberg and ion-pair states given in Section 1.1. For $1 \mathrm{~cm}^{-1}$ below threshold, $\langle r\rangle=8.7 \times 10^{-4} \mathrm{~cm}$. Thus at a number density of $3 \times 10^{13} \mathrm{~cm}^{-3}$, there is an excellent chance that a neutral will be located within an ion-pair or Rydberg state. However, not too much harm is expected 
from this. The collisions between the individual partners of the Rydberg or ion-pair states, and the neutrals, are more significant. We are concerned with collision rates on the order of $(1 \mu \mathrm{s})^{-1}$, as these are significant on the time-scale between excitation and field-ionization/dissociation, in both MATI and TIPPS. The collision rate $(Z)$ of one molecule is given by:

$$
Z=\sqrt{2} n \sigma\left(\frac{8 k T}{\pi m}\right)^{1 / 2}
$$

where $\sigma$ is the cross-section for the process being considered. Substituting $Z=1 \mu \mathrm{s}^{-1}$, $T=1 \mathrm{~K}$ and $n=3 \times 10^{13} \mathrm{~cm}^{-3}$, and rearranging, it is found that $\sigma>1 \times 10^{-11} \mathrm{~cm}^{2}$. could cause problems. Since ion-neutral cross-sections are typically much smaller than this, collisions with neutrals are not expected to be significant.

To consider the effects of collisions with surrounding ions, it is necessary to know the ion-density. This may be computed from the neutral density, if we know the probability of ionization. For low probabilities of excitation (as in this case), this is simply the ionization cross-section times the photon fluence for the region (in photons/unit area). Assuming $10^{8}$ photons arrive in a $1000 \mu \mathrm{m} \times 150 \mu \mathrm{m}$ spot, and an ionization cross-section of $10 \mathrm{Mb}$, the probability of ionization is $6.6 \times 10^{-7}$. With a neutral density of $n=3 \times 10^{13} \mathrm{~cm}^{-3}$, the ion-density after passage of the light will be $2 \times 10^{7} \mathrm{~cm}^{-3}$. Although the number density of ions is significantly lower than that of the neutrals, their collision cross-sections are generally much higher. Simply rescaling the estimate for neutral collisions given above, cross-sections above $1.5 \times 10^{-5} \mathrm{~cm}^{2}$, will present problems. This is approximately the geometrical size of a Rydberg or ion-pair state with a binding energy of $0.4 \mathrm{~cm}^{-1}$.

Collision cross-sections between ions and Rydberg or ion-pair states may be so high, that under certain conditions, we may think of a collision complex being formed by photoexcitation. Before photoexcitation, a neutral and ion are far enough away from one another that they are not interacting; after excitation, the Rydberg/ion-pair state and neutral are interacting. This is the interpretation of the rapid $\ell$ and $m_{\ell}$ mixing which is hypothesized to be responsible for Rydberg-state stabilization against autoionization 
[92]. The Rydberg states are excited in the presence of nearby ions, which break the normal spherical and cylindrical symmetry.

As shall be seen, the effects of ion-density on both Rydberg and ion-pair states with low binding energies are observable in MATI and TIPPS spectra.

\subsection{Spectrometer}

\subsubsection{Introduction}

A spectrometer was constructed for the experiments described in this thesis. As outlined previously, the purpose of a spectrometer for MATI or TIPPS experiments is to differentiate between the ions formed during photoexcitation - the so-called prompt ions - and those formed by the field-dissociating (TIPPS) or field-ionizing (MATI) pulse. Johnson and $\mathrm{Zhu}$ [55] discuss a variety of ways that this may be accomplished. The scheme illustrated in Fig. 2.1 was chosen because of its simplicity. It is not considered optimal in any way - other than perhaps for its ease of construction. Further studies should investigate the use of spectrometers described in the literature (see, for example, Ref. [93]), which were originally used for MATI, but should be equally applicable to TIPPS. In general these use smaller electric fields for field-ionization, and thus are capable of much higher spectral resolution than the scheme described here.

\subsubsection{The Kinematics of Discrimination}

As Fig. 2.1 illustrates, prompt ions are accelerated into the region between P1 and P2 before the field-ionizing/extraction pulse is applied (a single pulse serves to both fieldionize or field-dissociate and extract). The desired fragments (if present) are then drawn up the time-of-flight (TOF) tube where they are detected, whereas the prompt ions between $\mathrm{P} 1$ and $\mathrm{P} 2$ are ignored. 


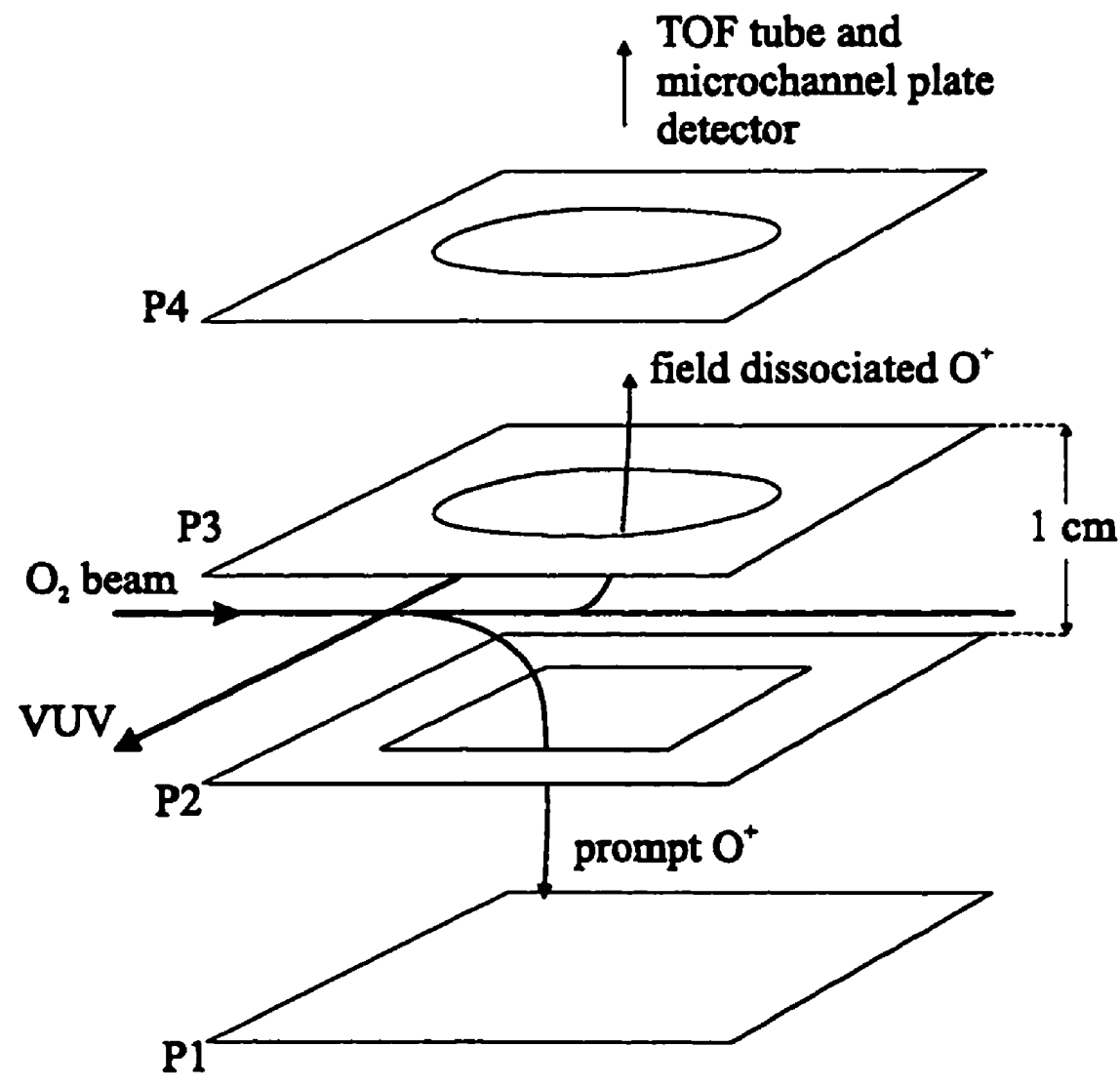

Figure 2.1: Schematic illustration of the discrimination capability of the spectrometer for the $\mathrm{O}_{2}$ TIPPS experiment. Prompt ions $\left(\mathrm{O}^{+}\right)$formed during photoexcitation are forced downwards by the discrimination field. After they are pushed below P2 they are drawn towards $P 1$ and remain undetected. Neutral states continue to travel in the direction of the beam after photoexcitation, and after all of the prompt $\mathrm{O}^{+}$ions have been drawn into the region between $\mathrm{P} 1$ and $\mathrm{P} 2$, an extraction and field-dissociating pulse is applied in the region between P2 and P3. This region then acts as the first acceleration stage of a Wiley-McLaren time-of-flight spectrometer, enabling the electric field dissociated $\mathrm{O}^{+}$ fragments to be discriminated against other ions $\left(\mathrm{O}_{2}^{+}\right)$by their time of flight (TOF) to a microchannel plate detector. 
It is possible to estimate the size of, and time for which the "discrimination" field must be applied to the region between $\mathrm{P} 2$ and $\mathrm{P} 3$, to ensure that the prompt ions reach $\mathrm{P} 2$, and subsequently travel into the P1-P2 region. As shown in Fig. 2.1, the molecular beam direction is orthogonal to the spectrometer axis. To a first approximation, the prompt ions have no velocity component along the spectrometer axis, and thus the distance that they travel along this axis, after the discrimination field $F_{d}$ is applied for a time $t$ is:

$$
d=\frac{1}{2} \frac{q_{e} F_{d}}{m} t^{2}
$$

where $m$ is the mass of the ion and $q_{e}$ is the elementary charge. Excitation occurs midway between $\mathrm{P} 1$ and $\mathrm{P} 2$, thus $d=0.5 \mathrm{~cm}$ is substituted into Eqn. 2.6, to get the discrimination criteria:

$$
\frac{F_{d} t_{d}^{2}}{m} \geq 1.04 \frac{\mathrm{V}}{\mathrm{cm}}(\mu \mathrm{s})^{2} \frac{1}{\mathrm{amu}}
$$

Eqn. 2.7 has been written as an inequality, as it represents a lower bound on $F_{d} t_{d}^{2} / m$, because:

1. The VUV spot is fairly broad (see Section 2.2.3), so excitation occurs over a range of $d$.

2. There will be some component of molecular beam velocity along the spectrometer axis, especially off the centerline axis of the molecular beam. This depends on the quality of alignment, and the VUV spot size.

3. When the ion-pairs are formed at energies above the threshold for ion-pair formation, the excess energy will be deposited into ion kinetic energy. Some fraction of ions will then have velocity components aiming away from P2, towards P3. These will take longer to reach P2 than those which are at rest initially. In this respect, discrimination in TIPPS spectroscopy differs from that in MATI, since for photoionization, the bulk of the excess energy is carried off by the electron, and the ion recoil energy is correspondingly small. 
4. Once reaching $P 2$, the ions will encounter a nickel mesh grid which they must pass through before entering the P1-P2 region. It is expected that a certain fraction of ions will "bounce" off this grid, back into the P2-P3 region, slow down and be re-accelerated back for another try. The quantitative details of the phenomenon are not known, however it will tend to increase the time required for discrimination.

In practice, because of the difficulty in estimating the combined effect of these factors, an empirical approach was taken; $F_{d}$ or $t_{d}$ were increased until adequate discrimination was obtained, using Eqn. 2.7 as a guide to the minimum $F_{d} t_{d}^{2} / m$ required. The actual $F_{d}$ 's and $t_{d}$ 's used are noted throughout the thesis.

\subsubsection{Field-Ionization and Extraction}

Once discrimination has taken place, a field-ionizing pulse is applied to the region between P2 and P3. This field also serves as an extraction pulse, drawing the ions up into the TOF spectrometer. The highly vibrationally excited ion-pair states travel along with the molecular beam after excitation, between the plates P2 and P3. Since discrimination times can be as large as $8.4 \mu \mathrm{s}$, the distance travelled in this period can be quite large ( $8.4 \mathrm{~mm}$ for a $1000 \mathrm{~m} / \mathrm{s}$ beam). To ensure that the field-ionized fragments are drawn up into the center of the TOF spectrometer, it is desirable to perform the photoexcitation upstream of the spectrometer axis. For this reason, the spectrometer was translatable along the molecular beam axis (under vacuum). The spectrometer geometry is similar to that used for MATI by Jouvet and co-workers [94].

Once the field-ionizing and extraction pulse is applied, the ions are drawn up into the TOF spectrometer, which is illustrated in Fig. 2.2. For good mass-resolution the Wiley-McLaren [95] conditions are used for space focusing. For a given spectrometer geometry, these conditions constrain the ratio of fields in regions P2-P3 and P3-P4 in a manner which helps minimize the spread in TOF's due to the vertical spread in initial ion starting positions. Thus if the extraction pulse strength is changed, it is necessary to 
vary the flight tube voltage to maintain Wiley McLaren conditions. Deflection plates are placed between $\mathrm{P} 4$ and the flight tube to help compensate for molecular beam velocity in the direction orthogonal to the spectrometer.

As discussed in the first chapter, the speed at which an electric-field pulse is applied to a Rydberg atom, will influence its mode of ionization. Similar considerations will apply to the electric-field induced dissociation of high- $v$ ion-pair states. All voltage pulses applied in this work had rise-times of less than 50 ns. However, more detailed characterizations were not made.

\subsubsection{Detection Electronics}

After traveling down the TOF tube, the ions hit the front of a microchannel plate detector [96]. The wiring of this detector is similar to that presented in Fig. 2.14b of Ref. [62] (only all capacitors are $2 \mathrm{nF}$ ). The multiplied electron signal is collected by an anode, and generates a voltage across a $50 \Omega$ resistor, which is then amplified by a fast $\times 10$ preamp. The appropriate ion-signal is gated and integrated in time, relative to the initial laser firing. The gated integrator (Stanford Research Systems, SR250) holds its output voltage until the next laser shot, allowing slow conversion by a 12 bit A/D card in the data collection computer. The scan program records this signal as a function of the VUV energy, which it changes by varying the wavelength of the $\nu_{2}$ laser through a GPIB interface. The data is then written to disk for further analysis. It has proven useful to simultaneously record the VUV intensity, in addition to the ion-signal. Normalization by VUV intensity often significantly improves the $S / N$ of the collected spectra. 


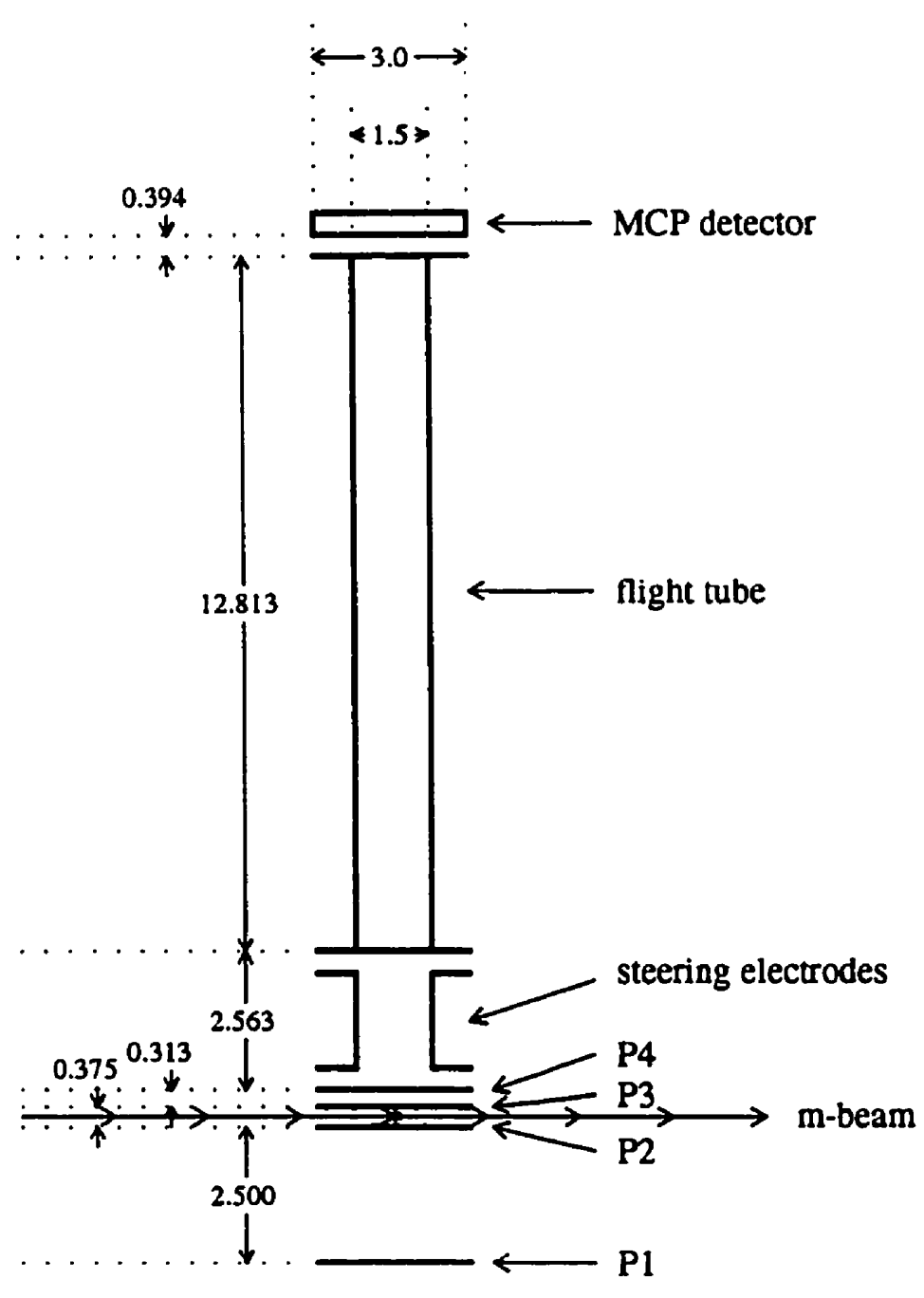

Figure 2.2: Schematic diagram of spectrometer - view from side. All dimensions in inches. The VUV beam travels out of the page, at the point marked *, between P2 and P3. The entire spectrometer is translatable along the molecular beam axis (m-beam) with respect to this point. Plates P2, P3, and P4 are square $\left(3^{\prime \prime} \times 3^{\prime \prime}\right)$ with nickel mesh covered holes, allowing the transmission of ions. The ends of the flight tube $\left(1.5^{\prime \prime} \emptyset\right)$ are also covered with this mesh. The voltage pulse required to generate the discrimination field is applied to P3, for the extraction field it is applied to P2. These are both terminated with $50 \Omega$ resistors to ground. All other electrodes are decoupled through $1 \mathrm{nF}$ ceramic capacitors tied to ground. 


\section{Chapter 3}

\section{Mass Analyzed Threshold}

\section{Ionization Spectroscopy of Argon}

\section{- An Illustrative Example}

\subsection{Introduction}

As mentioned previously, the experimental method for performing TIPPS spectroscopy is virtually identical to mass-analyzed threshold ionization (MATI) spectroscopy $[29,55]$. Thus, to confirm that the newly constructed spectrometer worked, the photoionization of argon was studied at its first $\left({ }^{2} P_{3 / 2}\right)$ and second $\left({ }^{2} P_{1 / 2}\right)$ ionization thresholds. Spectra at the ${ }^{2} P_{1 / 2}$ ionization threshold were studied as a function of the strength of the discrimination field. This enabled extrapolation to determine the field-free ionization threshold. The procedure was then checked against more precise determinations, providing a test of both the VUV energy calibration and extrapolation procedure. This test is the subject of this chapter.

The valence-shell ground-state configuration of $\mathrm{Ar}^{+}$is $3 s^{2} 3 p^{5}$. This configuration has 
a single term ${ }^{2} P$, which has two levels or spin-orbit components: ${ }^{2} P_{3 / 2}$ (lower) and ${ }^{2} P_{1 / 2}$ (upper), separated by $1431.58 \mathrm{~cm}^{-1}$ [97]. The first ionization threshold of Ar lies at $127109.80 \mathrm{~cm}^{-1}$ [98], and thus the second lies at $127109.80+1431.58 \mathrm{~cm}^{-1}=128541.38$ $\mathrm{cm}^{-1}$.

If a photo-yield spectrum of Ar is taken between the first and second ionization thresholds, a series of sharp resonances are observed. These correspond to excitation of Rydberg states with excited ionic-cores $\left({ }^{2} P_{1 / 2}\right)$, which then autoionize. Under certain conditions, if close enough to the second ionization threshold, these Rydberg states may be sufficiently long-lived to allow waiting periods on the order of several $\mu \mathrm{s}$, allowing discrimination against the prompt electrons from the lower (first) ionization threshold. Typically, autoionization lifetimes vary as $n^{3}$, increasing as one approaches the ionization threshold. However, in Ar the $n^{3}$ scaling is not sufficient to explain the experimentally observed long lifetimes [99]. This is discussed more extensively in Appendix B, where the signal strengths of PFI-ZEKE spectra at the first and second ionization thresholds of $\mathrm{Ar}$ are compared to the relative photoionization cross-sections. However, the important point here is that it is possible to collect PFI-ZEKE spectra at the second ionization threshold of Ar, and thus it should also be possible to collect MATI spectra.

Recall that both the TIPPS and MATI technique rely on discrimination against the prompt ions formed during photoexcitation - followed by the selective detection of the ions produced by electric-field induced ionization (MATI) or electric-field induced dissociation (TIPPS). The ratio between the photoionization cross-sections for the two channels at the second ionization threshold of argon is $\sigma\left({ }^{2} P_{3 / 2}\right) / \sigma\left({ }^{2} P_{1 / 2}\right)=1.93$ [100] (see Appendix $B)$. So when taking MATI spectra of argon at the second ionization threshold, there will be at least 1.93 times more ions to be discriminated against than ions formed by fieldionization. ${ }^{1}$ Thus MATI spectroscopy at the second ionization threshold of Ar provides a test of the discrimination properties of the spectrometer with a reasonably high mass

\footnotetext{
${ }^{1}$ There may be more, since some of the Rydberg states approaching the second threshold may autoionize, producing prompt-ions thereby reducing the number of states for field-ionization.
} 
(mass 40).

\subsection{Experimental}

To excite Ar from its ground state to the second ionization threshold in a single photonexcitation, requires $15.9 \mathrm{eV}$ photons. These were generated by four-wave sum-frequency mixing in $\mathrm{Kr}$, as discussed in Section 2.2. The four-wave mixing process was resonantly enhanced by fixing the frequency-doubled output $\nu_{1}$ of a dye laser in the vicinity of a two-photon resonance in $\mathrm{Kr}\left(2 \nu_{1} \approx 94093.7 \mathrm{~cm}^{-1}\right.$ [101]). The dye laser operated using the Stilbene 420 dye (Exciton), and was frequency doubled in a BBO crystal. The tunable frequency $\nu_{2}$ was generated by frequency doubling the output of a Rhoadamine $6 \mathrm{G}$ dye laser in KDP. The two beams were combined using a dichroic coated to reflect $212 \mathrm{~nm}$ at $45^{\circ}$ (Acton Research). The beams were focused with a $20 \mathrm{~cm}$ lens into a free pulsed jet of $\mathrm{Kr}$, and the desired harmonic $2 \nu_{1}+\nu_{2}$ was refocused by the monochromator into the main chamber. Before focusing, the beam waists were on the order of $1-2 \mathrm{~mm}$, and the $\nu_{1}$ energy was $0.5-1 \mathrm{~mJ} / \mathrm{pulse}$, and the $\nu_{2}$ energy was $1.5-2.5 \mathrm{~mJ} / \mathrm{pulse}$.

The Ar backing pressure behind the pulsed valve was 26 psi, and the number density in the interaction region was estimated to be $3 \times 10^{13} \mathrm{~cm}^{-3}$ [102]. During the experiments, the average experimental chamber pressure was typically $10^{-5}$ torr.

The criteria for discrimination against prompt ions has been discussed in Chapter 2. Substituting $m=40$ amu into Eqn. 2.7, gives

$$
F_{d} t_{d}^{2}>41.5 \frac{\mathrm{V}}{\mathrm{cm}}(\mu \mathrm{s})^{2}
$$

where $F_{d}$ is the discrimination field and $t_{d}$ is the discrimination time - the time between the light pulse and extraction field. A discrimination time of $8.4 \mu$ s was used, and thus the discrimination field should be $F_{d} \geq 0.6 \mathrm{~V} / \mathrm{cm}$, for efficient discrimination. In practice it was possible to collect MATI spectra at discrimination fields as low as $2 \mathrm{~V} / \mathrm{cm}$ without significant background. To achieve these rather long discrimination times (the longest for 
any MATI or TIPPS experiment in this thesis), the spectrometer was translated along the molecular beam axis by about $1.4 \mathrm{~cm}$ to ensure that the $\mathrm{Ar}^{+}$ions created at the time of the extraction pulse travelled up the center of the spectrometer (this was empirically adjusted to give the best signal).

After the discrimination time period, an extraction pulse of $60 \mathrm{~V} / \mathrm{cm}$ was applied to the region. The voltage of the TOF tube was varied to maximize the peak $\mathrm{Ar}^{+}$signal seen in the TOF spectrum (ie. the time-resolution of the TOF was optimized). With an optimal TOF tube voltage of $300 \mathrm{~V}$, the $\mathrm{Ar}^{+}$signal was observed approximately 11.1 $\mu s$ after the extraction pulse was applied. After amplification by the microchannel plate detector, the signal was recorded using a boxcar integrator with 30 ns long gate - which was sufficient to record the entire ion signal.

\subsection{MATI Determination of the ${ }^{2} P_{1 / 2}$ Ionization Threshold of $\mathrm{Ar}$}

Figure 3.1 shows a MATI spectrum of Ar at the ${ }^{2} P_{1 / 2}$ ionization threshold taken with a particular discrimination field $(2 \mathrm{~V} / \mathrm{cm})$. The signal corresponds to the detection of $\mathrm{Ar}^{+}$ions originating from the electric field ionization of $\mathrm{Ar}$ Rydberg states with ${ }^{2} P_{1 / 2}$ ion-cores.

When the magnitude of the discrimination field-pulse is increased (Fig. 3.2), the "blueedge" of the MATI peak shifts to lower energies, as the larger discrimination field ionizes lower- $n$ Rydberg states. Rydberg states which have been depleted by the discrimination pulse cannot be detected by the field ionization and extraction pulse. This systematic shifting of the blue-edge with discrimination field will be used to determine the field-free ionization threshold. First, several of the factors affecting the line-shape of the spectra will be reviewed.

The MATI signal strength observed at a particular photon-energy is determined by 


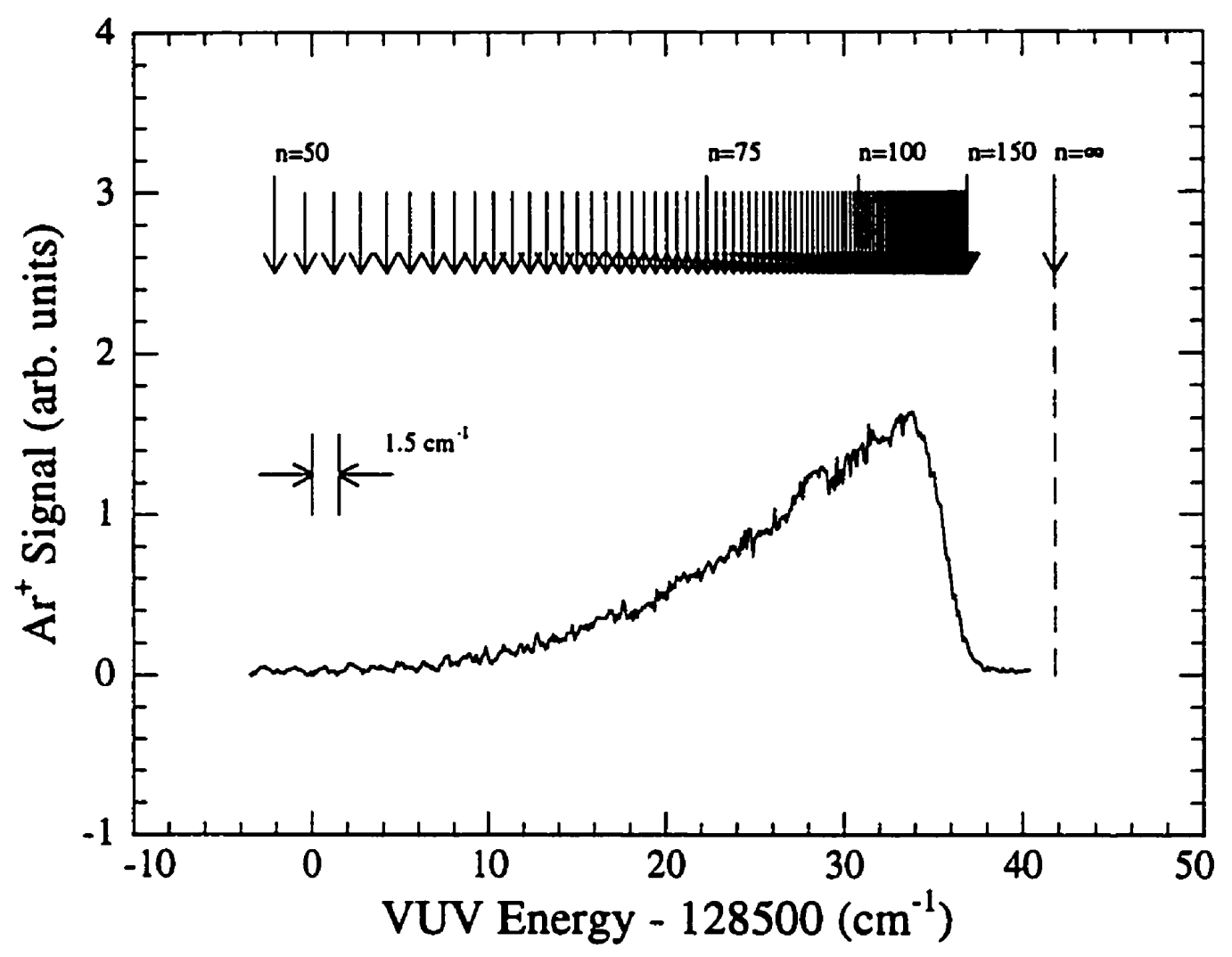

Figure 3.1: MATI spectrum of $\mathrm{Ar}$ at the ${ }^{2} P_{1 / 2}$ ionization threshold. The discrimination pulse was $2 \mathrm{~V} / \mathrm{cm}$ and the extraction pulse was $60 \mathrm{~V} / \mathrm{cm}$. The extrapolated field-free ionization threshold has been marked (see later in this Chapter), as have the line positions of a Rydberg series approaching this threshold (computed using the Rydberg formula with a quantum defect of zero). As the VUV linewidth exceeds the Rydberg state spacing, individual Rydberg states are not spectrally resolved. 


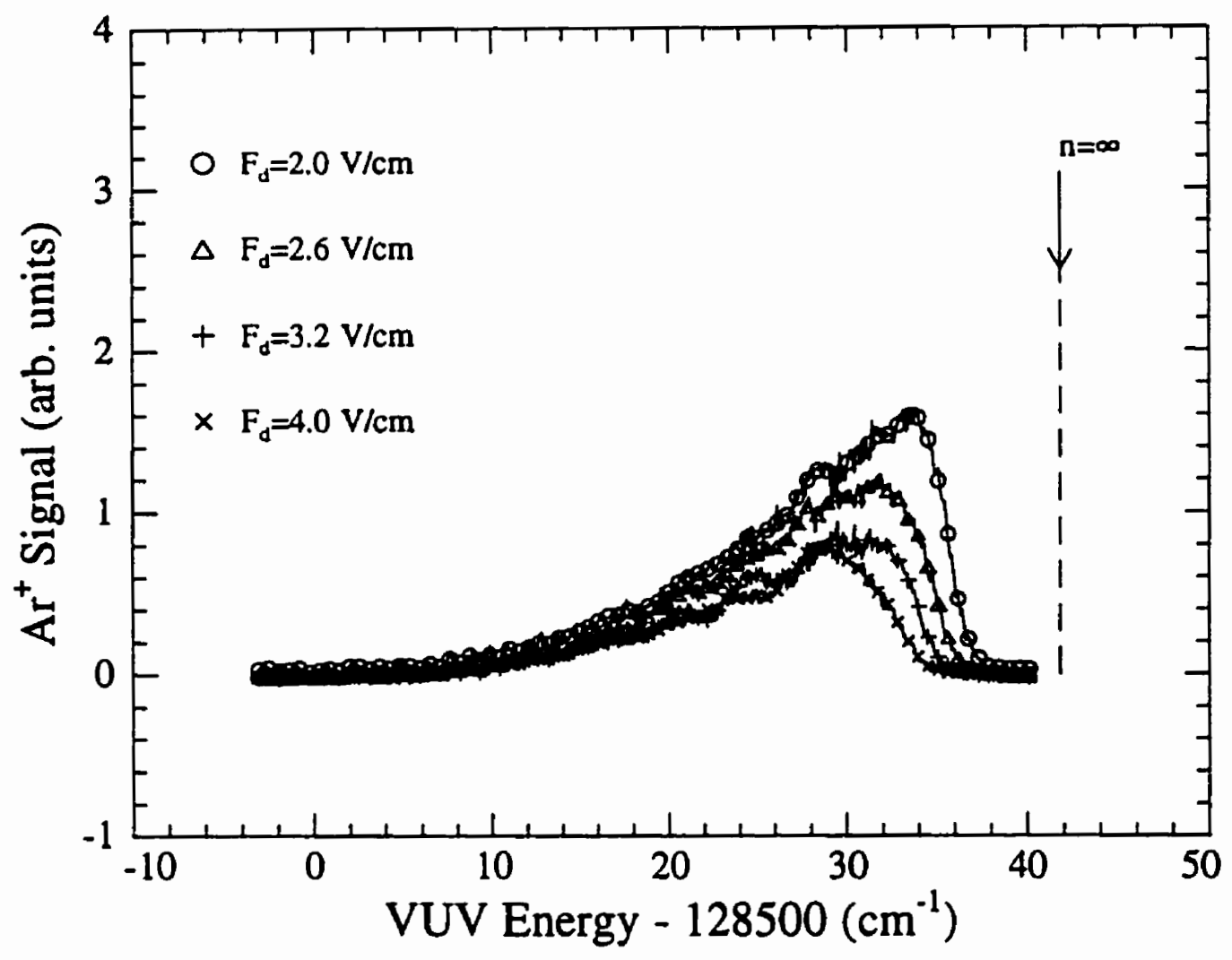

Figure 3.2: MATI spectra of Ar at the ${ }^{2} P_{1 / 2}$ ionization threshold taken with different discrimination fields $\left(F_{d}\right)$. The extraction field strength was $60 \mathrm{~V} / \mathrm{cm}$. These spectra were tested to make sure that their blue edges did not shift as ion-density was reduced. As discussed in the text, intermolecular effects at higher ion-densities deplete the high-n signal. 
several factors:

1. Initial excitation probability. This may vary significantly with energy. In Appendix $B$ the PFI-ZEKE signal strengths observed at the ${ }^{2} P_{1 / 2}$ and ${ }^{2} P_{3 / 2}$ ionization thresholds of Ar are compared to the corresponding partial photoionization cross-sections. In particular, the ${ }^{2} P_{3 / 2}$ ionization cross-section varies strongly in the vicinity of the ${ }^{2} P_{3 / 2}$ ionization threshold (see Fig. B.1).

2. Loss due to autoionization. Rydberg states with excited ionic cores may decay in the waiting period due to autoionization. The Rydberg electron "picks" up ionic core energy and is ejected, and thus does not remain for field-ionization.

3. Loss due to collisions in the waiting period. The very high- $n$ Rydberg states of a MATI or PFI-ZEKE spectrum can be depleted in the waiting period. This is illustrated in Fig. B.3 of Appendix B. This effect is observed to be dependent on ion-density, suggesting that collisions with ions destroy the Rydberg states, and thus they do not remain for field-ionization by the extraction field. To avoid these effects, ion-density can be reduced, unfortunately at the expense of reduced signal. For this reason, it would be nice to control the spot size of the VUV in the experimental region.

4. Survival through the discrimination field. The purpose of the discrimination field is to push the prompt ions out of the detection region. However, use of this weak field also causes ionization of the highest- $n$ Rydberg states. Since the depletion due to this pulse is $n$-dependent (see the next item), this effects the lineshape.

5. Probability of ionization by the extraction field. A pulsed electric field may only be partially efficient in field ionizing all of the optically prepared states at a specific energy. This was discussed in Chapter 1 , and was nicely illustrated by Merkt et al. [103], who showed that for a certain range of $n$ below threshold it is possible to apply multiple successive electric-field pulses, of the same magnitude and duration, 
and obtain multiple field ionization signals. In other words, the first and subsequent pulses are not $100 \%$ efficient in field ionization.

In Chapter 1 it was discussed how the critical ionization fields $\left(F_{c}\right)$ scale like $F_{c} \propto(E-$ $\left.E_{\text {thres }}\right)^{2}$ for $E<E_{\text {thres, }}$, where $E$ is excitation energy, and $E_{\text {thres }}$ is the field-free ionization threshold. Thus one would expect that the field-ionization efficiency, $P_{\text {field-ionization, }}$ might scale like: ${ }^{2}$

$$
P_{\text {field-ionization }}=\Phi\left(\frac{E-E_{\text {thres }}}{\sqrt{F}}\right),
$$

where $\Phi$ is a universal function (applicable for all $F$ ). The discrimination-field survival probability is then:

$$
\begin{aligned}
P_{\text {survival }} & =1-P_{\text {field-ionization }} \\
& =1-\Phi\left(\frac{E-E_{\text {thres }}}{\sqrt{F_{d}}}\right) .
\end{aligned}
$$

Assuming that the variation in this survival probability is the only factor determining the shape of the blue-edge of the MATI lines, an extrapolation scheme for determining $E_{t h r e s}$ may be devised. By empirically judging (for a specific discrimination field strength) the energy at which we are $50 \%$ "up" the blue edge of the MATI peak, $E_{1 / 2}$, and tabulating this position for a series of different discrimination fields, it should be possible to extrapolate to zero-field strength. At zero field strength, $E_{1 / 2}$ should coincide with Ethres.

How should the extrapolation to zero field strength be performed? Since the nature of the function $\Phi$ does not depend on $F_{d}$, the argument which produces $\Phi=1 / 2$ is constant for all $F_{d}$. Referring to this value as $-\alpha$,

$$
-\alpha=\frac{E_{1 / 2}-E_{\text {thres }}}{\sqrt{F_{d}}}
$$

or

$$
E_{1 / 2}=E_{\text {thres }}-\alpha \sqrt{F_{d}}
$$

\footnotetext{
${ }^{2}$ This scaling of field-ionization efficiency has been experimentally observed in the PFI-ZEKE spectra of Merkt et al.; see Fig. 1 of Ref. [103].
} 
Thus a plot of $E_{1 / 2}$ vs. $\sqrt{F_{d}}$ should yield a straight-line with a slope of $-\alpha$, and an intercept of $E_{\text {thres. }}$.

In Fig. 3.3, values of $E_{1 / 2}$, corresponding to the spectra shown in Fig. $3.2,{ }^{3}$ have been plotted as a function of $\sqrt{F_{d}}$. A least-squares straight-line has been fitted to these points, and the intercept, or $E_{\text {thres }}$ has been determined to be $128542.4 \pm 0.5 \mathrm{~cm}^{-1}$. Before comparing this with the literature value, the field-free threshold is determined in another manner.

\subsection{Rydberg State Extrapolation to Determine the ${ }^{2} P_{1 / 2}$ Ionization Threshold of Ar}

If total $\mathrm{Ar}^{+}$ion signal is recorded as a function of energy between the first and second ionization thresholds of $\mathrm{Ar}$, a series of sharp resonances are seen (Fig. 3.4). These correspond to autoionizing Rydberg states with excited ${ }^{2} P_{1 / 2}$ cores, converging on the second threshold $\left(3 p^{5} n s^{\prime}\left(\frac{1}{2}\right)_{1}^{0} \leftarrow 3 p^{61} S_{0}\right)$. By tabulating the energy positions of the observed resonances, it is possible to determine the limit of this series - the ${ }^{2} P_{1 / 2}$ ionization threshold.

To find the limit of the series, $E_{\text {thres, }}$, the procedure of Yoshino and Freeman [104] was followed. Assume that the energy positions of the Rydberg series ( $E_{\text {obs }}$ ) are given by:

$$
E_{o b s}=E_{t h r e s}-\frac{R}{(n+a)^{2}},
$$

where $n$ is an integer, $a$ is a constant, and $R$ is the Rydberg constant. A plot of $E_{o b s}+\frac{R}{(n+a)^{2}}$ should then be constant (and equal to $E_{\text {thres }}$ ) as a function of $(n+a)^{-2}$. The problem is that $a$ is not known $a$ priori. However, $a$ can be adjusted until it is observed that $E_{o b s}+\frac{R}{(n+a)^{2}}$ is constant as a function of $(n+a)^{-2}$, and consequently $E_{\text {thres }}$ determined. Figure 3.5 demonstrates this, where $E_{\text {obs }}+\frac{R}{(n+a)^{2}}$ is plotted for several trial values of $a$ (Table 3.1 lists the energy positions used in the extrapolation).

\footnotetext{
${ }^{3}$ These points were checked, to make sure that they did not shift as ion-density was varied.
} 


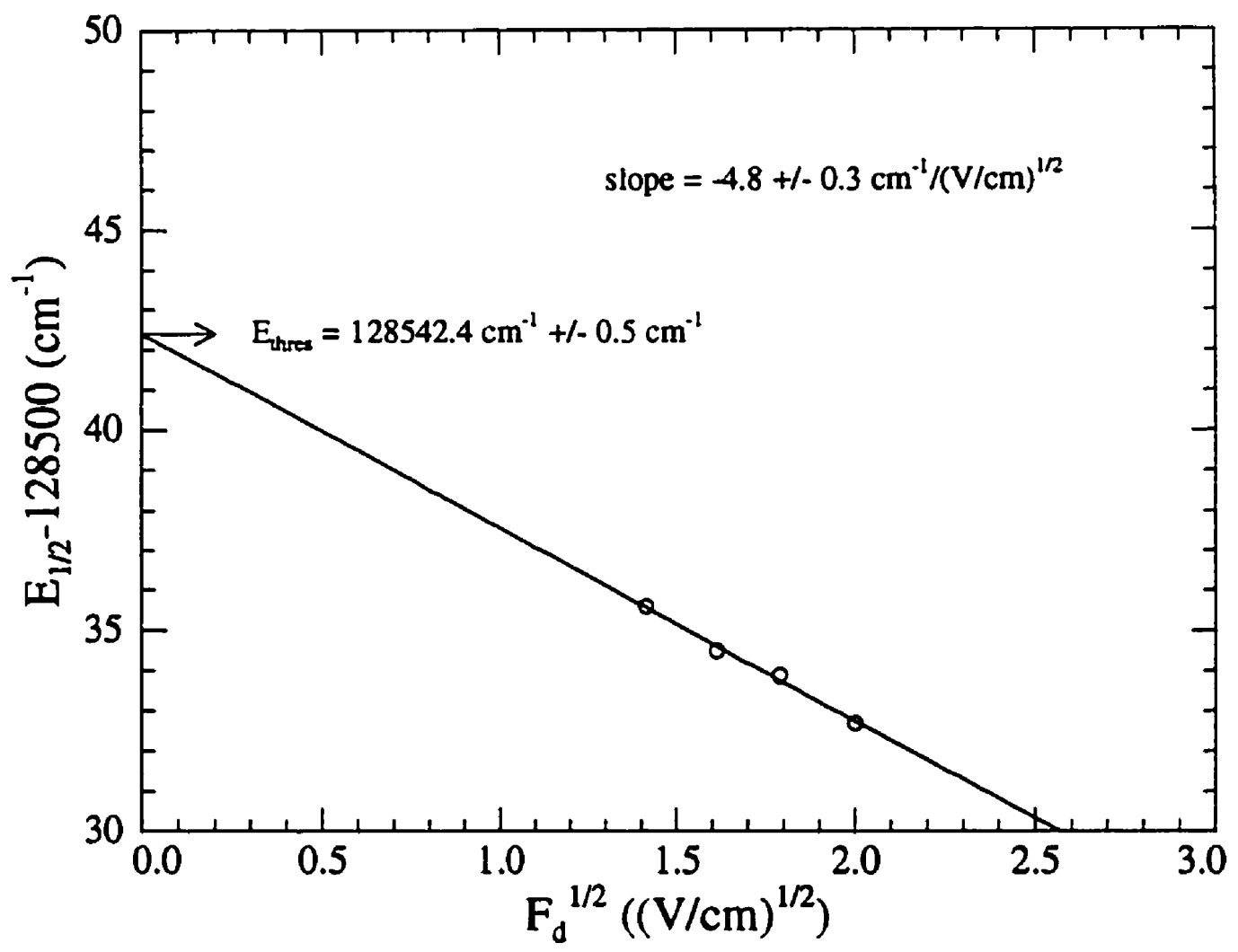

Figure 3.3: Extrapolation to determine the field-free ${ }^{2} P_{1 / 2}$ ionization threshold of Ar. The $E_{1 / 2}$ positions of a series of spectra, taken at different discrimination fields, are plotted as a function of the square root of the discrimination field. A least-squares straight line fit has been superimposed. 


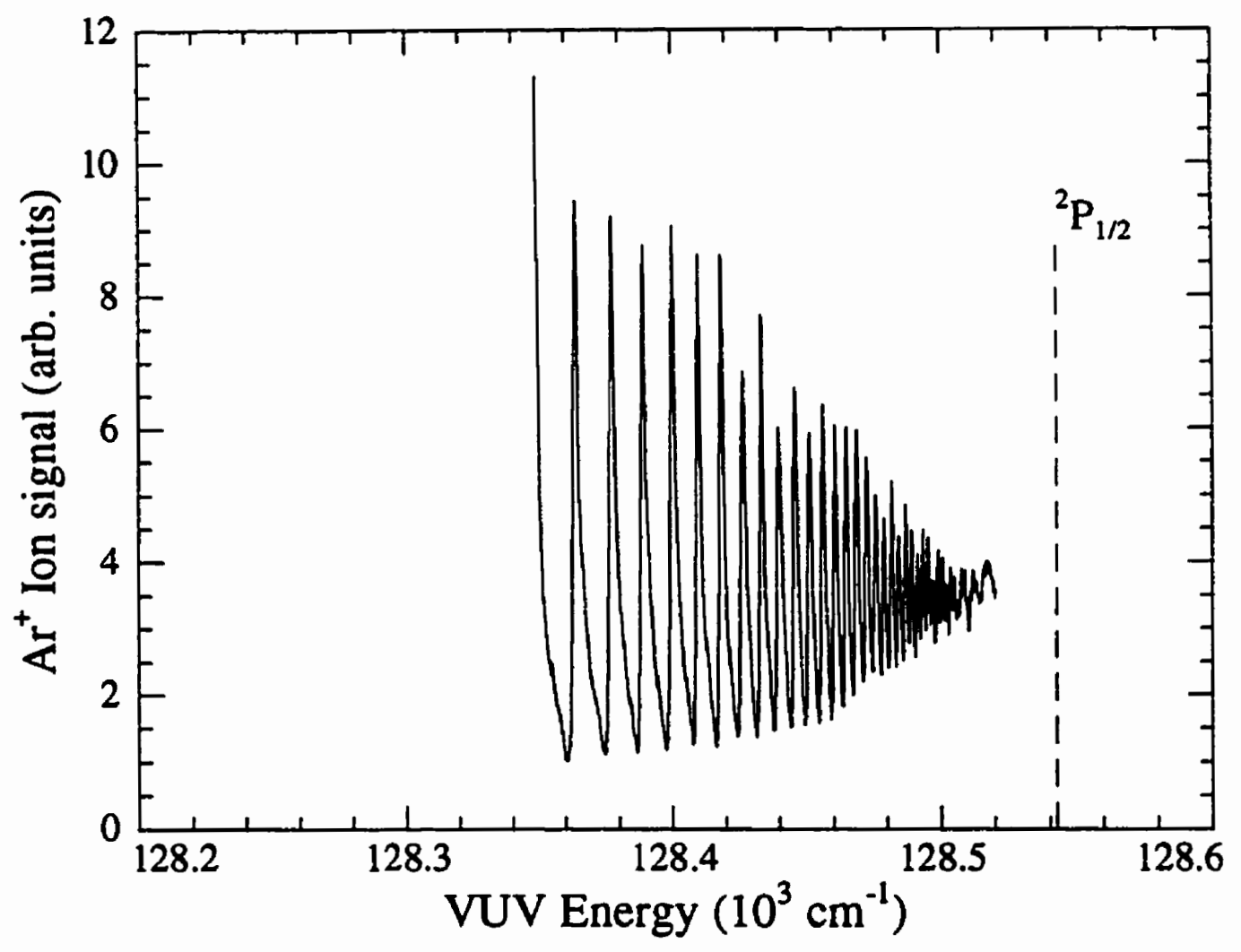

Figure 3.4: $\mathrm{Ar}^{+}$total ion yield between the first and second ionization thresholds. Total ions were collected by pulsing on an extraction field $(60 \mathrm{~V} / \mathrm{cm}$ ) after photoexcitation (no discrimination field was used). The energy position of the extrapolated ionization limit $\left({ }^{2} P_{1 / 2}\right)$ is indicated. The high lying states are unresolved because of the finite resolution of the excitation source. 


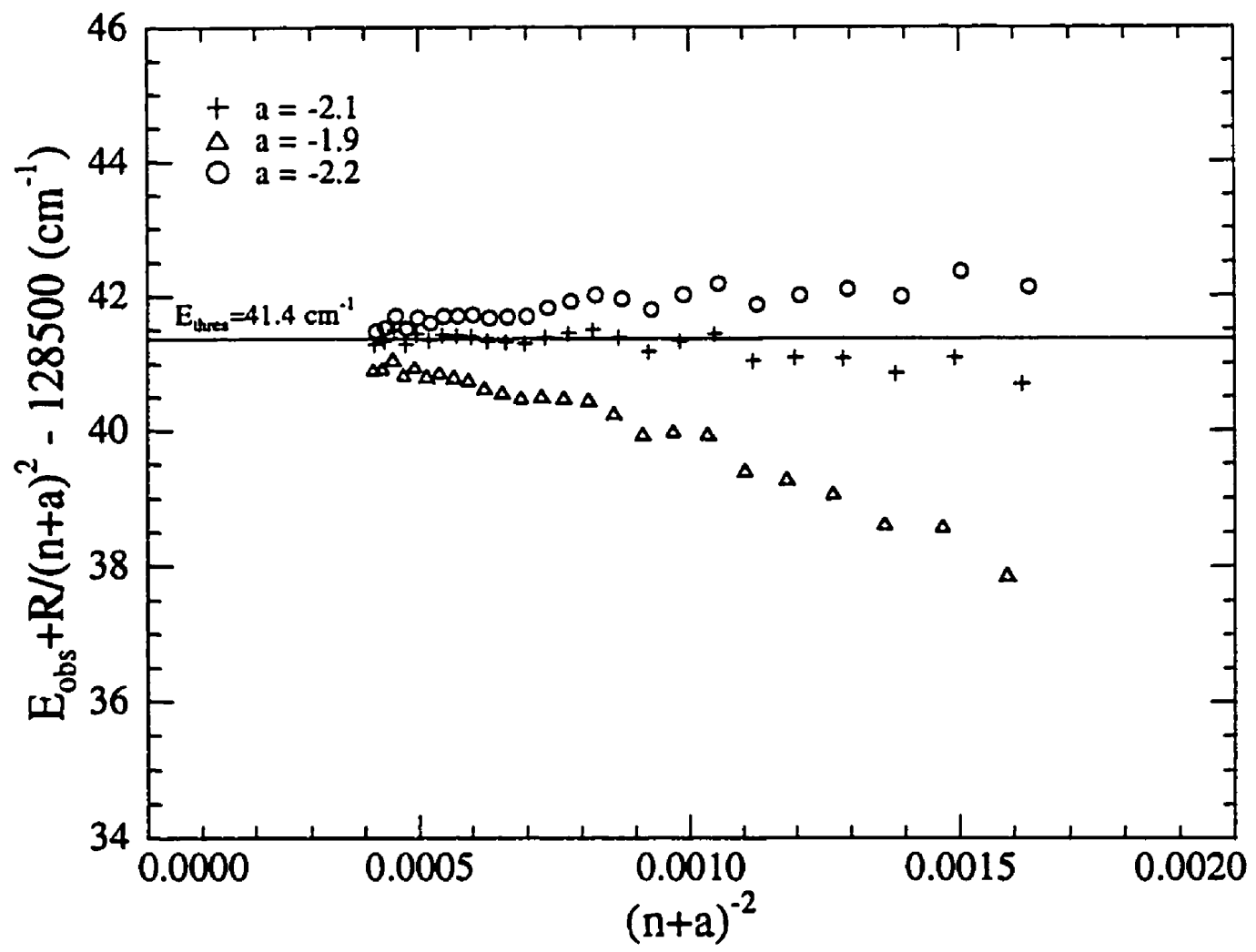

Figure 3.5: Rydberg-state extrapolation to determine the ${ }^{2} P_{1 / 2}$ ionization threshold of Ar. Three trial values of $a$ are shown. The best value of $a$ appears to be $a=-2.1$, which leads to an extrapolated threshold of $128541.4 \mathrm{~cm}^{-1}$. This plot is analogous to Fig. 3 of Ref. [104]. 


\begin{tabular}{|c|c|c|c|}
\hline \hline$n$ & $\begin{array}{c}E_{\text {obs }} \text { (this work) } \\
\left(\mathrm{cm}^{-1}\right)\end{array}$ & $\begin{array}{c}E_{\text {obs }} \text { (Ref.'s [105] and [98]) } \\
\left(\mathrm{cm}^{-1}\right)\end{array}$ & $\begin{array}{c}\text { difference } \\
\left(\mathrm{cm}^{-2}\right)\end{array}$ \\
\hline 27 & 128363.7 & 128364.1 & -0.4 \\
28 & 128377.5 & 128377.6 & -0.1 \\
29 & 128389.2 & 128389.6 & -0.4 \\
30 & 128400.1 & 128400.4 & -0.3 \\
31 & 128409.7 & 128410.0 & -0.3 \\
32 & 128418.3 & 128418.7 & -0.4 \\
33 & 128426.5 & 128426.5 & 0.0 \\
34 & 128433.5 & 128433.7 & -0.2 \\
35 & 128439.8 & 128440.3 & -0.5 \\
36 & 128445.9 & 128446.1 & -0.2 \\
37 & 128451.4 & 128451.5 & -0.1 \\
38 & 128456.3 & 128456.5 & -0.2 \\
39 & 128460.8 & 128461.0 & -0.2 \\
40 & 128464.9 & 128465.4 & -0.5 \\
41 & 128468.8 & 128469.3 & -0.5 \\
42 & 128472.4 & 128472.7 & -0.3 \\
43 & 128475.8 & 128476.2 & -0.4 \\
44 & 128478.9 & 128479.4 & -0.5 \\
45 & 128481.8 & 128482.0 & -0.2 \\
46 & 128484.4 & 128484.8 & -0.4 \\
47 & 128487.0 & 128487.5 & -0.5 \\
48 & 128489.2 & 128489.6 & -0.4 \\
49 & 128491.6 & 128491.6 & 0.0 \\
50 & 128493.5 & 128493.9 & -0.4 \\
51 & 128495.4 & 128495.5 & -0.1 \\
\hline \hline
\end{tabular}

Table 3.1: Line positions of the $3 p^{5} n s^{\prime}\left(\frac{1}{2}\right)_{1}^{0} \leftarrow 3 p^{6}{ }^{1} S_{0}$ Rydberg series of argon. The observed energy positions used for the extrapolation in Fig. 3.5 are tabulated and compared with those of Yoshino [105] (Table IV) and Minnhagen [98] (Table III). The present determinations seem to be systematically lower by an average of $0.3 \mathrm{~cm}^{-1}$. 
The best value of $a=-2.1$ yields an extrapolation limit of $E_{\text {thres }}=128541.4 \mathrm{~cm}^{-1}$. A formal error estimate is difficult to make, but judging from the limits of the two other trial extrapolations shown (which are clearly not correct), the extrapolation error should be less than $0.5 \mathrm{~cm}^{-1}$.

It is important to point out that this extrapolation procedure only works in cases where the Rydberg series is unperturbed i.e. Eqn. 3.6 holds. The classical example of a perturbed Rydberg series is below the the first ionization potential of $\mathrm{H}_{2}$, where Rydberg states approaching the $N^{+}=0$ and $N^{+}=2$ ionization limits interact strongly, requiring some form of deperturbation analysis to determine the ionization threshold [106].

\subsection{Discussion}

In summary, the ${ }^{2} P_{1 / 2}$ ionization threshold was determined to be $128542.4 \pm 0.6 \mathrm{~cm}^{-1}$ using the MATI technique, and $128541.4 \pm 0.6 \mathrm{~cm}^{-1}$ using Rydberg-state extrapolation. How do these compare with the best literature value? Since the ${ }^{2} P_{1 / 2}-{ }^{2} P_{3 / 2}$ energy level difference in the $\mathrm{Ar}^{+}$ion is accurately known [97], the ${ }^{2} P_{1 / 2}$ ionization threshold may be determined by summing this with the first ionization potential of Ar. The most recent determination of the first ionization potential of Ar appears to be that of Minnhagen [98]. ${ }^{4}$ Combining this with the known ${ }^{2} P_{1 / 2}-{ }^{2} P_{3 / 2}$ difference, the ${ }^{2} P_{1 / 2}$ ionization threshold is determined to be $128541.38 \pm 0.1 \mathrm{~cm}^{-1}$. This agrees well with the Rydberg series extrapolation presented in Section 3.4, which gave $128541.4 \pm 0.6 \mathrm{~cm}^{-1}$. The value obtained by MATI extrapolation is somewhat higher (by $1 \mathrm{~cm}^{-1}$ ), but certainly not unreasonable considering the error estimates. In retrospect, it would have been desirable to take spectra with higher discrimination fields, to confirm that the $\sqrt{F_{d}}$ scaling was valid over a larger range (as was done with $\mathrm{HCl}$ ). However, based on this limited amount of data, the extrapolation procedure seems reasonable, and in subsequent chapters, an

\footnotetext{
${ }^{4}$ A compilation of atomic ionization potentials, with references, is available through the NIST webpage: http://physics.nist.gov/PhysRefData/contents.htal.
} 
analogous procedure will be applied to TIPPS spectra to determine the field-free threshold for ion-pair formation. 


\section{Chapter 4}

\section{Threshold Ion-Pair Spectroscopy of $\mathrm{HCl}$ : An Improved $D_{0}(\mathrm{HCl})$}

\subsection{Introduction}

Although the dissociation energies of several diatomics are known to "spectroscopic" accuracy $\left( \pm 1 \mathrm{~cm}^{-1}\right)$, many are not. An example is $\mathrm{HCl}$, where the oft-quoted value of $D_{0}(\mathrm{HCl})$ in Huber and Herzberg [107], is based on a thermochemical measurement made by Rossini in 1932 [108]. Thus a study of threshold ion-pair formation in $\mathrm{HCl}$ was undertaken, with the specific goal of determining $D_{0}(\mathrm{HCl})$. Since the TIPPS spectra of $\mathrm{HCl}$ is more easily interpretable than either $\mathrm{O}_{2}$ or $\mathrm{HF}$, it is presented first, in this chapter.

The first observation of photoion-pair formation in $\mathrm{HCl}$ was made by Yencha et al. using synchrotron radiation [35]. They characterized this process by collecting total $\mathrm{Cl}^{-}$ ions as a function of photon-energy from the energetic threshold at $14.4 \mathrm{eV}$ to $16.3 \mathrm{eV}$. The structure in this spectrum was modeled theoretically as an indirect process: Rydberg states are excited, which then predissociate into ion-pairs. This analysis was far from trivial, and only an overview may be given here (a useful discussion of these types of 
calculations is given in Ref. [109]).

\subsection{Mechanism for the Formation of Ion-Pairs}

In this section, the mechanism for production of $\mathrm{HCl}$ ion-pairs due to Yencha et al. [35] is reviewed. There are two major factors to consider when analyzing which Rydberg states may contribute to the photoion-pair yield:

1. Can the state be excited? (Is it bright?) In the present case, it must be possible to excite the state from the $X^{1} \Sigma$ ground-state of $\mathrm{HCl}$.

2. Will the Rydberg state predissociate into ion-pairs? In general, the distinction between ion-pair states and Rydberg states is an artificial one - they are coupled by the full Hamiltonian. However, in attempting to understand ion-pair formation as a two-step process we "decouple" them, then consider the perturbations which connect them. At the very high energies typical of ion-pair formation, there are normally several alternative dissociation channels available (into neutrals), and if above the ionization threshold (as for $\mathrm{HCl}$ ), many ionization channels. The rates of all autoionization and predissociation processes must be considered, since they will compete for the fate of the Rydberg states, and contribute to the total linewidth.

$\mathrm{HCl}$ has a ${ }^{1} \Sigma$ ground-state, so single-photon excitation can only result in ${ }^{1} \Sigma$ or ${ }^{1} \Pi$ Rydberg states (in a first approximation). There are a variety of ways that a Rydberg electron and ionic-core can couple to produce these states. However, at these energies the ion-cores states must be either $X^{2} \Pi$ or $A^{2} \Sigma$ (see Fig. 4.1).

Since the $X^{2} \Pi$ ionization threshold is below the threshold for ion-pair formation, vibrationally excited $X^{2} \Pi$ cores must be considered. However, even at the lowest possible $v$, the Franck-Condon factors from the ground state to these vibrational states are prohibitive. Thus excitation must be to either a ${ }^{1} \Sigma$ or ${ }^{1} \Pi$ state with a $A^{2} \Sigma$ ionic core. The 


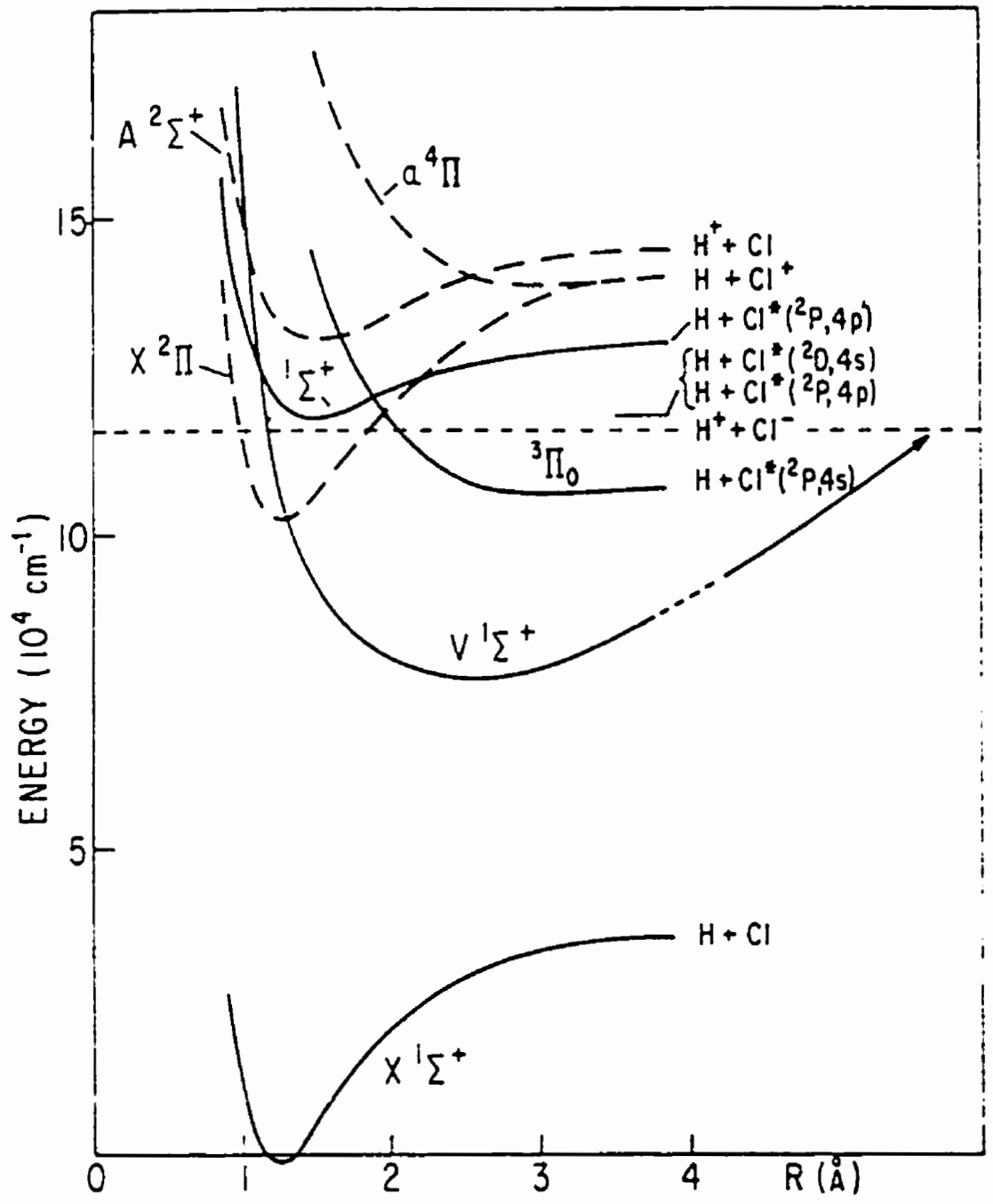

Figure 4.1: Potential energy curves of $\mathrm{HCl}$ and $\mathrm{HCl}^{+}$. From Ref. [35]. The ionic states are represented by dashed lines. 
nature of the Rydberg orbital is now constrained: for the ${ }^{1} \Pi$ states, the orbital must be $\pi$, and for the ${ }^{1} \Sigma$ states, the orbital must be $\sigma$. It is now possible to pick between these two choices, based on ease of predissociation (item 2 above). There is only one state which asymptotically produces the ion-pairs: the $V^{1} \Sigma$ state (the predissociated ion-pairs are in the vibrational continuum of this state). The $V^{1} \Sigma$ and ${ }^{1} \Pi$ states can be coupled through a rotational interaction sometimes called the "gyroscopic" perturbation (see section 2.5.3 of Ref. [110]). But it is argued by Yencha et al. [35] that the matrix elements of this coupling are small compared to the electrostatic coupling of the ${ }^{1} \Sigma$ Rydberg states to the $V^{1} \Sigma$ ion-pair state. Thus, all of the resonances in the ion-pair yield spectrum of $\mathrm{HCl}$ are interpreted as originating from excitation to ${ }^{1} \Sigma$ Rydberg states with $A^{2} \Sigma$ ionic cores, which are then predissociated by electrostatic interaction with the $V^{1} \Sigma$ ion-pair state. The Rydberg electron may still be in a variety of states, designated by: $n l \sigma$. Which of these states dominates the photoion-pair yield spectrum is dictated by both the transition dipole moment and the electrostatic coupling matrix elements. Franck-Condon factors control the allowable vibrational levels of the ionic core. All of these factors are taken into account in the spectral simulation, presented by Yencha et al. [35].

The agreement between the results of these theoretical calculations and the experimental measurements is reasonable (see Fig. 4 of Ref. [35]), suggesting that these are the Rydberg states which are primarily responsible for the photoion-pair yield. However, the authors acknowledge that their experimental spectra have a broad underlying background which is not explained by their calculations - hinting that direct excitation to $V^{1} \Sigma$ ion-pair state might also be possible.

\subsection{Experimental}

The approximately $14.4 \mathrm{eV}$ photons used to study the threshold region were generated by four-wave sum-frequency mixing in $\mathrm{Xe}$, as discussed in Chapter 2. The four-wave mixing process was resonantly enhanced by fixing the frequency-doubled output $\nu_{1}$ of a 
dye laser in the vicinity of a two-photon resonance in $\mathrm{Xe}\left(2 \nu_{1} \approx 80119.5 \mathrm{~cm}^{-1}\right)$. This dye laser operated using the Coumarin 500 dye and was frequency doubled in a BBO crystal. The tunable frequency $\nu_{2}$ was generated by frequency doubling the output of a Fluorescin 548 dye laser in KDP. The frequencies $\nu_{1}$ and $\nu_{2}$ were sufficiently close so as to cause some problems with overlap using a dichroic coated for $45^{\circ}$ reflection of $250 \mathrm{~nm}$ (the $\nu_{2}$ beam was not sufficiently transmitted). The solution was to use a dichroic coated for $212 \mathrm{~nm}$ reflection at $45^{\circ}$, for reflecting $\nu_{1}$ at near normal incidence, while allowing $\nu_{2}$ to be transmitted. Reflection of $\nu_{1}$ was near $100 \%$ efficient, whereas only $50 \%$ of $\nu_{2}$ was transmitted. This was suboptimal, but worked sufficiently well, allowing 1-1.5 mJ/pulse of $\nu_{1}$ and 4-5 mJ/pulse of $\nu_{2}$ to enter the mixing chamber. Both beams were were focused into a free-jet of $\mathrm{Xe}$, and the desired harmonic, $2 \nu_{1}+\nu_{2}$ was collected and refocused into the main chamber by the monochromator.

The $\mathrm{HCl}$ gas was expanded unseeded from a $1 \mathrm{~mm}$ diameter pulsed valve nozzle, with a backing pressure of 20 psi. It travelled $5.2 \mathrm{~cm}$ before interacting with the VUV beam. With the molecular beam on, the average main chamber pressure was $1 \times 10^{-5}$ torr.

Because of their low mass, prompt $\mathrm{H}^{+}$ions are relatively easy to discriminate against. The delay between the light pulse and extraction field for the TIPPS spectra was typically $4.4 \mu \mathrm{s}$. With this timing, discrimination fields as low as $1.5 \mathrm{~V} / \mathrm{cm}$ could be used. To compensate for the movement of the excited states along the beam axis during the discrimination time period, the spectrometer was translated along the molecular beam axis by $0.6 \mathrm{~cm}$.

\subsection{Results and Discussion}

Figure 4.2a) shows the prompt photoion-pair yield spectrum for the process: $\mathrm{HCl}+h \nu \rightarrow$ $\mathrm{H}^{+}+\mathrm{Cl}^{-}$, in the vicinity of the threshold region. This covers a much smaller energy range than that of Yencha and co-workers [35], but at higher resolution $\left(1.5 \mathrm{~cm}^{-1}\right.$ vs. 
$120 \mathrm{~cm}^{-1}$ ). Spectra taken over a larger range would allow a critical test of the calculations presented in their paper.

No attempt was made to ensure $100 \%$ collection efficiency of the prompt $\mathrm{H}^{+}$ions. Thus, it is expected that collection efficiency decreases as the energy is increased above threshold. $\left(\mathrm{H}^{+}\right.$fragments with kinetic energy will leave the region before they can be detected.) This was verified by observing the change in the spectrum as the pulsedfield was delayed further with respect to the photoexcitation. Collecting $\mathrm{Cl}^{-}$fragments would make this task easier. Another artifact is the contribution due to high- $v$ ion-pair states being dissociated by the electric-field, and subsequently detected together with the prompt fragments (these are not technically part of the "free" photoion-pair yield).

However, the spectra shown in Fig. 4.2a) do illustrate two important points associated with the TIPPS experiments. First, it is clear that an identification of the energetic threshold from rotational "steps", 1 such as made in the case of $\mathrm{O}_{2}$ [37] would be impossible. The "steps" are simply not observable (we will see that the situation is even worse for HF). Secondly, the photoion-pair yield signal is not strongly energy dependent, suggesting that the TIPPS signal will be detectable, and won't show sharp energy dependencies. The broad background noted by Yencha et al. [35] is also present at our higher resolution, thus the possibility of direct excitation from the ground electronic state to the $V^{1} \Sigma$ state may be responsible for a portion of the signal.

To collect a threshold ion-pair production (TIPPS) spectrum, a discrimination field of $2 \mathrm{~V} / \mathrm{cm}$ is inserted between the excitation and pulsed extraction fields. This discrimination field is pulsed on $0.3 \mu$ s following photoexcitation, followed by the $60 \mathrm{~V} / \mathrm{cm}$ extraction and field-ionization pulse $4.1 \mu$ s later. Figure $4.2 \mathrm{~b}$ ) shows a TIPPS spectrum obtained by varying the photon excitation energy and recording the gated $\mathrm{H}^{+}$pulsed field dissociation signal. Each of the isolated peaks correspond to a range of unresolved

\footnotetext{
${ }^{1}$ These steps were discussed in Section 1.5.1. They are due to the increase in signal above the threshold for dissociating each initial $\mathrm{J}$ state. They should be at approximately the same locations as the energetic thresholds marked in 4.2b).
} 


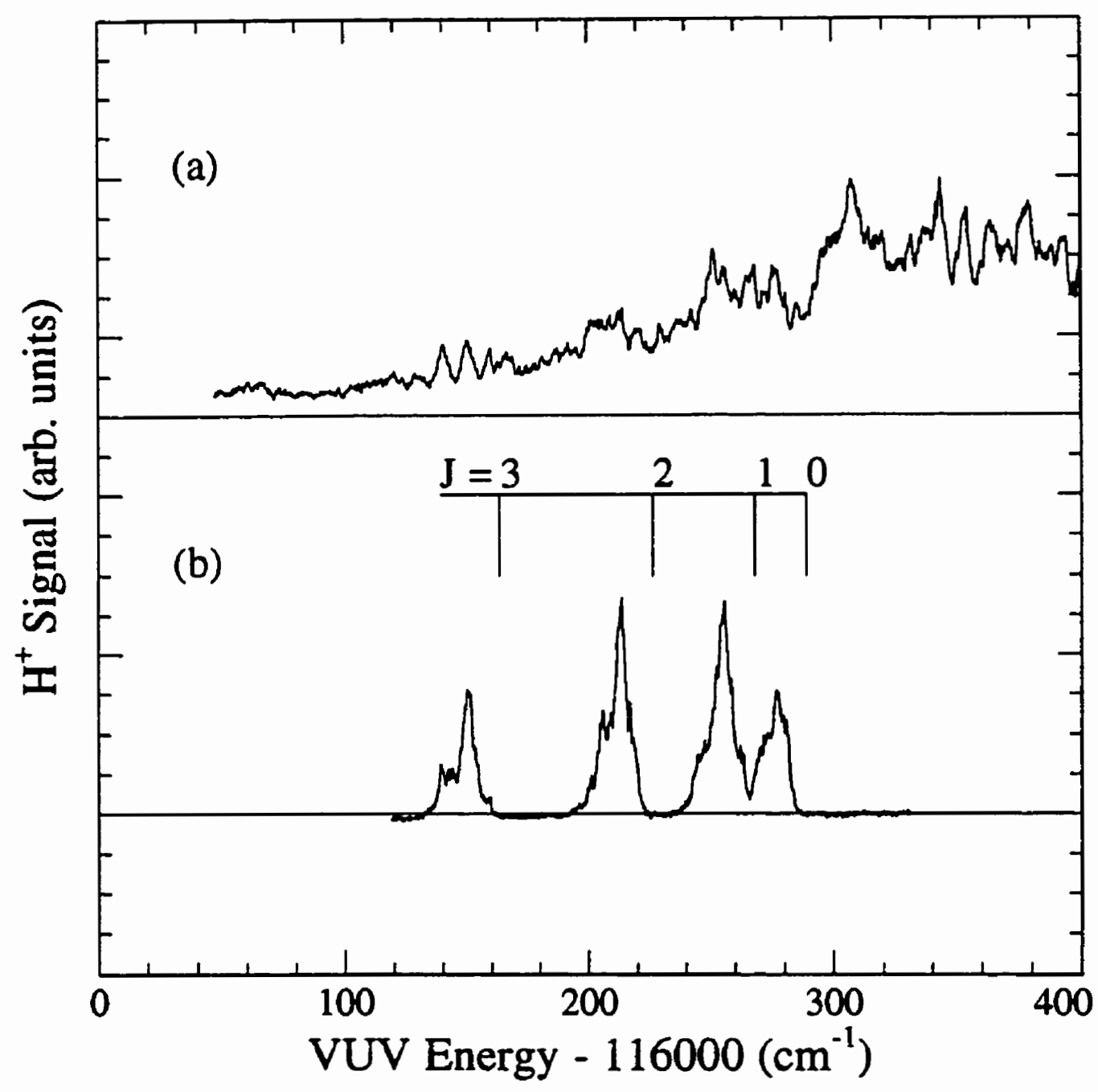

Figure 4.2: (a) Prompt photoion-pair yield from $\mathrm{HCl}$. An extraction field of $60 \mathrm{~V} / \mathrm{cm}$ was pulsed on $0.2 \mu$ s following photoexcitation. No discrimination field was used. No attempt was made to ensure $100 \%$ collection efficiency for fast $\mathrm{H}^{+}$. (b) TIPPS spectrum of $\mathrm{HCl}$. The discrimination field was $2 \mathrm{~V} / \mathrm{cm}$, pulsed on $0.3 \mu$ s following photoexcitation. The extraction field was $60 \mathrm{~V} / \mathrm{cm}$, pulsed on $4.1 \mu \mathrm{s}$ after the discrimination field. The field-free ion-pair formation thresholds for each initial $J$ level have been superimposed (the determination of these is described later in Section 4.5). 
ion-pair vibrational levels converging on the first ion-pair dissociation asymptote, and the individual lines arise from the different initial thermally populated rotational levels of $\mathrm{HCl}$. Since the dissociation asymptote is independent of rotation, the spacing of the peaks is given by the well known rotational constants of $\mathrm{HCl}$ [107]. This spacing has been superimposed on Fig. 4.2b).

In addition to providing discrimination against prompt ions, the first field also has the effect of field-dissociating the very high- $v$ ion-pair states close to the dissociation threshold. This can be confirmed by varying the strength of the discrimination field. Fig. 4.3 shows two TIPPS spectra taken with different discrimination fields. As expected, increasing the discrimination field pushes the blue edge down in energy, since larger fields deplete more of the higher- $v$ ion-pair states. This systematic shifting of the blue-edge with varying discrimination field strength will be used in Section 4.5 to determine the field-free ion-pair dissociation threshold.

What happens when the extraction and field dissociation pulse field strengths are changed? One would expect that larger pulses would shift the red side of the TIPPS peaks to lower energies, broadening the peak. Figure 4.4 shows the effect of varying the strength of the extraction field from $15 \mathrm{~V} / \mathrm{cm}$ to $100 \mathrm{~V} / \mathrm{cm}$ while leaving the discrimination field constant $(4 \mathrm{~V} / \mathrm{cm})$, and keeping the timing of the fields the same. Because the field-dissociation and extraction pulse is the first field in the two-field Wiley-McLaren focusing arrangement, the strength of the second field must be varied, to maintain the space-focusing condition. Generally, as the strengths of these fields are reduced, the iondetection efficiency decreases. However, since this should not depend on photon energy, the $15 \mathrm{~V} / \mathrm{cm}$ and $100 \mathrm{~V} / \mathrm{cm}$ spectra have been normalized in Fig. 4.4 so that their maxima match, allowing easier comparison between their lineshapes. Surprisingly, the two spectra do not differ significantly.

To explain why the $15 \mathrm{~V} / \mathrm{cm}$ and $100 \mathrm{~V} / \mathrm{cm}$ extraction pulse spectra are similar, it is helpful to recall the discussion of the factors effecting MATI spectroscopy given 


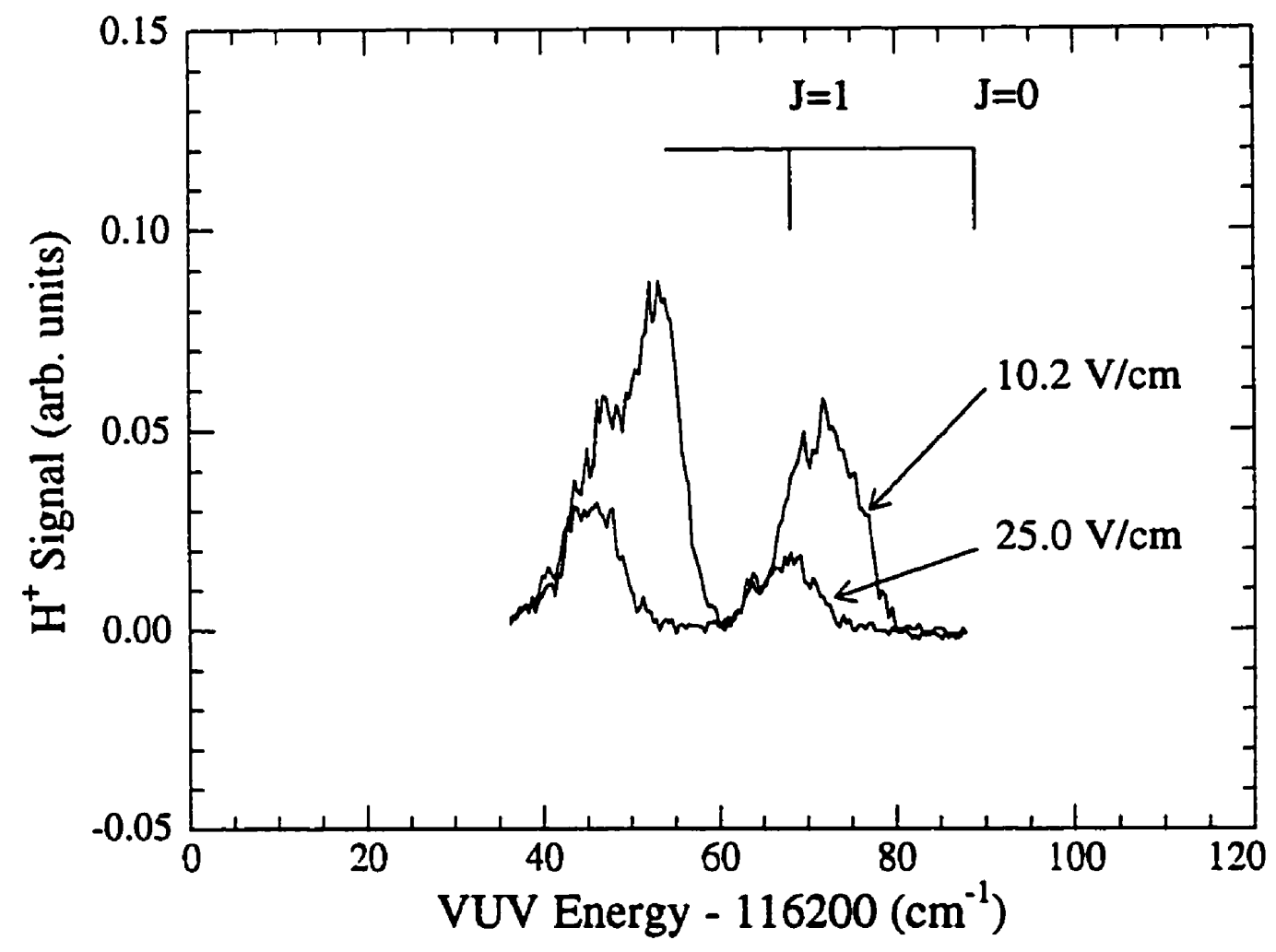

Figure 4.3: TIPPS spectra of $\mathrm{HCl}$ for two different discrimination fields. Conditions are identical to Fig. 4.2, except for the magnitude of the discrimination field. 


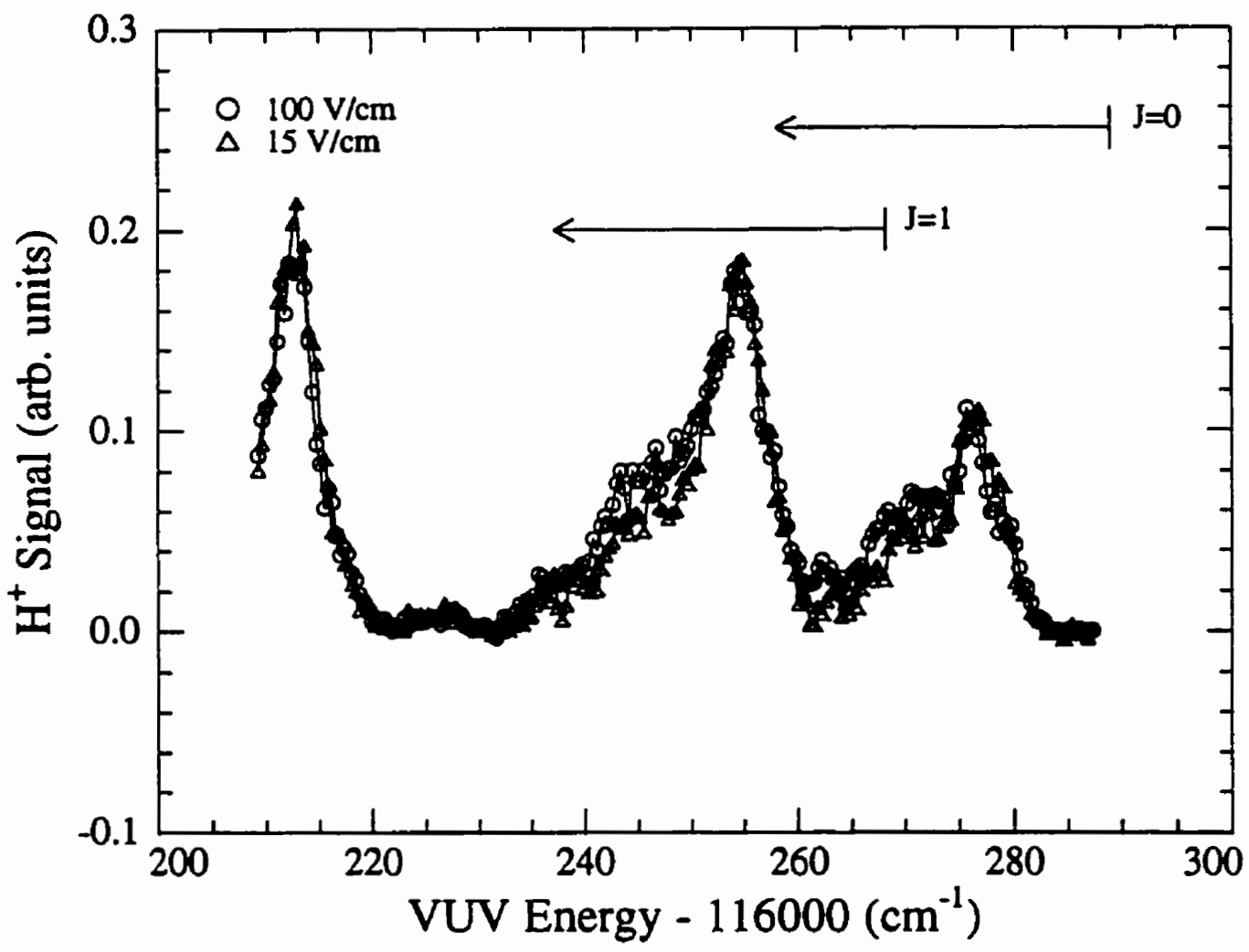

Figure 4.4: $\mathrm{HCl}$ TIPPS spectra taken with a $15 \mathrm{~V} / \mathrm{cm}$ and $100 \mathrm{~V} / \mathrm{cm}$ Extraction Field. The discrimination field pulse was $4 \mathrm{~V} / \mathrm{cm}$, and $1 \mu \mathrm{s}$ long, delayed $0.3 \mu \mathrm{s}$ after photoexcitation. The extraction field was pulsed on $2.4 \mu$ s after the light. The energy range from threshold to $-3.1 \sqrt{100} \mathrm{~cm}^{-1}$ below threshold has been labeled $(\mapsto)$ for both $J=0$ and $J=1$ (see text for discussion). 
in Section 3.3. Analogous considerations apply to TIPPS lineshapes (simply replace "field-ionization" by "field-dissociation"). In particular, the factors influencing TIPPS lineshapes are:

1. Initial excitation probability,

2. Loss due to autoionization or predissociation,

3. Loss due to collisions in the waiting period,

4. Survival through the discrimination field-pulse, and

5. Probability of field-dissociation by the extraction pulse.

Consider 5) first, since this should be affected by changing the extraction pulse strength. Suppose that the field-ionization formulae for diabatic ionization presented in Chapter 1 may be directly applied to the field-dissociation phenomena. Then it would be expected that the field-dissociation efficiency would be $100 \%$ efficient for states with energies between threshold and $3.1 \frac{\mathrm{cm}^{-1}}{(\mathrm{~V} / \mathrm{cm})^{1 / 2}} \sqrt{F}$ below threshold. However this is not the case for the $100 \mathrm{~V} / \mathrm{cm}$ spectra (this limit is marked on Fig. 4.4). Factors 1 or 2 must then be considered as dominating the low-energy side of the lineshapes. (Factor 4 will only affect the high-energy side). Since the ion-pair signal above the threshold is not strongly energy dependent, and more or less identical behavior is observed for both the $J=0$ and $J=1$ lines, it is unlikely that initial excitation probability is reducing the low- $v$ signal. It is much more likely that the lower- $v$ states have decayed somehow, in the waiting period between photoexcitation and the extraction field pulse. Several channels are open for decay: autoionization and dissociation, and in complete analogy with Rydberg states, it would be expected that lower- $v$ ion-pair states would be more susceptible to decay.

If a $v$-dependent decay between photoexcitation and the extraction pulse is the factor determining the shape of the low-energy side of the TIPPS peaks, then it would be expected that the shape of the peaks could be modified by collecting the spectra with 
different delay times between excitation and extraction. A series of spectra taken with different delay times are shown in Fig. 4.5. These spectra do not differ. This is definitely paradoxial, if only the possibility of exponential decay is considered. However it is quite possible that the situation is analogous to that observed in PFI-ZEKE (discussed in Appendix B). The initially excited low- $J$ ion-pair states are highly susceptible to decay (which can happen very quickly), but they may be stabilized by external perturbations. Weak electric fields (possibly due to nearby ions), mix the $J$ quantum number, allowing an initially excited low $J$ state to evolve to a higher $J$ state. The centrifugal barrier would then prevent the positive and negative ions from interacting. Inhomogeneous fields (such as those due to nearby ions), could also be responsible for the mixing of the $M_{J}$ quantum number, favoring higher $J$ states due to their increased $M_{J}$ degeneracy. Thus, initially excited states have two fates: 1 ) stabilization to higher $J$ states by external perturbations (leading to lifetimes much longer than the observation period) or 2) rapid decay (in times much shorter than the observation period). Since the rapid decay process immediately following excitation will be $v$-dependent, this is a plausible explanation for the loss of low- $v$ ion-pair states.

Unfortunately, because it is necessary to discriminate against the prompt ion-pairs (which requires a certain finite time), the shape of spectra at very short times following photoexcitation could not be studied, to test this hypothesis. Further studies are required. Especially important would be confirmation that low- $v$ ion-pair states will decay rapidly in the absence of external perturbations. This type of information was critical to an understanding of the Rydberg state case $[19,99]$, but will be much more difficult to obtain for the high-v ion-pair states.

However, there is one simple experiment that provides some supporting evidence for the stabilization hypothesis. Figure 4.6 shows two TIPPS spectra taken with VUV fluences that differ by a factor of 6 . Varying the VUV fluence, changes the ion-density and 


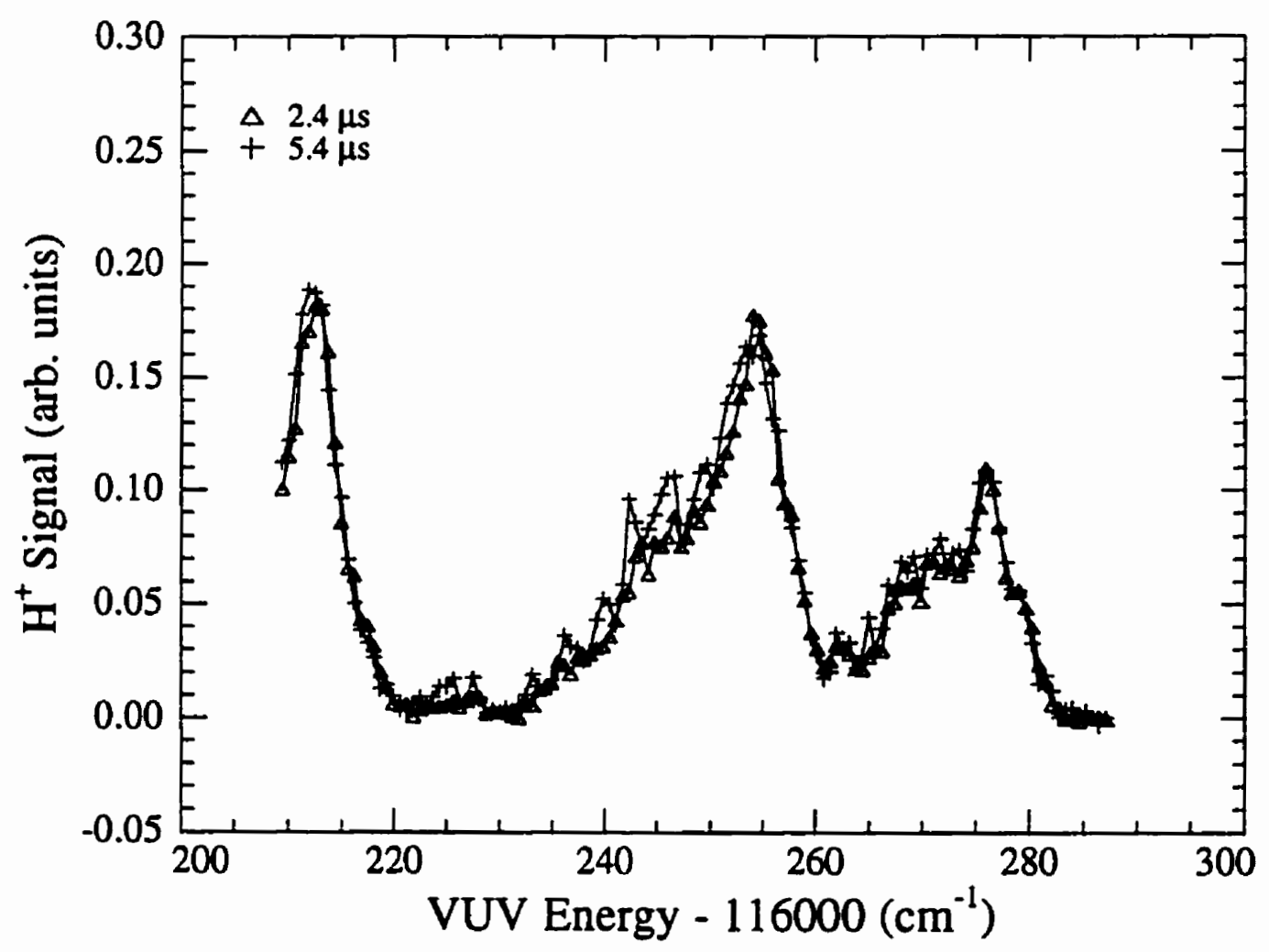

Figure 4.5: $\mathrm{HCl}$ TIPPS spectra for different pulsed field extraction delays. The time between the photoexcitation and extraction pulse is $2.4 \mu \mathrm{s}$, for one spectrum and $5.4 \mu \mathrm{s}$ for the other. Extraction field strength, was $100 \mathrm{~V} / \mathrm{cm}$, and the discrimination field pulse was $4 \mathrm{~V} / \mathrm{cm}$, and $1 \mu \mathrm{s}$ long, pulsed on $0.3 \mu \mathrm{s}$ after photoexcitation. The spectra have been rescaled so that their peaks match. 
thus the external perturbations - which may be responsible for stabilization. ${ }^{2}$ One might then expect that the stabilized "yield" would improve, since stabilization would be more competitive with the autoionization or predissociation losses. This is observed in Fig. 4.6. The low- $v$ signal noticeably improves at higher ion-densities. This is surprisingly similar to what is observed in the PFI-ZEKE spectra of Ar (see Appendix B).

\subsection{Determination of $D_{0}(\mathrm{HCl})$}

\subsubsection{Extrapolation to Determine the Field-Free Ion-Pair Dissociation Threshold}

As mentioned in the introduction to this Chapter, the primary purpose in studying the TIPPS spectra of $\mathrm{HCl}$ was to determine $D_{0}(\mathrm{HCl})$, through the relationship:

$$
D_{0}(\mathrm{HCl})=E_{\mathrm{H}^{+}, \mathrm{Cl}^{-}}-I P(\mathrm{H})+E A(\mathrm{Cl})
$$

where $E_{\mathrm{H}+, \mathrm{Cl}}$ is the field-free ion-pair dissociation threshold, $I P(\mathrm{H})$ is the ionization potential of the hydrogen atom and $E A(\mathrm{Cl})$ is the electron affinity of the chlorine atom. The value of $E_{\mathrm{H}^{+}, \mathrm{Cl}^{-}}$can be obtained by collecting TIPPS spectra with different discrimination fields, in a virtually identical manner to the Ar ionization threshold determination presented in Chapter 3. Since both $I P(\mathrm{H})$ and $E A(\mathrm{Cl})$ are known to better than $1 \mathrm{~cm}^{-1}$, $D_{0}(\mathrm{HCl})$ may be accurately determined.

TIPPS spectra of $\mathrm{HCl}$ were taken at 10.2, 14.4, 19.4 and $25 \mathrm{~V} / \mathrm{cm}$ discrimination field strengths $\left(F_{d}\right)$. For each discrimination field, the value of $E_{1 / 2}$ has been determined (the energy position half-way up the leading blue edge of the line). In analcgy with the MATI spectra discussed in Chapter 3 , it is expected that these would shift with varying $F_{d}$, in the following manner:

$$
E_{1 / 2}=E_{\mathrm{H}^{+}, \mathrm{Cl}^{-}}-k \sqrt{F_{\mathrm{d}}}
$$

\footnotetext{
${ }^{2}$ The major contribution to the ion-density is from photoionization of $\mathrm{HCl}$ producing $\mathrm{HCl}^{+}$.
} 


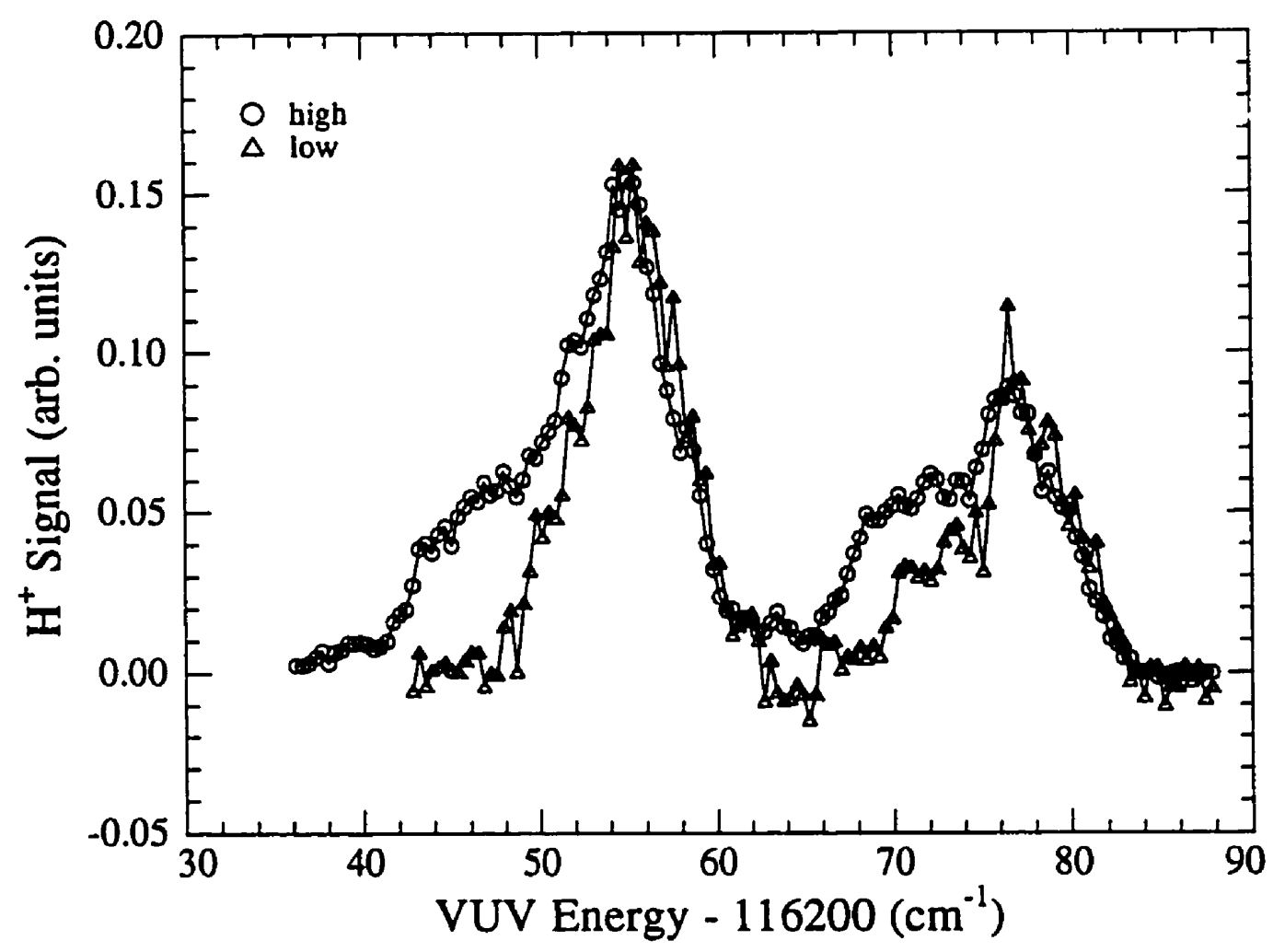

Figure 4.6: $\mathrm{HCl}$ TIPPS spectra taken under different ion-density conditions. The iondensity was varied by changing the VUV fluence by a factor of 6 . The spectra were then normalized by VUV fluence. The discrimination field was $4 \mathrm{~V} / \mathrm{cm}$, pulsed on $0.3 \mu \mathrm{s}$ following photoexcitation. The extraction field was $60 \mathrm{~V} / \mathrm{cm}$, pulsed on $4.1 \mu \mathrm{s}$ after the discrimination field. 
By plotting $E_{1 / 2}$ vs. $\sqrt{F_{d}}$ for the four discrimination fields, and fitting a straight line to these points, it is possible to extrapolate to $F_{d}=0$, to determine $E_{\mathrm{H}^{+}, \mathrm{Cl}^{-}}$. Since the energetic ion-pair dissociation threshold is independent of $J$, the $J=1$ points should simply be shifted to lower energies than the $J=0$ points, by the $J=0, J=1$ energy level difference in the ground vibronic state of $\mathrm{HCl}$. Fig. 4.7 shows the extrapolation to $F_{d}=0$, for the $J=0$ points, together with the $J=1$ points, shifted by the well-known $J=0, J=1$ energy level difference [107].

To improve the precision of the extrapolations, it is desirable to extend the points in Fig. 4.7 to lower discrimination fields. Unfortunately, it was found that the lower discrimination field spectra had blue-edges which were shifted by ion-density. This is presumably due to collisions between the high-v ion-pair states and $\mathrm{HCl}^{+}$ions, breaking the ion-pair states apart in the waiting period. The lower binding energy states of high$v$ are more susceptible to this loss mechanism. These problems can be eliminated by lowering the VUV power, thereby reducing the ion-density. However, this is at the expense of worsened signal. Given the practical constraints of time, $F_{d}=10.2 \mathrm{~V} / \mathrm{cm}$ was the lowest discrimination field possible for extrapolation. The points in Fig. 4.7 were tested to make sure that they did not shift as ion-density was reduced.

The agreement between the $J=0$ and $J=1$ extrapolations is within the error estimates, providing confidence in the extrapolated value: $116289.0 \pm 0.6 \mathrm{~cm}^{-1}$. However, prior to using Eqn. 4.1 to determine $D_{0}(\mathrm{HCl})$, a correction due to the isotopomers of $\mathrm{HCl}$ must be considered.

\subsubsection{Isotopomer Correction}

$\mathrm{HCl}$ has two abundant isotopomers: $\mathrm{H}^{35} \mathrm{Cl}(75 \%)$ and $\mathrm{H}^{37} \mathrm{Cl}(25 \%)$. These will both contribute to the observed TIPPS signal if only $\mathrm{H}^{+}$is detected. Recall that the position of the field-free ion-pair formation threshold is given by:

$$
E_{\mathrm{H}+, \mathrm{Cl}^{-}}=D_{0}(\mathrm{HCl})+I P(\mathrm{H})-E A(\mathrm{Cl})
$$




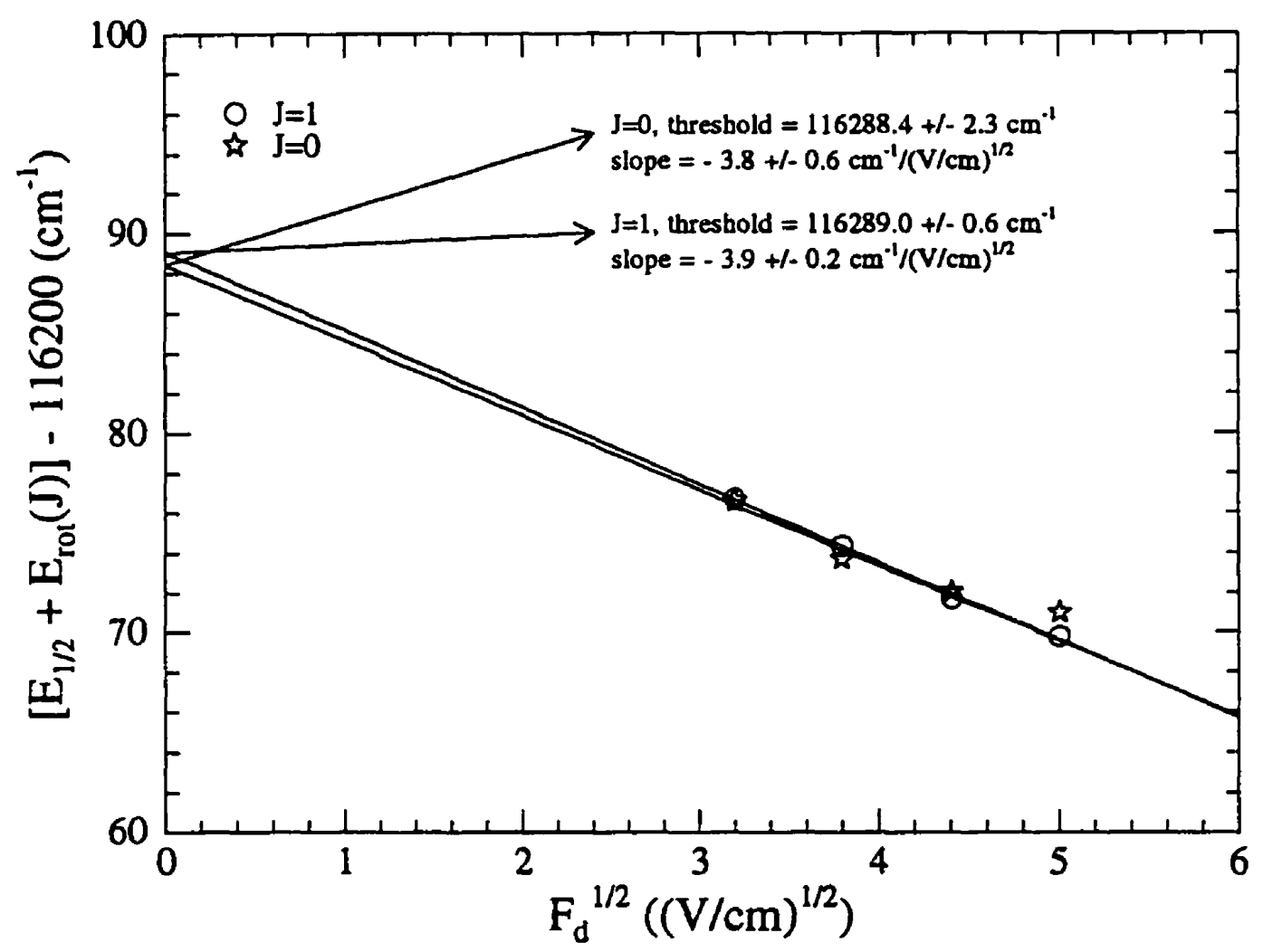

Figure 4.7: Extrapolation to determine the field-free ion-pair formation threshold for $\mathrm{HCl}$. The positions of the blue edges of a series of spectra taken at different discrimination fields are plotted as a function of the square root of the discrimination field. The $J=1$ data has been shifted up in energy by the $J=0, J=1$ energy level difference in the $\mathrm{HCl}$ ground vibronic state $\left(E_{\text {rot }}(0)=0 \mathrm{~cm}^{-1}\right.$ and $\left.E_{\text {rot }}(1)=20.88 \mathrm{~cm}^{-1}\right)$. The extrapolated zero-field thresholds for the two $J$ levels are labeled. The $F=25 \mathrm{~V} / \mathrm{cm}$ point for the $J=0$ extrapolation has been dropped from the fit, as it appears to be anomalous. Dropping this point increases the uncertainty in the $J=0$ extrapolation to zero-field. 
The difference between $E A\left({ }^{35} \mathrm{Cl}\right)$ and $E A\left({ }^{37} \mathrm{Cl}\right)$ is expected to be small $\left(\ll 1 \mathrm{~cm}^{-1}\right)$, however, $D_{0}\left(\mathrm{H}^{35} \mathrm{Cl}\right)$ and $D_{0}\left(\mathrm{H}^{37} \mathrm{Cl}\right)$ do differ by a significant amount, introducing isotopomer specific ion-pair formation thresholds.

The difference between $D_{0}\left(\mathrm{H}^{35} \mathrm{Cl}\right)$ and $D_{0}\left(\mathrm{H}^{37} \mathrm{Cl}\right)$ may be estimated by computing the difference in zero point vibrational energies for these two species, assuming the harmonic oscillator model:

$$
\mathrm{ZPE}\left(\mathrm{H}^{35} \mathrm{Cl}\right)-\mathrm{ZPE}\left(\mathrm{H}^{37} \mathrm{Cl}\right)=\frac{1}{2} \omega_{e}\left(\mathrm{H}^{35} \mathrm{Cl}\right)\left(1-\sqrt{\frac{\mu_{\mathrm{H}^{35} \mathrm{Cl}}}{\mu_{\mathrm{H}^{37} \mathrm{Cl}}}}\right) .
$$

Substituting: $\omega_{e}\left(\mathrm{H}^{35} \mathrm{Cl}\right)=2990.95 \mathrm{~cm}^{-1}[107]$ and $\mu_{\mathrm{H}^{35} \mathrm{Cl}} / \mu_{\mathrm{H}^{37} \mathrm{Cl}}=0.998487$, gives $\mathrm{ZPE}\left(\mathrm{H}^{35} \mathrm{Cl}\right)-\mathrm{ZPE}\left(\mathrm{H}^{37} \mathrm{Cl}\right)=1.13 \mathrm{~cm}^{-1}$. Since $D_{0}=D_{e}-\mathrm{ZPE}$, the difference in the ion-pair thresholds for the two isotopomers is:

$$
E_{\mathrm{H}^{+},{ }^{37} \mathrm{Cl}^{-}}-E_{\mathrm{H}^{+},{ }^{35} \mathrm{Cl}^{-}}=1.13 \mathrm{~cm}^{-1} \text {. }
$$

If TIPPS spectra were collected using $\mathrm{Cl}^{-}$, it would be possible to distinguish between the spectra arising from the two isotopomers by time-of-flight of the $\mathrm{Cl}^{-}$fragments. ${ }^{3}$ The $\mathrm{H}^{37} \mathrm{Cl}$ spectra would be shifted $1.13 \mathrm{~cm}^{-1}$ to the blue, and be one-third the signal strength of the $\mathrm{H}^{35} \mathrm{Cl}$ spectra. However, since all experiments were done using $\mathrm{H}^{+}$, it must now be considered how the composite spectra extrapolations may be corrected to give isotopomer specific thresholds.

Assume that the blue edges of the isotopomer specific TIPPS spectra may be modeled by a signal varying linearly from zero to some constant value - see Fig. 4.8. The blue edges of the composite spectra will then be the sum of two of these structures, weighted by the isotopomer abundances and shifted by the appropriate energies.

For extrapolations to the field-free threshold, the position of the blue edge has been designated as the point at which the signal reaches $50 \%$ of its maximum value $-E_{1 / 2}$. For

\footnotetext{
${ }^{3}$ Discrimination against prompt $\mathrm{Cl}^{-}$fragments would be more difficult than for prompt $\mathrm{H}^{+}$because of the difference in mass. However, since it has been possible to collect MATI spectra of Ar (mass 40) on the apparatus, discrimination against prompt $\mathrm{Cl}^{-}$should be possible. The ${ }^{35} \mathrm{Cl}^{-}$and and ${ }^{37} \mathrm{Cl}^{-}$signals would be easily distinguishable by time-of-flight. Unfortunately, time restrictions prevented this study.
} 


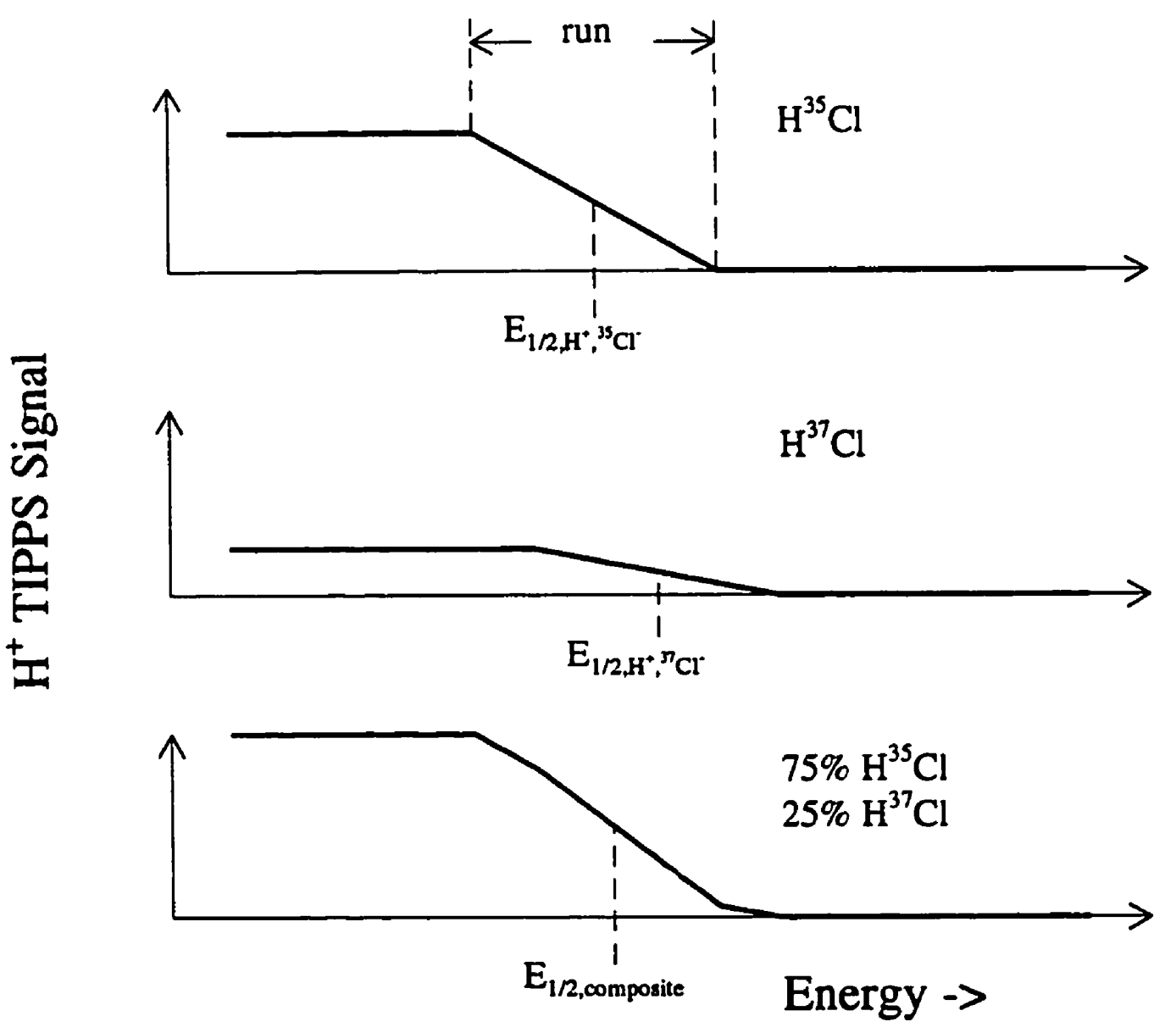

Figure 4.8: Model for the composite blue-edge line-shapes due to the two isotopomers of $\mathrm{HCl}$. 
the composite spectra this position is related, in a systematic manner, to the positions one would find for each specific isotopomer. If this point is at an energy where the signals from both isotopomers are rising linearly, then: ${ }^{4}$

$$
E_{\text {composite }, 1 / 2}=E_{1 / 2, \mathrm{H}^{+},{ }^{35} \mathrm{Cl}^{-}}+0.25\left(E_{\mathrm{H}^{+},{ }^{37} \mathrm{Cl}^{-}}-E_{\mathrm{H}^{+},{ }^{35} \mathrm{Cl}^{-}}\right) \text {. }
$$

This equation allows the thresholds for the composite spectra to be corrected to give isotopomer specific thresholds, since $E_{\mathrm{H}^{+},{ }^{37} \mathrm{Cl}^{-}}-E_{\mathrm{H}+,{ }^{35} \mathrm{Cl}^{-}}$is known from the difference in zero-point energies (see above). Because the correction is the same for all values of $E_{1 / 2, \text { composite }}$ used for the extrapolation, it may simply be applied to the extrapolated field-free ion-pair formation threshold:

$$
E_{\text {composite }}=E_{\mathrm{H}^{+}, 35 \mathrm{Cl}^{-}}+0.25\left(E_{\mathrm{H}^{+}, 37 \mathrm{Cl}^{-}}-E_{\mathrm{H}^{+},{ }^{33} \mathrm{Cl}^{-}}\right)
$$

When the appropriate value is substituted, and the equation rearranged:

$$
E_{\mathrm{H}^{+},{ }^{35} \mathrm{Cl}^{-}}=E_{\text {composite }}-0.28 \mathrm{~cm}^{-1}
$$

Thus, the extrapolated threshold is corrected by this amount to give an isotopomer specific threshold. It is estimated that the error in this correction is unlikely to be greater than $0.1 \mathrm{~cm}^{-1}$.

Combining the uncertainties in the extrapolation, photon energy calibration and isotopomer correction, the uncertainty in the field free ion-pair formation threshold is estimated to be $0.6 \mathrm{~cm}^{-1}$. Thus the minimum energy required to split $\mathrm{H}^{35} \mathrm{Cl}$ from its ground electronic state, $J=0, v=0$ into the ion-pairs $\mathrm{H}^{+}$and ${ }^{35} \mathrm{Cl}^{-}$has been determined to be $116288.7 \pm 0.6 \mathrm{~cm}^{-1}$.

The ionization potential of $\mathrm{H}$ is accurately known [111], and the electron affinity of $\mathrm{Cl}$ has been measured by Trainham et al. [60] to be $29138.3 \pm 0.5 \mathrm{~cm}^{-1}$. When

\footnotetext{
${ }^{4}$ A simple calculation shows that this will be satisfied if the isotopomer specific signals rise from zero to their maximum over more than $1.7 \mathrm{~cm}^{-1}$ (the "run" in Fig. 4.8). This condition is satisfied for all of the $\mathrm{HCl}$ spectra observed.
} 
$I P(\mathrm{H})=109678.8 \mathrm{~cm}^{-1}, E A(\mathrm{Cl})=29138.3 \pm 0.5 \mathrm{~cm}^{-1}$, and $E_{\mathrm{H}^{+}, 35 \mathrm{Cl}^{-}}=116288.7 \pm 0.6$ $\mathrm{cm}^{-1}$ are substituted into Eqn. $1, D_{0}\left(\mathrm{H}^{35} \mathrm{Cl}\right)=35748.2 \pm 0.8 \mathrm{~cm}^{-1}$ is obtained.

\subsubsection{Current Literature Value for $D_{0}(\mathrm{HCl})$}

Most workers who require $D_{0}(\mathrm{HCl})$ quote the value given in Huber and Herzberg's compilation [107]. It is sometimes assumed that this is a spectroscopically determined quantity, however the authors state that it is "from $D_{0}\left(\mathrm{H}_{2}\right), D_{0}\left(\mathrm{Cl}_{2}\right)$ and $\Delta H_{f 0}^{\ominus}(\mathrm{HCl})$ ". While $D_{0}\left(\mathrm{H}_{2}\right)$ and $D_{0}\left(\mathrm{Cl}_{2}\right)$ have been spectroscopically determined, the $\Delta H_{f 0}^{\ominus}(\mathrm{HCl})$ used by Huber and Herzberg is thermochemical in origin. The symbol $\Delta H_{f 0}^{\ominus}(\mathrm{HCl})$ refers to heat absorbed per mole of product formed by the "idealized" reaction

$$
\frac{1}{2} \mathrm{Cl}_{2}(g)+\frac{1}{2} \mathrm{H}_{2}(g) \rightarrow \mathrm{HCl}(g)
$$

occurring at $0 \mathrm{~K}$ and 1 bar pressure. Since a spectroscopically determined $D_{0}(\mathrm{HCl})$ does not appear to be available in the literature, the next few paragraphs will discuss the determination of $D_{0}(\mathrm{HCl})$ using $\Delta H_{f 0}^{\ominus}(\mathrm{HCl})$.

In chemical thermodynamics it is common to define a quantity for each substance known as the "molar enthalpy of formation", or $\Delta H_{f T}^{\ominus}$ (see, for example, Ref. [112] or [113]). This is defined as the enthalpy change for the reaction occurring at temperature $T$ in which one mole of the substance is formed in its standard state from the constituent elements in their standard states. In a "standard state" refers to a being at a pressure of 1 bar, in a manner determined by consistent definition. The JANAF Thermochemical Tables [114], which include tabulated values of $\Delta H_{f T}^{\ominus}$ for many substances, make these conventions clear. For example, for $\mathrm{HCl}$, the standard states of the constituent elements, $\mathrm{H}$ and $\mathrm{Cl}$, are ideal gases of $\mathrm{H}_{2}$ and $\mathrm{Cl}_{2}$ at 1 bar. The standard state of $\mathrm{HCl}$ is also an ideal gas at $I$ bar. For a reaction which occurs with no change in temperature or pressure, the change in enthalpy is the heat absorbed from the surroundings into the system (the change in enthalpy is negative if the reaction liberates heat). Therefore $\Delta H_{\text {fo }}^{\ominus}(\mathrm{HCl})$ refers 
to the heat absorbed in an "idealized" or hypothetical form of the reaction in Eqn. 4.8 at $0 \mathrm{~K}$ and 1 bar. It is idealized, since neither $\mathrm{Cl}_{2}, \mathrm{H}_{2}$ or $\mathrm{HCl}$ behave as ideal gases at 0 $K$ and 1 bar. Although confusing to the uninitiated, the abstraction of standard states is usêtul.

For example, one may discuss the enthalpy of formation of monatomic $\mathrm{Cl}$ from "its elements in their standard states":

$$
\frac{1}{2} \mathrm{Cl}_{2} \rightarrow \mathrm{Cl}
$$

In this case the enthalpy of formation at $0 \mathrm{~K}$ is $\frac{1}{2} D_{0}\left(\mathrm{Cl}_{2}\right)$. Tabulating enthalpies of formation for compounds allows one to compute the changes in enthalpy for more complex reactions. This is sometimes referred to as Hess's law (see for example Ref. [115]):

The overall reaction enthalpy is the sum of the reaction enthalpies of the individual reactions into which a reaction may be divided.

This is simply a statement of conservation of energy.

For example, Eqn. 4.8 may be looked at as the last step in the sequence:

$$
\begin{gathered}
\mathrm{H} \rightarrow \frac{1}{2} \mathrm{H}_{2}, \\
\mathrm{Cl} \rightarrow \frac{1}{2} \mathrm{Cl}_{2}, \\
\frac{1}{2} \mathrm{Cl}_{2}+\frac{1}{2} \mathrm{H}_{2} \rightarrow \mathrm{HCl} .
\end{gathered}
$$

If all substances are assumed to be in their standard states and at $0 \mathrm{~K}$, it is apparent that the overall heat liberated by the three steps will be $D_{0}(\mathrm{HCl})$, which must be equal to the sums of the heats liberated in each individual reaction:

$$
D_{0}(\mathrm{HCl})=\Delta H_{f 0}^{\ominus}(\mathrm{H})+\Delta H_{f 0}^{\ominus}(\mathrm{Cl})-\Delta H_{f 0}^{\ominus}(\mathrm{HCl})
$$

or

$$
D_{0}(\mathrm{HCl})=\frac{1}{2} D_{0}\left(\mathrm{H}_{2}\right)+\frac{1}{2} D_{0}\left(\mathrm{Cl}_{2}\right)-\Delta H_{j 0}^{\ominus}(\mathrm{HCl})
$$


This equation allows the determination of $D_{0}(\mathrm{HCl})$ given the the quantities on the righthand side.

It is clear that $\Delta H_{f 0}^{\ominus}(\mathrm{HCl})$ is an abstraction and cannot be measured even approximately, since neither $\mathrm{Cl}_{2}, \mathrm{H}_{2}$ or $\mathrm{HCl}$ behave as ideal gases at $0 \mathrm{~K}$ and 1 bar. However it can be derived from $\Delta H_{j 298.15 \mathrm{~K}}^{\ominus}(\mathrm{HCl})$, which may be measured. The quantity $\Delta H_{\S 298.15 \mathrm{~K}}^{\ominus}(\mathrm{HCl})$ can be measured since $\mathrm{H}_{2}, \mathrm{Cl}_{2}$ and $\mathrm{HCl}$ are all gases at room temperature and $1 \mathrm{bar}$, and are "ideal enough" to allow one to assume $\Delta H_{j 298.15 \mathrm{~K}}^{\ominus}(\mathrm{HCl}) \approx$ $\Delta H_{\int 298.15 \mathrm{~K}}(\mathrm{HCl})$.

To compute $\Delta H_{f 0}^{\ominus}(\mathrm{HCl})$ from $\Delta H_{f 298.15 \mathrm{~K}}^{\ominus}(\mathrm{HCl})$ one must know the molar changes in enthalpy of $\mathrm{H}_{2}, \mathrm{Cl}_{2}$ and $\mathrm{HCl}$ in their standard states, from $0 \mathrm{~K}$ to $298.15 \mathrm{~K}$. i.e.

$$
\begin{aligned}
\Delta H_{j 0}^{\ominus}(\mathrm{HCl})= & \Delta H_{f 298.15 \mathrm{~K}}^{\ominus}(\mathrm{HCl})-\Delta H_{\mathrm{OK} \rightarrow 298.15 \mathrm{~K}}^{\ominus}(\mathrm{HCl}) \\
& +\frac{1}{2} \Delta H_{0 \mathrm{~K} \rightarrow 298.15 \mathrm{~K}}^{\ominus}\left(\mathrm{H}_{2}\right)+\frac{1}{2} \Delta H_{0 \mathrm{~K} \rightarrow 298.15 \mathrm{~K}}^{\ominus}\left(\mathrm{Cl}_{2}\right)
\end{aligned}
$$

For an isobaric process:

$$
\Delta H_{T_{i} \rightarrow T_{f}}=\int_{T_{i}}^{T_{f}} C_{p}(T) d T
$$

and thus

$$
\Delta H_{\mathrm{OK} \rightarrow 298.15 \mathrm{~K}}^{\ominus}=\int_{0 \mathrm{~K}}^{298.15 \mathrm{~K}} C_{\mathrm{p}}^{\ominus}(T) d T .
$$

Since the standard states are ideal gases their heat capacities $\left(C_{p}^{\ominus}(T)\right.$ 's) may be evaluated from the formulae of statistical mechanics (see the introduction to Ref. [114]). Using Eqn. 4.11 with $C_{p}^{\ominus}(T)$ 's derived from statistical thermodynamics, the JANAF tables list [114]:

$$
\begin{aligned}
\Delta H_{\mathrm{OK} \rightarrow 298.15 \mathrm{~K}}^{\ominus}\left(\mathrm{H}_{2}\right) & =8.467 \mathrm{~kJ} \mathrm{~mol}^{-1} \\
\Delta H_{\mathrm{OK} \rightarrow 298.15 \mathrm{~K}}^{\ominus}\left(\mathrm{Cl}_{2}\right) & =9.181 \mathrm{~kJ} \mathrm{~mol}^{-1} \\
\Delta H_{0 \mathrm{~K} \rightarrow 298.15 \mathrm{~K}}^{\ominus}(\mathrm{HCl}) & =8.640 \mathrm{~kJ} \mathrm{~mol}^{-1}
\end{aligned}
$$

Combining these results:

$$
\Delta H_{f 0}^{\ominus}(\mathrm{HCl})=\Delta H_{f 298.15 \mathrm{~K}}^{\ominus}(\mathrm{HCl})+0.184 \mathrm{~kJ} \mathrm{~mol}^{-1}
$$


Huber and Herzberg [107] do not provide a reference for the value of $\Delta H_{f 0}^{\ominus}(\mathrm{HCl})$ that they used. Finding the source of this value is useful since it will allow critical assessment of the estimated uncertainty in $D_{0}(\mathrm{HCl}) .^{5}$ As shall be seen, the $\Delta H_{j 0}^{\ominus}(\mathrm{HCl})$ used by Huber and Herzberg is almost certainly based on the value of $\Delta H_{j 298.15 \mathrm{~K}}^{\ominus}(\mathrm{HCl})$ determined by Rossini in 1932 [108].

A standard reference for thermochemical data are the critically evaluated JANAF tables [114]. These tables discuss the various determinations of $\Delta H_{f 298.15 \mathrm{~K}}^{\ominus}(\mathrm{HCl})$, and select Rossini's flame calorimetric measurement [108] as the "best" value : $\Delta H_{f 298.15 \mathrm{~K}}^{\ominus}(\mathrm{HCl})=$ $-92.31 \pm 0.21 \mathrm{~kJ} \mathrm{~mol}^{-1}$. Using Eqn. 4.12, $\Delta H_{j 0}^{\ominus}(\mathrm{HCl})=-92.13 \pm 0.21 \mathrm{~kJ} \mathrm{~mol}^{-1} \cdot 6$ In the original paper, Rossini quoted the uncertainty to be $0.05 \mathrm{~kJ} \mathrm{~mol}^{-1}$, which the JANAF editors have downgraded to $0.21 \mathrm{~kJ} \mathrm{~mol}^{-1}$, presumably based on slight discrepancies with subsequent measurements of other groups.

To achieve such high precision Rossini used a sophisticated calorimetric technique, in which $\mathrm{Cl}_{2}$ was reacted with $\mathrm{H}_{2}$ for a period of time. The amount of $\mathrm{HCl}$ produced and the temperature rise of a surrounding water bath were recorded. By heating the same calorimeter electrically using resistive heating, and monitoring the temperature rise, it was possible to correlate absorbed energy with temperature rise, allowing the heat liberated in the reaction

$$
\frac{1}{2} \mathrm{Cl}_{2}(g)+\frac{1}{2} \mathrm{H}_{2}(g) \rightarrow \mathrm{HCl}(g)
$$

to be determined. There are many systematic errors which may plague such an experiment (see the articles in Ref. [116] for supplementary information on the calorimetric technique

${ }^{5}$ Huber and Herzberg [107] write

$$
D_{0}(\mathrm{HCl})=4.433_{6} \mathrm{eV}
$$

where the last digit is subscripted to indicate uncertainty. In their introduction they state that "where the last digit is given as a subscript, we expect that the uncertainty may considerably exceed \pm 10 units of the last decimal place." Applying this rule, the uncertainty in their $D_{0}(\mathrm{HCl})$ is greater than $0.001 \mathrm{eV}$ $\left(8 \mathrm{~cm}^{-1}\right)$.

'The JANAF tables [114] quote the same uncertainty for $\Delta H_{f 298.15 \mathrm{~K}}^{\ominus}(\mathrm{HCl})$ and $\Delta H_{f 0}^{\ominus}(\mathrm{HCl})$, so presumably the correction of Eqn. 4.12 is quite accurate. 
used). However, Rossini's work seems to be very thorough, and after over 50 years the JANAF tables still recommend this value (with the downgraded uncertainty mentioned above).

Substituting the JANAF recommended values of the dissociation energies ${ }^{7}$

$$
\begin{aligned}
& D_{0}\left(\mathrm{Cl}_{2}\right)=19999.1 \pm 1.0 \mathrm{~cm}^{-1} \\
& D_{0}\left(\mathrm{H}_{2}\right)=36118.3 \pm 1.0 \mathrm{~cm}^{-1}
\end{aligned}
$$

together with Rossini's heat of formation corrected to $0 \mathrm{~K}$ [114],

$$
\begin{aligned}
\Delta H_{f 0}^{\ominus}(\mathrm{HCl}) & =-92.128 \pm 0.21 \mathrm{~kJ} \mathrm{~mol}^{-1} \\
& =-7701 \pm 18 \mathrm{~cm}^{-1}
\end{aligned}
$$

into Eqn. 4.9, the dissociation energy of $\mathrm{HCl}$ is obtained:

$$
D_{0}(\mathrm{HCl})=35760 \pm 18 \mathrm{~cm}^{-1} \text {. }
$$

Clearly the estimated error is dominated by the uncertainty in $\Delta H_{f 0}^{\ominus}(\mathrm{HCl})$.

Finally it is noted that $\mathrm{D}_{0}(\mathrm{HCl})$ in Huber and Herzberg [107] is $35759 \mathrm{~cm}^{-1}$. Therefore it is likely that this was derived from Rossini's value of $\Delta H_{j 298.15 \mathrm{~K}}^{\ominus}(\mathrm{HCl})$ and hence, has a comparable error $\left( \pm 18 \mathrm{~cm}^{-1}\right) .^{8}$

The present determination of $D_{0}\left(\mathrm{H}^{35} \mathrm{Cl}\right)=35748.2 \pm 0.8 \mathrm{~cm}^{-1}$, by TIPPS, is within the error bars of the literature value (Eqn. 4.14).

\footnotetext{
${ }^{7}$ More precise determinations of $D_{0}\left(\mathrm{H}_{2}\right)$ and possibly $D_{0}\left(\mathrm{Cl}_{2}\right)$ have been obtained in recent years (see Ref. [51]), however their use is not warranted here since the uncertainty in $\Delta H_{10}^{\ominus}(\mathrm{HCl})$ dominates the uncertainty in $D_{0}(\mathrm{HCl})$.

- An earlier diatomic data compilation due to Herzberg (Table 39 of Ref. [4]) references a set of tables from Rossini as the source for the heat of formation of $\mathrm{HCl}$ used in determining $\mathrm{D}_{0}(\mathrm{HCl})$.
} 


\section{Chapter 5}

\section{Threshold Ion-Pair Spectroscopy of $\mathrm{O}_{2}$ : Observation of Multiple Ion-Pair Dissociation Thresholds}

\subsection{Introduction}

In the case of $\mathrm{HCl}$, the series of peaks observed in the TIPPS spectrum correspond to the differing amounts of initial rotational excitation. In single-photon PFI-ZEKE spectroscopy these are also observed, however, much of the initial interest in PFI-ZEKE spectroscopy was because of the observation of additional energetic thresholds due to the possibility of leaving the ion in different rovibronic states. In particular, access to rotationally resolved cross-sections at threshold was interesting, since traditional photoelectron spectroscopy was unable to provide such information due to inadequate resolution (with the exception of $\mathrm{H}_{2}$ ). It is natural to ask whether or not different dissociation thresholds could be observed in TIPPS spectroscopy - corresponding to excitation of either the positive or negative ion fragments? For $\mathrm{HCl}$, it is not possible for either the $\mathrm{H}^{+}$fragment or 
$\mathrm{Cl}^{-}$fragment to show any internal excitation, since $\mathrm{H}^{+}$is structureless, and $\mathrm{Cl}^{-}$has no excited states below its detachment threshold. However, it is possible to think of other ion-pairs which could show internal excitation. It turns out that $\mathrm{O}_{2}$ provides an excellent example of the observation of a second ion-pair dissociation threshold - as described in this chapter.

In 1975, Dehmer and Chupka published a high-resolution $\left(14 \mathrm{~cm}^{-1}\right)$ study of photoionpair formation in $\mathrm{O}_{2}$ using monochromatized radiation [37]. The first ion-pair dissociation channel open is: $\mathrm{O}_{2}+h \nu \rightarrow \mathrm{O}^{+}\left({ }^{4} S\right)+\mathrm{O}^{-}\left({ }^{2} P_{3 / 2}\right)$. The step-like structure they observed at threshold has been discussed previously in Chapter 1. At an energy of approximately $20 \mathrm{meV}$ above these steps (at $71.69 \mathrm{~nm}$ ), a second sharp rise is observed (see Fig. 5.1). Quoting the authors [37]:

The sharp step at $71.69 \mathrm{~nm}$ may be tentatively assigned to the ion pair dissociation continuum produced from the $J=1$ state of $\mathrm{O}_{2}$ yielding $\mathrm{O}^{-}$in the ${ }^{2} P_{1 / 2}$ state. Such identification leads to a value of the spin-orbit splitting in $\mathrm{O}^{-}$of $22 \mathrm{meV}$, in excellent agreement with the value of $22.4 \pm 0.5 \mathrm{meV}$ reported by Hotop, Patterson and Lineberger. ${ }^{31}$ Such an assignment is far from compelling and the resulting value of the spin-orbit splitting should be viewed with caution.

So although it was not certain that a second threshold corresponding to $\mathrm{O}_{2}+h \nu \rightarrow$ $\mathrm{O}^{+}+\mathrm{O}^{-}\left({ }^{2} P_{1 / 2}\right)$ would be observed, it seemed promising.

\subsection{Experimental}

Excitation to the ion-pair dissociation threshold for $\mathrm{O}_{2}$ requires approximately $17 \mathrm{eV}$ photons. These were generated using resonantly enhanced four-wave sum-mixing in $\mathrm{Kr}\left(2 \nu_{1}+\nu_{2}\right)$. The fixed frequency $\left(\nu_{1}\right)$ was tuned to a two-photon resonance in $\mathrm{Kr}$ 
$\left(2 \nu_{1}=98855.871 \mathrm{~cm}^{-1}[101]\right)$, and $\nu_{2}$ was scanned. The $\nu_{1}$ frequency $(202.3 \mathrm{~nm})$ was not generated by frequency doubling, as the high energy doubling limit in BBO is 204.8 $\mathrm{nm}$ [78]. Instead, it was generated by sum-mixing $606.9 \mathrm{~nm}$ light with $303.5 \mathrm{~nm}$ light in BBO [117]. The $303.5 \mathrm{~nm}$ light was generated by doubling $606.9 \mathrm{~nm}$ (from a dye laser) in KDP. The residual $606.9 \mathrm{~nm}$ from this process, was then sum-mixed with the $303.5 \mathrm{~nm}$ in $\mathrm{BBO}$, to generate the desired frequency. Between the doubling and sum-mixing crystals the $303.5 \mathrm{~nm}$ light was rotated so that it had the same polarization as the 606.9 for the sum-mixing process. Typically $300 \mu \mathrm{J} /$ pulse could be produced. The $\nu_{2}$ frequency was generated by frequency doubling a dye laser operating with Coumarin 480 dye, in BBO.

Pure $\mathrm{O}_{2}$ was used. The stagnation pressure was typically 1 atm, and the pulsed valve had a nozzle diameter of $1 \mathrm{~mm}$. The nozzle was $5.2 \mathrm{~cm}$ away from the VUV interaction region. With the molecular beam on, the average pressure in the chamber was $10^{-5}$ torr.

The discrimination against prompt $\mathrm{O}^{+}$fragments, is more difficult than for $\mathrm{H}^{+}$because of the mass difference. However this did not present a problem, since discrimination against $\mathrm{Ar}^{+}$had already been demonstrated. Typical spectra were collected with a delay time of $4.9 \mu$ s between the start of the discrimination field and the extraction field, and with a discrimination field of $2.1 \mathrm{~V} / \mathrm{cm}$, and an extraction field of $60 \mathrm{~V} / \mathrm{cm}$.

\subsection{Results and Discussion}

Figure 5.1 shows the prompt $\mathrm{O}^{+}$ion-yield in the vicinity of the photoion-pair formation threshold. This is similar to the results presented by Dehmer and Chupka (see Fig.'s 4 and 6 of Ref. [37]). Slight differences due to the temperatures of the samples are expected - and the signal to noise of Fig 5.1 is not as good as in Dehmer and Chupka's work. However, the "step" at $71.69 \mathrm{~nm}$, mentioned in the previous section, is clearly visible near the $\mathrm{O}_{2}(N=1)+h \nu \rightarrow \mathrm{O}^{+}\left({ }^{4} S\right)+\mathrm{O}^{-}\left({ }^{2} P_{1 / 2}\right)$ ion-pair threshold.

A TIPPS spectrum covering the threshold region is shown in Fig. 5.2 (the experimen- 


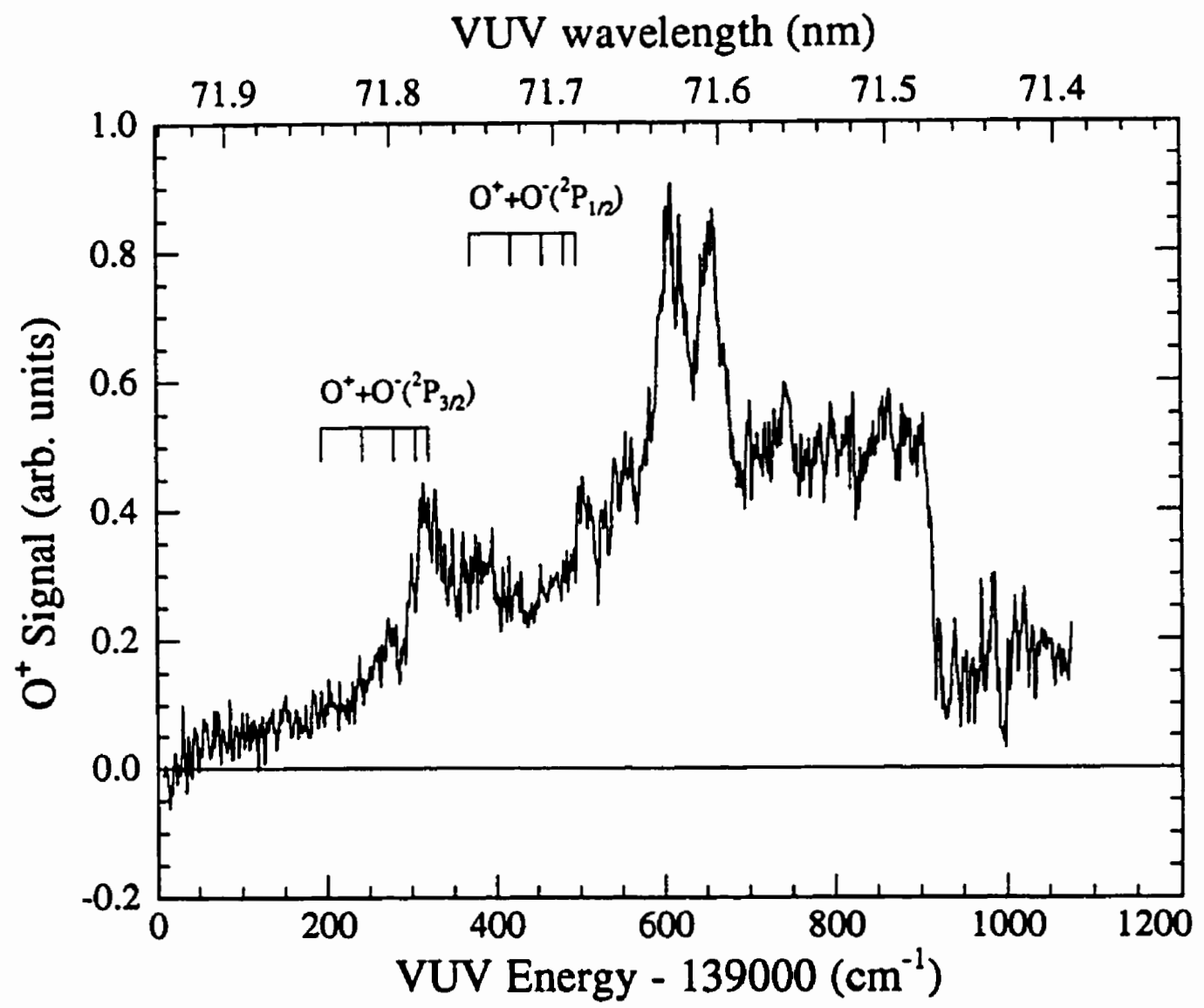

Figure 5.1: Prompt photoion-pair yield from $\mathrm{O}_{2}$. An extraction field of $60 \mathrm{~V} / \mathrm{cm}$ was pulsed on $0.2 \mu \mathrm{s}$ following photoexcitation. No discrimination field was used. The field-free ion-pair formation thresholds for $\mathrm{O}_{2}(N=1,3,5,7,9)+h \nu \rightarrow \mathrm{O}^{+}\left({ }^{4} S\right)+$ $\mathrm{O}^{-}\left({ }^{2} P_{3 / 2},{ }^{2} P_{1 / 2}\right)$ have been marked (see text). 


\begin{tabular}{|c|c|c|c|}
\hline \hline$N$ & $\begin{array}{c}F_{1}(J=N+1) \\
\left(\mathrm{cm}^{-1}\right)\end{array}$ & $\begin{array}{c}F_{2}(J=N) \\
\left(\mathrm{cm}^{-1}\right)\end{array}$ & $\begin{array}{c}F_{3}(J=N-1) \\
\left(\mathrm{cm}^{-1}\right)\end{array}$ \\
\hline 1 & 1.00 & 2.88 & -1.09 \\
3 & 15.30 & 17.25 & 15.17 \\
5 & 41.14 & 43.13 & 41.11 \\
7 & 78.48 & 80.49 & 78.52 \\
9 & 127.31 & 129.35 & 127.41 \\
\hline
\end{tabular}

Table 5.1: Rotational energy levels of $\mathrm{O}_{2}$ from Table 10 of Amiot and Verges [118]. The energy origin is the hypothetical level, $N=J=0$. The original table lists the values more precisely and to higher $N$.

tal conditions are listed in the caption). This spectrum is qualitatively similar to that presented for $\mathrm{HCl}$ (Fig. 4.2). However, in this case, the field-free thresholds are known reasonably accurately a priori. These have been marked on the figure - computed using:

$$
\begin{aligned}
E_{\mathrm{O}+\mathrm{O}-}(N, J)= & D_{0}\left(\mathrm{O}_{2}\right)+I P(\mathrm{O})-E A(\mathrm{O}) \\
& -\left[E_{\text {rot }}(N, J)-E_{\text {rot }}(N=1, J=0)\right]
\end{aligned}
$$

$E_{\text {rot }}(N, J)$ refers to the rotational energy of $\mathrm{O}_{2}$ in its ground electronic state $X^{3} \Sigma_{g}^{-}$. The rotational energy level structure is slightly more complicated than for $\mathrm{HCl}$, since the levels are now characterized by two quantum numbers: $N$ and $J$, where $J=N$ or $J=N \pm 1$. This is due to the coupling of the electron spin $(S=1)$ and normal mechanical angular momentum $N$, to make the total angular momentum $J$. These energy levels, as determined by Amiot and Verges [118], are listed in Table 5.1. The reader will note that even values of $N$ are not listed, as they are prohibited by nuclear spin statistics (see, for example, Ref. [119]).

After a review of various determinations of $D_{0}\left(\mathrm{O}_{2}\right)$, Cosby and Huestis [120] recommend $D_{0}\left(\mathrm{O}_{2}\right)=41268.6 \pm 1.1 \mathrm{~cm}^{-1}$ (referenced from the $N=1, J=0$ level of the ground electronic state).

The ionization potential of the oxygen atom $I P(0)$ is accurately known [101]: $I P(0)=$ 


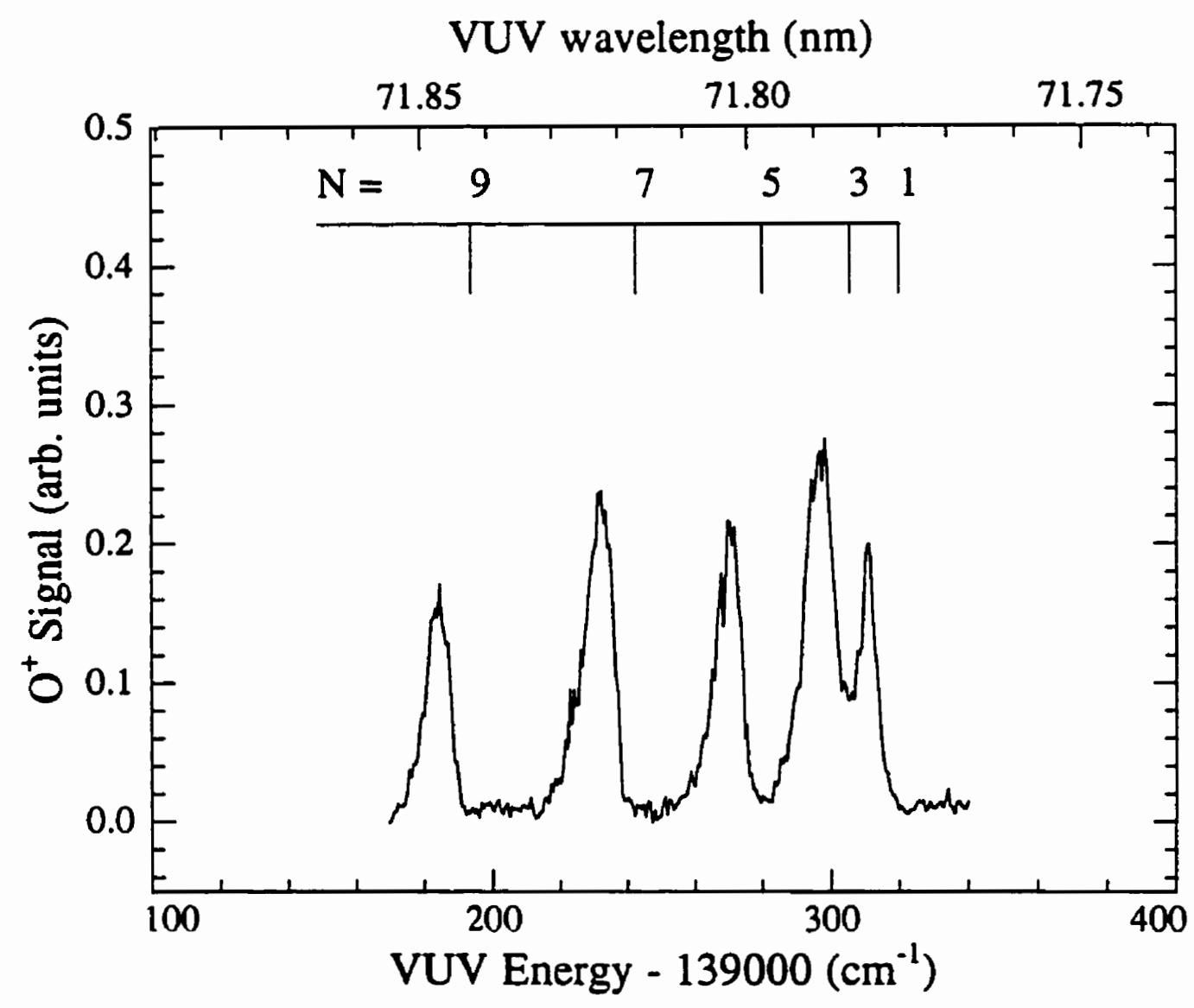

Figure 5.2: TIPPS spectrum of $\mathrm{O}_{2}$. The discrimination field was $2.1 \mathrm{~V} / \mathrm{cm}$, pulsed on 0.3 $\mu \mathrm{s}$ after photoexcitation. An extraction field of $60 \mathrm{~V} / \mathrm{cm}$ was pulsed on $4.9 \mu \mathrm{s}$ later. The field-free ion-pair formation thresholds for $\mathrm{O}_{2}(N, J=N+1)+h \nu \rightarrow \mathrm{O}^{+}\left({ }^{4} S\right)+\mathrm{O}^{-}\left({ }^{2} P_{3 / 2}\right)$ have been marked (see text for details). 
$109837.02 \pm 0.06 \mathrm{~cm}^{-1}$

The final quantity required to use Eqn. 5.1 is the electron affinity of the oxygen atom $E A(O)$. This was determined precisely by Neumark and co-workers [121], to a relative precision of $0.3 \mathrm{ppm}$ [52]. They found:1 $E A(O)=11784.648 \pm 0.006 \mathrm{~cm}^{-1}$.

Each peak in Fig. 5.2 clearly lies below its respective threshold computed using Eqn. 5.1, with the values discussed above. This confirms that the signal observed originates from the electric field induced dissociation of high- $v$ states lying just below the dissociation limit. Indeed, when the magnitude of the discrimination field is varied, the blue edge of each peak shifts to lower energy, as more of the higher- $v$ ion-pair states are depleted in the waiting period. This systematic shift of the blue edge may be used to extrapolate to zero discrimination field, in a similar manner to Fig. 4.7 (the extrapolation is slightly complicated by the fact that each peak is the composite of the F1, F2 and F3 levels). By rearranging Eqn. 5.1, $D_{0}\left(\mathrm{O}_{2}\right)$ may then be determined. However, this is not too useful, since $D_{0}\left(\mathrm{O}_{2}\right)$ is already known to high precision (except perhaps as a confirmation of the procedure).

As part of their accurate determination of $E A(0)$, Neumark and co-workers [121] also determined the splitting between the ground-state $\left({ }^{2} P_{3 / 2}\right)$ and excited state $\left({ }^{2} P_{1 / 2}\right)$ spinorbit components of $\mathrm{O}^{-}$. They found: $E\left({ }^{2} P_{1 / 2}\right)-E\left({ }^{2} P_{3 / 2}\right)=177.13 \pm 0.05 \mathrm{~cm}^{-1}$. Thus, a second set of energetic thresholds corresponding to the thresholds for $\mathrm{O}_{2}(N)+h \nu \rightarrow$ $\mathrm{O}^{+}\left({ }^{4} S\right)+\mathrm{O}^{-}\left({ }^{2} P_{1 / 2}\right)$ was expected - shifted by exactly $177 \mathrm{~cm}^{-1}$ from the first set (those in Fig. 5.2). When this energy range is scanned, using identical conditions to those of Fig. 5.2, a second set of displaced peaks is observed (Fig. 5.3). To clarify, the TIPPS signal observed in the energy range, $139320 \mathrm{~cm}^{-1}$ to $139500 \mathrm{~cm}^{-1}$, corresponds to the electric-field induced dissociation of high- $v$ ion-pair states, where the $\mathrm{O}^{-}$partner is in the excited spin-orbit state $\left({ }^{2} P_{1 / 2}\right)$.

This is an exciting result, which suggests several possible future lines of research.

\footnotetext{
'This is a slightly corrected value discussed in Blondel's review article [52].
} 


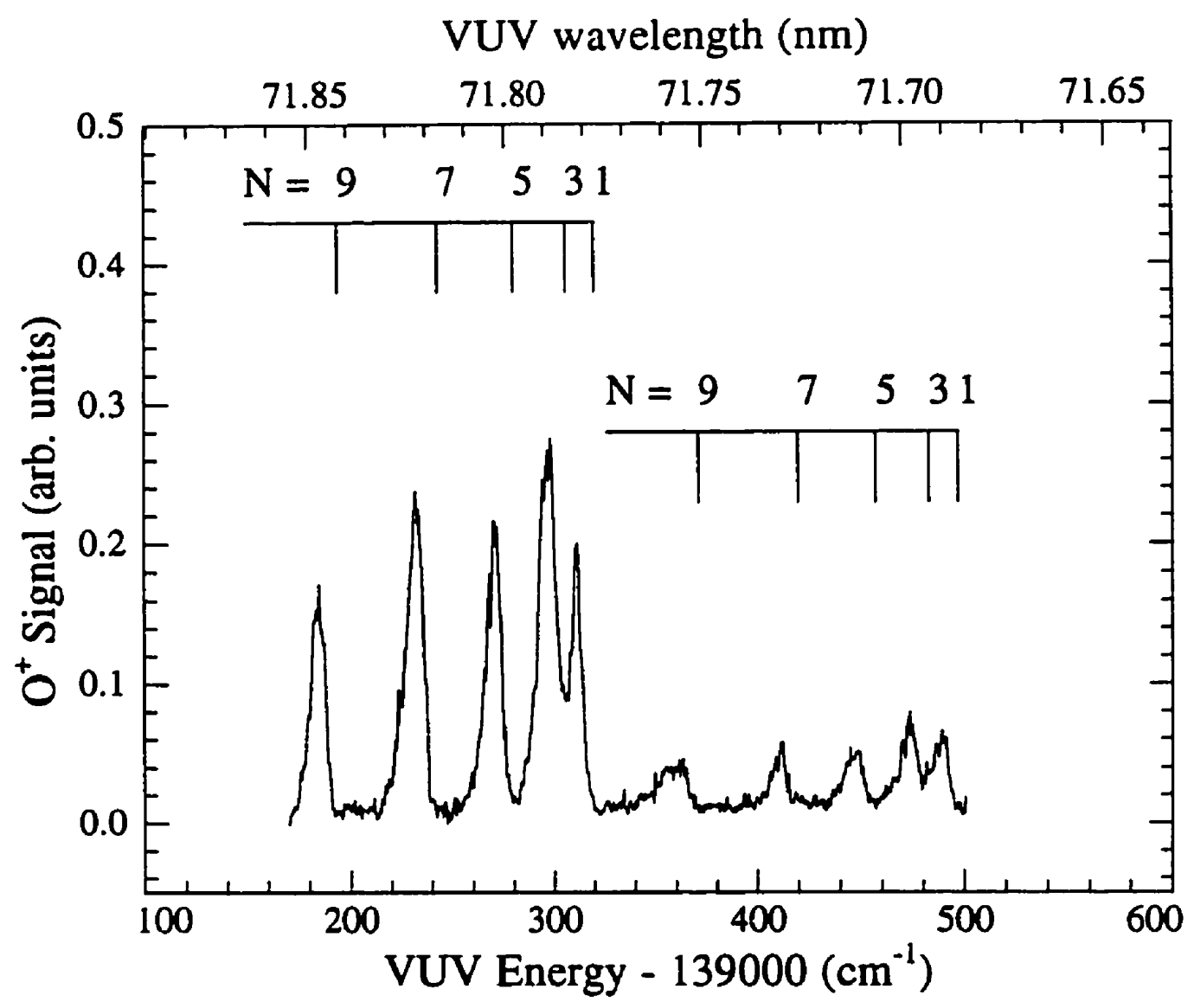

Figure 5.3: TIPPS spectrum of $\mathrm{O}_{2}$. The discrimination field was $2.1 \mathrm{~V} / \mathrm{cm}$, pulsed on $0.3 \mu \mathrm{s}$ after photoexcitation. An extraction field of $60 \mathrm{~V} / \mathrm{cm}$ was pulsed on $4.9 \mu \mathrm{s}$ later. The field-free ion-pair formation thresholds for $\mathrm{O}_{2}(N)+h \nu \rightarrow \mathrm{O}^{+}\left({ }^{4} S\right)+\mathrm{O}^{-}\left({ }^{2} P_{3 / 2},{ }^{2} P_{1 / 2}\right)$ have been marked (see text for details). 
If the $\mathrm{O}^{-}$spin-orbit splitting was not known a priori, the TIPPS spectrum shown in Fig. 5.3 could provide this value. It is the energy separation between the two sets of peaks. This suggests that the TIPPS technique could be used for negative-ion spectroscopy - some of which might be difficult otherwise. For example, acetylene $\mathrm{C}_{2} \mathrm{H}_{2}$ has been demonstrated to produce ion-pairs [47]: $\mathrm{C}_{2} \mathrm{H}_{2}+h \nu \rightarrow \mathrm{C}_{2} \mathrm{H}^{-}+\mathrm{H}^{+}$. There will be different energetic thresholds for this process, depending on the amount of excitation left in the $\mathrm{C}_{2} \mathrm{H}^{-}$fragment. Although one could not hope (presently) to distinguish the rotational thresholds, a TIPPS spectrum should show the vibrational levels of $\mathrm{C}_{2} \mathrm{H}^{-}-$which are otherwise difficult to obtain.

This raises an interesting point concerning the decay of highly vibrationally excited ion-pair states. In the case of $\mathrm{O}_{2}$, at the second set of peaks $\left({ }^{2} P_{1 / 2}\right)$ the ion-pairs have an additional predissociation channel open for decay: $\mathrm{O}^{+}+\mathrm{O}^{-}\left({ }^{2} P_{3 / 2}\right)$. Classically speaking, the $\mathrm{O}^{+}$and $\mathrm{O}^{-}$ions can collide, the $\mathrm{O}^{-}$ion relax to the lower spin-orbit state, the energy goes into translation, and thus the two ions escape from one another. This is analogous to autoionization loss for Rydberg states. Since the signals observed for the ${ }^{2} P_{1 / 2}$ and ${ }^{2} P_{3 / 2}$ set of peaks are roughly comparable, any loss due to this mechanism must be relatively minor. However, this may not be the case for other high-v ion-pair states. For a $\mathrm{C}_{2} \mathrm{H}^{-}-\mathrm{H}^{+}$ion-pair system, transfer of vibrational energy from the $\mathrm{C}_{2} \mathrm{H}^{-}$ to $\mathrm{H}^{+}$might be quite efficient, introducing a loss mechanism for the high- $v$ ion-pair states, preventing thresholds where $\mathrm{C}_{2} \mathrm{H}^{-}$is vibrationally excited, from being observed. Because of the complexity of this system (and the role external perturbations may play), only experimental work is likely to resolve this question.

An interesting (but difficult) application of the TIPPS technique would be to the study of the reactions of state-selected negative ions. How would this work? Recall that when at the energies corresponding to the second set of TIPPS peaks, only $\mathrm{O}^{+}$ions corresponding to the electric-field induced dissociation of $\mathrm{O}^{-}\left({ }^{2} P_{1 / 2}\right)-\mathrm{O}^{+}$ion-pairs are traveling up the flight tube (see Fig. 2.1). By simply reversing the polarities of all field discrimination and extraction pulses, $\mathrm{O}^{-}$ions could be detected instead $\mathrm{O}^{+}$ions. Then the state of the $\mathrm{O}^{-}$ 
ion drawn up the tube could be changed, by simply changing the VUV wavelength to a TIPPS peak between $139160 \mathrm{~cm}^{-1}$ and $139320 \mathrm{~cm}^{-1}$ (for $\mathrm{O}^{-}\left({ }^{2} P_{3 / 2}\right.$ ), or between 139320 $\mathrm{cm}^{-1}$ and $139500 \mathrm{~cm}^{-1}$ (for $\mathrm{O}^{-}\left({ }^{2} P_{1 / 2}\right)$ ). This "state-selected" beam of $\mathrm{O}^{-}$ions could be sent through a target, and the differential reactivity of the two spin-orbit states of $\mathrm{O}^{-}$ examined. ${ }^{2}$

An example of a state-selected reaction study using positive ions has been presented by Dutuit et al. [124]. In this case, the charge exchange reaction:

$$
\mathrm{Ar}^{+}\left({ }^{2} P_{3 / 2},{ }^{2} P_{1 / 2}\right)+\mathrm{O}_{2} \rightarrow \mathrm{Ar}+\mathrm{O}_{2}^{+}
$$

was studied for the two possible $\mathrm{Ar}^{+}$states: ${ }^{2} P_{1 / 2}$ or ${ }^{2} P_{3 / 2}$, as a function of center-ofmass collision energy. At lower energies $(<0.15 \mathrm{eV})$, no differences in the charge exchange cross-sections are observed, but at $0.6 \mathrm{eV}$ the cross-sections for the two different spin-orbit states differ by a factor of 3 . Perhaps analogous behaviour - strong state dependency of certain reaction cross-sections - could be observed for state-selected negative ions. Stateselected cations (like $\mathrm{Ar}^{+}$) are produced by photoionization of the parent neutral ( $\mathrm{Ar}$ ). Obviously anions cannot be produced in this fashion, and so, because of the difficulties in producing them, no study of the reactions of state-selected negative ions have ever been made [125].

\footnotetext{
${ }^{2}$ This technique is similar to that presented by Merkt et al. [122] and Mackenzie and Softley [123] for producing ions in specific rovibronic states using MATI.
} 


\section{Chapter 6}

\section{Threshold Ion-Pair Spectroscopy of $\mathbf{H F}$}

\subsection{Introduction}

Previous workers have shown that $\mathrm{HF}$ has an abnormally large cross-section for singlephoton ion-pair production ( $\mathrm{HF}+h \nu \rightarrow \mathrm{H}^{+}+\mathrm{F}^{-}$), particularly near the energetic threshold (see Table I of Ref. [34]). Therefore, HF seemed like a natural candidate for initial TIPPS studies. The dissociation energy of $H F$ is reasonably well known $\left( \pm 8 \mathrm{~cm}^{-1}\right)[126]$. Therefore the determination of the dissociation energy, in an analogous manner to that presented for $\mathrm{HCl}$ in Chapter 4, provides a confirmation of the extrapolation procedure. In addition, it has been difficult to assign the Rydberg states responsible for predissociation into ion-pairs, with the low-resolution studies done thus far $\left(\geq 100 \mathrm{~cm}^{-1}\right)$ [64]. Thus, just studying the photoion-pair yield spectra near threshold seemed useful.

In 1969, Dibeler and co-workers [127] characterized photoion-pair formation in HF from $78.5 \mathrm{~nm}$ to $77.5 \mathrm{~nm}$, at a photon energy resolution of $0.1 \mathrm{~nm}\left(\right.$ or $160 \mathrm{~cm}^{-1}$ ). This was followed by a similar study by Berkowitz and co-workers in 1971 with better signal 
to noise, and at slightly higher resolution $\left(0.08 \mathrm{~nm}\right.$, or $\left.130 \mathrm{~cm}^{-1}\right)$ [42]. Immediately following this was an article by Chupka and Berkowitz [31] entitled "Kinetic Energy of Ions Produced by Photoionization of $\mathrm{HF}$ and $\mathrm{F}_{2}$ ". In their paper, the $\mathrm{H}^{+}$emitted by photoionpair formation was kinetic energy analyzed at several different wavelengths. This allowed them to conclude that their measured ion-pair formation threshold from the photoionpair yield spectrum was reasonable. (There was considerable disagreement with Dibeler et al.'s [127] previous work concerning the value of $D_{0}(\mathrm{HF})$, and their measurements provided additional evidence that the value presented in Berkowitz et al. [42], was the correct one. The conclusions are supported by more recent work, including the results presented in this chapter.)

The most recent study of photoion-pair formation in HF has been that of Yencha and co-workers [64]. They measured the photoion-yield with a slightly improved photon resolution $\left(0.06 \mathrm{~nm}, 100 \mathrm{~cm}^{-1}\right)$. However more importantly, extensive theoretical modeling of the cross-section was done, in an attempt to understand which Rydberg states predissociate into ion-pairs, and the predissociation mechanism. This modeling, done by Léfêbvre-Brion and Keller (Orsay), is similar to that done for $\mathrm{HCl}$ by the same workers - discussed in Section 4.2. However, there are significant differences in the conclusions. Recall that for $\mathrm{HCl}$, Rydberg states with a $X^{2} \Pi$ core were inaccessible due to Franck-Condon factors. In the case of HF, the ion-pair threshold is much closer to the first ionization threshold (leaving $\mathrm{HF}^{+}$in the $X^{2} \Pi$ state). Thus it is energetically possible to excite Rydberg states which have low- $v X^{2} \Pi$ cores. These low- $v$ states are Franck-Condon accessible (for the case of $\mathrm{HCl}$, it is only energetically possible to excite Rydberg states with very high- $v X^{2} \Pi$ ion cores, which are not then Franck-Condon factor accessible). Consequently, excitation to Rydberg states with $X^{2} \Pi$ cores contributes to the photoion-yield, in addition to the Rydberg states with $A^{2} \Sigma$ ionic cores (which feature exclusively in the $\mathrm{HCl}$ case). As shall be seen, when examined at high resolution, the photoion-pair yield spectrum of HF differs significantly from that of $\mathrm{HCl}$. 


\subsection{Experimental}

To excite HF from its ground state to the ion-pair formation threshold requires 16.1 $\mathrm{eV}$ photons. Since this is close to the $15.9 \mathrm{eV}$ required for the Ar MATI experiments described in Chapter 3, the same optical setup was used (see Section 3.2).

Since $\mathrm{H}^{+}$was detected in both the $\mathrm{HCl}$ and $\mathrm{HF}$ TIPPS experiments, the discrimination requirements and experimental timings were virtually identical. As with $\mathrm{HCl}$, the delay between the light pulse and extraction field for the TIPPS spectra was typically $4.4 \mu \mathrm{s}$. With this time period discrimination fields as low as $1.5 \mathrm{~V} / \mathrm{cm}$ could be used without significant prompt-ion contamination. To compensate for the movement of the excited states along the beam axis during the discrimination time period, the spectrometer was translated along the molecular beam axis by $0.6 \mathrm{~cm}$.

The HF gas was obtained from a lecture bottle without a regulator. The pressure was controlled by surrounding the lecture bottle with an ice slush bath at $273 \mathrm{~K}$. The vapor pressure of $\mathrm{HF}$ at $273 \mathrm{~K}$, is roughly $0.5 \mathrm{~atm}$ (CRC Handbook). The pure HF gas was expanded from a $1 \mathrm{~mm}$ diameter nozzle. It traveled $5.2 \mathrm{~cm}$ before interacting with the VUV beam. With the molecular beam on, the average main chamber pressure was $1 \times 10^{-5}$ torr.

\subsection{Results and Discussion}

Figure 6.1 shows the prompt photoion-pair yield spectrum for the process: $\mathrm{HF}+h \nu \rightarrow$ $\mathrm{H}^{+}+\mathrm{F}^{-}$in the vicinity of the threshold region. This spectrum has similar artifacts to those discussed for the $\mathrm{HCl}$ spectrum in Section 4.4. Namely, collection efficiency decreases above threshold, and the extraction field may field-dissociate high-v ion-pair states, allowing them to contribute to the "free" photoion-pair yield. Nevertheless, the spectrum should be representative of the prompt yield, and illustrates several important points. 

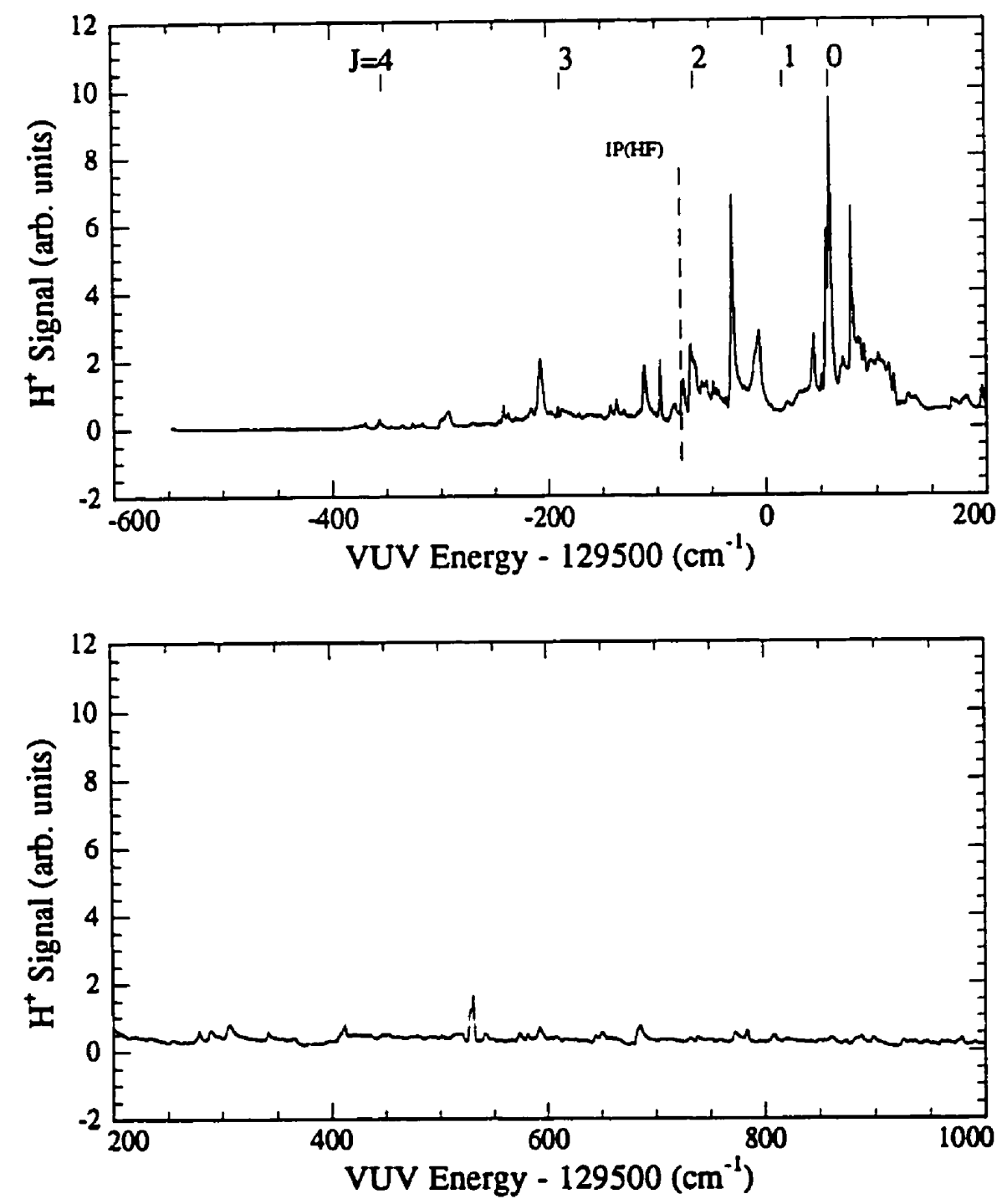

Figure 6.1: Prompt photoion-pair yield from HF. An extraction field of $60 \mathrm{~V} / \mathrm{cm}$ was pulsed on $0.2 \mu \mathrm{s}$ following photoexcitation. No discrimination field was used. No attempt was made to ensure $100 \%$ collection efficiency for fast $\mathrm{H}^{+}$. The field-free ion-pair formation thresholds for each initial $J$ level have been labeled (the determination of these is described in Section 6.4.2). The first ionization threshold of HF [128] has also been marked (IP(HF)). 
In contrast to the prompt photoion-pair yield spectra observed for both $\mathrm{HCl}$ and $\mathrm{O}_{2}$, the HF spectrum shows sharp resonances, with little pseudo-continuum. It should be possible to assign the resonances to specific Rydberg states. This analysis is still at a preliminary stage. Again, as with $\mathrm{HCl}$ it is impossible to identify the energetic threshold for ion-pair formation from the photoion-pair yield, as no step-like structure is observable - unlike for $\mathrm{O}_{2}$ (see Section 1.5.1).

Figure 6.2 shows a TIPPS spectrum of HF which covers the threshold region. The sharp peaks which were in the prompt ion-pair yield, now manifest themselves in the TIPPS spectra. Instead of a single peak for each $J$ level, as with $\mathrm{HCl}$, each TIPPS line (corresponding to an initial $J$ ), consists of multiple resonances. However, the energy range over which signal may be seen is still dependent on the strengths of the electric fields employed (discrimination and extraction). The signal now varies significantly within the energy range dictated by those fields.

Also illustrated in Fig. 6.2 is the theoretical population distribution of a $300 \mathrm{~K}$ sample, over the initial $J$ states. As the HF will be cooled by the supersonic expansion (this is difficult to quantify), $300 \mathrm{~K}$ represents an upper bound on the temperature of the distribution. There appears to be more TIPPS signal from high- $J$ levels compared to low$J$ levels, based on the initial population distribution. It is not expected that excitation to the initial Rydberg states could be this $J$-dependent, thus another explanation must be sought. A $J$-dependent predissociation automatically suggests that the "gyroscopic" perturbation may be responsible for the predissociation of the Rydberg states into ionpairs (see for example Ref. [110]). This has been discussed by Berkowitz and co-workers [129] to explain a similar observation in the photoion-pair yield of $\mathrm{Cl}_{2}$. However, in the case of HF there are alternative explanations: the high- $J$ enhancement could be associated with an increase in the density of Rydberg states (energy spacings), or changes in the efficiency of processes competing for fate of these Rydberg states. Perhaps autoionization becomes less competitive as $J$ increases. Without an assignment of the Rydberg states, it is difficult to come to any conclusion concerning the high- $J$ enhancement, or the nature 


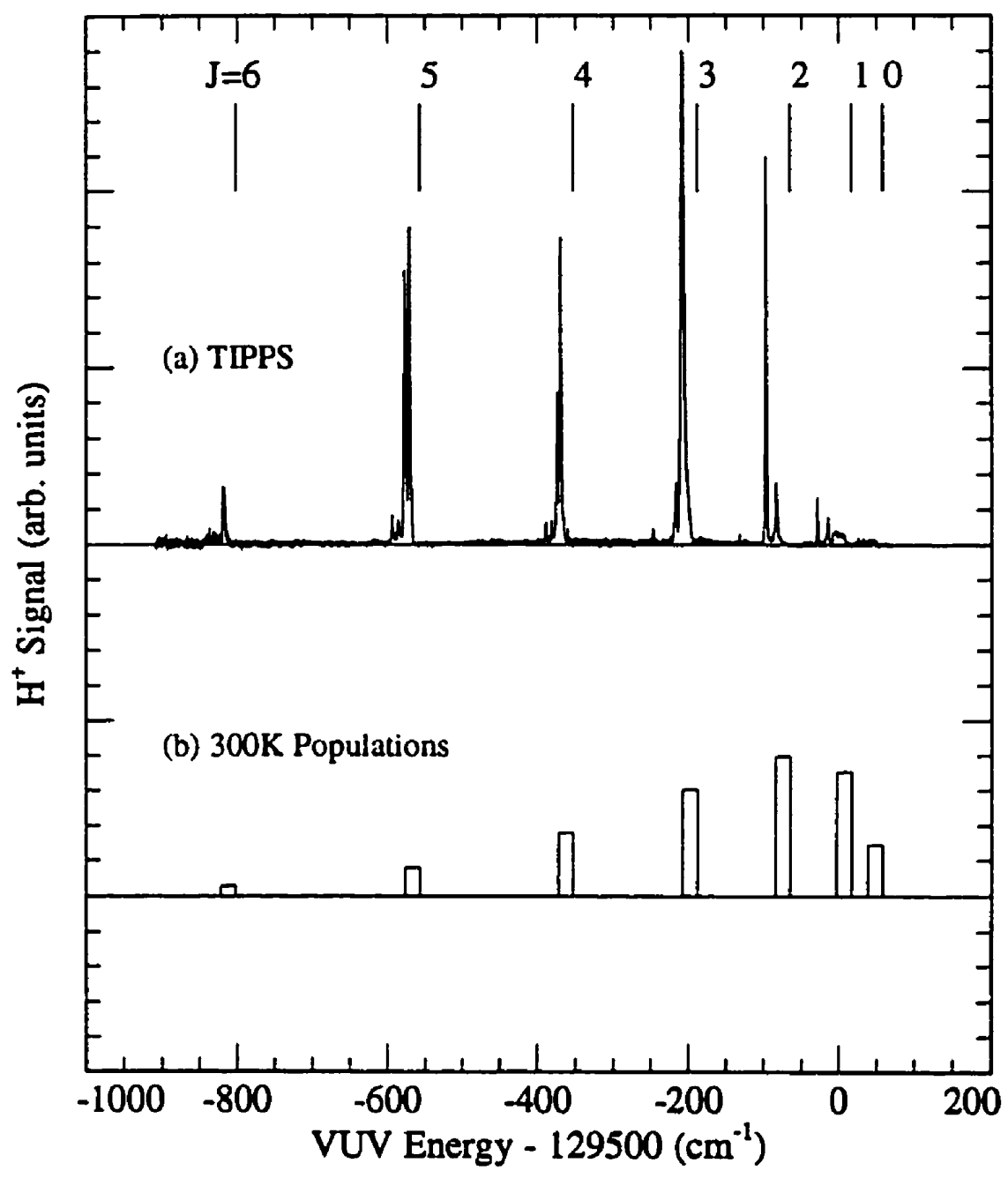

Figure 6.2: (a) TIPPS spectrum of HF. The discrimination field was $3 \mathrm{~V} / \mathrm{cm}$, pulsed on $300 \mathrm{~ns}$ following photoexcitation. The extraction field was $60 \mathrm{~V} / \mathrm{cm}$, pulsed on $3.4 \mu \mathrm{s}$ after photoexcitation. (b) The relative populations of the different initial $J$ states of HF at $300 \mathrm{~K}$. 
of the predissociation responsible for the ion-pairs.

Figures 6.3 through to 6.7 , show each individual TIPPS line in more detail together with the corresponding prompt ion yield (Fig. 6.1) over the same energy range. Since the prompt-ion signal is simply the TIPPS signal without a discrimination field, resonances which appear in the TIPPS spectra should also appear in the prompt ion-yield spectra (as discussed previously, this is an artifact due to the fact that the extraction field will field-dissociate high- $v$ ion-pair states). Naturally, the opposite is not true - resonances which appear in the prompt ion-yield do not necessarily show up in the TIPPS spectra.

TIPPS spectra may be helpful in assigning resonances in the photoion-yield spectra, as they indicate the initial $J$ of resonances. All signal within a specific TIPPS line must correspond to excitation from a specific initial $J$ state. ${ }^{1}$ This will be illustrated by an example.

Consider excitation to the resonance labeled with the * in Fig. 6.3. This resonance should correspond to excitation from an initial state $J=0$. Therefore it is reasonable to look for transitions from other initial $J$ levels to the same final state. Simply add or subtract the appropriate differences in ground state rotational energy levels to find out where these should be. Indeed, transitions to the same final energy from $J=1$ and $J=2$ are observed in Fig.'s 6.4 and 6.5 (also labeled with *). Therefore, since it is single-photon excitation, it is possible to conciude that the final state is $J=1$ ( $\Delta J=0, \pm 1$ for single-photon transitions). Unfortunately, there do not appear to be any other resonances for which this explanation works. However, this single assignment of $J$ to a specific resonance, will be useful in checking the assignment of Rydberg state structure to the photoion-yield spectrum.

\footnotetext{
${ }^{1}$ If the signal originated from a higher $J$ state, excitation would be to an energy above the ionpair threshold, thus the molecule would not survive the waiting period prior to electric-field induced dissociation. If the signal was due to a lower $J$ state, it would not be possible to dissociate it with the electric field.
} 


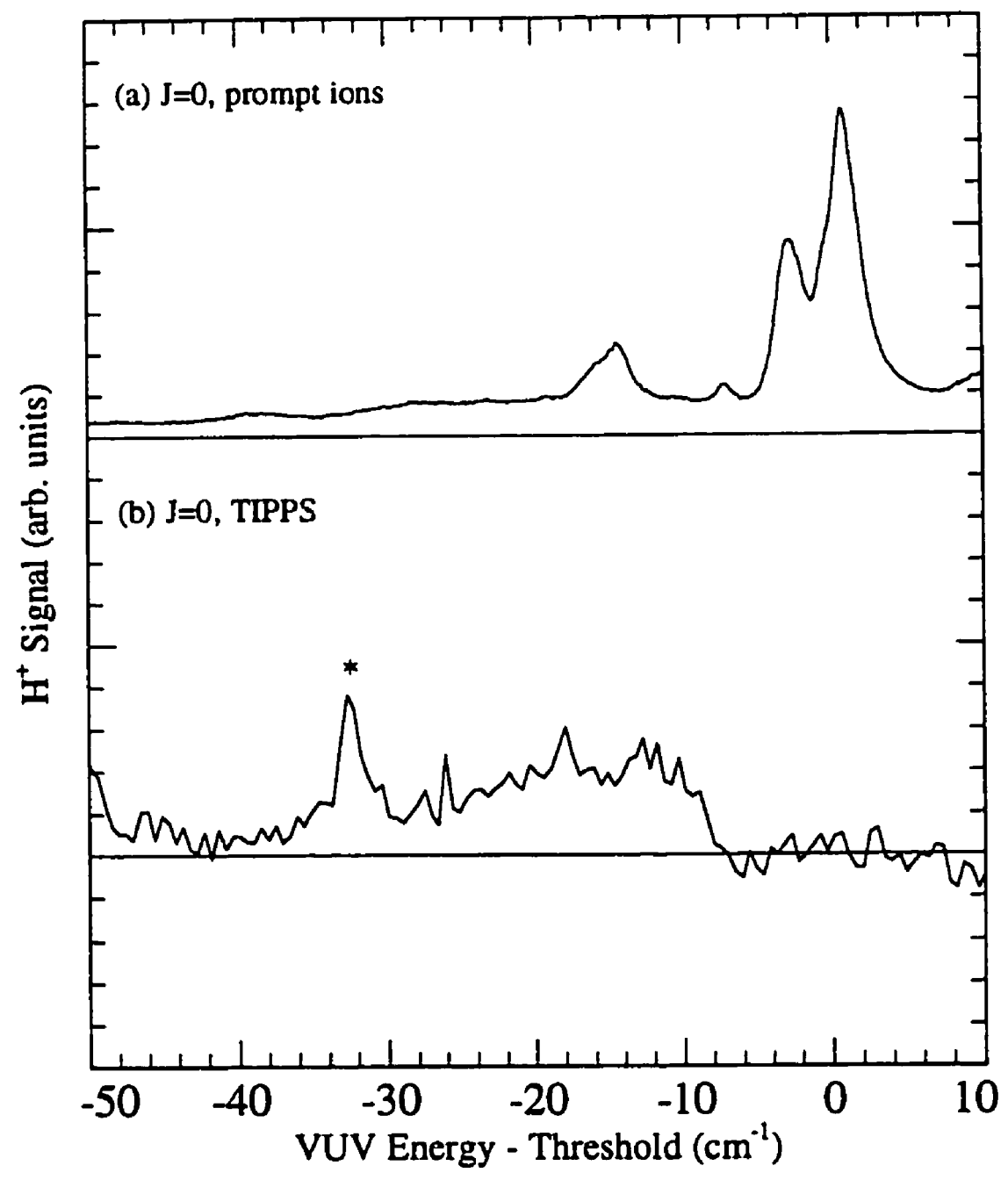

Figure 6.3: (a) $\mathrm{H}^{+}$prompt ion yield and (b) TIPPS spectrum of $\mathrm{HF}$ at the $J=0$ threshold. The energy scale is relative to the $J=0$ field-free ion-pair formation threshold. For the prompt-ion yield spectrum, a $60 \mathrm{~V} / \mathrm{cm}$ extraction field was pulsed on $0.2 \mu \mathrm{s}$ following photoexcitation. The TIPPS spectrum was collected with a discrimination field of $3 \mathrm{~V} / \mathrm{cm}$, pulsed on 300 ns following photoexcitation, and an extraction field of 60 $V / \mathrm{cm}$, pulsed on $3.4 \mu$ s after photoexcitation. 


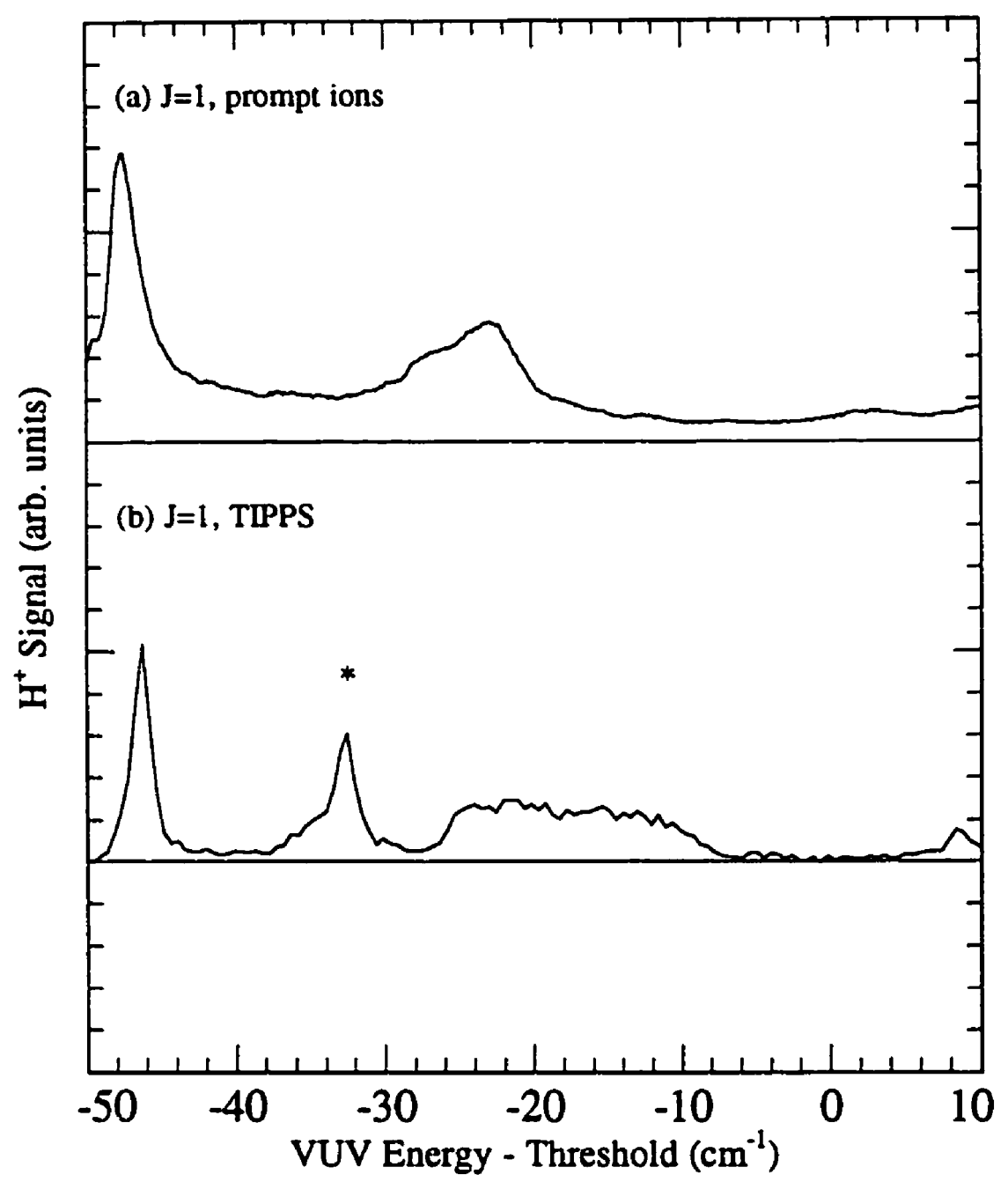

Figure 6.4: (a) $\mathrm{H}^{+}$prompt ion yield and (b) TIPPS spectrum of HF at the $J=1$ threshold. The energy scale is relative to the $J=1$ field-free ion-pair formation threshold. Conditions are identical to those of Fig. 6.3. 


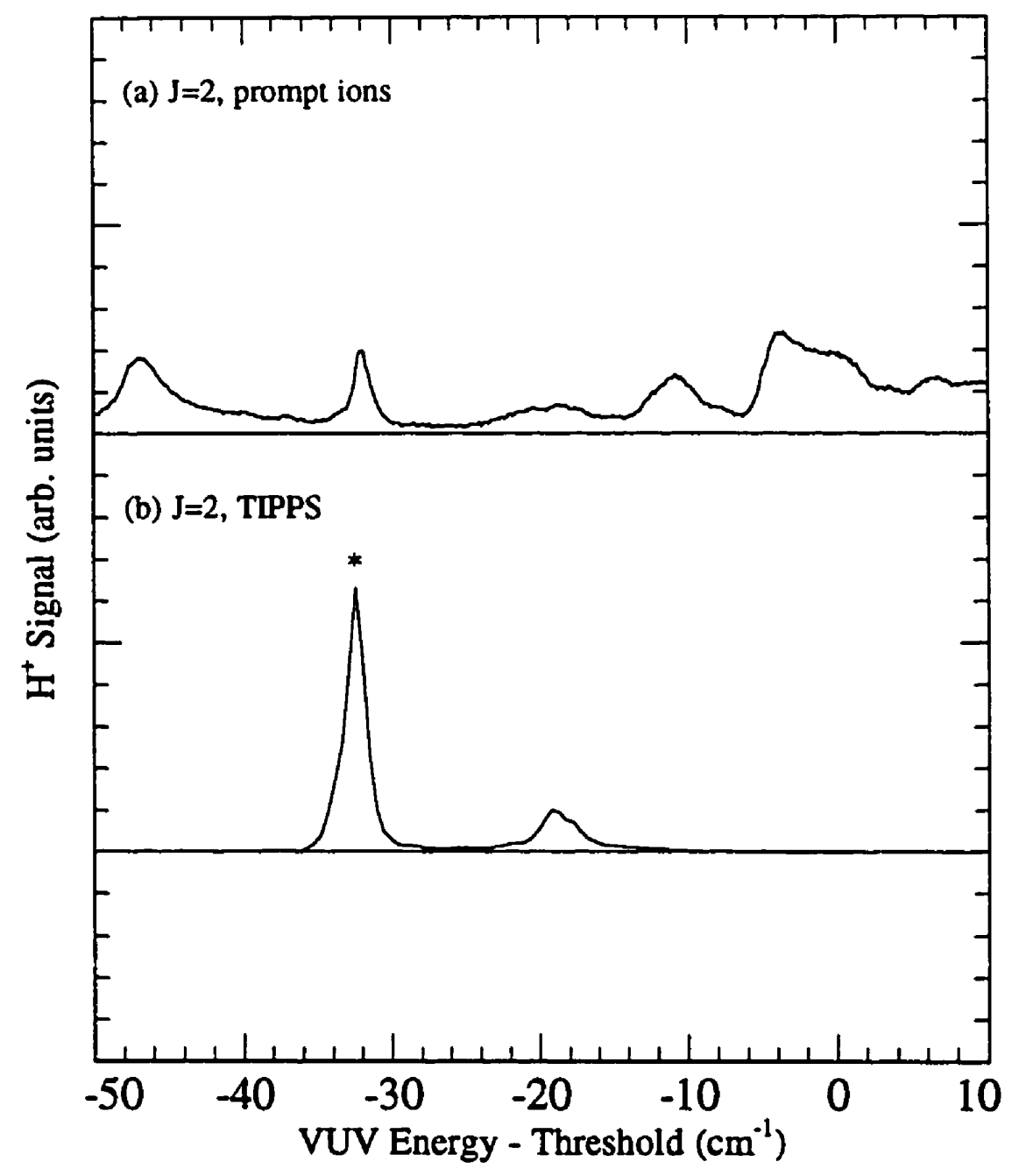

Figure 6.5: (a) $\mathrm{H}^{+}$prompt ion yield and (b) TIPPS spectrum of $\mathrm{HF}$ at the $J=2$ threshold. The energy scale is relative to the $J=2$ field-free ion-pair formation threshold. Conditions are identical to those of Fig. 6.3. 


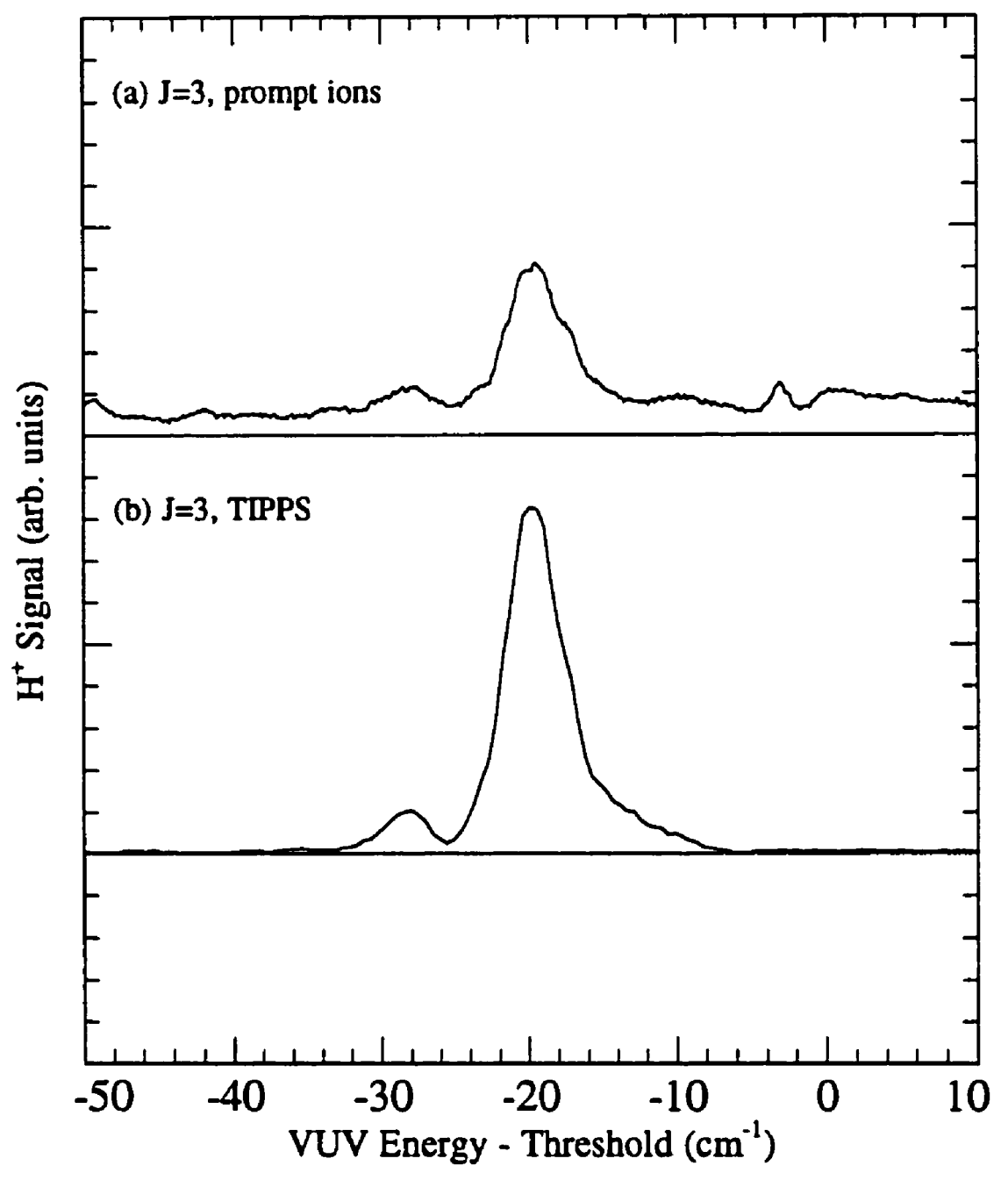

Figure 6.6: (a) $\mathrm{H}^{+}$prompt ion yield and (b) TIPPS spectrum of $\mathrm{HF}$ at the $J=3$ threshold. The energy scale is relative to the $J=3$ field-free ion-pair formation threshold. Conditions are identical to those of Fig. 6.3. 


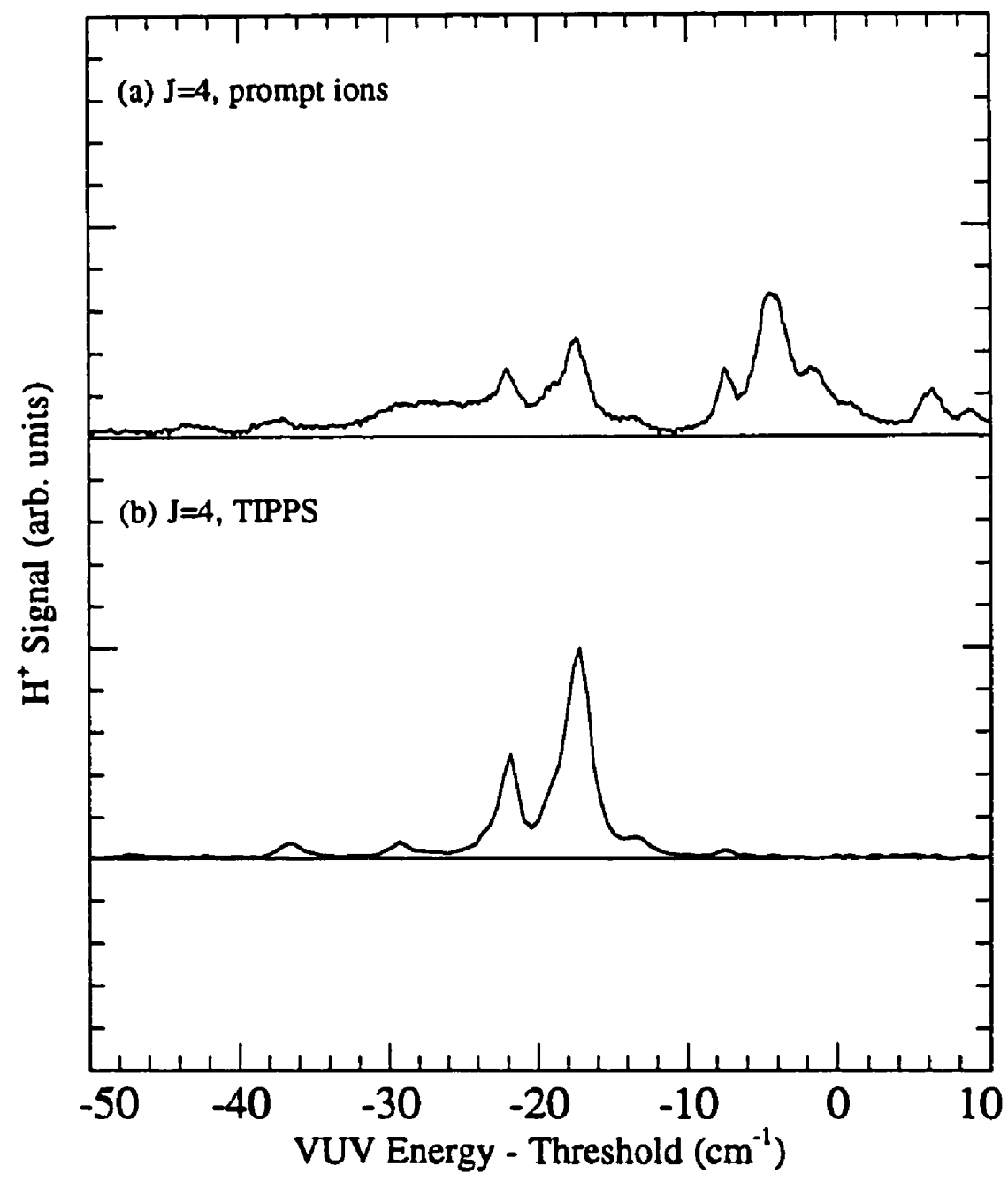

Figure 6.7: (a) $\mathrm{H}^{+}$prompt ion yield and (b) TIPPS spectrum of $\mathrm{HF}$ at the $J=4$ threshold. The energy scale is relative to the $J=4$ field-free ion-pair formation threshold. Conditions are identical to those of Fig. 6.3. 


\subsection{Determination of $D_{0}(\mathrm{HF})$}

\subsubsection{Extrapolation to Determine the Field-Free Ion-Pair Dissociation Threshold}

The different factors affecting MATI lineshapes were discussed in Section 3.3. The extrapolation procedure to determine ionization thresholds depends on the discrimination pulse survival probability dominating the shape of the blue edge of the line. In this case, Eqn. 3.5 is valid. However, if the probability of exciting Rydberg states is varying strongly in the vicinity of the threshold, or any other factors are influencing the shape of the blue-edge, Eqn. 3.5 will not be valid, hindering determination the field-free ionization threshold. The same considerations apply to TIPPS spectra. If the probability of producing ion-pairs varies strongly in the vicinity of the threshold, the shifting of $E_{1 / 2}$ with $F_{d}$ may not obey Eqn. 3.5. Therefore, based on the structure observed in Fig. 6.2 problems might be expected in applying the extrapolation procedure. Fortunately, both the $J=0$ and $J=1$ lines of the HF TIPPS spectrum have quite broad enhancements in the vicinity of their blue edges. Consequently, these blue edges shift sensibly with varying discrimination field (see Fig. 6.8). The plots of $E_{1 / 2}$ vs. $\sqrt{F_{d}}$ for both the $J=0$ and $J=1$ show data points falling close to straight lines, allowing determinations of $E_{\mathrm{H}^{+}, F^{-}}$by extrapolation (see Fig. 6.9). The field-free threshold is determined to be: $129558.2 \pm 0.3 \mathrm{~cm}^{-1}$ (where the errors due to photon energy calibration and extrapolation have been added in quadrature).

The dissociation energy of HF may now be determined through: ${ }^{2}$

$$
D_{0}(\mathrm{HF})=E_{\mathrm{H}+, \mathrm{F}^{-}}-I P(\mathrm{H})+E A(\mathrm{~F})
$$

where $I P(\mathrm{H})$ is the ionization potential of the hydrogen atom, and $E A(F)$ is the electron affinity of the fluorine atom. Blondel et al. [130] have determined $E A(F)=27432.440 \pm$

\footnotetext{
${ }^{2}$ This relationship has been used previously, by Chupka and Berkowitz et al. [31] to determine $D_{0}$ (HF) from the threshold for photoion-pair formation.
} 


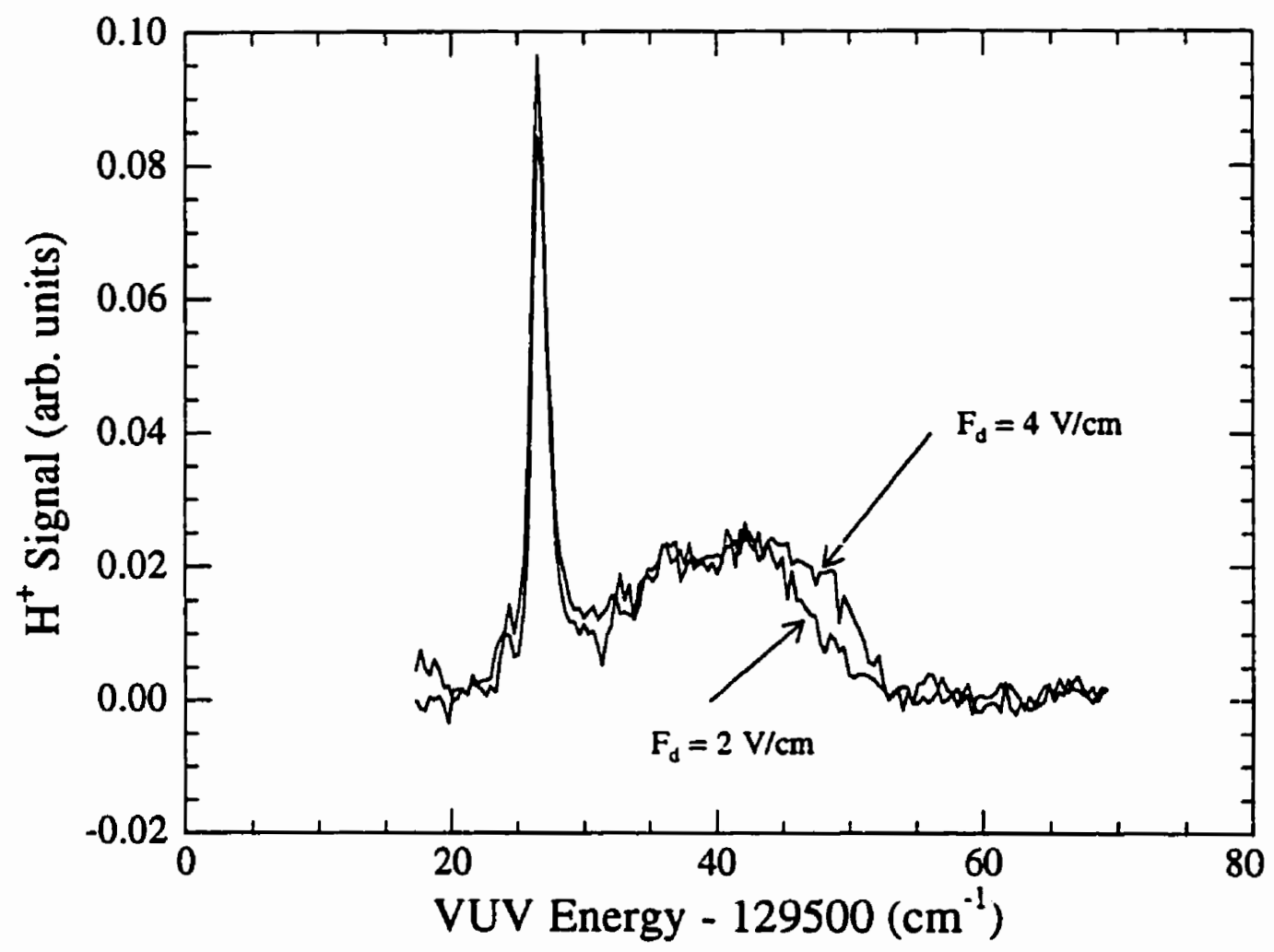

Figure 6.8: TIPPS spectra of HF for two different discrimination fields. For both spectra, the discrimination field was pulsed on $0.2 \mu$ s after photoexcitation, and an extraction field of $60 \mathrm{~V} / \mathrm{cm}$, was pulsed on $4.4 \mu \mathrm{s}$ after photoexcitation. 


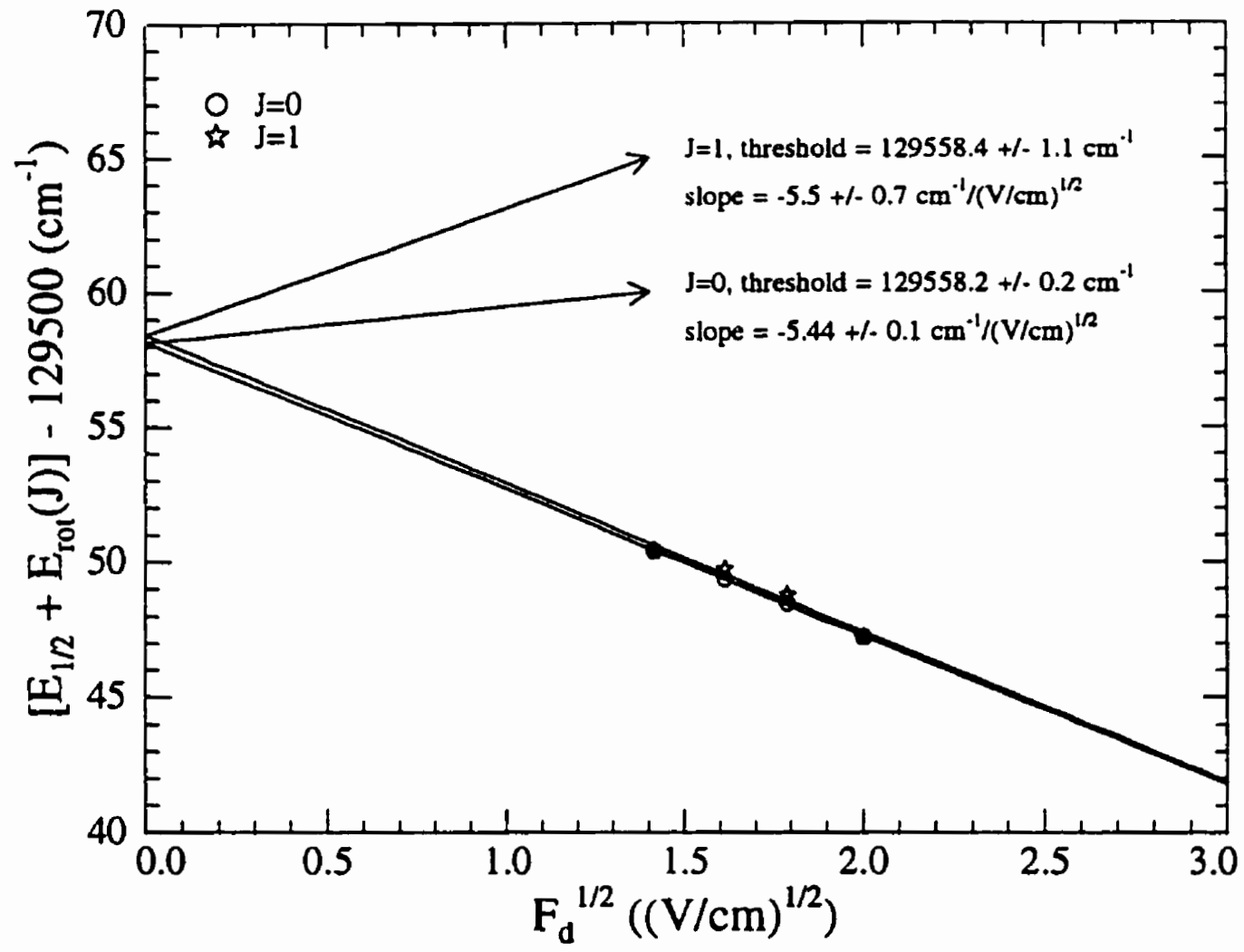

Figure 6.9: Extrapolation to determine the field-free ion-pair formation threshold for HF. The positions of the blue edges of a series of spectra taken at different discrimination fields are plotted as a function of the square root of the discrimination field. The $J=1$ data has been shifted up in energy by the $J=0, J=1$ energy level difference in the HF ground vibronic state $\left(E_{\text {rot }}(0)=0 \mathrm{~cm}^{-1}\right.$ and $\left.E_{\text {rot }}(1)=41.11 \mathrm{~cm}^{-1}\right)$. The extrapolated zero-field thresholds for the two $J$ levels are labeled. 
$0.025 \mathrm{~cm}^{-1}$. Substituting this value of $E A(\mathrm{~F})$ together with $I P(\mathrm{H})=109678.8 \mathrm{~cm}^{-1}$ (from Johnson and Soff [111]) into Eqn. 6.1, a value of $D_{0}(\mathrm{HF})=47311.8 \pm 0.3 \mathrm{~cm}^{-1}$ is obtained.

The determination of $E_{\mathrm{H}^{+}, \mathrm{F}^{-}}$dominates the uncertainty in $D_{0}(\mathrm{HF})$. This differs from the analogous determination of $D_{0}(\mathrm{HCl})$ presented in Chapter 4 , where the best literature value of $E A(\mathrm{Cl})$ has an uncertainty of $0.5 \pm \mathrm{cm}^{-1}$, which contributes to the uncertainty in $D_{0}(\mathrm{HCl})$.

\subsubsection{Current Literature Value for $D_{0}(\mathrm{HF})$}

The most recent determination of $D_{0}(\mathrm{HF})$ is that of Zemke et al. [126]. Their approach was to "match" low- $R$ values of the ground state potential derived from the inversion of spectroscopic data, with the expected high- $R$ asymptotic behavior by adjusting the dissociation asymptote to (1) allow the low- $R$ and high- $R$ potentials to merge smoothly, and (2) reproduce the energy of an experimentally observed level very close to the dissociation asymptote.

The long-range form of the potential chosen was:

$$
V_{L R}(R)=D_{c}-A \exp (-\alpha R)-\frac{C_{6}}{R^{6}}-\frac{C_{8}}{R^{8}}-\frac{C_{10}}{R^{10}}
$$

where $C_{6}, C_{8}, C_{10}, A$ and $\alpha$ were fixed by previous work, and $D_{e}$ - the depth of the potential well - was adjusted to satisfy one of the two criteria mentioned above. The dissociation energy $D_{0}$ may be computed by subtracting the zero point vibrational energy from $D_{e}{ }^{3}$

\footnotetext{
${ }^{3}$ From Herzberg [4], Section (III,2,a) and (III,2,c):

$$
\begin{array}{r}
D_{0} \approx D_{e}-( \\
\left(\frac{1}{2} \omega_{e}-\frac{1}{4} \omega_{e} x_{e}+\frac{1}{8} \omega_{e} y_{e}+\frac{B_{e}}{4}\right. \\
\left.+\frac{a_{e} \omega_{e}}{12 B_{e}}+\frac{a_{e}^{2} \omega_{e}^{2}}{144 B_{e}^{3}}-\frac{1}{4} \omega_{e} x_{e}\right) .
\end{array}
$$

For HF, Huber and Herzberg's compilation [107] lists $\omega_{e}=4138.32 \mathrm{~cm}^{-1}, \omega_{e} x_{e}=89.88 \mathrm{~cm}^{-1}, w_{e} y_{e}=$ $0.184 \mathrm{~cm}^{-1}, B_{\varepsilon}=20.9557 \mathrm{~cm}^{-1}, \alpha_{\varepsilon}=0.798 \mathrm{~cm}^{-1}$, and so $D_{0}(\mathrm{HF}) \approx D_{\mathrm{e}}(\mathrm{HF})-2051 \mathrm{~cm}^{-1}$. Zemke et
} 
The short-range potential for the ground state and non-adiabatic correction terms had previously been derived from experimental data [131]. By adjusting $D_{e}$, so that this potential smoothly merged with the long-range potential at low- $R$, Zemke et al. [126] found $D_{0}=47312 \pm 8 \mathrm{~cm}^{-1}$, where the error is based on the uncertainty in the parameters describing the long-range potential $\left(C_{6}, C_{8}, C_{10}, A, \alpha\right)$.

Abandoning the criteria of smoothness at low- $R$, the energy levels of the "merged" potential may be computed. For DiLonardo and Douglas's [1] spectroscopically determined level $v=19, J=9$, the energy is computed as a function of $D_{e}$. The experimental energy level is reproduced when

$$
D_{0}=47311 \pm 5 \mathrm{~cm}^{-1} \text {. }
$$

The uncertainties in the position of the experimental line, and the parameters describing the long-range potential $\left(C_{6}, C_{8}, C_{10}, A, \alpha\right)$ give the uncertainty of $\pm 5 \mathrm{~cm}^{-1}$. This value of $D_{0}(\mathrm{HF})$ agrees with DiLonardo and Douglas's [1] original estimate $D_{0}=47333 \pm 60$ $\mathrm{cm}^{-1}$ to within their uncertainty. This value was determined by the technique of "limiting curve of dissociation" discussed in Section (VII,2) of Herzberg [4].

In Section 4.5.3 it was discussed how the dissociation energy of $\mathrm{HCl}$ is determined from the standard enthalpy of formation of $\mathrm{HCl}, \Delta H_{j 0}^{\ominus}(\mathrm{HCl})$, the dissociation energy of $\mathrm{H}_{2}, \mathrm{D}_{0}\left(\mathrm{H}_{2}\right)$, and the dissociation energy of $\mathrm{Cl}_{2}, \mathrm{D}_{0}\left(\mathrm{Cl}_{2}\right)$. For the case of HF, using the JANAF [114] recommended values: ${ }^{4}$

$$
\begin{aligned}
D_{0}\left(\mathrm{H}_{2}\right) & =36118.3 \pm 1 \mathrm{~cm}^{-1} \\
D_{0}\left(\mathrm{~F}_{2}\right) & =12920 \pm 50 \mathrm{~cm}^{-1} \\
\Delta H_{\rho 0}^{\ominus}(\mathrm{HF}) & =22779 \pm 67 \mathrm{~cm}^{-1},
\end{aligned}
$$

al. [126] use an identical correction, which presumably they could derive by solving for the energy level $v=0, J=0$ in the potential.

${ }^{4}$ The original source of the $\Delta H_{j 0}^{\ominus}(\mathrm{HF})$ recommended in the JANAF tables was not accessible, so it is unclear whether or not this value is based on measurements of $D_{0}(\mathrm{HF}), D_{0}\left(\mathrm{H}_{2}\right)$ and $D_{0}\left(F_{2}\right)$ - rather than being measured by thermochemical means, like calorimetry, as in the case of $\mathrm{HCl}$. If the recommended $\Delta H_{\text {fo }}^{\ominus}(\mathrm{HF})$ is based on a value of $D_{0}(\mathrm{HF})$, the determination given here is invalid, since it is circular. 
the dissociation energy of HF is computed to be

$$
D_{0}(\mathrm{HF})=47298 \pm 84 \mathrm{~cm}^{-1}
$$

As shown in Section 4.5.3, the analogous determination for $\mathrm{HCl}$ is more precise $( \pm 18$ $\mathrm{cm}^{-1}$ ) since both $D_{0}\left(\mathrm{Cl}_{2}\right)$ and $\Delta H_{f 0}^{\ominus}(\mathrm{HCl})$, are better known than $D_{0}\left(\mathrm{~F}_{2}\right)$ and $\Delta H_{f 0}^{\ominus}(\mathrm{HF})$.

The present determination of $D_{0}(\mathrm{HF})=47311.8 \pm 0.3 \mathrm{~cm}^{-1}$ is within the error bars of the best current literature value of Zemke et al. [126] $\left(D_{0}(\mathrm{HF})=47311 \pm 8 \mathrm{~cm}^{-1}\right)$. 


\section{Chapter 7}

\section{Concluding Remarks}

This thesis has introduced a technique for observing the electric-field induced dissociation of high- $v$ ion-pair states: Threshold Ion-Pair Production Spectroscopy (TIPPS). Using this method, it is possible to determine the energetic thresholds for splitting a molecule into ion-pairs. For the $\mathrm{HCl}$ molecule, this has significantly improved the literature value for the neutral dissociation energy $D_{0}(\mathrm{HCl})$. Since nothing appears to restrict application of the TIPPS method to diatomics, the determination of bond-energies in polyatomics many of which are known only thermochemically - is a compelling future application of this technique.

Since $\mathrm{HF}$ and $\mathrm{HCl}$ have such similar electronic structures, one might expect that their TIPPS spectra would be similar. This is not the case, as shown in a comparison of Fig. 4.2b) with Fig. 6.2a). The HF TIPPS spectra are very structured, with sharp resonances within each individual threshold peak, whilst the $\mathrm{HCl}$ spectra show little signature of individual resonances. Analysis of these resonances - which is still at a preliminary stage - should help in understanding the mechanism for the production of ion-pairs.

The partners of an ion-pair may not necessarily be in their ground states. This is demonstrated in the TIPPS spectrum of $\mathrm{O}_{2}$ presented in Chapter 5. A set of peaks have been positively identified as corresponding to the electric field induced dissociation of 
high-v ion-pair states in which the $\mathrm{O}^{-}$was in the second, excited spin-orbit state. If the spin-orbit splitting in $\mathrm{O}^{-}$were not known, this spectrum could provide the value. This suggests that the TIPPS technique could be applied to negative ion-spectroscopy. Another possible application, discussed in Chapter 5 , is the study of the reaction of state-selected negative ions. In particular, the technique described in this thesis could be modified to produce a beam of $\mathrm{O}^{-}$ions in either spin-orbit state, for reaction studies. No methods for state-selected production of negative ions have been previously demonstrated.

To gain a more complete understanding of high- $v$ ion-pair states, and their similarities in behaviour to Rydberg states, it would be desirable to be able to selectively excite specific $v$, rather than an unresolved range as done in this work. Individual ion-pair states have been studied at low- $v$, but unfortunately the Rydberg-like behaviour is not easily observed, until the binding energy is relatively weak, at high-v. For example, it would be impractical to generate the required electric fields to dissociate any of the ion-pair states of the $B$ state observed in DiLonardo and Douglas's work, which observed levels as high as $v=73$ [1]. To excite individual $v$-states near the threshold, it is desirable to have a high-resolution excitation source. For a given binding energy, Rydberg state spacing is much less dense than that of high-v ion-pair states. Calculations show that excitation of individual high- $v$ ion-pair states of $\mathrm{H}_{2}$ is practical, if they are excited with a standard etalon equipped pulsed dye laser. For this, it would be necessary to use a two-colour double resonance scheme, like Pratt and co-workers [14]. Experiments such as this are being planned by several groups.

From the preceeding paragraphs, it is apparent that there are several fruitful lines of research concerning the electric-field induced dissociation of high-v ion-pair states. However, the author feels sorry for future researchers in this field, as the analogy with Rydberg states has invited an unfortunate nickname for molecules in high-v ion-pair states. Workers will be forced to admit that their research is the subject of "Rydicules". 


\section{Appendix A}

\section{Wavelength Calibration Procedure}

\section{A.1 Introduction}

As with previous work in the Extreme Ultraviolet Lab [61, 128, 132], dye laser wavelength calibration was based on the observation of tabulated atomic lines using the optogalvanic effect in a hollow-cathode discharge [133]. However, because of the desirability in obtaining an accurate VUV energy calibration for dissociation threshold determination, several modifications to the procedure were made:

1. Transitions in $U$ instead of Ne were used,

2. Calibrations were performed shortly after data collection, and

3. The non-linearity observed in the grating drive mechanism was corrected by using etalon frequency markers.

These items will be discussed in this appendix. 
Optogalvanic spectroscopy is based on the principle that laser light can influence the properties of a gaseous electrical discharge (the optogalvanic effect), and that these effects may be measured electrically in an external sırcuit. By inducing transitions in either the fill gas (e.g. Ne) or sputtered species (e.g. U), one can enhance or reduce the number of charge carriers, thereby changing the $V-I$ curve of the discharge. The light from the pulsed dye laser induces a transient every $100 \mathrm{~ms}$ (the laser repetition rate) in the small external circuit pictured in Fig. A.1. This signal is gated using a boxcar integrator and the output of the boxcar is digitized and recorded as a function of excitation wavelength. The resultant spectrum has peaks which can be assigned to atomic transitions which have been tabulated by other authors using independent methods.

The hollow cathode lamp used was a commercial product (Westinghouse WL 22826 or SCP Science 030-150-922) intended for use in Atomic Absorption (AA) spectroscopy. The cathode material was $U$, and as discussed in the following section, transitions in either the fill gas ( 5 torr of $\mathrm{Ne}$ ), or sputtered species (U) could be observed, depending on the conditions.

\section{A.2 Observation of Uranium Optogalvanic Transitions}

A previous worker [134] had constructed a circuit similar to that in Fig. A.1. However the ballast resistor $(R b)$ was selectable between three values: $R b=46,153$ or $305 \mathrm{k} \Omega$. The voltage drop across the lamp is roughly $200 \mathrm{~V}$ [135], so when using the power supply available in the lab $(400 \mathrm{~V})$, with the lowest ballast resistance $(46 \mathrm{k} \Omega)$, the lamp current was $4 \mathrm{~mA}$. With this current, it was not possible to observe any $U$ transitions. However, transitions in the Ne fill gas were observed and some of these could be assigned. These transitions had been used for calibration by both the author [21] and previous workers in the lab $[61,128,132]$. Unfortunately, the observed Ne line density is low (there are only 5 assignable lines between $560 \mathrm{~nm}$ and $570 \mathrm{~nm}$ ), and the accuracies of the line positions in the standard compilations $[136,137]$ are difficult to ascertain. To investigate the observed 


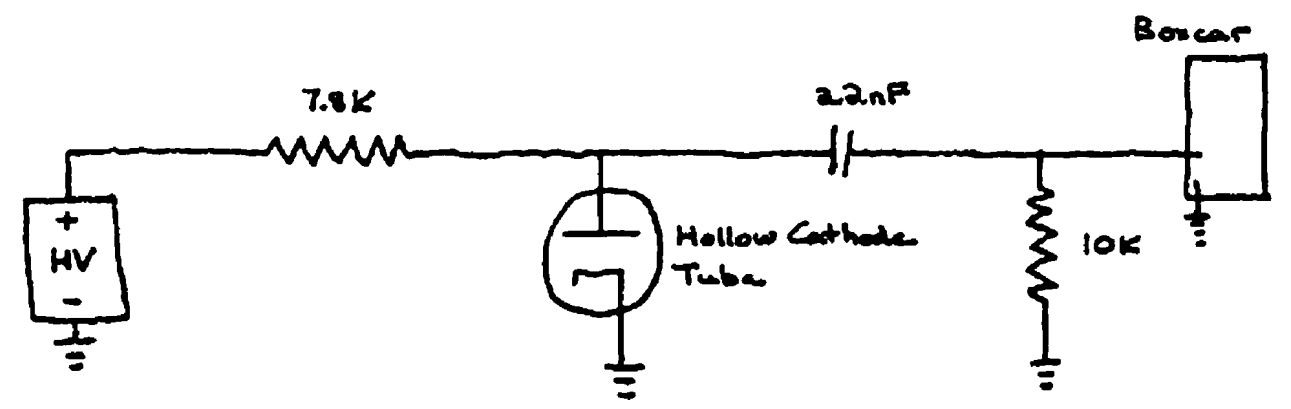

Figure A.1: Circuit for observation of optogalvanic effect. Modeled after Ref. [133]. The $7.8 \mathrm{k} \Omega$ resistor, should be a high wattage type (in this case a $3 \mathrm{~W}, 15 \mathrm{k} \Omega$ resistor and a $3 \mathrm{~W}, 16 \mathrm{k} \Omega$ resistor were connected in parallel). The capacitor should be able to hold off 200 VDC (a ceramic disc type is suitable). 
grating drive non-linearity and have more confidence in the calibration, it was desired to obtain the $U$ transitions used by other workers [133]. ${ }^{1}$

Since sputtered atom density is proportional to the square of the discharge current [133], the low ballast resistance of $7.8 \mathrm{k} \Omega$ was used. This allowed the lamp to be operated at approximately $25 \mathrm{~mA}$ (the manufacturer's recommended maximum) with the $400 \mathrm{~V}$ power supply available. Operating the lamp at these higher currents permitted observation of the transitions due to sputtered $U$. Interestingly, some of the $\mathrm{Ne}$ transitions, which were observed at lower currents, disappeared. Over the limited wavelength range studied, no new Ne lines appeared at the higher current. Therefore if the Ne line density and tabulated accuracy are acceptable, it may be desirable to operate the lamp at the greatly reduced currents, which would probably lengthen its life.

Figure A.2 shows a typical optogalvanic spectrum taken with high current. The lines may be assigned to transitions in $U$ using the Los Alamos Scientific Laboratory (LASL) line list [139]. The transitions in this compilation were observed in emission from a similar hollow-cathode lamp (operated at higher currents) by a Fourier Transform Spectrometer (FTS). Several of the tabulated lines in the LASL line list have been checked using a highly accurate lambda meter located at Joint Laboratory for Astrophysics (JLLA) [140]. Agreement was excellent $\left( \pm 0.002 \mathrm{~cm}^{-1}\right)$, and so the accuracy of the LASL compilation appears more than sufficient for this work.

As noted by Dovichi et al. [133], there is a strong correlation between the relative emission intensities tabulated in Ref. [139] and the observed optogalvanic signal strengths from line to line. This helps assign the observed spectrum. ${ }^{2}$

\footnotetext{
${ }^{1}$ Observation of laser induced fluorescence from $I_{2}$ can often provide a suitable calibration for dye lasers. However, this is over a limited range of the spectrum [138], and the line density is so high that individual transitions are not easily resolved with pulsed dye lasers.

${ }^{2}$ It is observed that lines which originate from $U$ atoms in their ground state are stronger than one would expect based on the emission intensities in the LASL line list (compared to lines originating from excited U atoms). This suggests that the discharge is not as hot as for the LASL observations.
} 


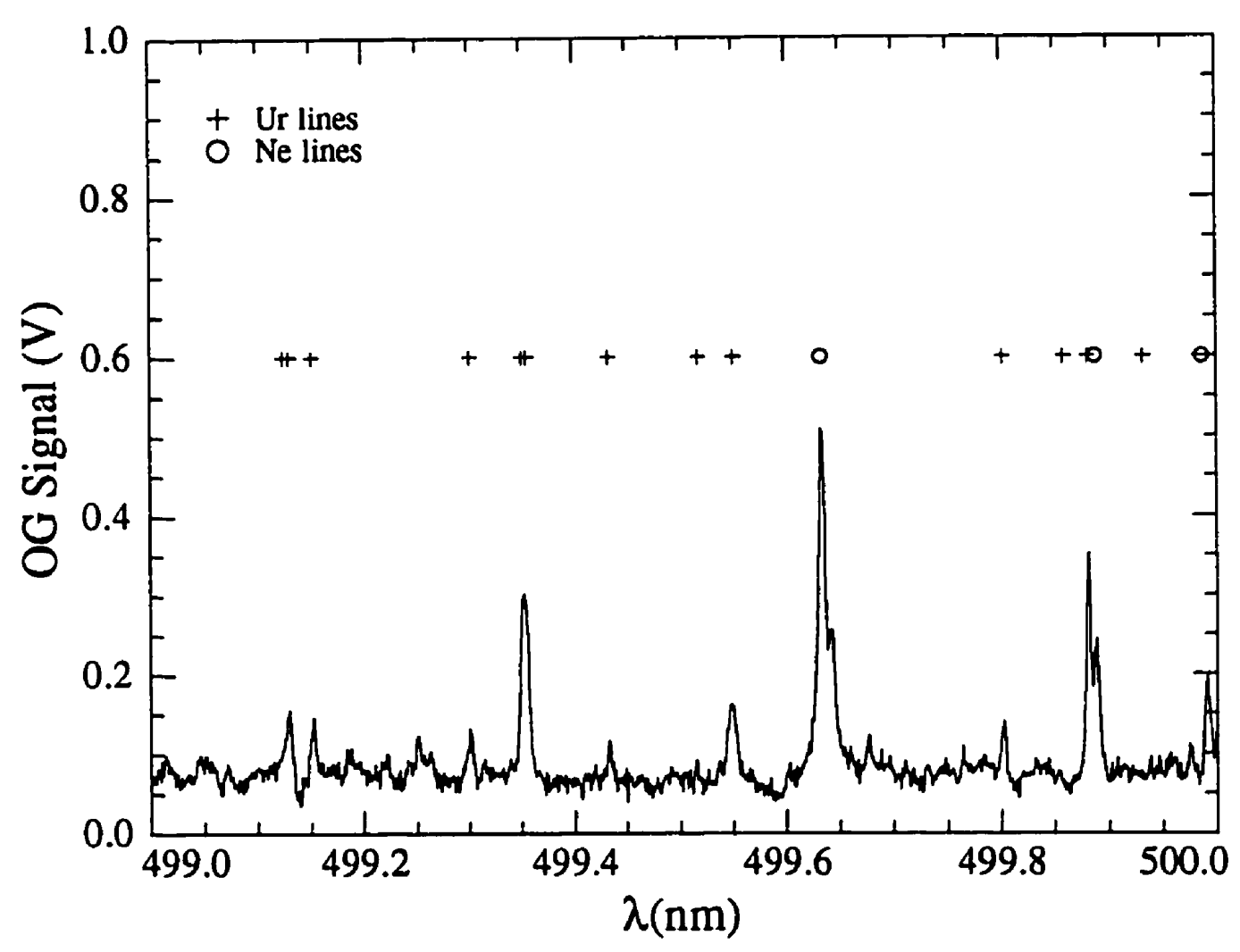

Figure A.2: Sample U optogalvanic spectrum. Vertical scale is the average voltage recorded by the boxcar over a $3.4 \mu$ s time-period starting $0.3 \mu$ s after the light pulse. Fluence directed into lamp was $\approx 10 \mu \mathrm{J} /$ pulse, focused from a collimated beam width of $1 \mathrm{~mm}$ using a $25 \mathrm{~cm}$ focal length lens. 
Since the $U$ atom is heavy, Doppler broadening of the transitions is expected to be less than the laser linewidth. At a temperature of $4660 \mathrm{~K}$ (as found in Ref. [139]), the Doppler linewidths of visible wavelength transitions in $U$ are roughly $0.1 \mathrm{~cm}^{-1}$. Likewise, pressure broadening is not expected to be large at the tube pressures. Despite this, linewidths of the observed $U$ transitions exceeded the laser linewidth. Some lines were broader than others, and it was found that closely spaced lines could be more easily resolved when the laser power directed into the lamp was lower - at the expense of reduced signal. Thus, it seems most likely that saturation (power) broadening is occurring (see, for example, Ref. [82]). Since numerous lines were unresolved, it is desirable to reduce this broadening to increase the number of assignments, and thus quality of the calibration. Since the signal to noise ratio of the optogalvanic signal seemed to improve with larger laser powers, further efforts should concentrate on either reducing the noise, or increasing the sputtered $U$ density - both of which would enable lower laser powers to produce a more detectable optogalvanic signal. Higher currents (above the lamp's rated maximum) could be used to achieve higher $U$ densities (see Ref. [139]), but some form of active cooling would be required and the anticipated reduction in lamp lifetime would have to be investigated.

\section{A.3 Effects of Air Temperature and Pressure on the Cali- bration}

As discussed in Section 2.2.4, a particular orientation of the grating with respect to the dye laser cavity axis, results in lasing at a certain air wavelength. The frequency of the light (or corresponding vacuum wavelength) then depends on the refractive index of the air surrounding the grating. The refractive index $n$ of air is a function of its temperature, pressure, and the specific frequency involved. Cole [141] summarizes the relevant formulae:

$$
(n-1)=\left(n_{s}-1\right) \frac{1+\alpha t_{s}}{1+\alpha t}\left(\frac{P}{P_{s}}\right)
$$


where $t_{s}=15^{\circ} \mathrm{C}, t$ is the air temperature in ${ }^{\circ} \mathrm{C}, P$ is the pressure in torr of the surrounding air, $P_{s}=760$ torr, and $\alpha=0.00366$. The variation of the refractive index of "standard air", $n_{s}$, is given by [141]:

$$
\left(n_{s}-1\right) 10^{8}=6432.8+\frac{2949810}{146-\nu^{2}}+\frac{25540}{41-\nu^{2}}
$$

where $\nu$ is the vacuum wavenumber in $\mu \mathrm{m}^{-1}$.

Assuming grating positioning is reproducible from day to day, the relationship between grating position and dye laser frequency will vary because of fluctuations in temperature and pressure, since

$$
\lambda_{v a c}=n \lambda_{\text {air }}
$$

What are the typical fluctuations in temperature and pressure from day to day?

Figure A.3 histograms the pressures recorded in the laboratory. The standard deviation of the distribution is 6.3 torr.

The temperature in the laboratory slowly rose by approximately $4{ }^{\circ} \mathrm{C}$ in the 2 hours following turning on the equipment required for the experiment. The temperature remained relatively constant $\left( \pm 0.5^{\circ} \mathrm{C}\right)$ for the rest of the day. Initial temperatures in the lab varied by about $1.5^{\circ} \mathrm{C}$. Thus provided the experiment and calibration are done after the initial heating period, the standard error in $t$ is roughly $2^{\circ} \mathrm{C}$.

Given these fluctuations in temperature and pressure from day to day, the variation in the final VUV energies can be estimated (assuming the air wavelengths stay constant). If the wavelength is roughly the same $\left(\lambda_{\text {air }} \approx 500 \mathrm{~nm}\right)$ for both dye lasers, and they are both doubled for four-wave mixing (the typical scenario), the final VUV energy $E_{V U V}$ is:

$$
E_{\mathrm{VuV}}\left(\text { in } \mathrm{cm}^{-1}\right) \approx \frac{6 \times 10^{7}}{n \lambda_{\text {air }}(\text { in } \mathrm{nm})}
$$

Using the standard formula for error propagation [142] (assuming $t$ and $P$ are uncorrelated):

$$
\sigma_{E_{\mathrm{VUV}}}=\sqrt{\left(\frac{\partial E_{\mathrm{VUV}}}{\partial t} \sigma_{t}\right)^{2}+\left(\frac{\partial E_{\mathrm{VUV}}}{\partial P} \sigma_{P}\right)^{2}}
$$




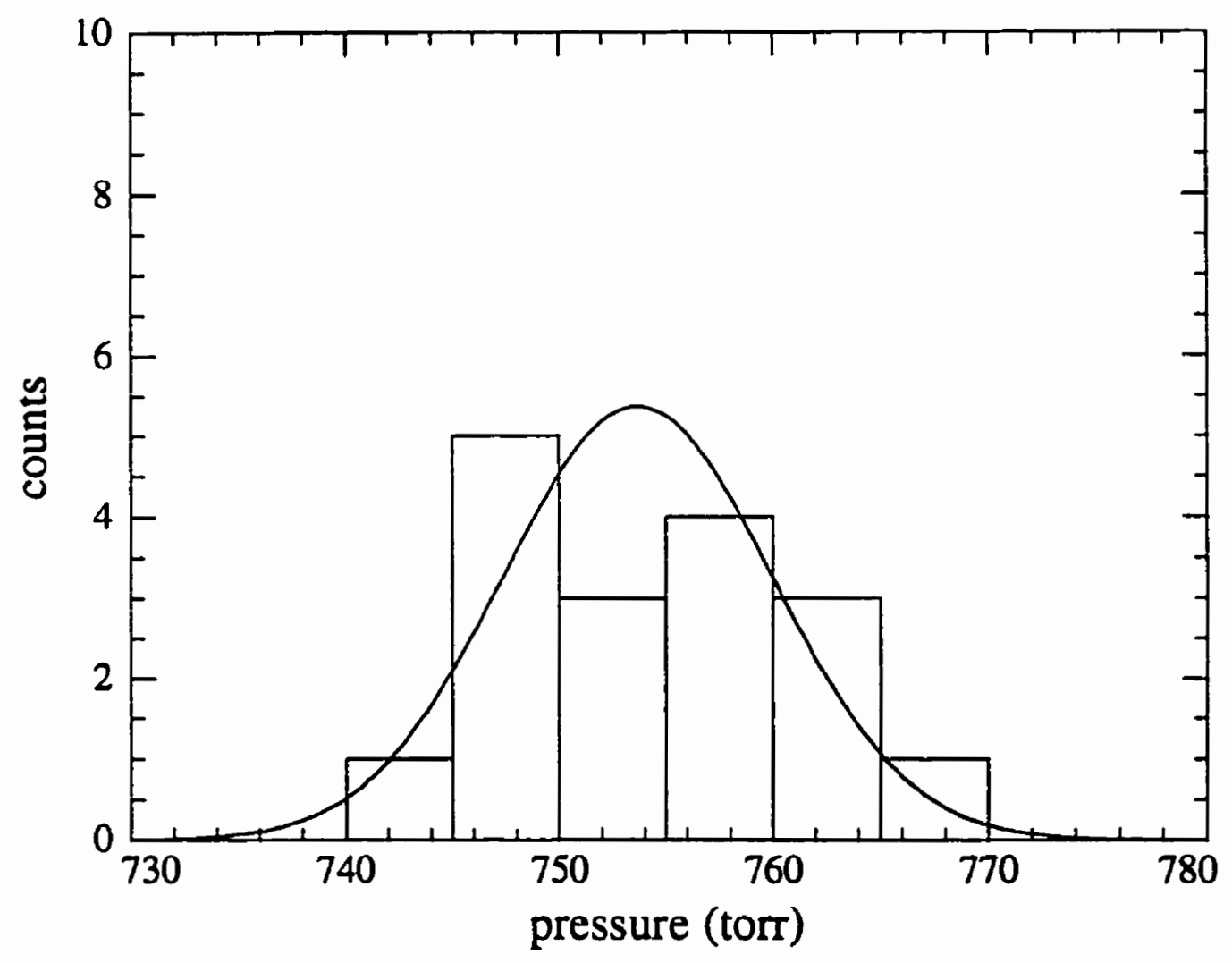

Figure A.3: Distribution of daily pressures in room C2-066. Seventeen individual daily pressure measurements are histogramed, and a normal distribution with the same mean and standard deviation is superimposed. The pressures were measured using a capacitance manometer (MKS 116A Baratron $\mu$ BAR). 
with $t=26 \pm 2{ }^{\circ} \mathrm{C}$ and $P=753.6 \pm 6.3$ torr, the uncertainty in the final energy is: $\sigma_{E_{\mathrm{VUV}}}=0.34 \mathrm{~cm}^{-1}$. However by monitoring both pressure and temperature shifts while the experiment and calibration was performed, it was ensured that pressure shifts were less than 2 torr and temperature shifts were less than $0.5^{\circ} \mathrm{C}$. Therefore the standard deviation of the final VUV energy due to temperature and pressure shifts within the day is $0.1 \mathrm{~cm}^{-1}$.

Initially it was hoped that by measuring $t$ and $P$, it would be possible to use a single calibration (relating stepper motor counter number to air wavelength) from day to day. Unfortunately this does not appear possible. There is some grating positioning irreproducibility which does not correlate with shifts in temperature or pressure. Consequently, it is imperative to calibrate the dye lasers each day. Fortunately, if the dye laser controller was left on, grating positioning appeared to be reproducible within the day. In fact, this was necessary, as the dye lasers were not calibrated during the scans, but rather, after.

This raises the general point of metrology problems associated with resonantly enhanced four wave mixing. Frequency calibration using the optogalvanic effect requires the wavelength to be scanned. This means that calibration of the $\omega_{\text {res }}$ dye laser must be done before or after a data collection scan (since $\omega_{\text {res }}$ remains fixed during the scan). This calibration will move the frequency off-resonance, requiring it to be moved back. This is convenient if the grating positioning is reproducible, and quite difficult if it is not. Obviously the requirement of scanability for calibration is not a problem for the $\omega_{\text {tun }}$ laser, which must be scanned anyways. It would be useful to have a calibration technique which did not require the $\omega_{r e s}$ laser to be scanned. A Fizeau wavemeter [143] would be suitable for this purpose. The resonance frequency could then be monitored in near real-time, and the optogalvanic and etalon equipment could be solely devoted to calibration of the $\omega_{\text {tun }}$ laser.

It is noted that in extremely high precision work done in the VUV and XUV $[144,86]$ the final frequency is derived from the harmonics of a single tunable laser, which is then 
scanned across the resonance being observed. This eases the metrology problem, since now one need only determine the frequency of one laser - which is being scanned. ${ }^{3}$ The introduction of two independent frequencies in resonantly enhanced four-wave sum mixing doubles the required effort. It seems as though some form of active stabilization of the resonance frequency laser to an atomic or molecular line would be best. The metrology problems of very high-resolution resonantly enhanced four-wave sum-frequency mixing [145] await further study.

\section{A.4 Scan Linearization}

The dye laser grating positioning is performed by a stepper motor. The manufacturer gives an approximate relationship relating stepper motor position $i$ to wavelength:

$$
\lambda_{\text {counter }}(\mathrm{nm})=\left[i \times 3.15 \times 10^{-3} \mathrm{~nm}+\lambda_{0}\right] / n
$$

where $i$ is an integer ranging from 0 to 285715, $n$ is the grating order (determined by the gain-bandwidth of the dye), and $\lambda_{0}$ is a constant specific to each dye laser. For convenience, calibrations will be discussed in terms of relating $\lambda_{\text {counter }}-$ the estimated wavelength - to the true vacuum wavelength $\lambda_{\text {vac }}$.

The assigned optogalvanic lines may be used to establish the relationship between the $\lambda_{\text {counter }}$ and the $\lambda_{\text {vac }}$. This relationship may then be used for any wavelengths in the range encompassed by the calibration points. Fig. A.4 shows the deviations between the counter wavelength at which a $U$ transition was observed, and the actual vacuum wavelength of the transition (from the LASL line list). With the high density of $U$ lines it is obvious that the deviation cannot be represented by a straight-line fit, whereas with the low density of Ne transitions it is difficult to make this conclusion (see pg. 59-61

\footnotetext{
${ }^{3}$ Very high-resolution work with pulsed sources requires the consideration of optical phase perturbations which occur in each step of frequency-mixing. One can no longer assume that the harmonic frequencies are integer multiples of the fundamental (see Ref.'s [85] and [86]).
} 
of [21]). The oscillatory-like deviation must be taken into account to achieve the best possible calibrations (the deviation from straight-line behavior is greater than the laser linewidth). Calibrations which did not take into account this deviation had unacceptably large errors in the final VUV energy. For example, it was not uncommon to observe deviations in linearity of $0.005 \mathrm{~nm}$ in the visible wavelength of both of the dye lasers. If these are not corrected, they can introduce errors in the VUV energy of up to 1.4 $\mathrm{cm}^{-1} .^{4}$ Before implementing a correction for the non-linearity, discrepancies of this size were noted between tabulated VUV energies and observed values. The solution was to "linearize" the wavelength scale by collecting etalon fringe patterns in addition to optogalvanic spectra.

While collecting the optogalvanic spectra, a small amount of light was split off and directed towards a diffuser, followed by a solid etalon $\left(\approx 0.67 \mathrm{~cm}^{-1}\right.$ Free Spectral Range, Lambda Physik, FL82), and a lens ( $1 \mathrm{~m}$ focal length). This projected a fringe pattern onto a screen approximately $1 \mathrm{~m}$ away. A photodiode was placed at the screen, and recorded the passing fringes as the wavelength was scanned.

Figure A.5 shows a fringe pattern and corresponding optogalvanic spectrum recorded simultaneously. The etalon marks off constant frequency intervals, thus there is a relationship between fringe number $(j)$ and frequency $(f)$ :

$$
f=j m+b
$$

where $m$ and $b$ are constants specific to the etalon and wavelength range.

Once a table of $\lambda_{\text {counter }}$ positions versus fringe number $j$ has been accumulated, interpolation may be used to determine the "fractional" fringe number of any $\lambda_{\text {counter }}$ position. 5 This fringe number may then be substituted into Eqn. A.6 to determine the true frequency, given $m$ and $b$.

\footnotetext{
The errors due to the non-linearity are not normally distributed. When varying $\omega_{\text {counser }}$ through a significantly large range, the non-linearity will be encountered, but the error is bounded. The $\omega_{r e s}$ dye laser may or may not be located in a "bad" region.

${ }^{5}$ Since there are approximately 60 fringes/nm, and typical scans are over several nanometers, it is
} 


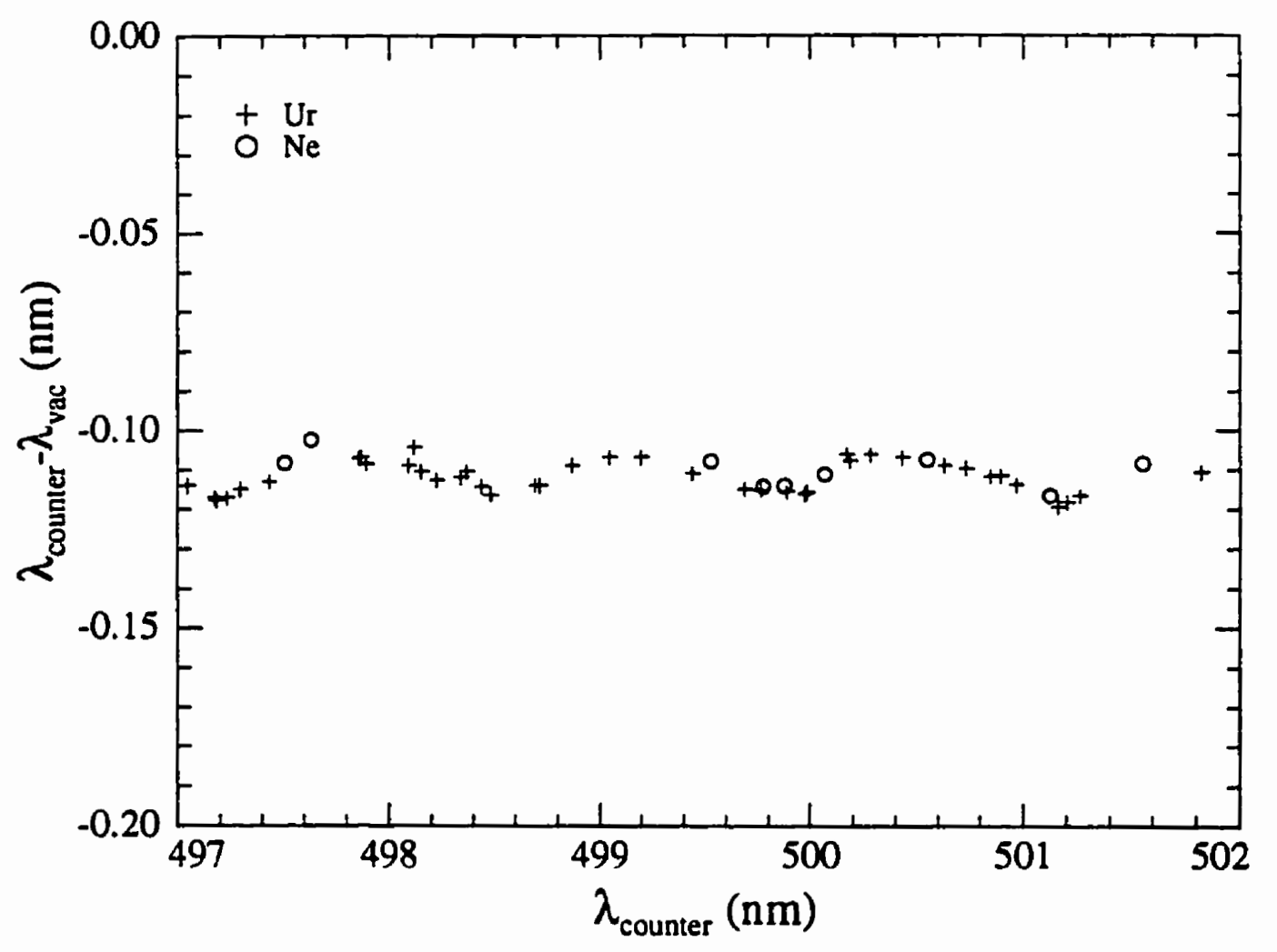

Figure A.4: Sample wavelength calibration using $U$ and Ne optogalvanic transitions. 


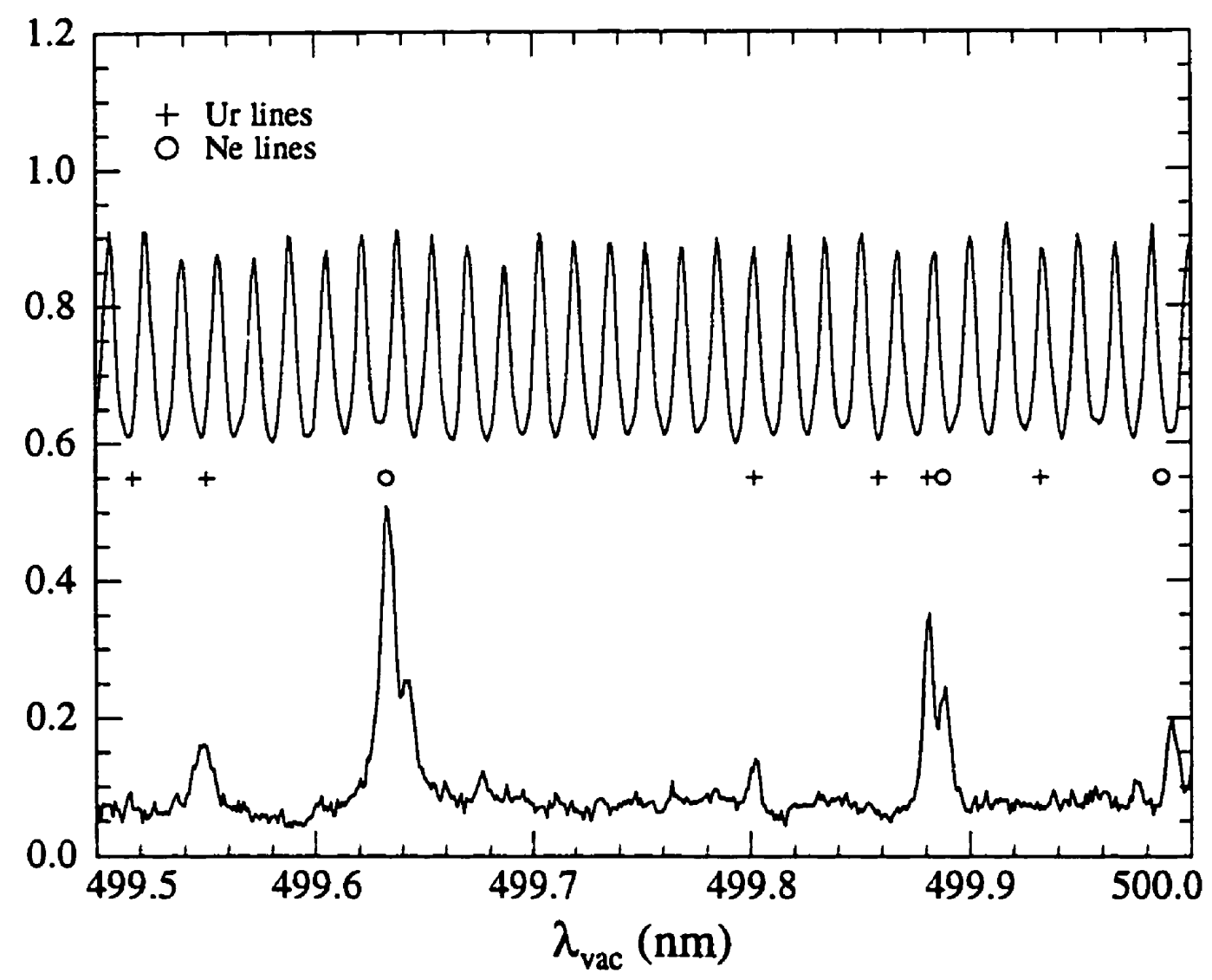

Figure A.5: Sample etalon pattern and U optogalvanic spectrum. 
The optogalvanic spectra are used to determine $m$ and $b$. The true frequencies of each $U$ line are known from the LASL compilation [139], and the $\lambda_{\text {counter }}$ positions of these transitions are observed in the optogalvanic spectrum. The $\lambda_{\text {counter }}$ positions can then be converted into fractional fringe numbers as discussed above. Thus, for each $U$ line, there is a frequency and fractional fringe number which must satisfy Eqn. A.6. The values of $m$ and $b$ may then be determined by least squares fitting all of the $U$ lines to this equation. Arbitrariness in absolute fringe labeling is absorbed by the constant $b$. (Fringe counting may start at any number, since this will merely change the value of $b$ found.)

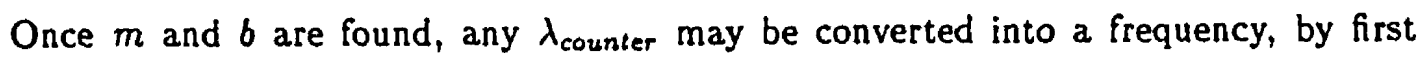
finding the fractional fringe number by interpolation, then converting this to a frequency using Eqn. A.6.

To illustrate this procedure, Fig. A.6 shows $\lambda_{\text {counter }}-\lambda_{\text {vac }}$ vs. $\lambda_{\text {counter }}$, where the $\lambda_{\text {vac }}$ values were derived from the calibration procedure discussed above. Overlaid are the observed $U$ positions (where the $\lambda_{\text {counter }}$ values are observed in the optogalvanic spectra, and the $\lambda_{\text {vac }}$ values are from the LASL compilation [139]). As seen, use of the etalon fringes helps correct the non-linearity, and subsequently reduce the error in the calibration.

The error in the calibration may be estimated by computing the standard deviation of the residual between the calibrated positions of the $U$ optogalvanic peaks, and their tabulated positions. This is typically less than $0.03 \mathrm{~cm}^{-1}$. Assuming the errors in the calibrations of the two lasers are uncorrelated, this gives a standard deviation in the final VUV energy of approximately $0.13 \mathrm{~cm}^{-1}$. When this error is combined with that estimated in Section A.3, due to fluctuations of laboratory temperature and pressure, the total error is $0.17 \mathrm{~cm}^{-1}$. This is a significant improvement over the errors of $1.4 \mathrm{~cm}^{-1}$ which were observed when the scan non-linearities were not taken into account.

impractical to accumulate this table by hand. Thus, an automatic fringe peak fitting program was written by the author. 


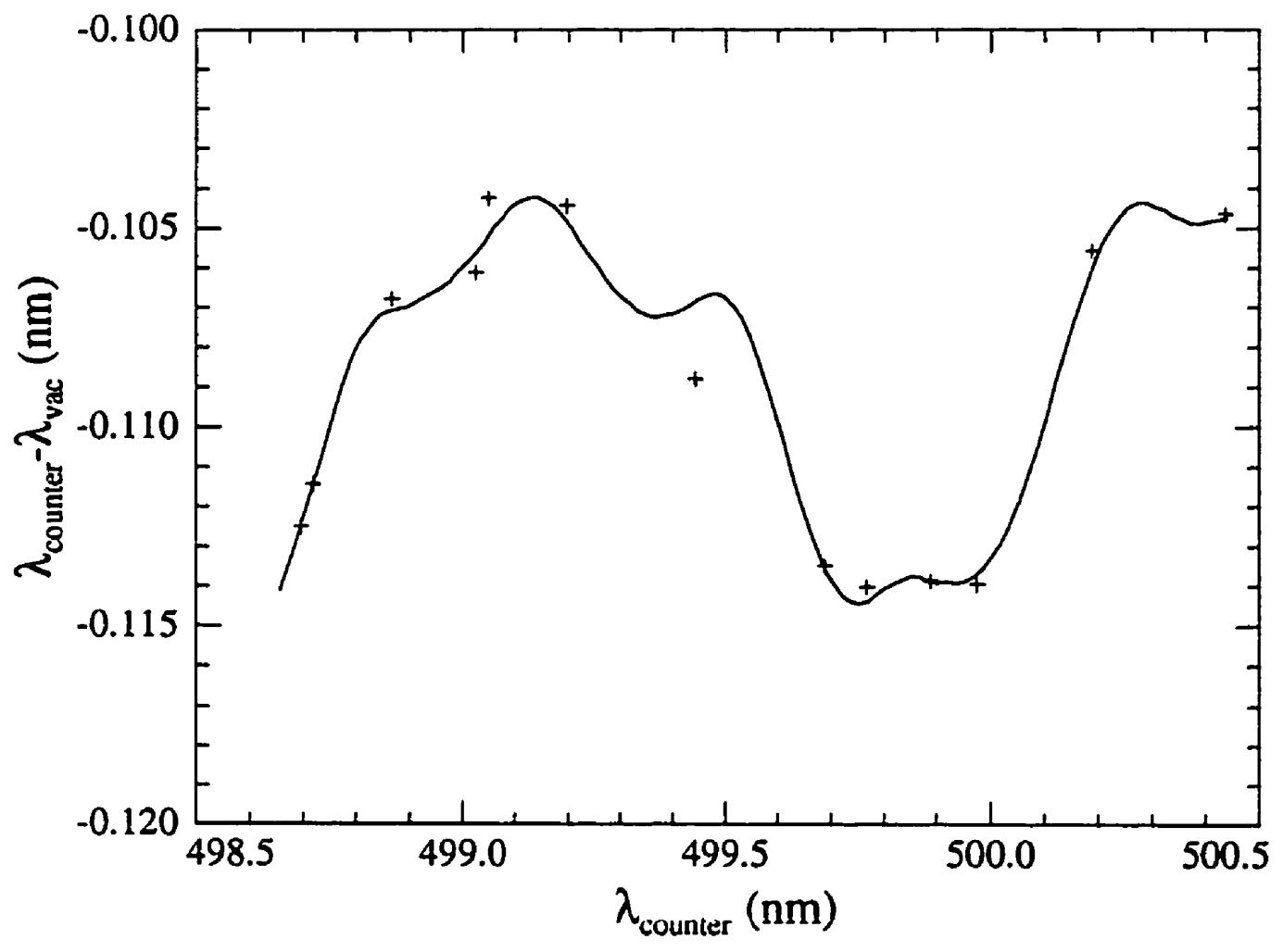

Figure A.6: Sample calibration using etalon fringes. The solid line shows the calibration which may be used to convert $\lambda_{\text {counter }}$ into $\lambda_{\text {vac }}$. The points show how the observed $U$ optogalvanic lines match this relationship. 


\section{Appendix B}

\section{The Efficiency of High- $n$}

Rydberg-State Stabilization in

Pulsed-Field Ionization

Zero-Kinetic-Energy

\section{Photoelectron Spectroscopy}

\section{B.1 Introduction}

As discussed in Chapter 1 , to determine relative photoionization cross-sections using pulsed-field ionization zero-kinetic-energy photoelectron spectroscopy (PFI-ZEKE), it is essential that the efficiencies of Rydberg state stabilization at different ionization thresholds be similar. This appendix is directly based on a paper published by the author together with J. Hepburn and C. Alcaraz [102], which sought to address this problem, by comparing PFI-ZEKE signals observed at two ionization thresholds of argon, with the 
two partial photoionization cross-sections - which were known independently.

\section{B.2 Summary}

To examine issues concerning Rydberg-state stabilization in PFI-ZEKE spectroscopy, spectra at the first and second ionization thresholds of argon have been taken under different ion-density conditions. These provide a contrast between the signals from autoionizing and non-autoionizing Rydberg series. A careful comparison between the PFI-ZEKE spectra and relative partial photoionization cross-sections provides the first measurement of the efficiency of stabilization in PFI-ZEKE spectroscopy. This efficiency or quantum yield varies at the second ionization threshold with changing ion-density and principal quantum number, introducing ion-density dependent lineshape distortions. These changes may be ascribed to the competition between stabilization and autoionization of the Rydberg states and its variation with principal quantum number.

\section{B.3 Historical Background}

Pulsed-field ionization zero-kinetic-energy (PFI-ZEKE) photoelectron spectroscopy involves the selective detection of electrons field-ionized from optically excited high- $n$ Rydberg states ( $n=100$ to 300 ) [23, 146]. By scanning the excitation energy, one can map out the successive ionization thresholds of a neutral species, thereby determining the energy levels of the corresponding ion with significantly improved resolution compared to traditional photoelectron spectroscopy.

In addition to the new spectroscopic information, there has been considerable attention focused on obtaining information concerning the dynamics of the photoionization process from PFI-ZEKE. In particular, the higher resolution has opened up the possibility of comparing rotationally resolved PFI-ZEKE spectra with theoretical partial photoionization cross-sections in a number of interesting cases [147, 148]. For this comparison 
to be valid it is necessary that the detection efficiency of the optically excited Rydberg states be independent of the ionization threshold.

The essence of the PFI-ZEKE technique is the efficient discrimination against prompt photoelectrons from lower ionization thresholds. This is achieved by inserting a time delay between optical excitation and application of an ionizing electric-field pulse. This delay is typically on the order of $1 \mu \mathrm{s}$. To obtain any PFI-ZEKE signal, some fraction of the initially excited Rydberg states must survive this time delay. However, Chupka has pointed out that in certain cases [19], the experimental observation of a PFI-ZEKE signal is inconsistent with purely field-free, isolated atomic/molecular behavior. The unperturbed Rydberg states may have lifetimes much less than the waiting period between excitation and field ionization.

An illustrative example of this anomaly is the PFI-ZEKE spectra of argon [99], corresponding to the field ionization of Rydberg states with an electronically excited core $\left(\mathrm{Ar}^{+},{ }^{2} P_{1 / 2}\right)$. The Rydberg states were excited directly, by a single photon from the ground state of the neutral. At excitation energies between the ground state $\left({ }^{2} P_{3 / 2}\right)$ and the first excited state $\left({ }^{2} P_{1 / 2}\right)$ ionization thresholds, it is possible to collect a photo-ion yield spectrum which shows resonances corresponding to autoionization: excitation of a Rydberg state with a ${ }^{2} P_{1 / 2}$ ion core, which subsequently ionizes, ejecting an electron and leaving a ground state ion $\left({ }^{2} P_{3 / 2}\right)$. The oscillator strengths for transitions to these series suggest that they are also responsible for the excitation of the "ZEKE states" at the excited state threshold. Fitting the observed low- $n$ spectral widths to the well-known $n^{-3}$ scaling law allows one to extrapolate to the high-n typical of PFI-ZEKE. At $15 \mathrm{~cm}^{-1}$ below threshold ( $n=85$ ), a lifetime of $6 \mathrm{~ns}$ is predicted for the $s^{\prime}$ series (the longest lived optically bright series for excitation from the ground state $\left({ }^{1} S_{0}\right)$ of the neutral). This is inconsistent with the experimental observation of a field ionization signal at 200 ns. PFI-ZEKE spectra have also been taken at the $\mathrm{Kr}^{+},{ }^{2} P_{1 / 2}$ ionization threshold, after much longer time delays $[149,62]$. In the molecular case, Merkt et al. [150] have given a clear exposition of the necessity for a lifetime lengthening mechanism for PFI-ZEKE 
spectra of diatomic nitrogen. In this case predissociation and rotational autoionization are responsible for the decay of the high- $n$ Rydberg states. The discrepancy between delay time and extrapolated Rydberg-state lifetimes is common, and has been observed for both predissociating and autoionizing Rydberg states of both large and small molecules. It is clear that there must be some mechanism which stabilizes the initially excited low- $\ell$ Rydberg states, such that they survive until field ionization. In particular, the influence of perturbations external to the isolated atom/molecule must be considered.

For high- $n$ Rydberg states in the absence of external perturbations, the orbital angular momentum of the Rydberg electron $(\ell)$ is a reasonably good quantum number. Only low$\ell$ Rydberg series are optically bright in typical excitation schemes. While these low- $\ell$ Rydberg states can decay rapidly, the optically dark high- $\ell$ states decay slowly because of their reduced core penetration. The lifetimes of these states can be comparable with the PFI-ZEKE delay times. An electric field breaks the (almost) spherical symmetry of the electron, ion-core system, causing the Stark mixing of different $\ell$ 's. The stray electric fields typically present in the excitation region are sufficient to cause strong $\ell$-mixing of the high$n$ Rydberg states typical of PFI-ZEKE [19]. Since $\ell$ is no longer a good quantum number for the energy eigenstates, an initially prepared low- $\ell$ state can exhibit a time-dependent behavior, evolving from the initial excitation into a state with mixed- $\ell$ character. As the state gathers more high- $\ell$ character its autoionization (or predissociation) rate slows. Bixon and Jortner have done a time-independent calculation of the effect of $\ell$-mixing on Ar Rydberg states [151] Consideration of excitation through a "doorway" state allows them to derive the time-dependent decay of Rydberg states following excitation. These illustrate the rapid loss of Rydberg states due to autoionization immediately following excitation and then a much slower decay rate at longer times after the states are stabilized ( $\ell$-mixed). The relative yields of stabilized versus autoionized states depends on both $n$ and the strength of the mixing field.

While Stark mixing can lead to an increase in Rydberg state lifetimes, in some cases this is not sufficient to explain the presence of PFI-ZEKE signal. Bixon and Jortner 
[151] point out that their $\ell$-mixing calculations underestimate the experimental lifetimes observed by Merkt [99]. It has been suggested that the presence of nearby ions (from prompt ionization) combined with a small DC electric field can break the cylindrical symmetry of the system; ie., $m_{\ell}$, the projection of orbital angular momentum on an axis, is not a good quantum number of the energy eigenstates [19]. (Strictly speaking, it is the projection of the total angular momentum of the coupled electron, ion-core system, not $m_{\ell}$, which is the good quantum number of the homogeneous field energy eigenstates.) Since high- $\ell$ states have higher $m_{\ell}$ degeneracy than low- $\ell$ states, a statistical randomization of the $\ell, m_{\ell}$ quantum numbers lowers the decay rates more than $\ell$-mixing alone [19]. These mixing processes vary with $n$, so that external perturbations do not affect the low- $n$ lifetimes appreciably (where the $n^{3}$ scaling law is well obeyed).

Merkt concluded that both $\ell$ and $m_{\ell}$ mixing are required for observation of the $\mathrm{Ar}$ $\left({ }^{2} P_{1 / 2}\right.$ ionic core) PFI-ZEKE signal [99]. Merkt and Zare have calculated the ion-densities sufficient to cause the necessary cylindrical symmetry breaking [92]. In analogy with the purely $\ell$-mixing studied by Bixon and Jortner [151], one would expect short time competition between stabilization and autoionization, followed by a much slower decay of the $\ell$ and $m_{\ell}$ mixed states. The surrounding ion-density will alter the rate of stabilization and, hence, influence the relative yields of stabilized versus autoionized states. There are several experimental results which confirm that the extent of stabilization is influenced by the surrounding ion-density. Vrakking and Lee have observed the field ionization signal from Rydberg states of xenon converging on the ${ }^{2} P_{1 / 2}$ excited state core [152]. When they varied the number density of atoms and hence the ion-density (resulting from excitation leaving ${ }^{2} P_{3 / 2}$ ions), the field ionization signal increased with almost the square of the number density. This suggests the competition between autoionization and stabilization. In a set of femtosecond pump-probe experiments, the NRC (Ottawa) group has demonstrated the effects of ion-density on the PFI-ZEKE spectra of diatomic iodine $[153,154]$. By introducing background ions (prior to the femtosecond pump-probe pulses) with an independent laser, they were able to enhance Rydberg-state detection efficiency. 
Although the necessity of $\ell, m_{\ell}$ mixing has been established for certain specific cases, there remain several important questions, particularly in regards to practical application of the PFI-ZEKE technique. What fraction of initially prepared Rydberg electrons are detected? In other words, what is the "quantum yield"? How is this modified depending on external conditions (ion-density, DC-fields, etc.)? Does this fraction change at different ionization thresholds (where different Rydberg series are responsible)? If the quantum yield varies from threshold to threshold, this would severely hamper comparisons with theoretical cross-sections.

To examine the issues surrounding stabilization in PFI-ZEKE, argon has been studied at its first and second ionization thresholds, leaving ${ }^{2} P_{3 / 2}$ and ${ }^{2} P_{1 / 2}$ ionic cores respectively. The first two thresholds provide a nice contrast between PFI-ZEKE signals due to autoionizing ( ${ }^{2} P_{1 / 2}$ core) and non-autoionizing $\left({ }^{2} P_{3 / 2}\right.$ core) Rydberg states. Although Rydberg states approaching the ${ }^{2} P_{3 / 2}$ threshold can radiatively decay, this is negligible for the timescales and range of $n$ considered in this work $[19,155]$, allowing us to consider the Rydberg states approaching this threshold to be infinitely long-lived. If Rydberg-state stabilization is complete at the ${ }^{2} P_{1 / 2}$ threshold, the signals at the two thresholds should be proportional to the relative partial photoionization cross-sections at the thresholds. If stabilization is incomplete, the ${ }^{2} P_{1 / 2}$ signal would be expected to lose strength compared to the ${ }^{2} P_{3 / 2}$ signal.

In essence, comparing these two thresholds allows one to study PFI-ZEKE efficiency, while factoring out trivial aspects, such as electron detection and geometric collection efficiency, flight out of the detection region etc. These are the same at all ionization thresholds and can be corrected for by a simple multiplicative factor.

\section{B.4 Apparatus}

With the exception of minor changes, primarily to the vacuum system, the apparatus is as described by Kong et al. $\{62,61]$. The vacuum ultraviolet light (VUV) required 
for excitation was generated by four-wave sum-frequency mixing in a free jet of krypton $[70,68]$. Two standard pulsed nanosecond dye lasers were pumped with the second harmonic $(\mathrm{SH})$ and third harmonic $(\mathrm{TH})$ of a $\mathrm{Nd}$ :YAG laser with $\approx 10$ ns pulses and a $10 \mathrm{~Hz}$ repetition rate. The four-wave mixing process was resonantly enhanced by fixing the frequency-doubled output $\left(\nu_{1}\right)$ of the TH-pumped dye laser to a two-photon resonance in $\mathrm{Kr}\left(2 \nu_{1}=94093.7 \mathrm{~cm}^{-1}\right)$ [101]. The wavelength of the frequency-doubled SH-pumped dye laser $\left(\nu_{2}\right)$ was chosen to generate the desired sum-frequency $\left(2 \nu_{1}+\nu_{2}\right)$. The $\nu_{1}$ and $\nu_{2}$ beams were combined, then focused with a $30 \mathrm{~cm}$ lens into a pulsed jet of $\mathrm{Kr}$. The generated VUV was separated from the fundamentals $\left(\nu_{1}\right.$ and $\left.\nu_{2}\right)$ and undesired frequencies $\left(3 \nu_{1}, 2 \nu_{1}-\nu_{2}\right)$ and refocused by a one meter normal incidence monochromator (Acton Research Corporation, VM-521-X) into the main experimental region. The monochromator simply acts as a band-pass filter, allowing only the desired high-resolution $\left(\approx 1 \mathrm{~cm}^{-1}\right)$ VUV to pass. The VUV wavelength was calibrated by a combination of calibrating the two dye laser wavelengths using the optogalvanic effect in $\mathrm{Ne}$, and observing the known energy positions of Rydberg states of Ar between the first $\left({ }^{2} P_{3 / 2}\right)$ and second $\left({ }^{2} P_{1 / 2}\right)$ ionization thresholds. The system was windowless from generation of the VUV to the experimental region. Differential pumping of the mixing chamber, an intermediate buffer chamber, and the monochromator ensured that mixing gas loading on the main experimental chamber was light.

In the experimental chamber, the VUV intersected a free jet of argon at right angles. The Ar beam was emitted by pulsed valve (General Valve) with a $1 \mathrm{~mm}$ diameter nozzle located $5.2 \mathrm{~cm}$ away from the VUV beam axis. Unlike Ref.'s [62] and [61], the beam was unskimmed to allow higher beam densities to be studied. The vacuum chamber pressure was typically $10^{-7}$ torr and rose to $1 \times 10^{-5}$ torr with the $10 \mathrm{~Hz}$ pulsed argon beam on. The chamber was pumped with a $1000 \mathrm{~L} / \mathrm{s}$ turbo pump. Thus, the average gas load was $3.3 \times 10^{16}$ atoms per pulse. Assuming this gas was emitted in $500 \mu$ s long pulses, the average nozzle flow rate during a pulse was $6.6 \times 10^{19} \mathrm{~s}^{-1}$. The formulae of Beijerinck and Verster [91], as summarized by Miller [90] (his Eqn.'s 2.19a,b,c) may be used to compute 
the center-line particle flux at the appropriate distance $(5.2 \mathrm{~cm})$ from the nozzle opening. At this distance the argon atoms have essentially reached their terminal speed, computed to be $560 \mathrm{~m} / \mathrm{s}$ using Eqn. 2.2 of Miller [90]. The particle flux may be divided by this terminal speed to obtain a number density of $3 \times 10^{13} \mathrm{~cm}^{-3}$.

The Ar and VUV beam intersection region was centrally located between two gold mesh electrodes approximately $1.2 \mathrm{~cm}$ apart. The electrodes were used to apply a field ionizing pulse and to draw electrons into an electron spectrometer for the PFI-ZEKE experiments. ${ }^{1}$ These electrodes were also used to draw ions into a time of flight (TOF) spectrometer in the opposite direction from the electron spectrometer. Microchannel plate detectors are used for both ion and electron detection.

After passing through the Ar beam and the electron and ion spectrometers, the VUV reached a microchannel plate detector, used to monitor the VUV. All three microchannel plate detectors (for VUV, ions and electrons) consisted of two microchannel plates (Galileo Electro-Optics 1330-2500), sandwiched in series separated by $1.8 \mathrm{~mm}$, used in analog mode. Because of the importance of normalization in the experiments, all detectors were carefully checked for linearity.

After exiting the monochromator, the VUV could be attenuated by the Ar beam before it reaches the microchannel plate detector. In this case, ionization of the Ar would be primarily responsible for absorption. To estimate the size of the effect, the VUV was tuned over an autoionizing resonance $35 \mathrm{~cm}^{-1}$ above the first ionization threshold of Ar. Over a $5 \mathrm{~cm}^{-1}$ energy range the ionization cross-section jumps from roughly 50 to over $100 \mathrm{Mb}$ [156]. When scanning over this resonance, the reduction in VUV was less than $5 \%$ (this upper bound being limited by the signal to noise in the VUV detection, as no absorption was observed). This puts an upper bound of $1 \times 10^{-3} \mathrm{Mb}^{-1}$ on the $\mathrm{Ar}$ column density. Assuming a standard form for how beam density varies off-axis [90], this upper limit on column density is found to be consistent with the estimate of atomic beam

\footnotetext{
'This is the spectrometer described by Kong [62], not the spectrometer used in the TIPPS experiments described in this thesis.
} 
density given earlier. Since the total ionization cross-section in the energy ranges where PFI-ZEKE spectra were taken in this study is roughly $30 \mathrm{Mb}$ or less, the total attenuation of VUV must have been less than $3 \%$. Hence operation was under the so-called "thin sample" conditions, and the VUV fluence measured by the microchannel plate detector could be used for normalization.

Although the VUV microchannel plate detector was used throughout the experiment for monitoring and normalization, it was difficult to calibrate for absolute incident photon flux, particularly at the low voltages (and correspondingly low gains) used in the experiment. The typical microchannel plate signals together with a rough estimate of the detector gain suggested that a simple photodiode could be used to determine the VUV fluence per pulse (see, for example, Ref.'s [79] and [63]). By sliding a copper photodiode in front of the microchannel plate detector, an absolute calibration of the microchannel plate detector was made. It is concluded that the highest photon fluence in this work was roughly $4 \times 10^{7}$ photons per pulse.

Ion-density was an important factor in these experiments and must be known for both comparison with theory and other experiments. To determine ion-density, not only is the number of photons per pulse required but also their spatial distribution. Formulae for the spatial properties of the generated four-wave mixed signal as a function of properties of the fundamental beams are given by Lago [71]. After refocusing by the monochromator, astigmatism of the concave grating introduces a vertical spread estimated to be roughly $1 \mathrm{~mm}$ (see for example Ref. [63]). Defocusing over the $1 \mathrm{~cm}$ long ionization region and diffraction (due to the rulings) also degrade the spot size. Considering these effects, it is estimated that the majority of VUV was contained within a $1000 \mu \mathrm{m} \times 150 \mu \mathrm{m}$ patch throughout the distance traversed in the detection region. In principle, this could be confirmed by imaging the VUV spot; however this was not done. 


\section{B.5 Results and Discussion}

\section{B.5.1 Cross-section Measurements}

To compare PFI-ZEKE spectra at the two ionization thresholds, the relative photoionization cross-sections are desired. Before proceeding, it is necessary to establish what is meant by partial photoionization cross-sections at energies below the respective ionization threshold for the channel. At the first $\left({ }^{2} P_{3 / 2}\right)$ ionization threshold, a partial photoionization cross-section may be defined below the threshold. In this case, the defined cross-section is the same as the total photoabsorption cross-section convolved with the excitation bandwidth. This shows a smooth variation over the threshold. This is a consequence of both "continuity of oscillator strength" and the high number of Rydberg states contained within the excitation bandwidth at typical energies below threshold. Since ions cannot be created below the energetic threshold, the term "photoionization cross-section" is somewhat of a misnomer. Perhaps it would be better to refer to the "partial cross-section for excitation to the Rydberg pseudo-continuum". However, for brevity, we will use "photoionization cross-section".

The situation at the upper threshold is a bit trickier. In this case, $\sigma\left({ }^{2} P_{1 / 2}\right)$ below the ionization threshold is defined to be the fraction of the photoabsorption cross-section which results in excitation of high- $n$ Rydberg states approaching the second $\left({ }^{2} P_{1 / 2}\right)$ ionization threshold (convolved by the excitation bandwidth). Again, this will smoothly merge with the "proper" $\sigma\left({ }^{2} P_{1 / 2}\right)$ above threshold.

These definitions of cross-sections are independent of the convolving bandwidth, provided it is much greater than the Rydberg-state spacing at the energies of interest (conditions which are normally satisfied in PFI-ZEKE experiments).

It is noted that the extension of partial photoionization cross-sections to slightly below their energetic thresholds has been used by Softley and Hudson to compare multichannel quantum defect theory (MQDT) calculated photoionization cross-sections with 
PFI-ZEKE experiments [157]. The agreement between these calculations and previous experimental measurements is excellent.

As noted in the Apparatus section, the third harmonic of the resonance frequency is also generated in addition to the sum-frequency. By adjusting the monochromator, a quick change can be made between sending the sum-frequency or third harmonic into the experimental region. By recording light levels and $\mathrm{Ar}^{+}$ion-signals at these two wavelengths, the ratio of the total cross-section at the sum-frequency to that at the third harmonic can be determined. By multiplying the ratio of the relative cross-sections by the previously determined photoabsorption cross-section at the third harmonic wavelength [158], absolute cross-sections can be assigned to these measurements. From a different point of view, this is a convenient way of eliminating the need for normalization by molecular beam density, which while remaining fairly steady on a one-hour time-scale, can vary significantly from day to day.

This procedure has been applied to the measurement of photoionization cross-sections at both thresholds. At the second ionization threshold, the total photoionization crosssection is determined to be $31.0 \mathrm{Mb}$. This agrees well with a previous measurement of $31 \mathrm{Mb}$ [158]. Two channels contribute to the total ionization cross-section: $\sigma\left({ }^{2} P_{1 / 2}\right)$ and $\sigma\left({ }^{2} P_{3 / 2}\right)$. Samson et al. [100] have measured the branching ratio $\sigma\left({ }^{2} P_{3 / 2}\right) / \sigma\left({ }^{2} P_{1 / 2}\right)$ over $25 \mathrm{eV}$ starting at the second IP $\left({ }^{2} P_{1 / 2}\right)$. It changes surprisingly little from 1.93 over this energy range. Hence, it is assumed that this branching ratio may be applied at the ${ }^{2} P_{1 / 2}$ ionization threshold to obtain $\sigma\left({ }^{2} P_{1 / 2}\right)=10.6 \mathrm{Mb}$. This cross-section has a negligible variation over the range that the PFI-ZEKE spectra cover in this study. However, at the ${ }^{2} P_{3 / 2}$ ionization threshold $\sigma\left({ }^{2} P_{3 / 2}\right)$ varies strongly, due to the autoionization of Rydberg states converging on the ${ }^{2} P_{1 / 2}$ ionization threshold. In fact, two Rydberg states, $3 p^{5} 9 d^{\prime}\left(\frac{3}{2}\right)_{1}$ and $3 p^{5} 11 s^{\prime}\left(\frac{1}{2}\right)_{1}$, are located $21 \mathrm{~cm}^{-1}$ and $35 \mathrm{~cm}^{-1}$ above the ionization threshold, respectively [105]. Therefore, photoion yield spectra were taken as a function of photon energy in the vicinity of the first ionization threshold. The results are shown in Fig. B.1. 


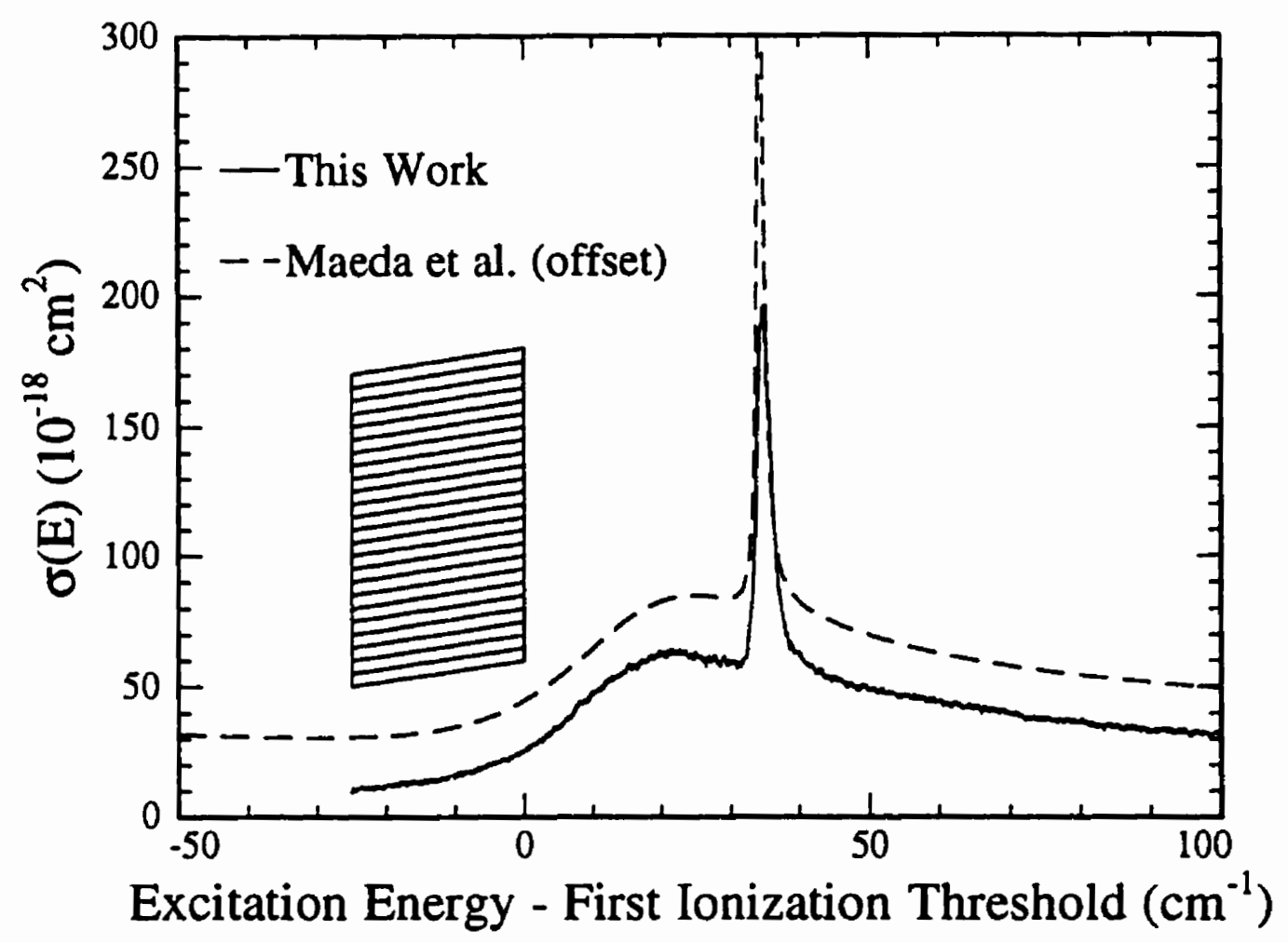

Figure B.1: Photoionization cross-section of argon near the first ionization threshold. The present results (solid line) are compared with the deconvolved photoabsorption measurements of Maeda et al., [156] which are offset vertically by $20 \mathrm{Mb}$ for clarity (1 Mb $=10^{-18} \mathrm{~cm}^{2}$ ). The shaded area represents the energy range for the PFI-ZEKE spectra at the first ionization threshold in this study. 
These determinations of cross-sections can be compared with previous studies. In Fig. B.1, the "deconvolved" photoabsorption spectrum due to Maeda et al. [156] has been overlaid. This was generated using the parameters and formulae contained in their paper. In the region where PFI-ZEKE spectra have been taken in this study (shaded in Fig. B.1), the cross-section is roughly $10 \%$ higher than that found by Maeda et al. [156]. However, this is consistent with the estimated uncertainties of both measurements. At the autoionization peak, the disagreement in cross-sections is larger, due in part to the finite excitation bandwidth.

\section{B.5.2 Quantum Yield}

PFI-ZEKE spectra were taken at the first two ionization thresholds of Ar with a pulsed field of $15 \mathrm{~V} / \mathrm{cm}$ delayed $1 \mu \mathrm{s}$ after excitation. These two spectra have been normalized by VUV and relative molecular beam density (determined by measurement of the third harmonic ionization signal). As discussed later, the ${ }^{2} P_{1 / 2}$ spectrum is specific to a certain set of VUV and molecular beam conditions. To compare the two PFI-ZEKE spectra with their respective partial photoionization cross-sections, they have been scaled by a common factor which matches the ${ }^{2} P_{3 / 2}$ spectrum to its cross-section over the range from $4 \mathrm{~cm}^{-1}$ to $12 \mathrm{~cm}^{-1}$ below threshold. The rescaled spectra and partial photoionization crosssections are shown in Fig. B.2, plotted as a function of energy below their two respective ionization thresholds at $127109.5 \mathrm{~cm}^{-1}$ and $128541.2 \mathrm{~cm}^{-1}$ [159].

An explanation for the dramatic differences in PFI-ZEKE lineshapes at the two ionization thresholds has been put forward by Merkt et al.[103]. The "shelf-like" structure in the ${ }^{2} P_{3 / 2}$ spectrum is due to incomplete field ionization at the red side of the peak. This structure was shown by Merkt et al. to scale in energy with varying ionizing field strength. For a certain range of energies below threshold the field ionization efficiency is expected to be $100 \%$. So as Fig. B.2 shows, within the range of $4 \mathrm{~cm}^{-1}$ to $12 \mathrm{~cm}^{-1}$ below threshold the ${ }^{2} P_{3 / 2}$ spectrum reflects the energy variation in cross-section. At the 


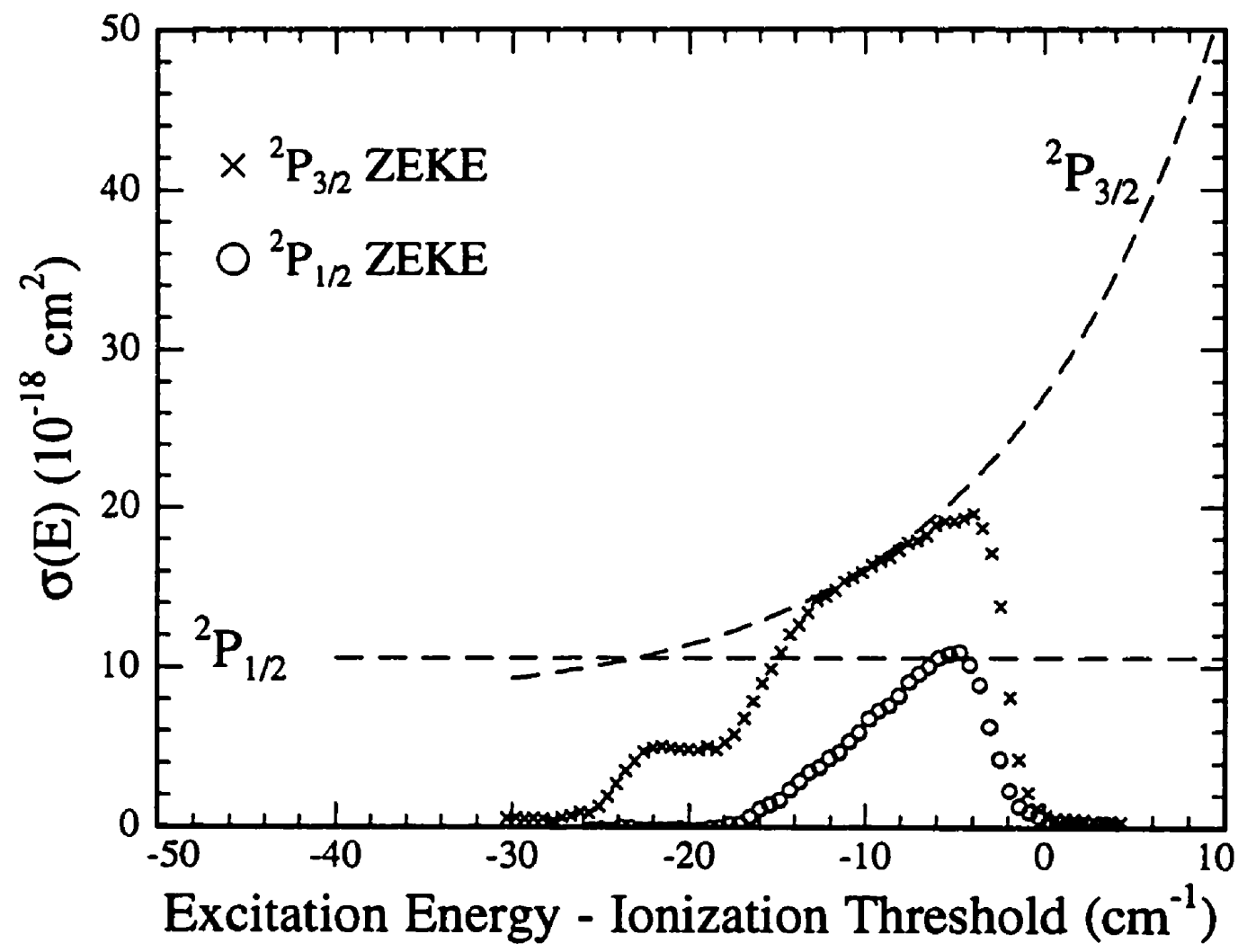

Figure B.2: Quantum yield measurements. A comparison between the photoionization cross-sections (dotted lines) and PFI-ZEKE spectra at the first $\left({ }^{2} P_{3 / 2}\right)$ and second $\left({ }^{2} P_{1 / 2}\right)$ ionization thresholds of argon is plotted as a function of excitation energy relative to the respective ionization thresholds. The two PFI-ZEKE spectra have been scaled vertically by a common factor to allow comparison with the ionization cross-sections. 
${ }^{2} P_{1 / 2}$ limit, it is hypothesized by Merkt et al. that the same low- $n$ states are "filtered" out in the waiting period by spin-orbit autoionization.

It is noted that the ${ }^{2} P_{1 / 2}$ PFI-ZEKE spectrum in Fig. B.2 does in fact approach the limit set by $\sigma\left({ }^{2} P_{1 / 2}\right)$. In other words, at one particular energy $\left(\approx 5 \mathrm{~cm}^{-1}\right.$ below threshold), the detection efficiency for excited Rydberg states is roughly the same as for the ${ }^{2} P_{3 / 2}$ non-autoionizing limit. There are several consequences of this observation.

There are two optically bright Rydberg series which could be responsible for initial excitation: the $s$ and $d$-series. Bixon and Jortner [151] have pointed out, based on a hydrogenic approximation, the $d$-series should have 20 times the oscillator strength ( $n$ independent) of the s-series. The actual photoionization yield spectrum between the two limits does seem to support this dramatic difference. As a conservative estimate, Merkt [99] used the field-free s-series lifetimes to show that the unperturbed Rydberg state lifetimes were too short to be observed. The present results show that the unperturbed lifetimes are in fact significantly smaller than this estimate because it is the optically bright, more quickly autoionizing $d$-series which must be responsible for the bulk of the PFI-ZEKE signal. If the $s$-series were responsible for observation, the ${ }^{2} P_{1 / 2}$ spectrum would be much weaker compared with the ${ }^{2} P_{3 / 2}$ spectrum.

Unfortunately, these quantum yield experiments do not have the requisite precision to establish whether or not the s-series is responsible for any of the PFI-ZEKE signal. The current experiment is not sensitive enough to see the small PFI-ZEKE signal losses that autoionization of the s-series would incur.

Despite reaching the maximum expected yield, the ${ }^{2} P_{1 / 2}$ PFI-ZEKE spectrum does show a dramatic variation with energy, which is not due to cross-section variation (the cross-section is flat over this scale). In examining the causes of this variation, it is important to consider two distinct possibilities: 1) The Rydberg states have decayed during the waiting period, and hence cannot be detected, or 2) while not decaying, a certain fraction of the Rydberg states are not detected in the electric-field ionization. The first type of 
explanation is contained in the "magic"-zone explanations of PFI-ZEKE lineshapes. At a certain critical energy below threshold, the Rydberg states are no longer sufficiently stabilized in the waiting period, and hence, cannot be detected after the waiting period. This places an upper limit on the width of the PFI-ZEKE line, independent of the strength of the ionizing field. The second possibility is illustrated in the work of Merkt et al. [103], who illustrate that for a certain range of $n$, it is possible to apply multiple successive field ionizing pulses, of the same magnitude and duration, and obtain multiple field ionization signals. In other words, the first and subsequent pulses do not ionize all of the optically prepared, stabilized states. Field ionization efficiency need not be $100 \%$. Any successful explanation of PFI-ZEKE lineshapes must consider both of these aspects determining the overall efficiency.

\section{B.5.3 Lineshape Variations with Ion-Density}

As mentioned, the ${ }^{2} P_{1 / 2}$ spectrum shown in Fig. B.2 was taken under a specific set of VUV and molecular beam conditions. To investigate the possible effects of ion-density on the quantum yield, the VUV fluence has been varied by changing the $\mathrm{Kr}$ mixing gas density. Measurement of the varying VUV microchannel plate readings should be a direct measurement of how the ion-density has changed in the experimental region. This is a distinct advantage of single-photon ionization. There are other possible approaches to varying the ion-density in PFI-ZEKE experiments $[152,154,160,161]$.

Figures B.3 and B.4 show the effect of varying the VUV, and hence, ion-density on the ${ }^{2} P_{1 / 2}$ PFI-ZEKE spectra. All of these were taken with a $15 \mathrm{~V} / \mathrm{cm}$ field-ionizing pulse, delayed $1 \mu \mathrm{s}$ after excitation, under approximately the same beam conditions, and were normalized by the VUV fluence. Based on the quantum yield measurements of the previous section, all spectra were scaled by a common factor, allowing one to interpret the vertical scale as referring to quantum yield. If the PFI-ZEKE spectra showed linear scaling with VUV, these normalized spectra should all be indistinguishable. This is 
indeed the case at the lower threshold $\left({ }^{2} P_{3 / 2}\right)$, where the scaling is perfect. The lower threshold verifies detection linearity and the soundness of this procedure. However, as Fig.'s B.3 and B.4 show, at the upper threshold, the lineshapes change dramatically, and the quantum yield drops from 1 with decreasing VUV fluence. The spectra have been divided into two figures, not only for clarity but also because they appear to show two separate phenomena at work.

At the higher ion-densities shown in Fig. B.3, increasing VUV fluence reduced the quantum yield for the high- $n$ portion of the line. Somehow the presence of ions inhibits the ability to see high- $n$ Rydberg states. As discussed in the previous section, experimentally no distinction can be made between destruction of these high- $n$ Rydberg states (by collisional ionization, for example) or a reduced field ionization efficiency. Because this effect appears at the high- $n$ edge of the spectrum, it is believed to be unrelated to autoionization. To test this, a PFI-ZEKE spectra of a mixed Ar, Kr beam was taken at the first ionization threshold of Ar (see Fig. B.5). krypton has a lower ionization threshold than argon, and hence provides a background source of ions. In this case, a virtually identical inhibition of the high-n signal is seen. Zhang et al. [161] have noted similar reductions in the high-n PFI-ZEKE signal, and have attributed these to ion-Rydberg or Rydberg-Rydberg interactions, as has Merkt [99].

At the lower ion-densities seen in Fig. B.4, the quantum yield improves at the highenergy side of the peak (it also appears to stop varying with ion-density). However, the peak quantum yield drops, and the loss at the low-energy edge is especially dramatic. This behavior is not observed in the ${ }^{2} P_{3 / 2}$ spectra with or without $\mathrm{Kr}$ present. In other words, the $n$-dependence of the quantum yield varies strongly for states which can autoionize. The fact that the presence of $\mathrm{Kr}$ does not effect the low-energy side of the ${ }^{2} P_{3 / 2}$ spectra appreciably at roughly $10 \mathrm{~cm}^{-1}$ below threshold provides strong evidence that possible changes in field-ionization efficiency with ion-density are not responsible for the variations in quantum yield (from $100 \%$ ) in the ${ }^{2} P_{1 / 2}$ spectra (see Fig. B.5). With no ion-density influence, it is expected that variations in field ionization efficiency would only effect the 


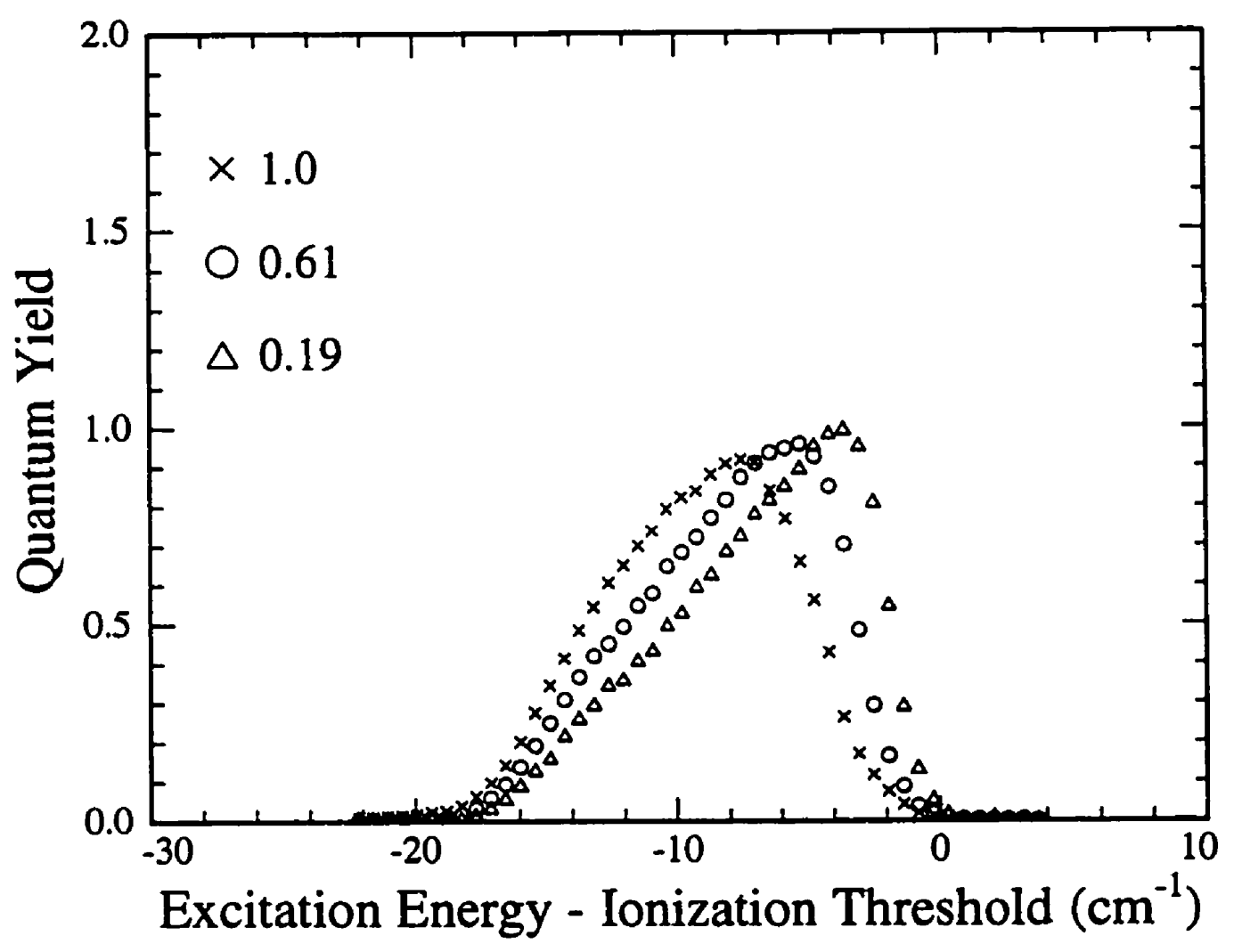

Figure B.3: Effects of ion-density on the PFI-ZEKE spectra of argon at the ${ }^{2} P_{1 / 2}$ limit: high ion densities. These three different PFI-ZEKE spectra were taken at different VUV fluences, and normalized by VUV fluence and molecular beam density. The products of VUV and molecular beam density for each of the three traces are contained in the legend (the units are arbitrary, but comparable with those in Fig. B.4). The maximum ion-density obtained (1.0 on the arbitrary scale) is approximately equal to $2 \times 10^{7} \mathrm{~cm}^{-3}$ (see text for details). Note the shifting of the high-energy edge to lower energies with higher ion-densities. 


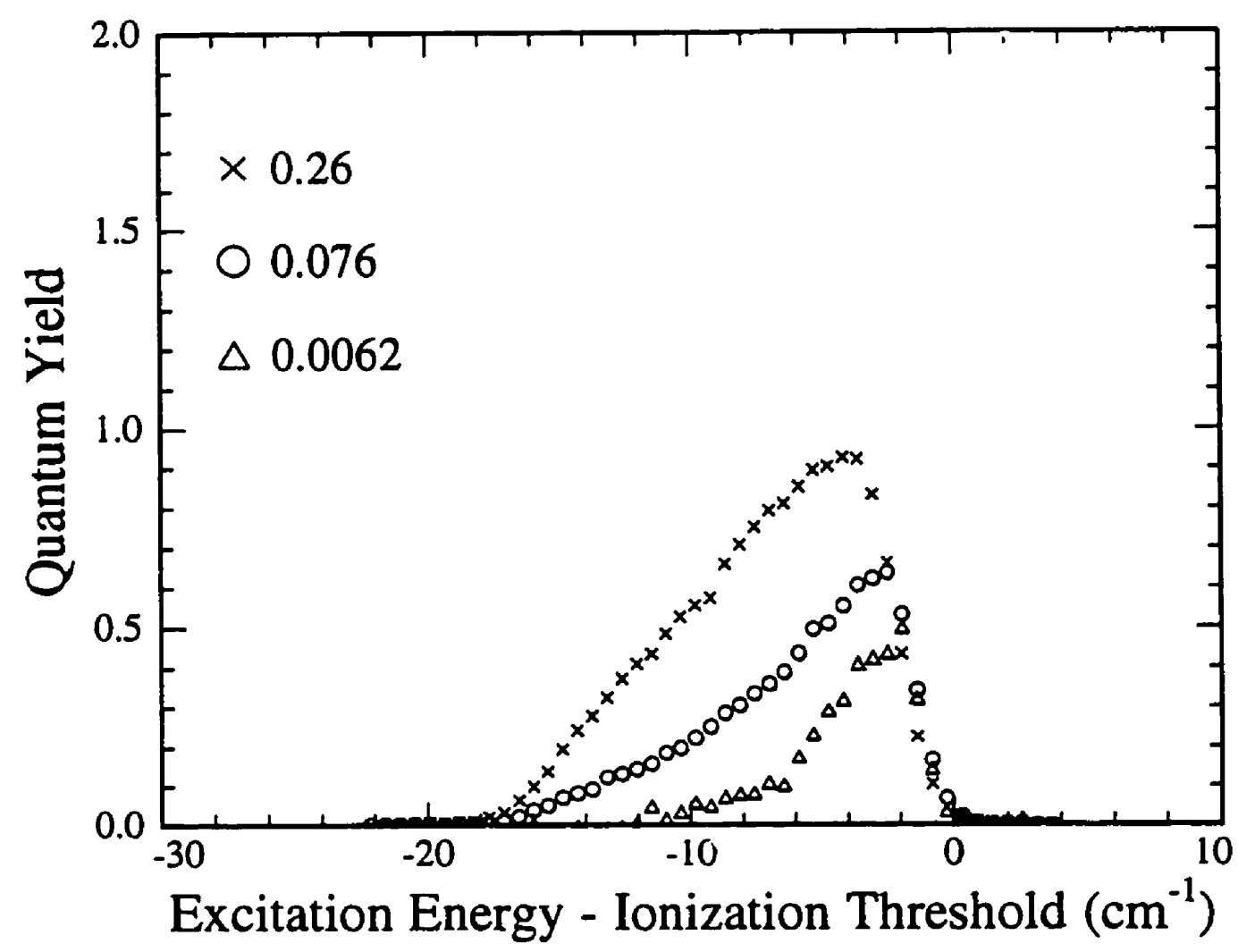

Figure B.4: Effects of ion-density on the PFI-ZEKE spectra of argon at the ${ }^{2} P_{1 / 2}$ limit: low ion densities. These three different PFI-ZEKE spectra were taken at different VUV fluences, and normalized by VUV fluence and molecular beam density. The products of VUV and molecular beam density for each of the three traces are contained in the legend (the units are arbitrary, but the same as those in Fig. B.3). Note that the high-energy edge is no longer shifting at these lower ion-densities. However the signal in the center of the peak drops dramatically with lower ion-densities. 


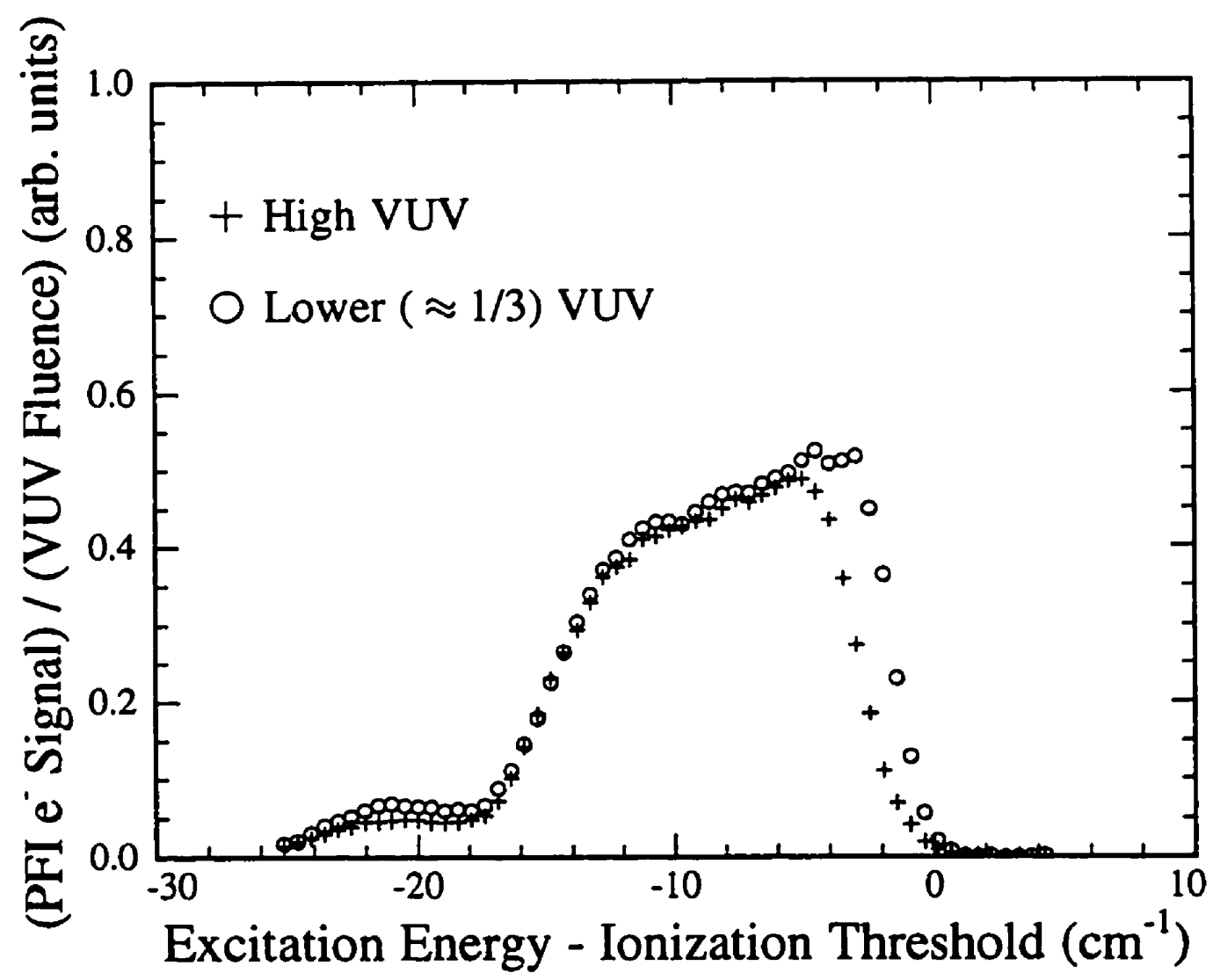

Figure B.5: Effects of ion-density on the PFI-ZEKE spectra of argon at the ${ }^{2} P_{3 / 2}$ limit. The beam contains a mixture of argon and krypton $(\mathrm{Kr}: \mathrm{Ar} \approx 2)$. Since $\mathrm{Kr}$ has a lower ionization potential than argon, it provides a source of ions at the first ionization threshold of $\operatorname{Ar}\left({ }^{2} P_{3 / 2}\right)$. The two spectra were taken under identical beam conditions with different VUV fluences, then normalized by VUV fluence. Note that the strength of the low-energy "shoulder" is less than in Fig. B.2. A different pulse generator was used for these spectra, and it is believed that the slew rate of the pulse effects the ionization yield in this region. 
${ }^{2} P_{1 / 2}$ spectra $18 \mathrm{~cm}^{-1}$ or further below threshold (in analogy with the behavior observed at the ${ }^{2} P_{3 / 2}$ threshold).

To enable comparison with other experimental conditions and theoretical calculations, the absolute ion-densities for the spectra in Fig.'s B.3 and B.4 are determined using the estimates for atomic beam density, VUV fluence, and spot size estimated in the experimental section. With a photoionization cross-section of $\sigma\left({ }^{2} P_{3 / 2}\right)=\sigma_{\text {tot }}-\sigma\left({ }^{2} P_{1 / 2}\right)=$ $20.4 \mathrm{Mb}$ at the ${ }^{2} P_{1 / 2}$ ionization threshold, the average ion-density across the spot at the end of the VUV pulse is computed to be $2 \times 10^{7} \mathrm{~cm}^{-3}$. These are the highest ion-density conditions in the experiments (1.0 Arbitrary units in Fig. B.3).

It is necessary to use caution when interpreting this ion-density estimate. It is clear that spatial variations of VUV and shot-to-shot fluctuations in both Ar beam intensity and VUV limit the characterization of ion-density with a single average quantity. There are also additional subtleties. For instance, the $\sigma\left({ }^{2} P_{3 / 2}\right)$ ionization cross-section used neglects the contribution from the autoionization of Rydberg states approaching the ${ }^{2} P_{1 / 2}$ limit (see the Cross-Section Measurements section). In fact, as discussed later, ion-density is believed to influence the fraction of autoionizing states, and hence create more ions. Another complication is that Rydberg states are formed and must be stabilized on time scales shorter than the length of the VUV pulse, thus experiencing a time-dependent ion-density which varies depending on when the Rydberg states are formed.

\section{B.5.4 Lifetime Measurements and a Lineshape Model}

To investigate the reduction in quantum yield at the upper $\left({ }^{2} P_{1 / 2}\right)$ ionization threshold with different ion-densities, an attempt to study the Rydberg states lifetimes was made. By varying the time delay from excitation to pulsed field extraction, and the corresponding detection gate, the decay curves as shown in Fig. B.6 were collected. The large "hump" in the first $0.1 \mu \mathrm{s}$, is contamination due to prompt electrons. This is determined by comparing these traces to ones taken at energies where no PFI-ZEKE signal is present 
after a $0.2 \mu$ s time delay (by moving to excitation energies several wavenumbers below the field ionization threshold). The decay on the $0.2 \mu \mathrm{s}$ to $1.0 \mu \mathrm{s}$ time scale was due to flight of the Rydberg atoms from beneath the detector during the waiting period. This was verified by observation of this identical decay at the ${ }^{2} P_{3 / 2}$ ionization threshold, and a decay rate which could be varied by changing the beam speed. Additionally the decay was independent of the energy below threshold (to $15 \mathrm{~cm}^{-1}$ below threshold, which was as far as this was tested). In other words, over the line, the decay was $n$-independent. The two traces shown in Fig. B.6 were taken under two different ion-density conditions, at $11 \mathrm{~cm}^{-1}$ below the ionization threshold, where the quantum yield dropped by $30 \%$ . Since the decay behavior is virtually identical on the $0.2 \mu \mathrm{s}$ to $1.0 \mu \mathrm{s}$ time scale, it is clear that if the reduction in quantum yield is due to Rydberg state loss, it must occur at short times ( $<200 \mathrm{~ns}$ ).

Based on these decay observations, a model for the quantum yield variations with ion-density can be formulated (under the assumption that the reduction in quantum yield is due to Rydberg state decay). Having established that both $\ell$ and $m_{\ell}$ mixing are necessary for stabilization, and recalling that $m_{\ell}$ mixing can be caused by surrounding ions, it seems reasonable that higher ion-densities result in greater stabilization yields. Since signal decay from 0.2 to $1.0 \mu$ s is observed to be independent of ion-density and $n$, the $n$-dependent loss of PFI-ZEKE signal must occur at short times. In fact, because at $11 \mathrm{~cm}^{-1}$ below threshold the $d$-series has a lifetime of $0.2 \mathrm{~ns}$, stabilization must occur on a comparable time-scale, if a PFI-ZEKE signal is to be observed. Discussion is simplified by the naive introduction of the "rate of stabilization". For sufficiently high ion-density, the stabilization rate could be greater than the autoionization rate, and hence all states which are excited are stabilized, and remain for field ionization. However, if the iondensity is reduced, the stabilization rate decreases, and autoionization could become competitive. At low- $n$, the autoionizaton rate is higher (because of the $n^{-3}$ scaling law), and less states can be stabilized before they autoionize. At higher- $n$, stabilization is more dominant over the slower autoionization rates. Of course, the stabilization rate also has 


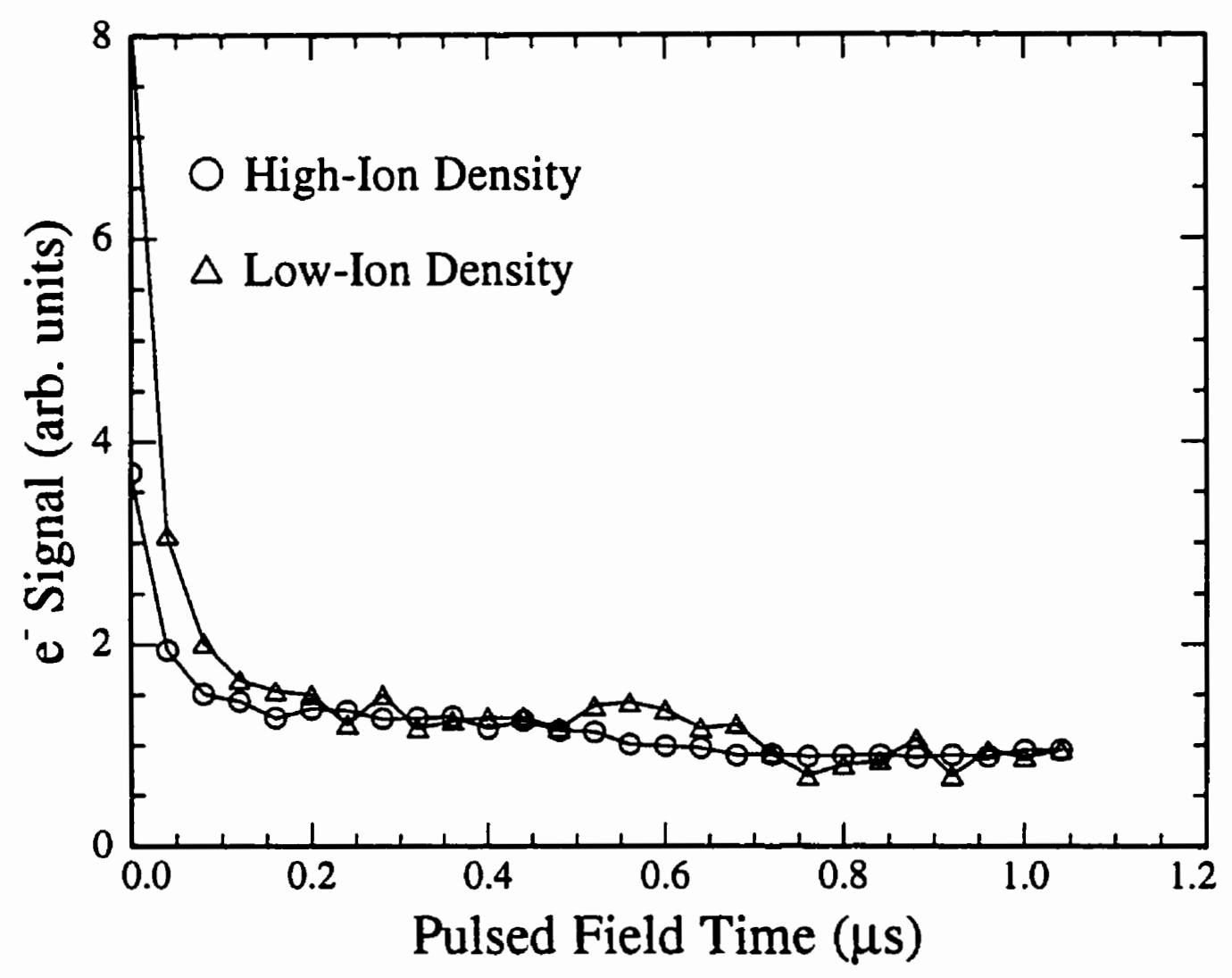

Figure B.6: PFI-ZEKE signal decay at $11 \mathrm{~cm}^{-1}$ below the ${ }^{2} P_{1 / 2}$ ionization threshold of argon. The time between excitation and application of the field ionization pulse is varied while simultaneously scanning the boxcar gate. These have been scaled relative to one another, for comparison of decay behaviour from 0.2 to $1 \mu \mathrm{s}$. 
an $n$ dependence. It is expected to increase with $n$, thus contributing to the asymmetry at low ion-densities. The $n$ dependence of both the stabilization and autoionization processes is a possible explanation of the lineshape distortion dominant in Fig. B.4 but also present in Fig. B.3 at low-n.

\section{B.6 Significance and Relationship to Other Work}

There appears to be some controversy concerning the role of background ions in PFIZEKE spectroscopy. This has been documented by Alt et al. [160], who observe that varying the background ion-density has no appreciable effect on the intensity or lifetime of their PFI-ZEKE signal. As they discuss, this is in contrast to the strong effect of ion-density found by Vrakking and Lee [152]. Despite this, it is important to note that no contradictory results have been found by different groups working on the same system, suggesting that the comprehensive explanation of the effects of ion-density will be subtie.

In fact, as Alt et al. [160] pointed out, varying ion-density can have effects on the PFIZEKE spectrum other than stabilization. Zavriyev et al. [162] mention a "false ZEKE" signal due to a space charge trapping effect. Slow electrons above threshold remain bound to the ion cloud, but are subsequently freed by field ionization, thereby contributing to a false PFI-ZEKE signal. The trapping of slow electrons has been discussed previously in conjunction with the suppression of low energy above threshold ionization (ATI) electrons [163]. This effect may be considered to be an extreme version of the $e^{-}$TOF distortions noticed by Meek et al. [164] in the early laser-based TOF photoelectron spectra. In general, we have found that the false PFI-ZEKE signal arising from trapping can be distinguished by observation of the dependence of the PFI-ZEKE signal on excitation energy (particularly if electrons can be observed above a known threshold).

While increasing ion-densities can introduce a false PFI-ZEKE signal through space charge trapping at the blue edge of the PFI-ZEKE lines, another phenomena competes to inhibit signal at the blue edge. As shown by Zhang et al. [161] and in Fig. B.3 of this 
work, increasing ion-density inhibits the observation of very high- $n$ Rydberg states. A quantitative explanation of the exact mechanism for this loss has not been made yet.

Another interesting ion-density effect in PFI-ZEKE is the charge exchange phenomena [165]. High-n Rydberg states have large cross-sections for charge exchange with ions. Smith and Chupka [166] have calculated the electron-transfer rates from Rydberg states to nearby ions under typical experimental conditions. Charge exchange offers an alternative stabilization mechanism for autoionizing Rydberg states. If an electron hops from an excited ionic core to a unexcited core, it will not be able to autoionize. This is clearly a stabilization mechanism which depends on ion-density. By collecting mass-analyzed threshold ionization (MATI) [29] spectra, Alt et al. [165] were able to observe a field ionization signal arising from one species of ion at the ionization thresholds of another species contained in their molecular beam, a signature of charge exchange. They were able to detect a small amount of $e^{-}$transfer or charge exchange (3\% of the parent ion signal), over a time period of a few $100 \mathrm{~ns}$ and at an ion-density of $5 \times 10^{6} \mathrm{~cm}^{-3}$. In the case of stabilization of Rydberg states approaching the ${ }^{2} P_{1 / 2}$ ionization threshold of Ar, charge exchange must occur over time periods comparable to the autoionization lifetimes, which are shorter than the time period allowed for exchange in Alt et al.'s experiment (where clearly charge exchange plays no role in stabilization). Experimentally it is difficult to distinguish between $\ell, m_{\ell}$-mixing and charge exchange stabilization in the presence of small DC-fields. They really represent two qualitatively different aspects of the same fundamental phenomena: perturbation of a Rydberg orbital by a nearby charge. However, considering the near $100 \%$ quantum yield observed at the high ion-densities in this work, it is felt that charge exchange is unlikely to explain the bulk of the stabilization effect in argon.

The extent or efficiency of stabilization is perhaps the key to understanding the apparently disparate results from different experimental groups. In the high ion-density regime of Fig. B.3, the actual peak height does not change dramatically with ion-density, and thus one might conclude that there is no ion-density effect, beyond the inhibition 
of high- $n$ signal as ion-density increases. However, when the efficiency of stabilization is small, varying the ion-density can have dramatic effects on the quantum yield, as observed in Fig. B.4 of this work, and by Vrakking and Lee [152]. In fact, recent work by Held et al. [167] on benzene has shown that for ion densities of less than $10^{5} \mathrm{~cm}^{-3}$, a slow decay of high- $n$ Rydberg states $(n \approx 200$ ) can be observed, while for higher ion-densities no decay can be seen. This possibly explains the earlier benzene results from the same group [160], where experiments were carried out under higher ion-density conditions and no ion-density effects were reported.

The concept of the efficiency of stabilization is important in the measurement of cross-sections. If stabilization is complete for all of the lines in a PFI-ZEKE spectrum, then their relative intensities should be accurate reflections of the corresponding partial photoionization cross-sections. If stabilization is incomplete, the different autoionization rates corresponding to Rydberg series converging on different ionization thresholds will distort the possible cross-section information available from PFI-ZEKE. Wales et al. [168] have hypothesized that the relative strengths of different rotational lines in their PFIZEKE spectra of $\mathrm{HCl}$ are influenced by variations in the autoionization rates, a nice manifestation of incomplete stabilization.

The different stabilization efficiency regimes were discussed by Vrakking et al. [154]. They noted that their spectra could be explained by assuming a stabilization efficiency proportional to ion-density, which is different from other workers who observe that their stabilization efficiency does not scale with ion-density. The present work appears to be the first demonstration of the transition between these two regimes (which involved varying the ion-density by more than two orders of magnitude).

There is one aspect of stabilization that the present work cannot address, namely the possibility of intramolecular processes which can occur for large molecules (ie. Benzene). Levine and co-workers (see for example, Ref. [169]), have considered the rotationalelectronic energy exchange between Rydberg electrons and ionic cores that may lead 
to stabilization. However, the external field effects discussed in this Appendix can be present in large molecular systems and, in fact, have been observed in ZEKE experiments on Benzene [167].

Initial work at the Chemical Dynamics Beamline at the Advanced Light Source (ALS) has demonstrated the applicability of PFI-ZEKE and MATI techniques with broadly tunable, narrow bandwidth VUV $[170,171]$. There is an exciting potential to apply this apparatus to a variety of interesting threshold photoionization cross-section measurements. However, because of the different repetition rate and intensity of the light source compared to laser based sources, one can expect to be working under significantly different ion-density conditions. In the MATI experiments on argon, no signal was seen at the ${ }^{2} P_{1 / 2}$ ionization threshold, despite the successful observation of the signal at the lower $\left({ }^{2} P_{3 / 2}\right)$ threshold, and both the first and second ionization thresholds of neon. We have approximated the field conditions for the ALS MATI experiments (a $0.34 \mathrm{~V} / \mathrm{cm}$ DC field present during photoexcitation), and find at the low ion-densities (typical of these experiments), the signal is too small to be detected, while increasing the ion-density dramatically improves the quantum yield. Over the same range of ion-densities with no DC-field, signal always remains detectable. This is consistent with the more recent PFI-ZEKE results from the same group [171], where under lower DC-field conditions a PFI-ZEKE signal was observed at the $A{ }^{2} P_{1 / 2}$ ionization threshold (with the same low ion-density conditions as the MATI experiments). Under the quite different experimental conditions of synchrotron apparatus and other future PFI-ZEKE applications, it is clear that an understanding of the important role of both ion-density and DC fields will prove critical in extracting useful cross-section information. 


\section{References}

[1] G. DiLonardo and A. E. Douglas, "The electronic spectrum of HF. I. The $B^{1} \Sigma^{+}-$ $X^{1} \Sigma^{+}$band system," Can. J. Phys., vol. 51, pp. 434-445, 1973.

[2] E. R. Cohen and B. N. Taylor, "The 1986 adjustment of the fundamental physical constants," Rev. Mod. Phys., vol. 59, pp. 1121-1148, 1987.

[3] H. Friedrich, Theoretical Atomic Physics, Springer-Verlag, Berlin, 1991.

[4] G. Herzberg, Molecular Spectra and Molecular Structure I. Spectra of Diatomic Molecules, Robert E. Krieger Publishing Co. Inc., Malabar, Florida, second edition, 1989.

[5] J. K. Cashion, "Testing of diatomic potential-energy functions by numerical methods," J. Chem. Phys., vol. 39, pp. 1872-1877, 1963.

[6] C. Asaro and A. Dalgarno, "Bound vibrational levels of the two lowest ${ }^{1} \Sigma^{+}$states of LiF, ${ }^{n}$ Chem. Phys. Lett., vol. 118, pp. 64-66, 1985.

[7] S. Pan and F. H. Mies, "Rydberg-like properties of rotational-vibrational levels and dissociation continuum associated with alkali-halide charge-transfer states," $J$. Chem. Phys., vol. 89, pp. 3096-3103, 1988.

[8] H. A. Bethe and E. E. Salpeter, Quantum Mechanics of One- and Two-Electron Atoms, Springer-Verlag, Berlin, 1957. 
[9] C. Fabre, M. Gross, J. M. Raimond, and S. Haroche, "Measuring atomic dimensions by transmission of Rydberg atoms through micrometer size slits," J. Phys. B: At. Mol. Opt. Phys., vol. 16, pp. L671-L677, 1983.

[10] T. F. Gallagher, Rydberg Atoms, Cambridge University Press, Cambridge, 1994.

[11] R. F. Stebbings and F. B. Dunning, Eds., Rydberg States of Atoms and Molecules, Cambridge University Press, London, 1983.

[12] F. Merkt, A. Osterwalder, R. Seiler, R. Signorell, H. Palm, H. Schmutz, and P. Gunzinger, "High Rydberg states of argon: Stark effect and field-ionization properties," J. Phys. B: At. Mol. Opt. Phys., vol. 31, pp. 1705-1724, 1998.

[13] W. E. Cooke and T. F. Gallagher, "Dependence of Rydberg-state field-ionization thresholds on $\left|m_{l}\right|, "$ Phys. Rev. A, vol. 17, pp. 1226-1228, 1978.

[14] S. T. Pratt, E. F. McCormack, J. L. Dehmer, and P. M. Dehmer, "Field-induced ion-pair formation in molecular hydrogen," Phys. Rev. Lett., vol. 68, pp. 584-587, 1992.

[15] J. R. Rubbmark, M. M. Kash, M. G. Littman, and D. Kleppner, "Dynamical effects at avoided level crossings: A study of the Landau-Zener effect using Rydberg atoms," Phys. Rev. A, vol. 23, pp. 3107-3117, 1981.

[16] D. Kleppner, M. G. Littman, and M. L. Zimmerman, "Rydberg atoms in strong fields," in Rydberg States of Atoms and Molecules, R. F. Stebbings and F. B. Dunning, Eds., chapter 3, pp. 73-116. Cambridge University Press, Cambridge, 1983.

[17] P. Pillet, H. B. van Linden van den Heuvell, W. W. Smith, R. Kachru, N. H. Tran, and T. F. Gallagher, "Microwave ionization of Rydberg atoms," Phys. Rev. A, vol. 30, pp. 280-294, 1984. 
[18] F. Merkt, "Molecules in high Rydberg states," Annu. Rev. Phys. Chem., vol. 48, pp. $675-709,1997$.

[19] W. A. Chupka, "Factors affecting lifetimes and resolution of Rydberg states observed in zero-electron-kinetic-energy spectroscopy," J. Chem. Phys., vol. 98, pp. 4520-4530, 1993.

[20] T. H. Jeys, G. W. Foltz, K. A. Smith, E. J. Beiting, F. G Kellert, F. B. Dunning, and R. F. Stebbings, "Diabatic field ionization of highly excited sodium atoms," Phys. Rev. Lett., vol. 44, pp. 390-393, 1980.

[21] J. D. D. Martin, "Pulsed field ionization zero kinetic energy electron spectroscopy of molecular hydrogen," M.S. thesis, University of Waterloo, 1995.

[22] K. Müller-Dethlefs, M. Sander, and E. W. Schlag, "Two-colour photoionization resonance spectroscopy of NO: Complete separation of rotation:l levels of $\mathrm{NO}^{+}$at the ionization threshold," Chem. Phys. Lett., vol. 112, pp. 291-294, 1984.

[23] G. Reiser, W. Habenicht, K. Müller-Dethlefs, and E. W. Schlag, "The ionization energy of nitric oxide," Chem. Phys. Lett., vol. 152, pp. 119-123, 1988.

[24] T. Baer and P.-M. Guyon, "A historical introduction to threshold photoionization," in High Resolution Laser Photoionizalion and Photoelectron Studies, 1. Powis, T. Baer, and C.-Y. Ng, Eds., chapter 1, pp. 1-20. John Wiley and Sons Ltd, West Sussex, England, 1995.

[25] L. Asbrink, "The photoelectron spectrum of $\mathrm{H}_{2}$, " Chem. Phys. Lett., vol. 7, pp. $549-552,1970$.

[26] J. E. Pollard, D. J. Trevor, J. E. Reutt, Y. T. Lee, and D. A. Shirley, "Rotationally resolved photoelectron spectroscopy of $\mathrm{n}-\mathrm{H}_{2}, \mathrm{p}-\mathrm{H}_{2}, \mathrm{HD}$, and $\mathrm{D}_{2}, " J$. Chem. Phys., vol. 77 , pp. 34-46, 1982. 
[27] K. Müller-Dethlefs and E. W. Schlag, "High-resolution zero kinetic energy (ZEKE) photoelectron spectroscopy of molecular systems," Annu. Rev. Phys. Chem., vol. 42, pp. $109-136,1991$.

[28] R. Lindner, K. Müller-Dethlefs, E. Wedum, K. Haber, and E. R. Grant, "On the shape of $\mathrm{C}_{6} \mathrm{H}_{6}^{+}$," Science, vol. 271, pp. 1698-1702, 1996.

[29] L. Zhu and P. Johnson, "Mass analyzed threshold ionization spectroscopy," J. Chem. Phys., vol. 94, pp. 5769-5771, 1991.

[30] R. N. Compton, "Negative-ion states," in Photophysics and Photochemistry in the Vacuum Ultraviolet, S. P. McGlynn, G. L. Findley, and R. H. Huebner, Eds. Dordrecht, Holland, 1985.

[31] W. A. Chupka and J. Berkowitz, "Kinetic energy of ions produced by photoionization of $\mathrm{HF}$ and $\mathrm{F}_{2},{ }^{n}$ J. Chem. Phys., vol. 54, pp. 5126-5132, 1971.

[32] M. S. De Vries, N. J. A. Van Veen, T. Baller, and A. E. De Vries, "Multiphoton ionization translational spectroscopy (MITS) of $\mathrm{I}_{2}$, " Chem. Phys., vol. 56, pp. $157-165,1981$.

[33] T. Munakata and T. Kasuya, "Ion-pair production from $\mathrm{CH}_{3} \mathrm{Cl}$ and $\mathrm{CH}_{3} \mathrm{Br}$ with $118 \mathrm{~nm}$ laser light, ${ }^{n}$ Chem. Phys. Lett., vol. 154, pp. 604-609, 1989.

[34] J. Berkowitz, "Photo-ion pair formation," in VUV and Soft X-Ray Photoionization, Uwe Becker and David A. Shirley, Eds., chapter 8. Plenum, New York, 1996.

[35] A. J. Yencha, D. Kaur, R. J. Donovan, A. Kvaran, A. Hopkirk, H. Lefebvre-Brion, and $\mathrm{F}$. Keller, "Ion-pair formation in the photodissociation of $\mathrm{HCl}$ and $\mathrm{DCl}, " J$. Chem. Phys., vol. 99, pp. 4986-4992, 1993.

[36] J. D. D. Martin and J. W. Hepburn, "Determination of bond dissociation energies by threshold ion-pair production spectroscopy: An improved $D_{0}(\mathrm{HCl}),{ }^{n} 1998$, Accepted for publication, J. Chem. Phys. 
[37] P. M. Dehmer and W. A. Chupka, "High resolution study of photoionization processes in $\mathrm{O}_{2}, " J$. Chem. Phys., vol. 62, pp. 4525-4534, 1975.

[38] H. Oertel, H. Schenk, and H. Baumgärtel, "Ion pair formation from photon irradiation of $\mathrm{O}_{2}$, $\mathrm{NO}$ and $\mathrm{CO}$ in $17-30 \mathrm{eV},{ }^{n}$ Chem. Phys., vol. 46, pp. 251-262, 1980.

[39] L. E. Berg, P. Erman, E. Källne, S. Sorensen, and G. Sundström, "Synchrotron radiation study of photoionization and photodissociation processes of $\mathrm{O}_{2}$ in the 12-26 eV region," Physica Scripta, vol. 44, pp. 328-335, 1991.

[40] K. Mitsuke, H. Yoshida, and H. Hattori, "Positive ion - negative ion coincidence spectroscopy of $\mathrm{O}_{2}$ and $\mathrm{H}_{2}$ using synchrotron radiation," Z. Phys. D, vol. 27, pp. $267-273,1993$.

[41] J. D. D. Martin and J. W. Hepburn, "Electric field induced dissociation of molecules in Rydberg-like highly vibrationally excited ion-pair states," Phys. Rev. Lett., vol. 79, pp. 3154-3157, 1997.

[42] J. Berkowitz, W. A. Chupka, P. M. Guyon, J. H. Holloway, and R. Spohr, "Photoionization mass spectrometric study of $F_{2}, \mathrm{HF}$, and DF, J. Chem. Phys., vol. 54, pp. 5165-5180, 1971.

[43] L. M. Tashiro, W. Ubachs, and R. N. Zare, "The HF and DF $B^{1} \Sigma^{+}-X^{1} \Sigma^{+}$ and $C^{1} \Pi-X^{1} \Sigma^{+}$band systems studied by $1 \mathrm{XUV}+1 \mathrm{UV}$ resonance enhanced multiphoton ionization," J. Mol. Spectry., vol. 138, pp. 89-101, 1989.

[44] W. A. Chupka, P. M. Dehmer, and W. T. Jivery, "High resolution photoionization study of ion-pair formation in $\mathrm{H}_{2}, \mathrm{HD}$, and $\mathrm{D}_{2}, " J$. Chem. Phys., vol. 63, pp. 3929-3944, 1975.

[45] A. H. Kung, R. H. Page, R. J. Larkin, Y. R. Shen, and Y. T. Lee, "Rydberg 
spectroscopy of $\mathrm{H}_{2}$ via stepwise resonant two-photon ion-pair $\mathrm{H}^{+}+\mathrm{H}^{-}$production," Phys. Rev. Lett., vol. 56, pp. 328-331, 1986.

[46] K. Mitsuke, S. Suzuki, T. Imamura, and I. Koyano, "Negative-ion mass spectrometric study of ion pair formation in the vacuum ultraviolet. I. $\mathrm{N}_{2} \mathrm{O} \rightarrow \mathrm{O}^{-}+\mathrm{N}_{2}^{+}, " J$. Chem. Phys., vol. 92, pp. 6556-6560, 1990.

[47] B. Ruscic and J. Berkowitz, "Photoion-pair formation and photoelectron-induced dissociative attachment in acetylene," J. Chem. Phys., vol. 93, pp. 5586-5593, 1990.

[48] M. Krauss, J. A. Walker, and V. H. Dibeler, "Mass spectrometric study of photoionization. X. Hydrogen Chloride and Methyl Halides," J. Res. Natl. Bur. Std., vol. 72A, pp. 281-293, 1968.

[49] S. Suzuki, K. Mitsuke, T. Imamura, and I. Koyano, "Negative-ion mass spectrometric study of ion-pair formation in the vacuum ultraviolet. VI. $\mathrm{CH}_{3} \mathrm{X} \rightarrow \mathrm{X}^{-}+\mathrm{CH}_{3}^{+}(\mathrm{X}=\mathrm{F}, \mathrm{Cl}, \mathrm{Br})$, J. Chem. Phys., vol. 96, pp. 7500-7505, 1992.

[50] E. P. Wigner, "On the behaviour of cross-sections near thresholds," Phys. Rev., vol. 73, pp. 1002-1009, 1948.

[51] E. E. Eyler, "Physics of near-threshold states in molecular hydrogen," in High Resolution Laser Photoionization and Photoelectron Studies, I. Powis, T. Baer, and C.-Y. $\mathrm{Ng}$, Eds., chapter 12, pp. 437-482. John Wiley and Sons Ltd, West Sussex, England, 1995.

[52] C. Blondel, "Recent experimental acheivements with negative ions," Physica Scripta., vol. T58, pp. 31-42, 1995.

[53] J. Berkowitz, Photoabsorption, Photoionization and Photoelectron Spectroscopy, Academic Press, Inc., New York, 1979. 
[54] A. L. Smith, "Continuity of differential oscillator strength through a dissociation limit; application to $\mathrm{O}_{2}$ Schumann-Runge and Herzberg I systems," J. Chem. Phys., vol. 55, pp. 4344-4350, 1971.

[55] P. M. Johnson and L. Zhu, "Mass analyzed threshold ionization: structural information for a mass spectrum and mass information for ionic spectroscopy," Int. J. Mass Spectrom. Ion Processes, vol. 131, pp. 193-209, 1994.

[56] J. Berkowitz, G. Barney Ellison, and D. Gutman, "Three methods to measure RH bond energies," J. Phys. Chem., vol. 98, pp. 2744-2765, 1994.

[57] A. G. Gaydon, Dissociation Energies and Spectra of Diatomic Molecules, Chapman and Hall, London, 1968.

[58] R. J. LeRoy and R. B. Bernstein, "Dissociation energies of diatomic molecules from vibrational spacings of higher levels: Application to the halogens," Chem. Phys. Lett., vol. 5, pp. 42-44, 1970.

[59] J. A. Blush, P. Chen, R. T. Wiedmann, and M. G. White, "Rotationally resolved threshold photoelectron spectrum of the methyl radical," J. Chem. Phys., vol. 98, pp. 3557-3559, 1993.

[60] R. Trainham, G. D. Fletcher, and D. J. Larson, "One- and two- photon detachment of the negative chloride ion," J. Phys. B: At. Mol. Opt. Phys., vol. 20, pp. L777L784, 1987.

[61] W. Kong, D. Rodgers, and J. W. Hepburn, "Pulsed field ionization threshold photoelectron spectroscopy with coherent vacuum ultraviolet: $\mathrm{NO}^{+}\left(a^{3} \Sigma^{+} v=\right.$ $0,1,2) \leftarrow \mathrm{NO}\left(X^{2} \Pi_{1 / 2}\right),{ }^{\prime}$ J. Chem. Phys., vol. 99, pp. 8571-8576, 1993.

[62] W. Kong, Photoionization Spectroscopy of Small Molecules Using Coherent Extreme Ultraviolet Radiation, Ph.D. thesis, University of Waterloo, 1993. 
[63] J. A. R. Samson, Techniques of Vacuum Ultraviolet Spectroscopy, Pied Publications, Lincoln, Nebraska, 1967.

[64] A. J. Yencha, A. Hopkirk, J. R. Grover, B-M. Cheng, H. Lefebvre-Brion, and F. Keller, "Ion-pair formation in the photodissociation of HF and DF," J. Chem. Phys., vol. 103, pp. 2882-2887, 1995.

[65] P. C. Hinnen, XUV-laser Spectroscopy of $\mathrm{H}_{2}$ and the Mystery of the Diffuse Interstellar Bands, Ph.D. thesis, Vrije Universiteit Amsterdam, 1997.

[66] J. W. Hepburn, "Coherent Vacuum Ultraviolet in Chemical Physics," Israel J. Chem., vol. 24, pp. 273-278, 1984.

[67] J. W. Hepburn, "Applications of coherent vacuum ultraviolet to photofragment and photoionization spectroscopy," in Vacuum Ultraviolet Photoionization and Photodissociation of Molecules and Clusters, C.Y. Ng, Ed., chapter 9. World Scientific Publishing Co. Pte. Ltd., P O Box 128, Farrer Road, Singapore 9128, 1991.

[68] J. W. Hepburn, "Generation of coherent vacuum ultraviolet radiation: Applications to high-resolution photoionization and photoelectron spectroscopy," in Laser Techniques in Chemistry, A. B. Myers and T. R. Rizzo, Eds., chapter 5, pp. 149-183. John Wiley and Sons, New York, 1995.

[69] D. C. Hanna, M. A. Yuratich, and D. Cotter, Non-Linear Optics of Free Atoms and Molecules, Springer-Verlag, Berlin, 1979.

[70] R. Hilbig, G. Hilber, A. Lago, B. Wolff, and R. Wallenstein, "Tunable coherent VUV radiation generated by nonlinear frequency conversion in gases," Comments At. Mol. Phys., vol. 18, pp. 157-180, 1986.

[71] A. Lago, Generation of Coherent Tunable Radiation in the Extreme Ultraviolet by Nonlinear Frequency Mixing of Pulsed Dye Laser Radiation in Rare Gases, Ph.D. thesis, Universität Bielefeld, 1987. 
[72] P. A. Franken, A. E. Hill, C. W. Peters, and G. Weinreich, "Generation of optical harmonics," Phys. Rev. Lett., vol. 7, pp. 118-119, 1961.

[73] R. T. Hodgson, P. P. Sorokin, and J. J. Wynne, "Tunable coherent vacuumultraviolet generation in atomic vapors," Phys. Rev. Lett., vol. 32, pp. 343-346, 1974.

[74] D. M. Bloom, J. T. Yardley, J. F. Young, and S. E. Harris, "Infrared up-conversion with resonantly two-photon pumped metal vapors," Appl. Phys. Lett., vol. 24, pp. 427-428, 1974.

[75] K. M. Leung, J. F. Ward, and B. J. Orr, "Two-photon resonant, optical thirdharmonic generation in cesium vapor," Phys. Rev. A, vol. 9, pp. 2440-2448, 1974.

[76] A. H. Kung, "Third harmonic generation in a pulsed supersonic jet of xenon," Opt. Lett., vol. 8, pp. 24, 1983.

[77] J. Bokor, P. H. Bucksbaum, and R. R. Freeman, "Generation of $35.5 \mathrm{~nm}$ coherent radiation," Opt. Lett., vol. 8, pp. 217, 1983.

[78] K. Kato, "Second-harmonic generation to $2048 \dot{\mathrm{A}}$ in $\beta-\mathrm{BaB}_{2} \mathrm{O}_{4}$," IEEE J. Quantum Electron., vol. QE-22, pp. 1013-1014, 1986.

[79] R. G. Tonkyn and M. G. White, "Compact vacuum ultraviolet source for photoelectron spectroscopy," Rev. Sci. Instrum., vol. 60, pp. 1245-1251, 1989.

[80] A. Lago, G. Hilber, and R. Wallenstein, "Optical-frequency conversion in gaseous media," Phys. Rev. A, vol. 36, pp. 3827-3836, 1987.

[81] "Spectra-Physics Lasers Inc.," 1330 Terra Bella Ave., P. O. Box 7013, Mountain View, CA, 94039-7013.

[82] A. E. Siegman, Lasers, University Science Books, Sausalito, CA, 1986. 
[83] "Lambda Physik," 3201 W. Commercial Blvd., Suite 110, Ft. Lauderdale, FL, 33309.

[84] R. Wallenstein and T. W. Hänsch, "Powerful dye laser oscillator-amplifier system for high-resolution spectroscopy," Opt. Commun., vol. 14, pp. 353-357, 1975.

[85] N. Melikechi, S. Gangopadhyay, and E. E. Eyler, "Phase dynamics in nanosecond pulsed dye laser amplification," J. Opt. Soc. Am. B, vol. 11, pp. 2402-2411, 1994.

[86] S. D. Bergeson, A. Balakrishnan, K. G. H. Baldwin, T. B. Lucatorto, J. P. Marangos, T. J. Mcllrath, T. R. O'Brian, S. L. Rolston, C. J. Sansonetti, Jesse Wen, N. Westbrook, C. H. Cheng, and E. E. Eyler, "Measurement of the He ground state Lamb shift via the two-photon $1^{1} \mathrm{~S}-2^{1} \mathrm{~S}$ transition," Phys. Rev. Lett., vol. 80 , pp. $3475-3478,1998$.

[87] V. G. Dmitriev, G. G. Gurzadyan, and D. N. Nikogosyan, Handbook of Nonlinear Optical Crystals, Springer-Verlag, Berlin, 2nd revised and updated edition, 1997.

[88] G. Scoles, Ed., Atomic and Molecular Beam Methods : Volume 1, Oxford University Press, New York, 1988.

[89] M. D. Morse, "Supersonic beam sources," in Methods of Experimental Physics: Atomic, Molecular and Optical Physics, Volume II, R. Hulet and F. B. Dunning, Eds. Academic Press, New York, 1996.

[90] D. R. Miller, "Free jet sources," in Atomic and Molecular Beam Methods: Volume 1, G. Scoles, Ed., chapter 2, pp. 14-53. Oxford University Press, New York, 1988.

[91] H. Beijerinck and N. Verster, "Absolute intensities and perpendicular temperatures of supersonic beams of polyatomic gases," Physica, vol. 111C, pp. 327-352, 1981.

[92] F. Merkt and R. N. Zare, "On the lifetimes of Rydberg states probed by delayed pulsed field ionization," J. Chem. Phys., vol. 101, pp. 3495-3505, 1994. 
[93] H.-J. Dietrich, R. Lindner, and K. Müller-Dethlefs, "A new ion detection scheme for ion spectroscopy equivalent to zero kinetic energy photoelectron spectroscopy," J. Chem. Phys., vol. 101, pp. 3399-3402, 1994.

[94] C. Jouvet, C. Delonder-Lardeux, S. Martrenchard-Barra, and D. Solgadi, "Massanalyzed threshold ionization (MATI) spectroscopy in a conventional time-of-hight mass spectrometer," Chem. Phys. Lett., vol. 198, pp. 419-423, 1992.

[95] W. C. Wiley and I. H. McLaren, "Time-of-flight mass spectrometer with improved resolution," Rev. Sci. Instrum., vol. 26, pp. 1150-1157, 1955.

[96] J. L. Wiza, "Microchannel plate detectors," Nucl. Instr. and Meth., vol. 162, pp. $587-601,1979$.

[97] L. Minnhagen, "Accurately measured and calculated ground-term combinations of Ar II," J. Opt. Soc. Am., vol. 61, pp. 1257-1262, 1971.

[98] L. Minnhagen, "Spectrum and the energy levels of neutral argon, Ar I," J. Opt. Soc. Am., vol. 63, pp. 1185-1198, 1973.

[99] F. Merkt, "Collisional and electric field effects in the delayed pulsed field ionization spectrum of argon," J. Chem. Phys., vol. 100, pp. 2623-2628, 1994.

[100] J. A. R. Samson, J. L. Gardner, and A. F. Starace, ${ }^{" 2} P_{3 / 2}:^{2} P_{1 / 2}$ partial photoionization cross-section ratios in the rare gases," Phys. Rev. A, vol. 12, pp. 1459-1463, 1975.

[101] C. E. Moore, Atomic Energy Levels, Vols. I, II and III (NSRDS-NBS35), U.S. Government Printing Office, Washington, D.C., 1971.

[102] J. D. D. Martin, J. W. Hepburn, and C. Alcaraz, "Efficiency of high-n Rydbergstate stabilization in pulsed-field ionization zero-kinetic-energy photoelectron spectroscopy," J. Phys. Chem. A, vol. 101, pp. 6728-6735, 1997. 
[103] F. Merkt, R. J. Rednall, S. R. Mackenzie, and T. P. Softley, "Electric field ionization of high Rydberg states of Ar with sequences of identical pulses," Phys. Rev. Lett., vol. 76, pp. 3526-3529, 1996.

[104] K. Yoshino and D. E. Freeman, "Absorption spectrum of xenon in the vacuum ultraviolet region," J. Opt. Soc. Am. B, vol. 2, pp. 1268-1274, 1985.

[105] K. Yoshino, "Absorption spectrum of the argon atom in the vacuum-ultraviolet region," J. Opt. Soc. Am., vol. 60, pp. 1220-1229, 1970.

[106] G. Herzberg and $\mathrm{Ch}$. Jungen, "Rydberg series and ionization potential of the $\mathrm{H}_{2}$ molecule," J. Mol. Spectry., vol. 41, pp. 425-486, 1972.

[107] K. P. Huber and G. Herzberg, Molecular Spectra and Molecular Structure IV. Constants of Diatomic Molecules, Van Nostrand Reinhold, New York, 1979.

[108] F. D. Rossini, "The heat of formation of hydrogen chloride and some related thermodynamic data," J. Res. Nat. Bur. Stand., vol. 9, pp. 679-702, 1932.

[109] H. Lefebvre-Brion and A. Suzor-Weiner, "Decay pathways for superexcited states of diatomic molecules obtained by photon absorption," Comments At. Mol. Phys., vol. 29, pp. 305-319, 1994.

[110] H. Lefebvre-Brion and R. W. Field, Perturbations in the Spectra of Diatomic Molecules, Academic Press, Orlando, 1986.

[111] W. R. Johnson and G. Soff, "The Lamb-shift in hydrogen-like atoms," At. Data Nucl. Data Tables, vol. 33, pp. 405-446, 1985.

[112] P. A. Rock, Chemical Thermodynamics, University Science Books, Mill Valley, CA, 1983.

[113] G. N. Lewis and M. Randall, Thermodynamics, McGraw-Hill, New York, 2nd edition, 1961. 
[114] M. W. Chase, Jr., C. A. Davies, J. R. Downey, Jr., D. J. Frurip, R. A. McDonald, and A. N. Syverud, JANAF Thermochemical Tables, AIP, New York, third edition, 1986.

[115] P. W. Atkins, Physical Chemistry, W. H. Freeman, New York, 4th edition, 1990.

[116] F. D. Rossini, Ed., Experimental Thermochemistry, Measurement of Heats of Reaction, Interscience, New York, 1956.

[117] Wallace L. Glab and Jan P. Hessler, "Efficient generation of $200 \mathrm{~nm}$ light in $\beta-$ $\mathrm{BaB}_{2} \mathrm{O}_{4}$," Appl. Opt., vol. 26, pp. 3181-3182, 1987.

[118] C. Amiot and J. Verges, "The magnetic dipole $a^{1} \Delta_{g} \rightarrow X^{3} \Sigma_{g}^{-}$transition in the oxygen afterglow," Can. J. Phys., vol. 59, pp. 1391-1398, 1981.

[119] P. F. Bernath, Spectra of Atoms and Molecules, Oxford, New York, 1995.

[120] P. C. Cosby and D. L. Huestis, "On the dissociation energy of $\mathrm{O}_{2}$ and the energy of the $\mathrm{O}_{2}^{+} b^{4} \Sigma_{g}^{+}$state," J. Chem. Phys., vol. 97, pp. 6108-6112, 1992.

[121] D. M. Neumark, K. R. Lykke, T. Andersen, and W. C. Lineberger, "Laser photodetachment measurement of the electron affinity of atomic oxygen," Phys. Rev. $A$, vol. 32, pp. 1890-1892, 1985.

[122] F. Merkt, S. R. Mackenzie, and T. P. Softley, "Preparation of ions in selected rotational states by delayed pulsed field ionization," J. Chem. Phys., vol. 99, pp. 4213-4214, 1993.

[123] S. R. Mackenzie and T. P. Softley, "New experimental method for studying rotationally state-selected ion-molecule reactions, ${ }^{n}$ J. Chem. Phys., vol. 101, pp. 10609-10617, 1994.

[124] O. Dutuit, C. Alcaraz, D. Gerlich, P. M. Guyon, J. Hepburn, C. MétayerZeitoun, J. B. Ozenne, M. Schweizer, and T. Weng, "A state-selected study 
of $\mathrm{Ar}^{+}\left({ }^{2} \mathrm{P}_{3 / 2,1 / 2}\right)+\mathrm{O}_{2}$ charge transfer at collision energies below $4 \mathrm{eV}$ using synchrotron radiation and guided beam techniques," Chem. Phys., vol. 209, pp. 177$194,1996$.

[125] J.-C. Brenot and M. Durup-Ferguson, "Multicoincidence detection in beam studies of ion-molecule reactions: Technique and application to $\mathrm{X}^{-}+\mathrm{H}_{2}$ reactions," in State-Selected and State-to-State Ion-Molecule Reaction Dynamics, C.-Y. Ng and M. Baer, Eds., chapter 5. Wiley, New York, 1992.

[126] W. T. Zemke, W. C. Stwalley, J. A. Coxon, and P. G. Hajigeorgiou, "Improved potential energy curves and dissociation energies for HF, DF and TF," Chem. Phys. Lett., vol. 177, pp. 412-418, 1991.

[127] V. H. Dibeler, J. A. Walker, and K. E. McCulloh, "Photoionization study of the dissociation energy of fluorine and the heat of formation of hydrogen fluoride," $J$. Chem. Phys., vol. 51, pp. 4230-4235, 1969.

[128] A. Mank, D. Rodgers, and J. W. Hepburn, "Threshold photoelectron spectroscopy of HF," Chem. Phys. Lett., vol. 219, pp. 169-173, 1994.

[129] J. Berkowitz, C. A. Mayhew, and B. Ruscic, "Photoion-pair formation in chlorine," Chem. Phys., vol. 123, pp. 317-328, 1988.

[130] C. Blondel, P. Cacciani, C. Delsart, and R. Trainham, "High-resolution determination of the electron affinity of fluorine and bromine using crossed ion and laser beams," Phys. Rev. A, vol. 40, pp. 3698-3701, 1989.

[131] J. A. Coxon and P. G. Hajigeorgiou, "Isotopic dependence of Born-Oppenheimer breakdown effects in diatomic hydrides: The $B^{1} \Sigma^{+}$and $X^{1} \Sigma^{+}$states of HF and DF," J. Mol. Spectry., vol. 142, pp. 254-278, 1990.

[132] J. Guo, A. Mank, and J. W. Hepburn, "Photoelectron angular distributions of 
rotationally selected NO Rydberg states," Phys. Rev. Lett., vol. 74, pp. 3584-3587, 1995.

[133] N. J. Dovichi, D. S. Moore, and R. A. Keller, "Use of the optogalvanic effect and the uranium atlas for wavelength calibration of pulsed lasers," Appl. Opt., vol. 21, pp. 1468-1473, 1982.

[134] A. M. Wilson, "Tunable pulsed dye laser calibration using the optogalvanic effect," Honours Fourth Year Physics Project Report, University of Waterloo, 1992.

[135] "SCP Science Catalog 1995/1996," 2367 Guenette, St. Laurent, QC, H4R 2E9.

[136] F. M. Phelps III, M.I.T. Wavelength Tables Vol. 2, Wavelengths by Elements, MIT Press, Cambridge, MA, 1982.

[137] A. R. Striganov and N. S. Sventitskii, Tables of Spectral Lines of Neutral and Ionized Atoms, Plenum, New York, 1968.

[138] D. J. Clouthier and J. Karolczak, "High-resoluton pulsed dye laser calibration in the 500-350 nm region using iodine atlas reference lines," Rev. Sci. Instrum., vol. 61, pp. 1607-1611, 1990.

[139] B. A. Palmer, R. A. Keller, and R. Engleman, "Atlas of Uranium Intensities in a Hollow Cathode Discharge, Report LA-8251-MS," 1980.

[140] B. A. Palmer, R. A. Keller, F. V. Kowalski, and J. L. Hall, "Accurate wave-number measurements of uranium spectral lines," J. Opt. Soc. Am., vol. 71, pp. 948-952, 1981.

[141] A. R. H. Cole, Tables of Wavenumbers for the Calibration of Infrared Spectrometers, Pergamon, Oxford, 1976.

[142] J. R. Taylor, An Introduction to Error Analysis, University Science Books, Mill Valley, CA, 1982. 
[143] K. D. Bonin, T. J. Mcllrath, and K. Yoshino, "High-resolution laser and classical spectroscopy of xenon autoionization," J. Opt. Soc. Am. B, vol. 2, pp. 1275-1283, 1985.

[144] K. S. E. Eikema, W. Ubachs, W. Vassen, and W. Hogervorst, "Lamb shift measurements in the $1^{1} S$ ground state of helium," Phys. Rev. A, vol. 55, pp. 1866-1884, 1997.

[145] N. Melikechi, S. Gangopadhyay, and E. E. Eyler, "Generation of vacuum ultraviolet radiation for precision laser spectroscopy," Appl. Opt., vol. 36, pp. 7776-7778, 1997.

[146] Ivan Powis, Tomas Baer, and Cheuk-Yiu Ng, Eds., High Resolution Laser Photoionization and Photoelectron Studies, John Wiley and Sons Ltd, West Sussex, England, 1995.

[147] K. Wang and V. McKoy, "Rotationally resolved photoelectron spectra at nearthreshold kinetic energies," in High Resolution Laser Photoionization and Photoelectron Studies, Ivan Powis, Tomas Baer, and Cheuk-Yiu Ng, Eds., chapter 8, Pp. 281-329. John Wiley and Sons Ltd, West Sussex, England, 1995.

[148] F. Merkt and T. P. Softley, "Rotational line intensities in zero kinetic energy photoelectron spectroscopy," Int. Rev. Phys. Chem., vol. 12, pp. 205-239, 1993.

[149] W. Kong, D. Rodgers, J. W. Hepburn, Kwangshi Wang, and V. McKoy, "Pulsedfield ionization threshold photoelectron spectroscopy with coherent extreme ultraviolet radiation: A comparision of $\mathrm{CO}$ and $\mathrm{N}_{2},{ }^{n}$ J. Chem. Phys., vol. 99, pp. 3159-3165, 1993.

[150] F. Merkt, S. R. Mackenzie, and T. P. Softley, "Rotational autoionization dynamics in high Rydberg states of nitrogen," J. Chem. Phys., vol. 103, pp. 4509-4518, 1995.

[151] M. Bixon and J. Jortner, "The dynamics of high autoionizing Rydberg states of Ar," J. Chem. Phys., vol. 103, pp. 4431-4446, 1995. 
[152] M. J. J. Vrakking and Y. T. Lee, "Lifetimes of Rydberg states in ZEKE experiments II.: Electric field induced and collisional enhancement of Xe autoionization lifetimes," J. Chem. Phys., vol. 102, pp. 8833-8841, 1995.

[153] I. Fischer, D. M. Villeneuve, M. J. J. Vrakking, and A. Stolow, "Femtosecond wavepacket dynamics studied by time-resolved photoelectron spectroscopy," J. Chem. Phys., vol. 102, pp. 5566-5569, 1995.

[154] M. J. J. Vrakking, I. Fischer, D. M. Villeneuve, and A. Stolow, "Collisional enhancement of Rydberg lifetimes observed in vibrational wave packet experiments," J. Chem. Phys., vol. 103, pp. 4538-4550, 1995.

[155] S. D. Chao, S. H. Lin, H. L. Selzle, and E. W. Schlag, "Comment of the spontaneous emission rates of ZEKE states," Chem. Phys. Lett., vol. 265, pp. 445-448, 1997.

[156] K. Maeda, K. Ueda, and K. Ito, "High-resolution measurements for photoabsorption cross-sections in the autoionization regions of $\mathrm{Ar}, \mathrm{Kr}$ and $\mathrm{Xe},{ }^{\prime}$ J. Phys. B: At. Mol. Opt. Phys., vol. 26, pp. 1541-1555, 1993.

[157] T. P. Softley and A. J. Hudson, "Multichannel quantum defect theory simulation of the zero-kinetic-energy photoelectron spectrum of $\mathrm{H}_{2}$, J. Chem. Phys., vol. 101, pp. 923-928, 1994.

[158] J. A. R. Samson, "Experimental photoionization cross-sections in argon from threshold to $280 \dot{A}, "$ J. Opt. Soc. Am., vol. 54, pp. 420-421, 1964.

[159] A. Muhlpfordt and U. Even, "Autoionizing Rydberg and zero kinetic energy states in Ar, J. Chem. Phys., vol. 103, pp. 4427-4430, 1995.

[160] C. Alt, W. G. Scherzer, H. L. Selzle, and E. W. Schlag, "Intensities and lifetimes of ZEKE spectra," Chem. Phys., vol. 240, pp. 457-460, 1995. 
[161] X. Zhang, J. M. Smith, and J. L. Knee, "Dynamics of high $n$ molecular Rydberg states with applications to mass analyzed ionization spectroscopy," J. Chem. Phys., vol. 99, pp. 3133-3136, 1993.

[162] A. Zavriyev, I. Fischer, D. M. Villeneuve, and A. Stolow, "Pondermotive effects in zero kinetic energy photoelectron spectroscopy with intense femtosecond pulses," Chem. Phys. Lett., vol. 234, pp. 281-288, 1995.

[163] M. Crance, "The influence of space charge on the angular distribution of electrons ejected after multiphoton ionisation," J. Phys. B: At. Mol. Opt. Phys., vol. 19, pp. L671-L676, 1986.

[164] J. T. Meek, S. R. Long, and J. P. Reilly, "Observation of polyatomic ion vibrational state distribution by laser photoelectron spectroscopy," J. Phys. Chem., vol. 86, pp. 2809-2811, 1982.

[165] C. Alt, W. G. Scherzer, H. L. Selzle, and E. W. Schlag, "Can molecular ZEKE states be generated by Rydberg electron pickup?," Chem. Phys. Letl., vol. 224, pp. $366-370,1994$.

[166] J. M. Smith and W. A. Chupka, "Electron transfer in high-n Rydberg states," J. Chem. Phys., vol. 103, pp. 3436-3439, 1995.

[167] A. Held, L. Y. Baranov, H. L. Selzle, and E. W. Schlag, "The interaction of a ZEKE electron with its ionic core: An experimental measurement," Chem. Phys. Lett., vol. 267, pp. 318-322, 1997.

[168] N. P. L. Wales, W. J. Buma, C. A. de Lange, and H. Lefebvre-Brion, "Dynamics of high- $n$ Rydberg states employed in zero kinetic enargy-pulsed field ionization spectroscopy via the $F^{1} \Delta_{2}, D^{1} \Pi_{1}$ and $f^{3} \Delta_{2}$ Rydberg states of $\mathrm{HCl},{ }^{n} J$. Chem. Phys., vol. 105, pp. 5702-5710, 1996. 
[169] E. Rabani, R. D. Levine, A. Mühlpfordt, and U. Even, "Dynamics and kinetics of molecular high Rydberg states in the presence of an electrical field: An experimental and classical computation study," J. Chem. Phys., vol. 102, pp. 1619-1638, 1995.

[170] C.-W. Hsu, K. T. Lu, M. Evans, Y. J. Chen, C. Y. Ng, and P. Heimann, "A high resolution photoionization study of $\mathrm{Ne}$ and $\mathrm{Ar}$ : Observation of mass analyzed threshold ions using synchrotron radiation and direct current electric fields," $J$. Chem. Phys., vol. 105, pp. 3950-3961, 1996.

[171] C. W. Hsu, M. Evans, C. Y. Ng, and P. Heimann, "High resolution threshold and pulsed field ionization photoelectron spectroscopy using multi-bunch synchrotron radiation," Rev. Sci. Instrum., vol. 68, pp. 1694-1702, 1997. 